\title{
MAGNETIC AND ELECTRONIC PROPERTIES OF IRON-BASED SUPERCONDUCTING SYSTEMS
}

\author{
BY \\ Sebastian Sambale
}

\begin{abstract}
A THESIS
SUbMitTed to The Victoria University of WeLLington

IN FULFILMENT OF THE REQUIREMENTS FOR THE DEGREE OF

Doctor of Philosophy
\end{abstract}

VICTORIA UNIVERSITY OF WELLINGTON

2015 


\section{Abstract}

This thesis is motivated by the large variety of high-temperature superconductors that contain iron in the superconducting layer. This number has grown rapidly since the discovery in 2008 of the iron-pnictides (and chalcogenides), where iron and arsenic form the superconducting layer. Also of interest are the iron-cuprate hybrid materials, where one out of three copper atoms is replaced by iron. The aim is to understand the superconducting, magnetic and electronic properties of these materials in respect to their iron content. This thesis describes some of these properties for the iron-pnictide compounds of $\mathrm{CeFeAsO}_{1-\mathrm{x}} \mathrm{F}_{\mathrm{x}}$ and $\mathrm{AFe}_{2} \mathrm{As}_{2}(\mathrm{~A}=\mathrm{Ba}, \mathrm{Sr})$, and for the ironcuprate hybrids of $\mathrm{FeSr}_{2} \mathrm{YCu}_{2} \mathrm{O}_{6+\mathrm{y}}$ and $\mathrm{FeSr}_{2} \mathrm{Y}_{2-\mathrm{x}} \mathrm{Ce}_{\mathrm{x}} \mathrm{Cu}_{2} \mathrm{O}_{10-\mathrm{y}}$.

Here it has been found that $\mathrm{CeFeAsO}_{1-\mathrm{x}} \mathrm{F}_{\mathrm{x}}$ follows a 3D fluctuation conductivity above the superconducting transition and the thermal activation energy is correlated to the critical current density within a two fluid-flux creep model below the superconducting transition. NMR measurements show that there is considerable charge disorder within the superconducting doping region. The $\mathrm{AFe}_{2} \mathrm{As}_{2}$ show a positive magnetoresistance, which could be interpreted through three-carrier transport. Superconducting samples of $\mathrm{SrFe}_{2} \mathrm{As}_{2}$ display a large enhancement in the magnetoresistance below the superconducting transition up to $1600 \%$, which is due to three-carrier transport through metallic and superconducting regions in an inhomogeneous state.

The superconducting properties of the iron-cuprate $\mathrm{FeSr}_{2} \mathrm{YCu}_{2} \mathrm{O}_{6+\mathrm{y}}$ in respect to the location of iron was studied under the influence of electron and hole doping and with additional magnetic impurities. $\mathrm{FeSr}_{2} \mathrm{Y}_{2-\mathrm{x}} \mathrm{Ce}_{\mathrm{x}} \mathrm{Cu}_{2} \mathrm{O}_{10-\mathrm{y}}$ shows a disorder induced spin-glass state and strong localization depending on the doping. 


\section{Acknowledgment}

Firstly, it is a great pleasure to express my special appreciations and thank my primary supervisor Dr. Grant V. M. Williams of Victoria University of Wellington and the MacDiarmid Institute for Advanced Materials and Nanotechnology. I would like to thank him for his constant encouragement and the support he gave from his vast knowledge and experience.

I am equally pleased to thank my secondary supervisor Dr. Shen V. Chong of Robinson Research Institute for all the helpful advices he has provided during my experiments and many helpful discussions. I would also like to thank him for introducting me into the fascinating sample preparation process of the iron-based superconductors.

The majority of this project was carried out at the Robinson Research Institute on the Callaghan Innovation Gracefield site, Lower Hutt. I would like to thank the many staff members that have happily helped in the characterization work, especially Dr. Martin Ryan (Callaghan Innovation, X-Ray diffraction), Dr. Ruth Knibbe and Sarah Spencer (Robertson Research Institute, scanning electron microscopy), Alison Speakman (Callaghan Innovation, library) and Dr. James Storey (Scientist) as well as the other members of the Robinson Research Institute for many helpful discussions. I would also like to thank the people from the Raman lab at Victoria University of Wellington for their help with Raman spectroscopy.

Special thanks to Prof. Jürgen Haase, Dr. Damian Rybicki and the rest of the MQF group of University Leipzig, Germany for their support during my NMR measurements at their group.

This work was funded by Marsden fund (VUW0917), the MacDiarmid Institute for Advanced Materials and Nanotechnology and the Ministry of 
Business, Innovation and Employment (C08X0705, C08X01206). The completion of this work was made possible thanks to a $\mathrm{PhD}$ Completion Scholarship from Victoria University Wellington.

I am also very grateful to my parents Stephan and Sabine as well as my sister Ariane and my brother Alexander for their support, love, guidance and encouragement. They made me the person that I am and without them nothing would have been possible. I would also like to thank all the other $\mathrm{PhD}$ students and staff members at Robinson Research Institute for all the enjoyable time we spent together outside of this project. 


\section{Contents}

\begin{tabular}{lll}
\hline & Introduction & 20
\end{tabular}

\begin{tabular}{lll}
\hline 2 & Theory & 26
\end{tabular}

2.1 Superconductivity . . . . . . . . . . . . . . . . . . . . 27

2.1 .1 Historical overview . . . . . . . . . . . . . . . 27

2.1 .2 The critical temperature . . . . . . . . . . . . . . . 29

$2.1 .3 \quad$ The critical magnetic field $\ldots \ldots \ldots$. . . . . . . . . . . 29

2.1 .4 The critical current density . . . . . . . . . . . . 31

2.2 Comparison between cuprates and iron-based superconductors 32

2.3 Magnetic order . . . . . . . . . . . . . . . . . 34

$2.3 .1 \quad$ Antiferromagnetic spin-density-wave order . . . . . . . 34

$2.3 .2 \quad$ Antiferromagnetic Mott insulator . . . . . . . . . . 36

2.3 .3 Spin Glasses . . . . . . . . . . . . . . . . . . 37

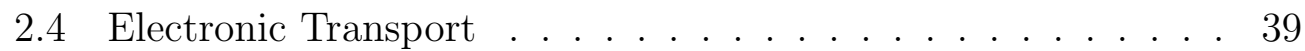

2.5 Magnetoresistance . . . . . . . . . . . . . . . . . . . 41

2.5.1 Quantum interference magnetoresistance . . . . . . . 43

2.5 .2 Three-carrier formalism . . . . . . . . . . . . 44

2.6 Nuclear Magnetic Resonance . . . . . . . . . . . . . . . . . . . 45

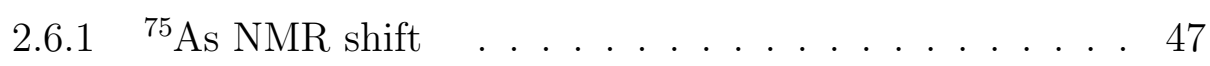

2.6 .2 The spin-lattice relaxation time, $T_{1}$ for ${ }^{75} \mathrm{As}$. . . . . 48

2.6 .3 The spin-spin relaxation time, $T_{2}$ for ${ }^{75} \mathrm{As}$. . . . . . 49

$\begin{array}{lll}3 & \text { Experimental details } & 50\end{array}$

3.1 Sample preparation . . . . . . . . . . . . . . . . . 51

3.1 .1 Cuprate superconductors . . . . . . . . . . . . . 51 
3.1 .2 Iron-based superconductor . . . . . . . . . . . . . 53

3.2 Sample characterization . . . . . . . . . . . . . . . . . 55

3.2 .1 X-ray diffraction . . . . . . . . . . . . . . 55

3.2 .2 Home built thermopower setup . . . . . . . . . . . 56

3.2 .3 Scanning electron microscope . . . . . . . . . . . . 57

3.2 .4 Raman spectroscopy . . . . . . . . . . . . . . . 58

3.3 The Physical Property Measurement Systems and the Magnetic Property Measurement System . . . . . . . . . . . . 59

3.3 .1 Resistivity and magnetoresistance . . . . . . . . . . . . 59

$\begin{array}{lll}3.3 .2 & \text { DC magnetization in a vibrating sample magnetometer } 61\end{array}$

3.3 .3 DC magnetization with the MPMS . . . . . . . . . 63

3.3 .4 AC magnetization . . . . . . . . . . . . . . . . . 63

3.4 Nuclear Magnetic Resonance setup . . . . . . . . . . . . . . . 64

3.4 .1 The resonant circuit . . . . . . . . . . . . . . 66

4 Magnetic and electronic properties of $\mathrm{CeFeAsO}_{1-\mathrm{x}} \mathrm{F}_{\mathrm{x}} \quad 68$

4.1 Introduction . . . . . . . . . . . . . . . . . . . . 68

4.2 Synthesis and structure . . . . . . . . . . . . . . . . . 70

4.3 Resistivity $\rho$. . . . . . . . . . . . . . . . . . . 72

$4.3 .1 \quad$ Zero field resistivity of the non-superconducting sample $\mathrm{x}=0.07 \ldots \ldots \ldots . \ldots . \ldots 72$

4.3 .2 Zero field resistance for superconducting samples . . . 73

$4.3 .3 \quad$ Fluctuation conductivity of superconducting samples . 74

4.3 .4 Field dependent resistivity in the superconducting state 76

4.4 Superconducting critical current density . . . . . . . . . . . . 82

4.5 Upper critical field, $B_{\mathrm{c} 2} \ldots \ldots \ldots \ldots$. . . . . . . . . . . 87

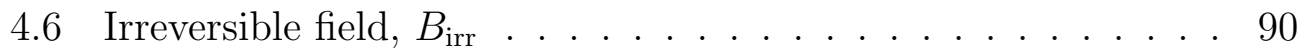

4.7 Susceptibility . . . . . . . . . . . . . . . . . . . . . . . 93

4.8 Summary and conclusions . . . . . . . . . . . . . . . . . . 96

$5 \quad{ }^{75} \mathrm{As} \mathrm{NMR}$ on $\mathrm{CeFeAsO}_{1-\mathrm{x}} \mathrm{F}_{\mathrm{x}}$ :

Spin and charge inhomogeneities 98

$5.1 \quad$ Introduction . . . . . . . . . . . . . . . . . . . . . . . . . . . . . . . . . . . . 98 
$5.2 \quad$ NMR on $\mathrm{CeFeAsO} \ldots \ldots \ldots$

5.2 .1 Temperature-dependence of ${ }^{75} \mathrm{As}$ NMR spectra . . . . . 99

5.2 .2 Temperature dependence of the spin-lattice relaxation in $\mathrm{CeFeAsO} \ldots \ldots \ldots$

5.2 .3 Temperature dependence of the spin-spin relaxation in $\mathrm{CeFeAsO} \ldots \ldots \ldots \ldots \ldots \ldots$

$5.3 \quad$ NMR of $\mathrm{CeFeAsO}_{0.93} \mathrm{~F}_{0.07} \ldots \ldots \ldots \ldots \ldots \ldots$

5.3 .1 NMR shift $\ldots \ldots \ldots \ldots 111$

5.3 .2 NMR linewidth . . . . . . . . . . . . . . . . . . . . . 112

5.3 .3 Temperature dependence of the spin-lattice relaxation of $\mathrm{CeFeAsO}_{0.93} \mathrm{~F}_{0.07} \ldots \ldots \ldots \ldots \ldots \ldots \ldots$

5.3 .4 Temperature dependence of the spin-spin relaxation in $\mathrm{CeFeAsO}_{0.93} \mathrm{~F}_{0.07} \ldots \ldots \ldots \ldots \ldots$

5.4 NMR on $\mathrm{CeFeAsO}_{0.87} \mathrm{~F}_{0.13} \ldots \ldots \ldots \ldots \ldots$

$5.4 .1 \quad$ Temperature dependence of ${ }^{75} \mathrm{As}$ NMR spectra. . . . . . 119

5.4 .2 Temperature dependence of the spin-lattice relaxation in $\mathrm{CeFeAsO}_{0.87} \mathrm{~F}_{0.13} \ldots \ldots \ldots 121$

5.4 .3 Temperature dependence of the spin-spin relaxation in $\mathrm{CeFeAsO}_{0.87} \mathrm{~F}_{0.13} \ldots \ldots \ldots \ldots . \ldots 122$

$5.5 \quad$ NMR on $\mathrm{CeFeAsO}_{0.80} \mathrm{~F}_{0.20} \ldots \ldots \ldots \ldots \ldots \ldots \ldots \ldots$

$5.5 .1 \quad$ Temperature dependence of the ${ }^{75} \mathrm{As}$ NMR spectra . . 124

5.5.2 Temperature dependence of the spin-lattice relaxation in $\mathrm{CeFeAsO}_{0.80} \mathrm{~F}_{0.20} \ldots \ldots \ldots \ldots \ldots \ldots \ldots \ldots \ldots$

5.5 .3 Temperature dependence of the spin-spin relaxation in $\mathrm{CeFeAsO}_{0.80} \mathrm{~F}_{0.20} \ldots \ldots \ldots \ldots 131$

$5.6 \quad$ Doping dependence of NMR properties . . . . . . . . . . . . 134

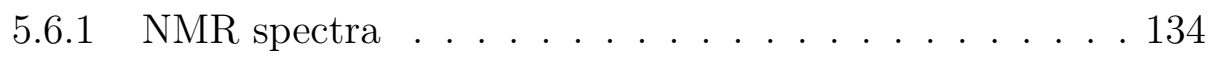

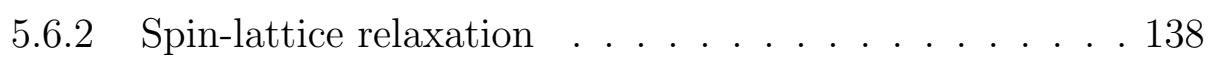

5.6 .3 Spin-spin relaxation. . . . . . . . . . . . . . . . . 140

5.7 Summary and conclusions . . . . . . . . . . . . . . . . 142

6 Magnetotransport study of $\mathrm{AFe}_{2} \mathrm{As}_{2}(\mathrm{~A}=\mathrm{Ba}, \mathrm{Sr}) \quad 144$

6.1 Introduction . . . . . . . . . . . . . . . . . 144 
6.2 Synthesis and structure . . . . . . . . . . . . . . . . . . 146

6.3 Resistivity . . . . . . . . . . . . . . . . . . . . . . . . . . . 148

6.4 Magnetoresistance . . . . . . . . . . . . . . . . . . . 150

6.4 .1 Magnetoresistance of $\mathrm{BaFe}_{2} \mathrm{As}_{2} \ldots \ldots \ldots \ldots$

6.4 .2 Magnetoresistance of $\mathrm{SrFe}_{2} \mathrm{As}_{2}$. . . . . . . . . . 153

6.4.3 Magnetoresistance of $\mathrm{SrFe}_{2} \mathrm{As}_{2}$ with 0.8 at\% $\mathrm{Ca}$

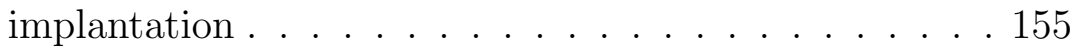

6.5 The aging effect on $\mathrm{SrFe}_{2} \mathrm{As}_{2} \ldots \ldots \ldots \ldots \ldots \ldots$

6.5 .1 Resistivity . . . . . . . . . . . . . . . . . . 158

$6.5 .2 \quad$ Magnetoresistance above $T_{\mathrm{C}} \ldots \ldots \ldots \ldots$

$6.5 .3 \quad$ Magnetoresistance below $T_{\mathrm{C}} \ldots \ldots \ldots \ldots$

6.5 .4 Reversibility . . . . . . . . . . . . . . . . . . 164

6.6 Summary and conclusions $\ldots \ldots \ldots \ldots$

7 The effect of electronic doping on the magnetic, electronic and vibrational properties of $\mathrm{FeSr}_{2} \mathrm{YCu}_{2} \mathrm{O}_{6+\mathrm{y}} \quad 168$

7.1 Introduction . . . . . . . . . . . . . . . . . . 168

7.2 Synthesis and structure . . . . . . . . . . . . . . . . . 170

7.3 Magnetic properties. . . . . . . . . . . . . . . . . . . . 172

7.4 Raman Spectroscopy . . . . . . . . . . . . . . . . . 178

7.5 The effects of Zn doping . . . . . . . . . . . . . . . 180

7.6 Summary and conclusions . . . . . . . . . . . . . 186

8 Magnetic and magnetotransport properties of

$\mathrm{FeSr}_{2} \mathrm{Y}_{2-\mathrm{x}} \mathrm{Ce}_{\mathrm{x}} \mathrm{Cu}_{2} \mathrm{O}_{10-\mathrm{y}}:$ Spin and electronic disorder $\quad 188$

8.1 Introduction . . . . . . . . . . . . . . . . 188

8.2 Synthesis and structure $\ldots \ldots \ldots$. . . . . . . . . . . . . . . . . 189

8.3 Magnetization . . . . . . . . . . . . . . . . . . . . . . 191

8.4 Susceptibility . . . . . . . . . . . . . . . . . . . . . . . . . . 194

8.5 Resistivity . . . . . . . . . . . . . . . . . . . . 196

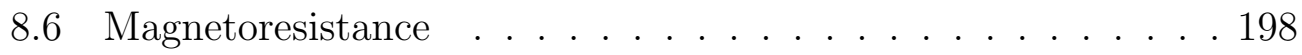

8.7 The effect of oxygen loading . . . . . . . . . . . . . 206

8.8 Summary and conclusions $\ldots \ldots \ldots \ldots$ 
$\begin{array}{lll}9 & \text { Summary } & 215\end{array}$

9.1 Iron-pnictide superconductor . . . . . . . . . . . . . . 216

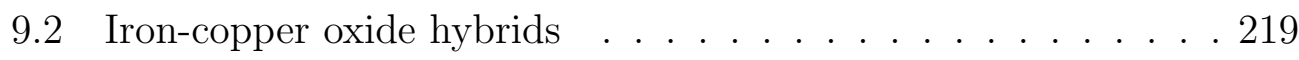

9.3 Outlook . . . . . . . . . . . . . . . . . . . . 221

\begin{tabular}{lr}
\hline Bibliography & 222
\end{tabular}

\begin{tabular}{|lr}
\hline A Appendix & 242
\end{tabular} 


\section{List of Figures}

2.1 Four different ways to determine $T_{\mathrm{C}}$. $\ldots \ldots \ldots$

2.2 The temperature dependence of the critical magnetic fields of type I and type II superconductors. . . . . . . . . . . . . . 30

2.3 The phase diagram of cuprates and iron-based superconductors. 33

2.4 Depiction of the co-linear antiferromagnetic order if the $\mathrm{Fe}$ atoms in the spin-density-wave state commonly observed in the 1111- and 122-family of the iron-based superconductors. . 34

$2.5 \quad$ NMR energy levels of ${ }^{75}$ As. . . . . . . . . . . . . . . . . 46

$3.1 \quad$ High temperature furnaces used for synthesis of the iron-cuprate hybrids. . . . . . . . . . . . . . . . . . . 52

3.2 Equipment used in the synthesis of iron-based superconductors. 53

3.3 An example of a double walled quartz tube sealed under reduced pressure with a $\mathrm{SrFe}_{2} \mathrm{As}_{2}$ sample surrounded by flux. . . 54

3.4 Philips PW1700 series powder diffractometer. . . . . . . . . 55

3.5 Room temperature thermopower measurement setup. . . . . . 56

3.6 Quanta SEM . . . . . . . . . . . . . . . . . 57

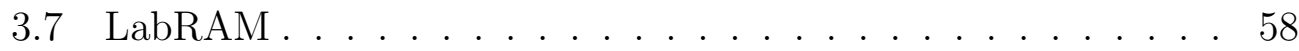

3.8 Magnetic lab at Robinson Research Institute (RRI) with a) PPMS and b) MPMS. . . . . . . . . . . . . . . . . . . 59

3.9 A sample of $\mathrm{BaFe}_{2} \mathrm{As}_{2}$ with copper wires connected for resistivity and magnetoresistance measurements. . . . . . . . . . . 60

3.10 Sample in the gelatin capsule in a brass sample holder for the VSM option of the PPMS. . . . . . . . . . . . . . . 61

3.11 NMR lab equipment. . . . . . . . . . . . . . . . 65 
3.12 NMR probehead with resonant circuit. . . . . . . . . . . 66

4.1 Crystal structure of $\mathrm{CeFeAsO}_{1-\mathrm{x}} \mathrm{F}_{\mathrm{x}} \ldots \ldots \ldots 71$

4.2 Temperature dependence of the resistivity for the 0.07 sample with a minimum around $150 \mathrm{~K}$. Inset: Enlarged low temperature region where there is a small decrease of the resistivity due to antiferromagnetic order of the Ce moments. . . . . . . 72

4.3 Temperature dependence of the resistance for all the superconducting samples with a Fermi liquid fit to the normal state resistance. . . . . . . . . . . . . . . . . . . 74

4.4 Fluctuation conductivity for all the superconducting samples with fitting to 3D regions. . . . . . . . . . . . . . 76

4.5 Temperature dependent resistance at around the superconducting transition at different applied fields. . . . . . . . . . 77

$4.6 \quad$ Example of Arrhenius plot for $\mathrm{x}=0.15$ at $2 \mathrm{~T}$ with a linear fit to the TAFF region. . . . . . . . . . . . . . . . 78

$4.7 \quad$ Arrhenius fits for Ce1111 for all superconducting samples. . . 79

4.8 Field dependence of $U_{0} / k_{\mathrm{B}}$ for all Ce1111 superconducting samples and fits to a power-law. Inset: Fitted exponent $\mathrm{n}$ of the power-law. . . . . . . . . . . . . . . 80

4.9 Magnetization field loop for $\mathrm{CeFeAsO}_{0.85} \mathrm{~F}_{0.15}$ at $5 \mathrm{~K}$. . . . . 82

4.10 Field dependence of the critical current density $J_{\mathrm{C}}$ for all four superconducting samples. . . . . . . . . . . . . . 83

4.11 Doping dependence of $B^{*}$ together with the exponent, $n$, from the power-law fit. . . . . . . . . . . . . . . 84

4.12 Doping dependence of $J_{\mathrm{C}}$ and the activation energy $U_{0} . \quad$. . 85

4.13 A linear relationship between $U_{0}$ and $J_{\mathrm{C}}$ within the two fluid flux creep model. . . . . . . . . . . . . . . . 86

4.14 Temperature dependence of the upper critical field, $B_{\mathrm{c} 2}$. . . . 87

4.15 Doping dependence of $d B_{\mathrm{c} 2} / d T$. . . . . . . . . . . . . . . 88

4.16 Limiting fields for $B_{\mathrm{c} 2}$ against doping. . . . . . . . . . . . . 88

4.17 Maki parameter, $\alpha$, against doping. . . . . . . . . . . . . . . 89

4.18 Irreversible magnetic field, $B_{\text {irr }}$. . . . . . . . . . . . . 90 
4.19 Plot of $\log \left(B_{\text {irr }}\right)$ vs $\log (1-t)$ for $\mathrm{CeFeAsO}_{1-\mathrm{x}} \mathrm{F}_{\mathrm{x}}$. Also plotted are fits to $(1-t)^{n}$ with $n \sim 1.7$ to 2.7 for $t<0.8$ (dashed lines) and $n>3$ for $t>0.8$ (solid lines). . . . . . . . . . . . 91

4.20 Scaling of $B_{\text {irr }}$ by $B_{\mathrm{c} 2}(0)$ and the fit to a power-law dependent behavior. . . . . . . . . . . . . . . 92

4.21 Temperature dependent susceptibility of $\mathrm{CeFeAsO}_{0.93} \mathrm{~F}_{0.07} \cdot$. . 93

4.22 Linear temperature dependent behavior of $1 /\left(\chi-\chi_{0}\right)$ for $\mathrm{CeFeAsO}_{0.93} \mathrm{~F}_{0.07} . \ldots \ldots \ldots \ldots . \ldots . \ldots . \ldots 94$

$5.1 \quad{ }^{75} \mathrm{As}$ NMR spectra of the central transition from CeFeAsO at room temperature. . . . . . . . . . . . . . . . . . . . . 99

$5.2 \quad{ }^{75}$ As NMR spectra of CeFeAsO at room temperature for $c \| B_{0}$ including satellite transitions. . . . . . . . . . . . 100

$5.3 \mathrm{CeFeAsO}{ }^{75} \mathrm{As}$ NMR spectra for both orientations at $170 \mathrm{~K}$ and room temperature. . . . . . . . . . . . . 100

5.4 CeFeAsO ${ }^{75} \mathrm{As}$ NMR Spectra for $c \| B_{0}$ below the SDW transition. . . . . . . . . . . . . . . . . 101

$5.5 \quad \mathrm{CeFeAsO}{ }^{75} \mathrm{As}$ NMR spectra for $a b \| B_{0}$ above and below the SDW transition. . . . . . . . . . . . . . . . . 101

5.6 Internal field, $B_{\text {int }}$, below the SDW transition. . . . . . . . . 102

5.7 Integrated intensities of the echo for different durations of $t$ at 295K in $c \| B_{0}$ where the fit to equation 2.19 is in red. . . . . 103

5.8 Temperature dependence of the spin-lattice relaxation time $T_{1}$ and rate, $1 / T_{1} \ldots \ldots \ldots \ldots$. . . . . . . . . . . . 104

5.9 Temperature dependence of the anisotropy ratio, $R_{1}$, of the spin lattice relaxation rates. . . . . . . . . . . . . . . . 105

5.10 Integrated intensity of the echo after different $t$ for $T_{2}$ at $295 \mathrm{~K}$

for $c \| B_{0}$. Also included is a fit to a Lorentz decay function
(equation 2.20). . . . . . . . . . . . . . 106

5.11 Temperature dependence of the spin-spin relaxation time, rate and anisotropy ratio for CeFeAsO in both magnetic field directions. . . . . . . . . . . . . . . . . . . 107 
5.12 Room temperature spectra of $\mathrm{CeFeAsO}_{0.93} \mathrm{~F}_{0.07}$ for both orientations including their satellite transitions. . . . . . . . . 108

5.13 Temperature dependence of the CT for temperatures from room temperature to $40 \mathrm{~K}$. . . . . . . . . . . . . . . . 109

5.14 Temperature dependence of the CT for temperatures $30 \mathrm{~K}$ to $10 \mathrm{~K}$ in both orientations. . . . . . . . . . . . . . 110

5.15 Spectra at $10 \mathrm{~K}$ for $c \| B_{0}$ with a fit to 2 sets of $3{ }^{75} \mathrm{As}$ NMR lines (green and blue) as well as a large Gaussian background (black). The cumulative fit is in red. . . . . . . . . . . . . . 110

5.16 Magnetic shift for both orientations with a fit to Curie-Weiss (CW) temperature dependent behavior. . . . . . . . . . . 111

5.17 Temperature dependence of the linewidth for both orientations. 113

5.18 Temperature dependence of the spin-lattice relaxation

in $\mathrm{CeFeAsO}_{0.93} \mathrm{~F}_{0.07}$. . . . . . . . . . . . . . . . . . . 113

5.19 Temperature dependence of the anisotropy ratio $R_{1}$ for $\mathrm{CeFeAsO}_{0.93} \mathrm{~F}_{0.07}$. . . . . . . . . . . . . . . 115

5.20 Temperature dependence of $T_{2}$ for both orientations and both components. . . . . . . . . . . . . . . 116

5.21 Fits to $T_{2}$ measurements with single or double Lorentzian components. . . . . . . . . . . . . . . 117

5.22 The temperature dependence of the spin-spin relaxation rate and the anisotropy ratio. . . . . . . . . . . . . . . . . . 118

5.23 Temperature dependence of the central transition for $\mathrm{x}=0.13$ for both magnetic field orientations. . . . . . . . . . . . . . 119

5.24 Temperature dependence of the NMR shift for both magnetic field orientations. . . . . . . . . . . . . . . . . 120

5.25 Temperature dependence of the spin-lattice relaxation for both magnetic field orientations. . . . . . . . . . . . . . . . . . 121

5.26 Temperature dependence of anisotropy ratio, $R_{1}$. . . . . . . 122

5.27 Temperature dependence of $T_{2}$ for both orientations and both components. . . . . . . . . . . . . . . . . . 122

5.28 Temperature dependence of the spin-spin relaxation rate and the anisotropy ratio for both magnetic field directions. . . . . 123 
5.29 Room temperature spectra for both orientations. . . . . . . . . 124

5.30 Temperature dependence of the central transition of $\mathrm{x}=0.20$ for both magnetic field directions. . . . . . . . . . . . . . 125

5.31 Temperature dependence of the magnetic shift in both magnetic field orientations and CW fits to the upper and lower frequency peaks. . . . . . . . . . . . . . 127

5.32 Temperature dependence of the NMR linewidth for both orientations. . . . . . . . . . . . . . . . . . . . . . 128

5.33 Temperature dependence of the spin-lattice relaxation in both magnetic field orientations. . . . . . . . . . . . . . 130

5.34 Double and single Lorentzian component fit of the spin-spin relaxation time for $c \| B_{0} . \ldots \ldots \ldots \ldots 132$

5.35 Temperature dependence of the spin-spin relaxation rate and the anisotropy ratio. . . . . . . . . . . . . . . . 133

5.36 The room temperature $(295 \mathrm{~K})$ spectra for all doping in $c \| B_{0}$ including their satellite transitions. . . . . . . . . . . . 134

5.37 (a) Doping dependence of quadrupole frequency and lattice parameter in $c \| B_{0}$ and their relative changes in (b). . . . . . 135

5.38 Temperature dependence of the NMR shift from the main line. 136

5.39 Temperature dependence of the linewidth. . . . . . . . . . . . 137

5.40 Temperature dependence of the spin-lattice relaxation for all

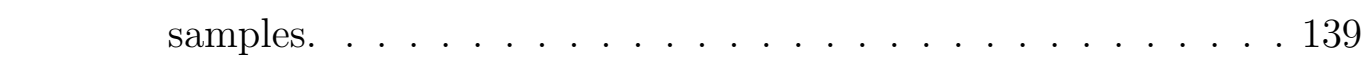

5.41 Temperature dependence of the anisotropy ratio $R_{1}$ for all doping. . . . . . . . . . . . . . . . 140

5.42 Temperature dependence of the spin-spin-relaxation rate and the anisotropy ratio $R_{2}$. . . . . . . . . . . . . . 141

$6.1 \quad$ Dirac cone energy dispersion. . . . . . . . . . . . . . 145

6.2 Crystal structure of $\mathrm{AFe}_{2} \mathrm{As}_{2}(\mathrm{~A}=\mathrm{Ba}, \mathrm{Sr}) . \quad \ldots . . . \quad \ldots 147$

6.3 Temperature-dependent resistivity of $\mathrm{BaFe}_{2} \mathrm{As}_{2}(\mathrm{Ba122}), \mathrm{SrFe}_{2} \mathrm{As}_{2}$

(Sr122), 0.8 at\%Ca implanted $\mathrm{SrFe}_{2} \mathrm{As}_{2}$ (Sr122 II) and the

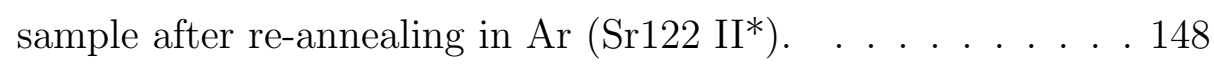

6.4 Magnetoresistance of $\mathrm{BaFe}_{2} \mathrm{As}_{2}$. . . . . . . . . . . . 150 
6.5 Derivative of the MR in the low-field region. . . . . . . . . . . 151

6.6 Temperature dependence of $B^{\star}$. . . . . . . . . . . . . . . . 152

6.7 The magnetoresistance of the non-superconducting $\mathrm{SrFe}_{2} \mathrm{As}_{2}$ crystal at different temperatures. . . . . . . . . . . . . 153

6.8 Derivative of the magnetoresistance for $\mathrm{SrFe}_{2} \mathrm{As}_{2}$ at different temperatures. . . . . . . . . . . . . . 154

6.9 MR for the non-superconducting $\mathrm{SrFe}_{2} \mathrm{As}_{2}$ crystal with 0.8 at\% Ca ions implanted at different temperatures. . . . . . . . . . 155

6.10 Derivative of the MR for $\mathrm{SrFe}_{2} \mathrm{As}_{2}$ with 0.8 at\% Ca implantation at different temperatures. . . . . . . . . . . . . 156

6.11 Time progressing of the temperature dependent resistivity of $\mathrm{SrFe}_{2} \mathrm{As}_{2} \ldots \ldots \ldots \ldots \ldots \ldots . \ldots \ldots$

6.12 Magnetoresistance of seven months old $\mathrm{SrFe}_{2} \mathrm{As}_{2}$. . . . . . . . 159

$6.13 B^{*}$ analysis for the seven months old $\mathrm{SrFe}_{2} \mathrm{As}_{2}$ crystal. . . . . 160

6.14 Temperature dependence of the (a) carrier mobilities and (b) the carrier densities obtained from the fit to Kim's threecarrier model. . . . . . . . . . . . . . . . . . . . . . . . . . 161

6.15 The magnetoresistance below the superconducting transition. 162

6.16 Magnetic field dependence of the MR for different temperatures for the re-annealed $\mathrm{SrFe}_{2} \mathrm{As}_{2}$ crystal. . . . . . . . . . 165

7.1 Crystal structure of $\mathrm{FeSr}_{2} \mathrm{YCu}_{2} \mathrm{O}_{6+\mathrm{y}}$ with $\mathrm{Fe}$ and $\mathrm{Cu}$ at their preferred position. . . . . . . . . . . . . 171

7.2 Temperature dependent magnetization for undoped Fe1212 at a magnetic field of $2 \mathrm{mT}$. . . . . . . . . . . . . . . . . 172

7.3 Doping dependence of the superconducting transition temperature and room temperature thermopower in $\mathrm{FeSr}_{2} \mathrm{YCu}_{2} \mathrm{O}_{6+\mathrm{y}}$. 173

7.4 Temperature dependence of the a) susceptibility and b) $1 / \chi-\chi_{0}$ for Fe1212. . . . . . . . . . . . . . . . . . . . 174

7.5 Doping dependence of the fit parameters. . . . . . . . . . . . 175

7.6 Doping dependence of the effective moment per formula unit. . 177

7.7 Room temperature Raman spectrum of an undoped Fe1212 sample. . . . . . . . . . . . . . . . . . . 178 
7.8 Doping dependence of the Raman peaks with electron and hole doping. . . . . . . . . . . . . . . . . . . . 179

7.9 The temperature dependence of ZFC and FC magnetization of $\mathrm{FeSr}_{2} \mathrm{YCu}_{2-\mathrm{x}} \mathrm{Zn}_{\mathrm{x}} \mathrm{O}_{6+\mathrm{y}}$ in an applied field of $2 \mathrm{mT}$. . . . . . . 181

$7.10 T_{\mathrm{C}}$ dependence on Zn doping . . . . . . . . . . . . . . . 182

$7.111 /\left(\chi-\chi_{0}\right)$ plot for Fe1212 samples together with the linear fit for a CW temperature dependence. . . . . . . . . . . . . . 183

7.12 Low field magnetization loops at 5K for Fe1212 with different

Z Zn content. . . . . . . . . . . . . . . . 185

8.1 Crystal structure of $\mathrm{FeSr}_{2} \mathrm{Y}_{2-\mathrm{x}} \mathrm{Ce}_{\mathrm{x}} \mathrm{Cu}_{2} \mathrm{O}_{10-\mathrm{y}} \cdot \ldots \ldots$. . . . . . 190

8.2 Temperature dependence of the ZFC and FC magnetization \begin{tabular}{|c|c|c|}
\hline for $\mathrm{FeSr}_{2} \mathrm{Y}_{1.3} \mathrm{Ce}_{0.7} \mathrm{Cu}_{2} \mathrm{O}_{10-\mathrm{y}}$ at an applied field of $2 \mathrm{mT}$. \\
\hline
\end{tabular}

8.3 Temperature dependence of the real part of the AC magnetization for seven different frequencies between $66 \mathrm{~Hz}$ to $10000 \mathrm{~Hz}$. 192

8.4 Relaxation times for different freezing temperatures with the fits to equation 2.6 . . . . . . . . . . . . . . . . . . 193

$8.5 \quad$ An example of the temperature dependent susceptibility for $\mathrm{x}=0.7$ (a) and the doping dependence of the CW temperature (b) and the effective moment (c). . . . . . . . . . . . . . . . 194

$8.6 \quad$ Resistivity against a temperature dependence of $T^{-1 / 3}(2 \mathrm{D})$ and $T^{-1 / 4}(3 \mathrm{D})$ for different Ce content. . . . . . . . . . . . 197

8.7 Magnetic field dependence of the MR for different Ce doping. 199

8.8 Temperature dependence of the MR at different magnetic fields and doping. . . . . . . . . . . . . . . . . . . . . 200

8.9 Magnetic field dependence of normalized MR for different temperatures going through the spin-glass transition. . . . . . . . 201

8.10 Magnetic field dependence of the MR at $5 \mathrm{~K}$ for parallel (black) and perpendicular (red) to the external magnetic field. . . . . 202

8.11 The MR for 10 and $20 \mathrm{~K}$ for the quadratic field dependence in the low field region. . . . . . . . . . . . . . . . . 204

8.12 The MR for 10 and $20 \mathrm{~K}$ for the linear field dependence in the high field region. . . . . . . . . . . . . . . 205 
8.13 Thermopower at room temperature for OR and OS samples of $\mathrm{Fe} 1222 . \quad \ldots \ldots \ldots \ldots$

8.14 ZFC and FC $(2 \mathrm{mT})$ magnetization for the oxygen reduced (OR) Fe1222 with 0.5 Ce doping. . . . . . . . . . . . . . . . 207

8.15 Temperature dependence of the susceptibility and $1 /\left(\chi-\chi_{0}\right)$ of the oxygen reduced (OR) and the oxygen saturated (OS) samples of Fe1222 with $\mathrm{x}=0.5$. . . . . . . . . . . . . . . . 208

8.16 Temperature dependence of the resistivity for OR and OS samples of $\mathrm{Fe} 1222$ with $\mathrm{x}=0.5 .7 \ldots$. . . . . . . . . . . 209

8.17 Plot of the resistivity against temperature for an oxygen saturated sample (solid curve). The dashed curve is a fit to equation 8.6. Also shown are the metallic (doted curve) and the $T^{-3 / 2}$ (dot-dash) contribution to the fit. . . . . . . . . . 211

A.1 XRD of $\mathrm{CeFeAsO}_{1-\mathrm{x}} \mathrm{F}_{\mathrm{x}}$, where the expected phase is identified and their crystal planes are shown in brackets. The arrows at lower angles show impurities, which are $1=\mathrm{FeAs}, 2=\mathrm{FeAs}_{2}$ and $3=\mathrm{Ce}_{3} \mathrm{Fe}_{4} \mathrm{O}_{3}$. The peaks at higher angles $\left(^{*}\right)$ are caused by the sample holder. . . . . . . . . . . . . . . . . . . . . . . . 243

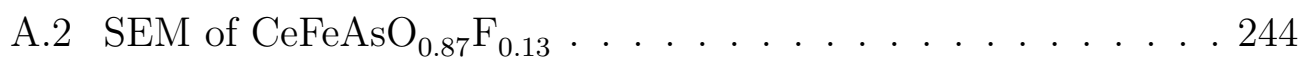

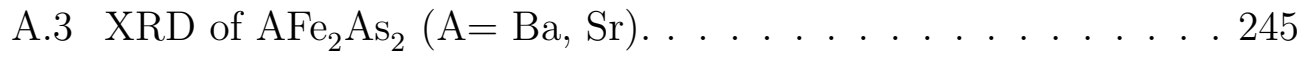

A.4 The measured pattern of pure $\mathrm{FeSr}_{2} \mathrm{YCu}_{2} \mathrm{O}_{6+\mathrm{y}}$ in black and the corresponding theoretical patterns of $\mathrm{FeSr}_{2} \mathrm{YCu}_{2} \mathrm{O}_{7.5}$ in green and $\mathrm{FeSr}_{2} \mathrm{YCu}_{2} \mathrm{O}_{6.8}$ in blue. . . . . . . . . . . . . . 246

A.5 The measured pattern of $\mathrm{FeSr}_{2} \mathrm{YCu}_{2} \mathrm{O}_{6+\mathrm{y}}$ with $0.1 \mathrm{Ca}$ in black and the corresponding theoretical patterns of $\mathrm{FeSr}_{2} \mathrm{YCu}_{2} \mathrm{O}_{7.5}$ in green and $\mathrm{FeSr}_{2} \mathrm{YCu}_{2} \mathrm{O}_{6.8}$ in blue. . . . . . . . . . . . . 247

A.6 The measured pattern of $\mathrm{FeSr}_{2} \mathrm{YCu}_{2} \mathrm{O}_{6+\mathrm{y}}$ with $0.2 \mathrm{Ca}$ in black and the corresponding theoretical patterns of $\mathrm{FeSr}_{2} \mathrm{YCu}_{2} \mathrm{O}_{7.5}$ in green and $\mathrm{FeSr}_{2} \mathrm{YCu}_{2} \mathrm{O}_{6.8}$ in blue. . . . . . . . . . . . . . . 248

A.7 The measured pattern of $\mathrm{FeSr}_{2} \mathrm{YCu}_{2} \mathrm{O}_{6+\mathrm{y}}$ with 0.1La in black and the corresponding theoretical patterns of $\mathrm{FeSr}_{2} \mathrm{YCu}_{2} \mathrm{O}_{7.5}$ in green and $\mathrm{FeSr}_{2} \mathrm{YCu}_{2} \mathrm{O}_{6.8}$ in blue. . . . . . . . . . . . . 249 
A.8 The measured pattern of $\mathrm{FeSr}_{2} \mathrm{YCu}_{2} \mathrm{O}_{6+\mathrm{y}}$ with 0.2La in black and the corresponding theoretical patterns of $\mathrm{FeSr}_{2} \mathrm{YCu}_{2} \mathrm{O}_{7.5}$ in green and $\mathrm{FeSr}_{2} \mathrm{YCu}_{2} \mathrm{O}_{6.8}$ in blue. . . . . . . . . . . . . 250

A.9 XRD pattern of Fe1222 with $0.3 \mathrm{Ce}$. . . . . . . . . . . . . . . 251

A.10 XRD pattern of Fe1222 with 0.5Ce . . . . . . . . . . . . . . . 252

A.11 XRD pattern of Fe1222 with 0.7Ce . . . . . . . . . . . . . 253

A.12 XRD pattern of Fe1222 with 0.9Ce . . . . . . . . . . . . . . 254 


\section{List of Tables}

4.1 Field dependence of $U_{0} / k_{\mathrm{B}}$ for all Ce1111 superconducting samples. . . . . . . . . . . . . . . . . 80

$4.2 \quad$ Fitted exponent values of the power-law dependence of the irreversible field $B_{\text {irr }}$. . . . . . . . . . . . . . . . . . . 91

$5.1 \quad$ Internal field, $B_{\text {int }}^{c}$, in the $c$-direction below the SDW transition. . . . . . . . . . . . . . . 102

$5.2 \quad T_{1}$ for CeFeAsO at different temperatures and both orientations and the experimental uncertainty. . . . . . . . . . . 103

$5.3 \quad T_{2}$ for CeFeAsO at different temperatures and both orientations and their experimental uncertainty. . . . . . . . . . 106

5.4 Obtained fit-parameters from a CW fit to the NMR shift data. 112

5.5 Obtained fit parameters from the CW fit to the linewidth. . . 113

$5.6 \quad$ Fitted parameters from a 2D spin fluctuations fit to the data in figure $\mid 5.18 \mathrm{~b}$. . . . . . . . . . . . . . . . . . . . . 114

5.7 Two components of $T_{2}$ for $\mathrm{CeFeAsO}_{0.93} \mathrm{~F}_{0.07}$ at different temperatures and orientations including their uncertainty (in brackets.$\ldots \ldots \ldots \ldots \ldots$

5.8 CW fit parameters for the temperature dependence of the NMR shift for $\mathrm{x}=0.13$. . . . . . . . . . . . . . . . . 120

\begin{tabular}{|ll|l|l|l|}
\hline 5.9 & Parameters from fitting the data of figure & $5.25 \mathrm{~b}$ & to $2 \mathrm{D}$ spin \\
\hline
\end{tabular} fluctuations according to equation $[5.1]$. . . . . . . . . . . . 121

5.10 Temperature dependence of $T_{2}$ for both orientations and both components. . . . . . . . . . . . . . . . 123

5.11 The obtained fitting parameters from CW fit of Knight shift. . 126 
5.12 Obtained fitting parameters from a CW fit of the linewidth. . 128

$5.13 \mathrm{~T}_{1}$ for $\mathrm{CeFeAsO}_{0.80} \mathrm{~F}_{0.20}$ at different temperatures and both field orientations with their uncertainties (in brackets). . . . . 129

\begin{tabular}{|ll|l|l|l|}
\hline 5.14 & Fit parameters according to equation & 5.4 & from $1 / T_{1}$ data in \\
\hline
\end{tabular} figure $5.33 \mathrm{~b}$. . . . . . . . . . . . . . . 130

5.15 Temperature dependence of the spin-spin relaxation time, $T_{2} .131$

5.16 Change in quadrupole frequency and lattice spacing in $c \| B_{0} .135$

$5.17 \mathrm{CW}$ fit parameters for the temperature dependent shift of $\mathrm{CeFeAsO}_{1-\mathrm{x}} \mathrm{F}_{\mathrm{x}} \ldots \ldots \ldots \ldots \ldots \ldots . \ldots \ldots 137$

5.18 CW fit parameters for the linewidth. . . . . . . . . . . 138

5.19 Fitting parameters for 2D spin fluctuations (section 5.3 .3 ) to figure 5.40 . . . . . . . . . . . . . . . . . . . 139

6.1 Temperature dependence of the exponent $m$ from a power-law fit (equation $\mid 6.2$ ) to the high-field region of the MR. . . . . . . 151

6.2 Temperature dependence of the exponent $m$ from a power-law fit (equation 6.2) to the high-field region of the magnetoresistance. . . . . . . . . . . . . . . . . . . 154

6.3 Temperature dependence of the exponent $m$ from a power-law fit (equation 6.2 ) to the high-field region of the MR. . . . . . . 156

7.1 The effective moment of different spin configurations of Fe. . . 176 $7.2 \quad T_{C}, \Theta, P_{\text {eff }}^{\text {f.u. }}, \chi_{0}$ and $S(295 \mathrm{~K})$ of $\mathrm{Zn}$ doped Fe1212. . . . . . . 184

8.1 The thermopower for Fe1222 samples at different processing steps. . . . . . . . . . . . . . . . 190 


\section{Chapter 1}

\section{Introduction}

Superconductivity is a very interesting physical phenomenon, where the electrical resistivity goes to zero and the applied magnetic fields are expelled from the sample's core at a finite temperature. Superconductivity, in the low-temperature superconductors, is understood in terms of the (Bardeen, Cooper and Schrieffer) BCS theory [1. However, there remain many unanswered questions in the field of high temperature superconductivity. The research presented here is focused on the investigation of the magnetic and electronic properties of iron-based (iron-pnictides) and iron-cuprate $\oint^{1}$ hybrid superconductors. These compounds cover both groups of high-temperature superconductors while containing a large amount of iron. They also show antiferromagnetic order in the parent compound and interesting multiferroic properties.

The iron-pnictide are second only to the well-studied cuprates in terms of the superconducting transition temperature, $T_{C}$. The superconductivity is based in conducting layers of FeAs, where the iron and arsenic atoms are in tetrahedral bond configuration, and with the arsenic alternating above and below the iron atoms. There is also the intriguing co-existence of superconductivity, spin fluctuations and magnetism spanning almost the entire temperature range in the underdoped region [2 12].

The other group of interesting compounds are the two-dimensional (2D)

\footnotetext{
${ }^{1}$ these are cuprates where every third $\mathrm{Cu}$ is replaced by $\mathrm{Fe}$
} 
iron-cuprate hybrids. They contain a $\mathrm{FeO}_{2-\mathrm{y}}$ deficit layer as well as a $2 \mathrm{D}$ $\mathrm{CuO}_{2}$ layer, which is the superconducting layer. Excess iron is known to be present in the $\mathrm{CuO}_{2}$ layer and the amount can be reduced or increased through different processing steps. The presence of both magnetic as well as superconducting ordering makes these compounds the ideal candidates to study the competing electronic and magnetic interactions. Furthermore, they display a wide variety of interesting physical properties, such as a low temperature spin glass transition and a high negative magnetoresistance in $\mathrm{FeSr}_{2} \mathrm{Y}_{2-\mathrm{x}} \mathrm{Ce}_{\mathrm{x}} \mathrm{Cu}_{2} \mathrm{O}_{10-\mathrm{y}}$ (Fe1222) [13] and a superconducting transition temperature depending on the Fe distribution in $\mathrm{FeSr}_{2} \mathrm{YCu}_{2} \mathrm{O}_{6+\mathrm{y}}(\mathrm{Fe} 1212)$ [14]. There are also no studies covering the electron and hole doping range of Fe1212 and the whole Ce doping range for Fe1222.

The layout of this thesis is as follows. Beginning with chapter 2 the theoretical framework to the electronic and magnetic phenomena encountered in this thesis is described. A short introduction into superconductivity is presented first. The relevant similarities and differences between cuprates and the iron-based superconductors is discussed next, followed by an explanation of the different types of magnetic order observed. A short introduction to spin-glasses is then given before continuing on the variable-rangehopping electronic transport. This is then followed by an introduction to magnetoresistance and the quantum interference magnetoresistance which is applicable to the variable-range-hopping magnetotransport found in the $\mathrm{FeSr}_{2} \mathrm{Y}_{2-\mathrm{x}} \mathrm{Ce}_{\mathrm{x}} \mathrm{Cu}_{2} \mathrm{O}_{10-\mathrm{y}}$. A three-carrier model is also discussed and this is used in chapter 6 to account for the magnetoresistance in the iron-pnictides. Finally a short introduction to nuclear magnetic resonance (NMR) is presented at the end of this chapter.

The experimental details are described in chapter 3. This includes the general sample preparation and the characterization tools used. Particular emphasis is given to describing the electronic transport, magnetotransport and magnetization measurements carried out on the two Quantum Design systems: the Physical Property Measurement System (PPMS) and the Magnetic Property Measurement System (MPMS). The last part of chapter 3 is used to explain the experimental details for the NMR setup. 
Chapter 4 investigates the electronic and magnetic properties of $\mathrm{CeFeAsO}_{1-\mathrm{x}} \mathrm{F}_{\mathrm{x}}$. A detailed description of the synthesis procedure is given and the atomic structure is explained. Results from the temperature dependent resistivity measurements on the non-superconducting $\mathrm{CeFeAsO}_{0.93} \mathrm{~F}_{0.07}$ is presented, followed by the superconducting samples $(x=0.13-0.25)$. The fluctuation conductivity is extracted from the superconducting samples when approaching the superconducting transition temperature. The field dependence of the resistivity is analyzed by means of the thermally activated flux flow (TAFF) model. The doping dependence of the flux flow activation energy, $U_{0}$, and the critical current density, $J_{\mathrm{C}}$, are compared. The temperature and doping dependence of the upper critical field and the irreversible field of the superconducting samples are then presented and analyzed. The last part of this chapter describes the susceptibility measurements and the determination of the effective magnetic moment of the non-superconducting sample; the results of which are used to aid the understanding of the NMR results presented in the next chapter.

Chapter 5 focuses on the NMR measurements on the same materials as in chapter 4 . These measurements were done under a magnetic field of $11.74 \mathrm{~T}$ both parallel and perpendicular to the c-axis of the aligned sample. The investigation begins with the parent compound $\mathrm{CeFeAsO}$ without fluoride doping. The temperature dependence of the NMR spectrum shows the antiferromagnetic ordering due to the spin-density-wave (SDW) transition and it allows an estimation of the internal field strength of this ordering. Spinlattice relaxation and spin-spin relaxation are studied next. The $\mathrm{x}=0.07$ nominal fluoride doped sample is studied first, where the spin-density-wave is vanishing and on the verge of becoming superconducting. The temperature dependence of the NMR spectra, linewidth, shift, spin-lattice relaxation and spin-spin relaxation were investigated in both field directions. Similar measurements were done on the superconducting compounds $\mathrm{CeFeAsO}_{0.87} \mathrm{~F}_{0.13}$ and $\mathrm{CeFeAsO}_{0.80} \mathrm{~F}_{0.20}$. By comparing all these measurements the doping dependence of the NMR properties of $\mathrm{CeFeAsO}_{1-\mathrm{x}} \mathrm{F}_{\mathrm{x}}$ is extracted.

Chapter 6 is dedicated to a different type of iron-based superconductor. While chapter 4 and 5 are focused on one member of the 1111-family, 
in this chapter two members of the 122-family, namely $\mathrm{AFe}_{2} \mathrm{As}_{2}(\mathrm{~A}=\mathrm{Ba}$ or $\mathrm{Sr})$ were investigated. This is motivated by the appearance of a resistivity downturn in these samples at low temperatures, which indicates a developing superconducting state [15, 16]. The temperature dependent resistivity and magnetoresistance are presented for $\mathrm{BaFe}_{2} \mathrm{As}_{2}, \mathrm{SrFe}_{2} \mathrm{As}_{2}$ and $0.8 \mathrm{at} \% \mathrm{Ca}$ implanted $\mathrm{SrFe}_{2} \mathrm{As}_{2}$. The magnetoresistance is analyzed with both linear quantum magnetoresistance and a three-carrier model. This is followed by a description of an aging effect in $\mathrm{SrFe}_{2} \mathrm{As}_{2}$, in which it becomes superconducting after being held in a vacuum desiccator for seven months, and displays significant changes to the resistivity and magnetoresistance. This process is reversible by annealing in an Argon atmosphere.

Chapter 7 presents the study of the effect of electronic doping on the magnetic, electronic and vibrational properties of $\mathrm{FeSr}_{2} \mathrm{YCu}_{2} \mathrm{O}_{6+\mathrm{y}}$ known as Fe1212. A detailed synthesis is described and the structure is compared to the well-known structure of $\mathrm{RuSr}_{2} \mathrm{RCu}_{2} \mathrm{O}_{6+\mathrm{y}}$ ( $\mathrm{R}$ is a rare earth or $\mathrm{Y}$ ). Results from magnetization measurements are shown including the changes in the superconducting transition temperature, the room temperature thermopower and the temperature independent part of the susceptibility. This is followed by the Curie-Weiss (CW) temperature dependence with electron (La) and hole ( $\mathrm{Ca}$ ) doping. In addition to these measurements the effect of $\mathrm{Zn}$ substitution for $\mathrm{Cu}$ on the superconducting properties were also studied.

The magnetic and magnetotransport properties of $\mathrm{FeSr}_{2} \mathrm{Y}_{2-\mathrm{x}} \mathrm{Ce}_{\mathrm{x}} \mathrm{Cu}_{2} \mathrm{O}_{10-\mathrm{y}}$ are investigated in chapter 8 , in regard to the spin and electronic disorder. The synthesis and the structure is described, before presenting the magnetization where an interesting spin glass behavior was observed. This is followed by a study of the doping dependence of the susceptibility, where the effective moment is calculated from a Curie-Weiss temperature dependent fit. The second part of this chapter covers the temperature dependence of the resistivity and the magnetoresistance. The last part of the chapter presents the changes that occur in effective moment, magnetic ordering and electronic transport when the sample is in the oxygen reduced and the oxygen saturated state.

The last chapter summarizes the presented work on the iron-cuprate hy- 
brid and the iron-based superconductors. Then an overall conclusion is given and a short outlook of possible further work. 


\section{Chapter 2}

\section{Theory}

Chapter 2 provides the theoretical framework as well as an introduction into superconductivity. This includes a historical overview of superconductivity and an explanation of a few important concepts. The relevant similarities and differences between cuprates and the iron-based superconductors will be discussed next, followed by an explanation of the antiferromagnetic Mott insulating state observed in the cuprates and the antiferromagnetic spin-densitywave (SDW) ordered state observed in the iron-based superconductors. A short introduction to spin-glasses is given before continuing to the electronic transport where variable-range-hopping (VRH) will be presented. This is followed by an introduction to magnetoresistance (MR) and the applicable models of quantum interference (QI) magnetoresistance and the three-carrier model. Finally, there is a short introduction to nuclear magnetic resonance (NMR). 


\subsection{Superconductivity}

The materials under investigation are all either superconducting or have superconductivity suppressed by iron-copper anti-site disorder. Hence, it seems appropriate to begin with a short overview of superconductivity starting with the history of superconductivity. This is followed by a discussion of the concept of the superconducting transition temperature, $T_{\mathrm{C}}$, the critical magnetic field, $B_{\mathrm{C}}$, and the critical current density, $J_{\mathrm{C}}$. Further information can be found for example, in "Introduction to Superconductivity" by Michael Tinkham [17], and Ashcroft and Mermin's "Solid State Physics" [18].

\subsubsection{Historical overview}

The history of superconductivity starts with Heike Kamerlingh Omnes, who discovered superconductivity in 1911 in his laboratory in Leiden [19 21]. This discovery was made possible by his earlier achievement in 1908 where he liquefied Helium for the first time. He used this to study the low temperature resistivity of mercury, where he observed a sudden drop in resistivity at $4.2 \mathrm{~K}$. One of his later experiments used a superconducting ring of mercury wire, in which a persistent current flowed for over a year. He was awarded the Nobel Prize in 1913 for his development of the liquefaction of helium rather than his discovery of superconductivity.

The next big step was made by W. Meissner and R. Ochsenfeld with their discovery of the exclusion of the magnetic field later called the Meissner effect [22]. A superconductor does not allow the magnetic field to penetrate into it through induced screening currents which create a magnetic field opposite of the external field. This leads to the most popular demonstration of superconductivity by levitating a magnet above a superconductor or vice versa. However, this stays true until the critical magnetic field, $B_{\mathrm{C}}$ for a type I superconductor is reached and the magnetic field starts to penetrate into the superconductor (see figure 2.2 for more details), which destroys superconductivity.

John Bardeen, Lean Cooper and Robert Schrieffer developed in 1957, at the University of Illinois, a model for superconductivity [1]. This model states 
that electrons in a certain energy range close to the Fermi level can form Cooper pairs out of 2 electrons with opposite spin under a constant pairing potential. These Cooper pairs have zero spin in the quantum ground state, where the coherence can be as large as $1000 \AA$ in some type I superconductors and as small as a few $\AA$ (smaller than their inter-layer distance) in some type II superconductors. The Nobel Prize in physics was awarded to them in 1972 for the development of this theory of superconductivity called the BCS theory.

A rather unexpected discovery was made 1986 by Alex Müller and Georg Bednorz while working on metal oxide ceramics (perovskite). They found that the resistivity of $\mathrm{La}_{2-\mathrm{x}} \mathrm{Ba}_{\mathrm{x}} \mathrm{CuO}_{4}$ drops to zero around $35 \mathrm{~K}$ indicating superconductivity [23]. This was quite astonishing, because up to that time the highest known superconducting transition temperature was $22.3 \mathrm{~K}$ in $\mathrm{Nb}_{3} \mathrm{Ge}$ [24]. This triggered a race around the world to look for even higher $T_{\mathrm{C}}$ in similar kinds of materials, which were found over the following years with $T_{\mathrm{C}}$ higher than liquid nitrogen $(77 \mathrm{~K})$. Examples of these copper-based oxides include $\mathrm{YBa}_{2} \mathrm{Cu}_{3} \mathrm{O}_{6+\mathrm{y}}$ (YBCO is the parent compound of the iron cuprate compound studied in chapter 7 and 8 with a $T_{\mathrm{C}}$ of $92 \mathrm{~K}$ [25], $\mathrm{Bi}_{2} \mathrm{Sr}_{2} \mathrm{Ca}_{2} \mathrm{Cu}_{3} \mathrm{O}_{10+\mathrm{y}}$ [26] (BSCCO) with a $T_{\mathrm{C}}$ of $108 \mathrm{~K}$ and $\mathrm{HgBa}_{2} \mathrm{Ca}_{2} \mathrm{Cu}_{3} \mathrm{O}_{8+\mathrm{y}}$ [27] with the highest observed $T_{\mathrm{C}}$ of $134 \mathrm{~K}$ so far at ambient pressure ${ }^{1}$.

In February 2008 Hideo Hosono and colleagues at Tokyo Institute of Technology discovered that $\mathrm{LaFeAsO}_{1-\mathrm{x}} \mathrm{F}_{\mathrm{x}}$ was superconducting at $26 \mathrm{~K}$ [29]. The new iron-based family of high temperature superconductors vastly expanded over the next few months with the highest $T_{\mathrm{C}}$ observed in the oxypnictides (1111-group) with $\mathrm{SmFeAsO}_{1-\mathrm{x}}$ at $55 \mathrm{~K}$ [30] and the oxygen free variant of $\mathrm{Sr}_{0.5} \mathrm{Sm}_{0.5} \mathrm{FeAsF}$ at $56 \mathrm{~K}$ [31]. Other related groups are the 122-group with a maximum $T_{\mathrm{C}}$ of $38 \mathrm{~K}$ in $\mathrm{Ba}_{1-\mathrm{x}} \mathrm{K}_{\mathrm{x}} \mathrm{Fe}_{2} \mathrm{As}_{2}$ [32], 111-group like NaFeAs [33, 34] reaching $18 \mathrm{~K}$ and 11 -family with FeSe having a $T_{\mathrm{C}}$ of $14 \mathrm{~K}$ [35, 36.

\footnotetext{
${ }^{1}$ the higher $T_{\mathrm{C}}$ is achieved for $\mathrm{HBCOO}$ with $164 \mathrm{~K}$ under high pressure. Just recently $\mathrm{H}_{2} \mathrm{~S}$ was found to be superconducting under high pressure (around $200 \mathrm{GPa}$ up to a $T_{\mathrm{C}}$ of $190 \mathrm{~K} 28$.

${ }^{2}$ much higher $T_{\mathrm{C}}$ have been reported for single layer FeSe 37 .
} 


\subsubsection{The critical temperature}

The critical superconducting transition temperature, $T_{\mathrm{C}}$, is the temperature at which a material becomes superconducting. However, in practice there exist many ways to define $T_{\mathrm{C}}$ experimentally. The four more common methods are shown in figure 2.1 with $T_{\mathrm{C}}^{\mathrm{I}}$ as the beginning of a downturn in resistivity, $T_{\mathrm{C}}^{\mathrm{II}}$ at $90 \%$ of the normal state resistivity, $T_{\mathrm{C}}^{\mathrm{III}}$ the intersection between the slope of the resistivity from above (red line) and during (orange line) the transition and $T_{\mathrm{C}}^{\mathrm{IV}}$ when zero resistivity is reached. This complexity comes from the fact that a superconducting transition is an effect, which often stretches over a long temperature range despite being well defined theoretically. The width of such a transition (for example, from $T_{\mathrm{C}}^{\mathrm{I}}$ to $T_{\mathrm{C}}^{\mathrm{IV}}$ ) is often broadened by vortex pinning and fluctuation conductivity, which makes it difficult to determine an exact $T_{\mathrm{C}}$.

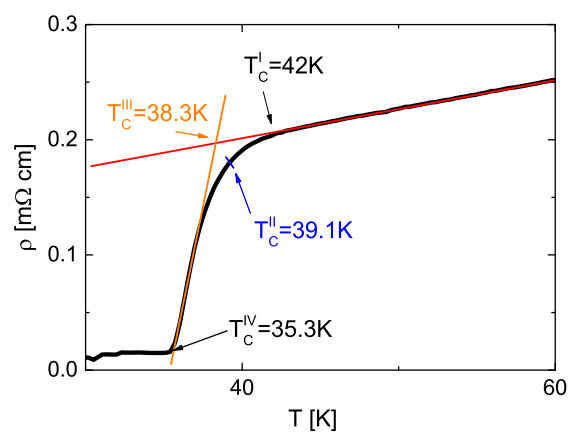

Figure 2.1: Four different ways to determine $T_{\mathrm{C}}$.

\subsubsection{The critical magnetic field}

The critical magnetic field, $B_{\mathrm{C}}$, is the magnetic field at a given temperature, where superconductivity is suppressed. This is only the case for conventional superconductivity found in the type I superconductors (for example, $\mathrm{Hg}$ and $\mathrm{Al}$ ). The high temperature superconductors (for example, cuprates and ironbased) are all type II where two different critical magnetic fields exist. The lower critical field, $B_{\mathrm{c} 1}$, is the field at which at a given temperature the magnetic field starts to penetrate the superconductor in the form of vortices, 
which all contain exactly one magnetic flux quantum, $\Phi_{0}=h / 2 e$ [38]. The number density of vortices increases with increasing applied field until a certain upper critical field, $B_{\mathrm{c} 2}$, at which superconductivity is completely destroyed just as in the type I superconductors. The temperature dependence of these critical fields can be seen in figure 2.2 .

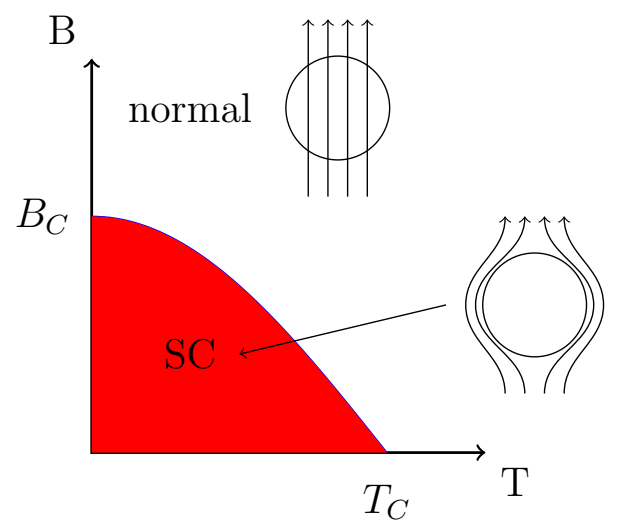

(a) Type I superconductor

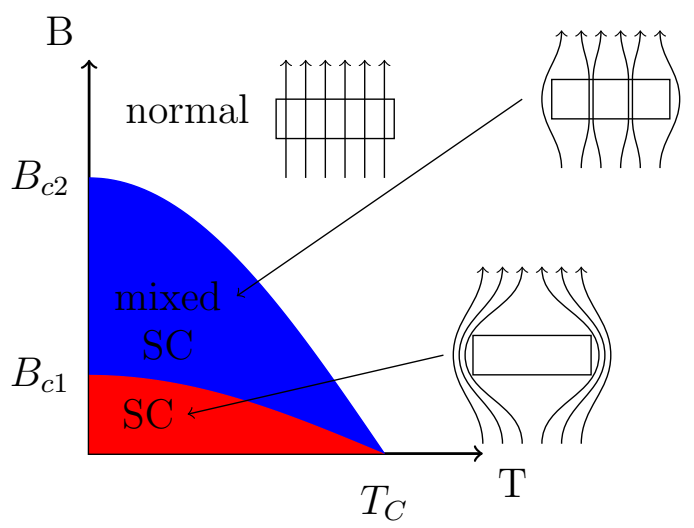

(b) Type II superconductor

Figure 2.2: The temperature dependence of the critical magnetic fields of type I and type II superconductors.

The vortices penetrating the superconductors are free to move in the direction of the magnetic field. This leads to dissipation of energy and heating, both unwanted effects in a superconductor. This can be prevented if the vortices are pinned, which happens naturally on crystal defects or can be achieved artificially with the introduction of dedicated impurities and defects. An easy way to distinguish between these two types of superconductors is made possible within the Ginzburg-Landau (GL) theory of superconductors [39]. The GL theory introduces the GL coherence length [17, 39],

$$
\xi(T)=\frac{\hbar}{\left|2 m^{*} \alpha(T)\right|^{1 / 2}}
$$

where $\hbar$ is the reduced Planck constant, $m^{*}$ is the mass and $\alpha(T)$ is an expansion coefficient of $\nabla \psi$, where $\psi$ is a pseudo wave function within the GL theory. The London brothers developed the London equations [40] in 1935 from which the London penetration depth can be derived [17, 


$$
\lambda_{\mathrm{L}}(0)=\sqrt{\frac{m^{*}}{\mu_{0} n e^{2}}}
$$

where $\mu_{0}$ is the vacuum permeability, $n$ is the number density of superconducting electrons and $e$ the elemental charge. The GL parameter is defined as the ratio of those two characteristic lengths,

$$
\kappa=\frac{\lambda}{\xi}
$$

Both lengths diverge as $1 / \sqrt{\left(T-T_{\mathrm{C}}\right)}$ near $T_{\mathrm{C}}$, which makes $\kappa$ approximately temperature independent [17]. Abrikosov [38] showed in 1957 that the crossover between type I and type II occurs at $\kappa=1 / \sqrt{2}$ with typical type I superconductors having $\kappa \ll 1$.

\subsubsection{The critical current density}

Another important property for practical applications is the critical current density, $J_{\mathrm{C}}$. For a type I superconductor it is possible to derive a superconducting critical current based on the assumption that when the field created by the superconducting critical current is greater than $B_{\mathrm{C}}$, then superconductivity is completely suppressed. This is called the Silsbee criterion for type I superconductors and for a long superconducting wire with a radius, $r \gg \lambda,[17$ :

$$
I_{\mathrm{C}}=\frac{2 \cdot \pi \cdot r \cdot B_{\mathrm{C}}}{\mu_{0}}
$$

The current in a superconducting wire only flows in a surface layer of thickness equal to the London penetration depth $\lambda_{\mathrm{L}}$. This leads to a critical current density [17],

$$
J_{\mathrm{C}}=\frac{2 \cdot B_{\mathrm{C}}}{\mu_{0} \cdot r \cdot \lambda_{\mathrm{L}}}
$$




\subsection{Comparison between cuprates and iron- based superconductors}

Both the cuprates and iron-based superconductors have obvious similarities, but also significant differences. To highlight just a few, which are relevant to this thesis, one could begin by examining the phase diagram from each group of superconductors. Figure 2.3a shows the generic phase diagram of a cuprate from the 214-type, figure 2.3b shows the phase diagram of iron-based $\mathrm{CeFeAsO}_{1-\mathrm{x}} \mathrm{F}_{\mathrm{x}}(\mathrm{Ce} 1111)$ and $2.3 \mathrm{c}$ the phase diagram of 122-type pnictide and chalcogenide. Both groups develop superconductivity by adding additional charge carriers to an antiferromagnetically ordered parent compound. However, the detailed antiferromagnetic ordering in both parent compounds is different. The parent compound of the cuprates is an antiferromagnetic (AFM) Mott insulator, while the parent compound of the iron-based superconductors is a metal with an AFM spin-density-wave phase. Such differences continue for the doped materials, despite both containing $3 d$ transition metals and a basic structure is built out of 2-dimensional (2D) planes. The superconducting planes of the cuprates are the $\mathrm{CuO}_{2}$ planes, which can be described by a single band model involving the $\mathrm{Cu} 3 \mathrm{~d}_{x^{2}-y^{2}}$ orbital and the oxygen $2 p$ orbital [41]. For the iron-based superconductors however, all five $3 d$ orbitals of Fe contribute to the states around the Fermi level and hence a multi-band model is necessary [42 45].

The transport properties of both materials show similarities in the normal state. For example, the resistivity shows a Fermi-liquid like $T^{2}$ behavior in the overdoped region [46, 47]. This temperature dependence changes to a linear dependency close to optimal doping suggesting a non-Fermi-liquid state sometimes referred to as "strange metal" [48 52]. 


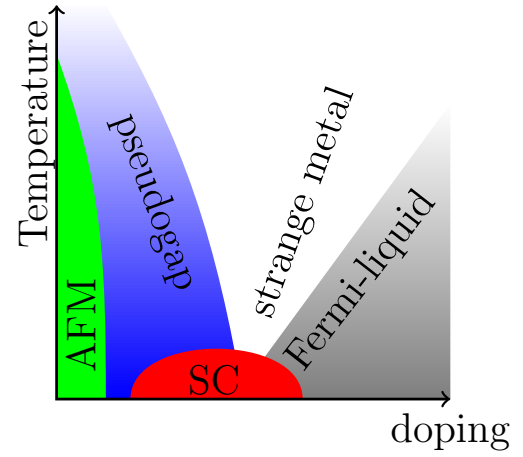

(a) Typical cuprate

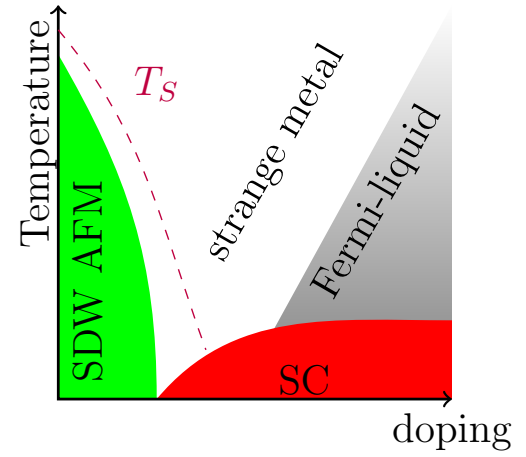

(b) Ce1111

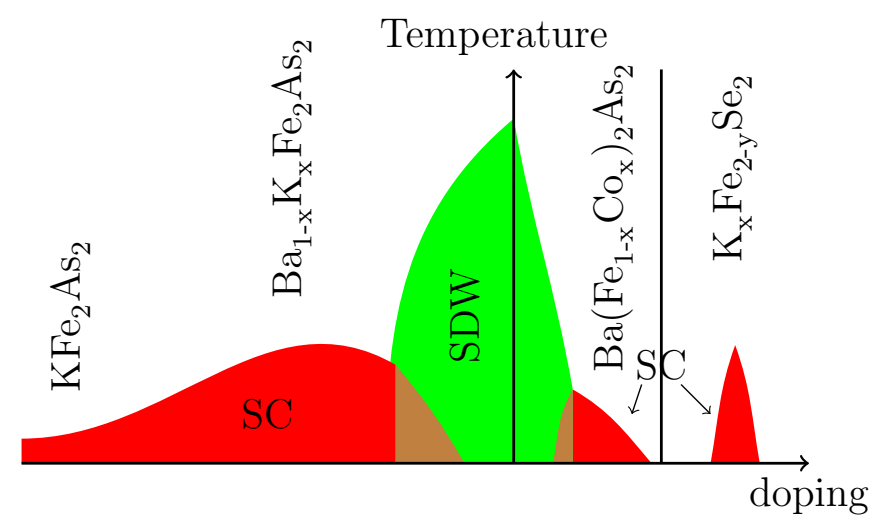

(c) Ba122

Figure 2.3: The phase diagram of cuprates and iron-based superconductors. 


\subsection{Magnetic order}

This section discusses magnetic order relevant to this thesis. Further details and other types of magnetic order can be found in standard textbooks of condensed matter physics [18, 53]. Hence, only a few special forms of magnetic order encountered in high temperature superconductors will be mentioned in this section.

\subsubsection{Antiferromagnetic spin-density-wave order}

Most iron-based superconductors have a low temperature spin-density-wave (SDW) ordering in the parent compound. For example, the spins in the SDW ordered state can have an antiferromagnetic spin alignment along one crystal axis and a ferromagnetic alignment along the other which is called co-linear antiferromagnetic order, as shown in figure 2.4 and are observed in La1111 [54, 55], Ce1111 [2, 56], Pr1111 [3] and Nd1111 [57].

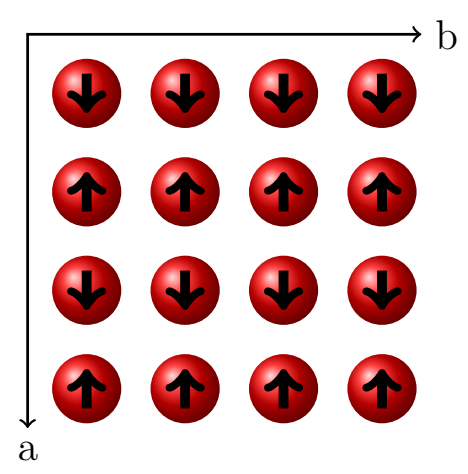

Figure 2.4: Depiction of the co-linear antiferromagnetic order if the Fe atoms in the spin-density-wave state commonly observed in the 1111- and 122-family of the iron-based superconductors.

The SDW transition is always observed at a temperature that is slightly lower than the structural transition. When these materials are cooled down from room temperature they reach the structural transition first, which changes the structure from the tetragonal space group $P 4 / \mathrm{nmm}$ to a monoclinic $P 112 / n$ or orthorhombic $C m m a$ in the temperature range of $160 \mathrm{~K}$ 
to $150 \mathrm{~K}$ [2, 3, 54, 55, 57]. The structural transition is followed by a longrange SDW ordering with a co-linear spin structure (figure 2.4 below $145 \mathrm{~K}$ [2, 3, 55, 58]. Similar behavior, a structural transition followed by an AFM order, also occurs in the 214-type cuprates [59]. The observed AFM order in different rare-earth oxypnictides is suggested to arise from a spin-densitywave (SDW) instability from a nested Fermi surface [5, 42, 54, 60]. However, it remains unclear how the difference in the band structure can explain the different observed AFM magnetic structures and moments of Fe in the oxypnictides [2]. 


\subsubsection{Antiferromagnetic Mott insulator}

The parent compounds of many cuprates are antiferromagnetic Mott insulators [61. They are similar too a number of metal oxides, which should be metallic under conventional band theory, but they are insulators when they are measured. Nevill F. Mott 62] proposed that this discrepancy can be explained by including interactions between electrons and in particular via the Coulomb interaction. This results in the opening of a gap in the density of states and leads to insulating behavior.

The simplest theoretical model explaining the metal-to-insulator transition (MIT) was developed by Hubbard [63 66] in 1964 and is now called the Hubbard model. This model is based on the tight-binding approximation, which approximates electrons as occupying the standard orbitals of their constituent atoms. The electrons then hop between atoms during conduction described by a hopping matrix term, $t$. The Hubbard model considers only electrons in a single band and includes the on-site Coulomb repulsion as an additional term, $U$. The Hamiltonian ${ }^{3}$ is given by

$$
\begin{gathered}
H_{\mathrm{H}}=H_{\mathrm{t}}+H_{\mathrm{U}}-\mu N \\
H_{t}=-t \sum_{\langle i j\rangle}\left(c_{i \sigma}^{\dagger} c_{j \sigma}+c_{j \sigma}^{\dagger} c_{i \sigma}\right) \\
H_{U}=U \sum_{i}\left(n_{i \uparrow}-\frac{1}{2}\right)\left(n_{i \downarrow}-\frac{1}{2}\right) \\
N=\sum_{i \sigma} n_{i \sigma}
\end{gathered}
$$

where $\mu$ is the chemical potential, $c_{i \sigma}^{\dagger}\left(c_{i \sigma}\right)$ is the creation (annihilation) operator of the electron at site $i$ and with spin $\sigma$, while $n_{i \sigma}=c_{i \sigma}^{\dagger} c_{i \sigma}$ is the number operator. This requires that the relevant low energy excitations be described by a single band near the Fermi surface. Such a description is

\footnotetext{
${ }^{3}$ in second quantized form
} 
possible with an additional assumption that the orbital degeneracy is lifted through the strong anisotropic crystal field and that the ligand $p$-band is either far enough away or strongly hybridized. Furthermore, the potential is the on-site Coulomb repulsion and the inter-site effects are neglected. Despite the many simplifications used in creating this model is it successful in recreating the transition between a Mott insulator to a metal.

Further, models [67] were subsequently developed based on the Hubbard model through the inclusion of additional terms for the nearest-neighbor interaction (which can explain the charge ordering effect). Cases where more than one $d$-orbital have to be considered lead to the degenerate Hubbard model. But in the case of transition metals such as $\mathrm{Cu}$ where all $d$-orbitals are filled, except the $d_{x^{2}-y^{2}}$ band that is half-filled, making the assumption of a single band still valid. The later case was first described by K.A. Chao, J. Spalek and A.M. Oles [68] in 1977 by a second order perturbation in $t / U$ to the Hubbard model, $J$, leading to the $t-J$ model [68 70] with the following Hamiltonian,

$$
H_{t-J}=-t \sum_{\langle i j\rangle, \sigma} P_{d}\left(c_{i \sigma}^{\dagger} c_{j \sigma}+c_{j \sigma}^{\dagger} c_{i \sigma}\right) P_{d}+J \sum_{\langle i j\rangle}\left(S_{i} S_{j}\right)
$$

where the double occupied sites are excluded through the projection operator $P_{d}$ and $S_{i}, S_{j}$ are the spins at sites $i$ and $j$.

\subsubsection{Spin Glasses}

The special form of magnetic order observed here is a spin glass state for the doped Fe1222 compound. A spin glass [71 75] does not show long range magnetic order and it can occur when there is either a randomness in the position of the spins or a randomness in the sign of neighboring interactions. This means there needs to be either site or bond disorder to create a spin glass. For such a system there exists a characteristic temperature, $T_{f}$, called the freezing temperature. Below this temperature there develops a frozen irreversible metastable state. The dynamics of such a system is commonly described by a frequency dependence of the spin glass state with changing 
temperature through the Vogel-Fulcher equation [76 80]:

$$
\tau\left(T_{\mathrm{f}}\right)=\tau_{0} \exp \left[\frac{E_{\mathrm{a}}}{k_{\mathrm{B}}\left(T_{\mathrm{f}}-T_{1}\right)}\right]
$$

where $E_{\mathrm{a}}$ is the activation energy, $k_{\mathrm{B}}$ is Boltzmann's constant and $T_{1}$ is a phenomenological parameter of the Vogel-Fulcher law for spin-glasses [80]. The phenomenological parameter is introduced as a measure of the interaction strength within spin clusters to fit the data. The frequency dependence of $\tau$ can be obtained from AC magnetization measurements. 


\subsection{Electronic Transport}

In this section several models used to explain the electronic transport properties of the investigated materials in this thesis are described. Most high temperature superconductors have a Fermi-liquid phase in the overdoped region of their doping phase diagram, where the resistivity follows a $T^{2}$-behavior. Detailed descriptions of the Fermi-liquid state can be found in Ashcroft and Mermin [18] and references therein, as well as in these references [81,83].

The more interesting transport behavior is at the lower temperature region for highly disordered systems. Under such circumstances variable-rangehopping (VRH) can occur, which was first described by Mott [84, 85]. When $\mathrm{VRH}$ is the dominant conduction mechanism, the percolation pathway is determined by two competing types of conduction, which are tunneling, defined by $\exp (-2 R / \xi)$, and thermal activated hopping, defined by $\exp \left(W / k_{\mathrm{B}} T\right)$. The resultant hopping rate between the two states can be written as:

$$
\frac{1}{\tau_{\mathrm{h}}}=\frac{1}{\tau_{\mathrm{h}, 0}} \exp \left(-\frac{2 D}{\xi}-\frac{W}{k_{\mathrm{B}} T}\right)
$$

where $D$ is the distance between the two localized states, $\xi$ is the localization radius, $W$ is the energy difference between the two localized states, and $1 / \tau_{\mathrm{h}, 0}$ is a constant that includes the attempt frequency. Mott then derived the conduction of the transport process through a single critical hop leading to the Mott conductivity:

$$
\sigma=\sigma_{0} \exp \left(-\left(\frac{T_{0}}{T}\right)^{p}\right)
$$

where $T_{0}$ is a characteristic temperature of the system and $p$ a characteristic exponent which differs for different conduction mechanisms. Mott assumed a constant density of states (DOS) near the Fermi energy, $N\left(E_{\mathrm{F}}\right)$, which leads to $p=1 / 4$ and $1 / 3$ for $3 \mathrm{D}$ and $2 \mathrm{D} \mathrm{VRH}$ hopping, respectively. The characteristic temperature in d dimensions is then given by

$$
T_{0}=\frac{1}{k_{\mathrm{B}} N\left(E_{\mathrm{F}}\right) \xi^{d}}
$$


Efros and Shklovskii [86] showed later that the DOS is not constant when Coulomb interactions are taken into consideration. However, equation 2.8 is still valid in this case with $p=1 / 2$ for all dimensions. The characteristic temperature in $2 \mathrm{D}$ and $3 \mathrm{D}$ is given by

$$
T_{0}=\frac{2.8 e^{2}}{4 \pi \epsilon \epsilon_{0} k_{\mathrm{B}} \xi}
$$

where $\epsilon_{0}$ is the permittivity of vacuum, $\epsilon$ is the dielectric constant of the material and $e$ the elementary charge. It is possible depending on the disorder of the system and the temperature to only see a $2 \mathrm{D}$ or $3 \mathrm{D}$ dependence, or a crossover from $2 \mathrm{D}$ to $3 \mathrm{D}$ or vice versa. 


\subsection{Magnetoresistance}

Magnetoresistance is an effect where the resistivity of the material changes depending on the applied magnetic field. The magnetoresistance, $M R(B)$, can be defined as,

$$
M R(B)=\frac{\rho(B)-\rho(0)}{\rho(0)}
$$

where $\rho(B)$ is the resistivity at an applied magnetic field, $B$, and $\rho(0)$ is the resistivity for $B=0$. There are several different magnetoresistance effects including the original ordinary MR discovered by Lord Kelvin 1856 as well as the later discoveries of giant and colossal MR, tunnel MR and extraordinary MR. This section will mention the basic mechanism of several types of commonly encountered magnetoresistance and then continue with a detailed description of the variable-range-hopping quantum interference (VRH QI) model and the three-carrier formalism used in this work.

Ordinary MR [87] is the simplest form of magnetoresistance, which is due to the Lorentz force acting on the applied current. This usually means that a magnetic field perpendicular to the current increases the resistance of the material and results in a positive MR. This positive MR usually follows a quadratic field dependent behavior. Extraordinary MR is a geometrical MR effect associated with an extreme rise in resistance with the application of a large transverse magnetic field in metal-semiconductor hybrid systems. This effect is due to the large magnetic field causing the Hall angle to approach $90^{\circ}$, with the current flow inside the metallic region dramatically reduced. The system's geometry has a strong effect on this type of MR [88, 89].

A number of compounds are known to show an anisotropic magnetoresistance where there is a difference in the resistance depending on whether the magnetization is parallel or perpendicular to the applied magnetic field. Anisotropic MR can occur in some $3 d$ transition metals (e.g. Ni, Fe), where there is s-d scattering and a spin orbit interaction results in scattering in between the up-spin and the down-spin $d$ states [90]. The net results is that the resistance is greatest when the magnetization is parallel to the current 
[87, 90, 91].

Giant MR [92, 93] is observed in thin films of electronically spin polarized materials, where the magnetoresistance depends on the magnetization alignment of the spins in adjacent ferromagnetic layer (for example, Co) separated by a nonmagnetic layer (for example, $\mathrm{Cu}$ ). Such an arrangement leads to low resistance for parallel and large resistance in an antiparallel arrangements and can be controlled by the magnetic field because of the spin scattering rate dependence on the spin orientation. Another form of giant MR occurs in magnetic tunnel junctions through spin tunneling [94] also called spin tunneling MR. These magnetic tunnel junctions consist of two electronically spin polarized ferromagnetic layers with an insulating layer in between. If the insulating layer is thin enough (a few $\mathrm{nm}$ ) then electrons can travel from one side to the other through quantum mechanical tunneling. The MR in such a device occurs because the tunneling favors a parallel orientation of the two ferromagnetic layers. The orientation of the magnetization in these layers can be changed by the magnetic field and hence the MR also. 


\subsubsection{Quantum interference magnetoresistance}

Quantum Interference (QI) MR occurs in weakly localized systems where there is forward and back scattering in a self-intersecting loop that results in a negative MR [95, 96]. It can also occur in strongly localized materials and in the $2 \mathrm{D}$ and $3 \mathrm{D} \mathrm{VRH}$ regimes if there are many elastic scattering centers between the localized hopping sites [97, 98]. This can lead to different tunneling paths in a "cigar-shaped" 4 region that connects two hopping sites where interference of the tunneling amplitudes can result in an orbital MR in the presence of an applied magnetic field [97, 98]. Unlike the weakly localized case, VRH QI occurs only for forward scattering events because back scattering provides a negligible contribution to the orbital MR. The requirement for many scattering centers between the hopping sites means that this type of quantum interference MR is observed at low temperatures and where the average hopping distance, $\widetilde{R}$ [84], is large.

$$
\widetilde{R}=\left[\frac{T_{0}}{T}\right]^{n} \xi
$$

If the scattering amplitudes are positive then it is possible to have quantum interference and a positive MR [97, 99 104]. A negative MR can occur if there is randomness in the scattering amplitude [97, 98, 105-109] with a typical field dependence of $\sim B^{2}$ at low fields and linear at high fields.

\footnotetext{
4 "cigar-shaped" = so called in literature, region which contributes to relevant scattering amplitude
} 


\subsubsection{Three-carrier formalism}

For systems such as the iron-based superconductors, where more than one band crosses the Fermi surface, multiple carriers can be involved in the transport, which can have different behaviors. Hence, attempts to explain such system with just one model often fail and a multi-carrier approach is needed. Such formalisms were developed for semiconductors using a reducedconductivity-tensor (RCT) scheme [110] with different carrier components, $J$.

Kim [111] has developed a three-carrier matrix formalism for the longitudinal MR under which the MR can be written as,

$$
M R=\frac{\left[\left(\alpha+\gamma B^{2}\right) B^{2}\right]}{\left[1+\left(\beta+\delta B^{2}\right) B^{2}\right]}
$$

where

$$
\begin{gathered}
\alpha=f_{1} f_{2}\left(\mu_{1}-\mu_{2}\right)^{2}+f_{1} f_{3}\left(\mu_{1}-\mu_{3}\right)^{2}+f_{2} f_{3}\left(\mu_{2}-\mu_{3}\right)^{2} \\
\gamma=f_{1} f_{2}\left(\mu_{1}-\mu_{2}\right)^{2} \mu_{3}^{2}+f_{1} f_{3}\left(\mu_{1}-\mu_{3}\right)^{2} \mu_{2}^{2}+f_{2} f_{3}\left(\mu_{2}-\mu_{3}\right)^{2} \mu_{1}^{2} \\
\beta=\left(f_{1} \mu_{2}+f_{2} \mu_{1}\right)^{2}+\left(f_{1} \mu_{3}+f_{3} \mu_{1}\right)^{2}+\left(f_{3} \mu_{2}+f_{2} \mu_{3}\right)^{2} \\
\delta=\left(f_{1} \mu_{2} \mu_{3}+f_{2} \mu_{1} \mu_{3}+f_{3} \mu_{1} \mu_{2}\right)^{2} \\
f_{i}=\frac{\left|n_{i} \mu_{i}\right|}{\sum\left|n_{i} \mu_{i}\right|}
\end{gathered}
$$

and $n_{i}$ is the carrier density while $\mu_{i}$ is the carrier mobility. This model is valid for different numbers of carriers $J$, however the MR for $J=1$ would be zero in an ideal case. 


\subsection{Nuclear Magnetic Resonance}

NMR uses the splitting of the energy levels of the nuclear spin, $I$, in an external magnetic field, $B_{0}$, (Zeeman effect) and excites these energy difference with resonant radio frequency pulses. These frequencies are a unique fingerprint of the nucleus and it surroundings and hence offer a non-invasive method to study static and dynamic effects. A good overview about NMR can be found in the book of C.P. Slichter "Principles of Magnetic Resonance" [112].

NMR is a very good tool for studying high temperature superconductors because most nuclei contained in them either have $I \geq 1 / 2$ (for example, ${ }^{63} \mathrm{Cu}$ and ${ }^{75}$ As have $I=3 / 2$ ) or it is possible to exchange them for other isotopes that do (for example, ${ }^{16} \mathrm{O}$ has $I=0$, but ${ }^{17} \mathrm{O}$ has $I=5 / 2$ ). However, NMR in this work is focused on a systematic study of ${ }^{75} \mathrm{As}$ in $\mathrm{CeFeAsO}_{1-\mathrm{x}} \mathrm{F}_{\mathrm{x}}$ (chapter 5). ${ }^{75}$ As has a nuclear spin of $I=3 / 2$ and this means that quadrupole interactions play a major role in the spectra. Figure 2.5 shows the energy level of such a nuclei under an applied magnetic field (Zeeman) and then the further shifts due to quadrupole interactions treated as first and second order perturbations. In $\mathrm{CeFeAsO}_{1-\mathrm{x}} \mathrm{F}_{\mathrm{x}}$ the first order quadrupole perturbation is of the order of $10 \mathrm{MHz}$ to $11 \mathrm{MHz}$. It is possible to directly observe this without an applied magnetic field in nuclear quadrupole resonance (NQR) measurements. 


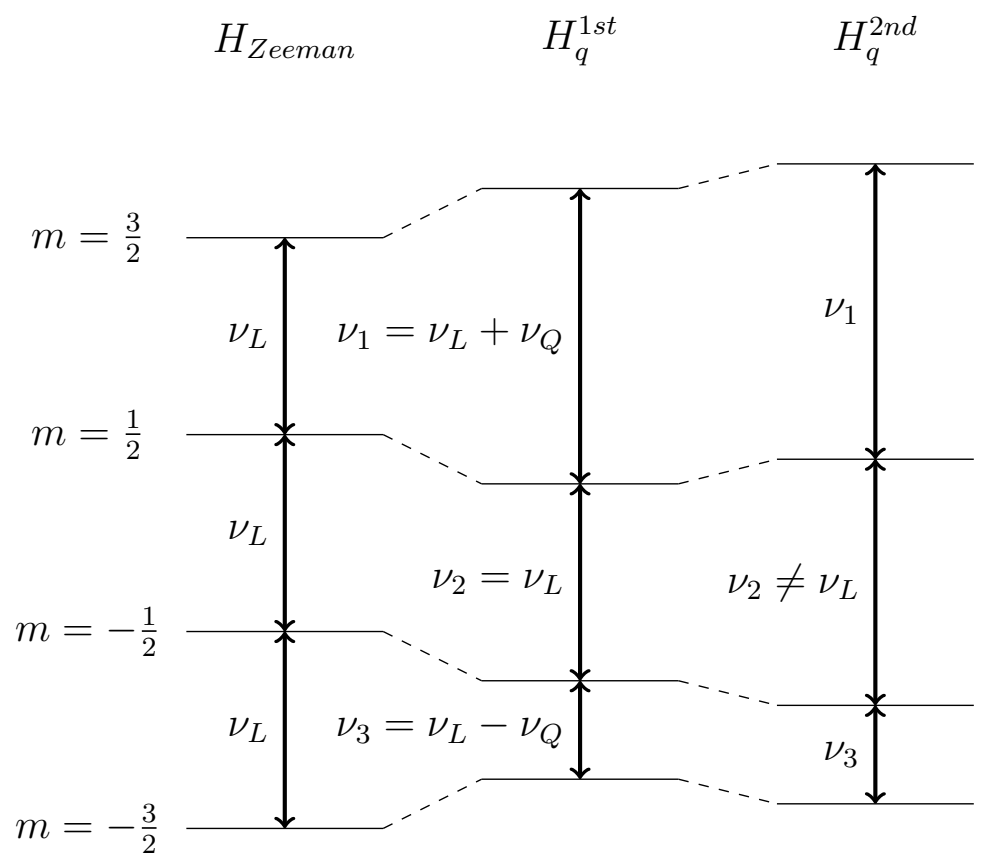

Figure 2.5: NMR energy levels of ${ }^{75} \mathrm{As}$.

The magnetic frequency shift $(\omega=0)$ and relaxation times $\left(\omega=2 \pi f_{\mathrm{NMR}}\right)$ probe the dynamic spin susceptibility, $\chi_{\mathrm{S}}(\omega, q)$, which contains information about the microscopic magnetic structure [112, 113]. Different information can be extracted from various measurements. For example, the simple appearance of additional lines in the spectra offers information about magnetic ordering of the sample. The temperature dependence of the Knight shift (probing the temperature dependence of $\chi_{\mathrm{S}}$ ) can be related to the magnetic ordering. The spin-lattice relaxation time, $T_{1}$, probes spin dynamics, which allows to investigate the increasing spin fluctuations with decreasing temperature. 


\subsection{1 $\quad{ }^{75}$ As NMR shift}

To be able to calculate the shift of the central transition $(m=1 / 2 \rightarrow m=$ $-1 / 2$ ), we need to make to several assumptions. First, we have to assume that the quadrupole interaction can be treated as a perturbation of second order 5 . The second assumption is on the axial symmetry of the electric field gradient (EFG), described by the asymmetry parameter, $\eta$ :

$$
\eta=\frac{V_{x x}-V_{y y}}{V_{z z}}
$$

where $V_{x x}, V_{y y}$ and $V_{z z}$ are the diagonal elements of the EFG ${ }^{6}$ in its principal axis. For the relevant iron-based superconductor studied in this thesis (Ce1111) it is been observed that there is no anisotropy in-plane $V_{x x}=V_{y y}$, which means $\eta=0$. Then the ${ }^{75}$ As NMR frequency of the central transition in a high magnetic field can be written as:

$$
f={ }^{75} \gamma B_{0}(1+K)+\Delta f_{\mathrm{q}}\left(\Theta, B_{0}\right)
$$

where ${ }^{75} \gamma=4.596163 \times 10^{-7} \mathrm{rad} \mathrm{T}^{-1} \mathrm{~s}^{-1}$ is the gyromagnetic ratio of ${ }^{75} \mathrm{As}$, $K$ is the NMR shift and $\Delta f_{\mathrm{q}}$ is the second order quadrupole contribution as a function of the applied magnetic field, $B_{0}$, and $\Theta$ is the angle between $B_{0}$ and the c-axis. Following from equation 2.15 the frequency for $c \| B_{0}$ and $a b \| B_{0}$ is then:

$$
\begin{array}{r}
f_{\|}=\gamma B_{0}\left(1+K_{\|}\right) \\
f_{\perp}={ }^{75} \gamma B_{0}\left(1+K_{\perp}\right)+\frac{3 f_{\mathrm{q}}^{2}}{16 \cdot{ }^{75} \gamma B_{0}}
\end{array}
$$

where the quadrupole frequency measured in $c \| B_{0}$ direction is $f_{\mathrm{q}}$. To be able to calculate the shift ${ }^{75} \mathrm{~K}$ from equations 2.16 and 2.17 , it is necessary to determine the value of ${ }^{75} \gamma B_{0}$. This is done by measuring a reference

\footnotetext{
${ }^{5}$ the first order perturbation lifts the energy degeneracy of the energy levels. It leads to a upward shift of the energy levels of $|m|=3 / 2$ and a downward shift of these with $|m|=1 / 2$ creating three distinct energy differences and hence 3 different resonance frequencies as shown in figure 2.5 .

${ }^{6}$ The EFG is a second-rank symmetrical tensor
} 
compound, which is $\mathrm{NaAsF}_{6}$ as described by Harris et. al. [114]. The NMR shift $K$ usually consists of three different contributions,

$$
K_{\|, \perp}(T)=K_{\mathrm{L}, \|, \perp}+K_{\mathrm{S}, \|, \perp}(T)+K_{\mathrm{M}, \|, \perp}
$$

where $K_{\mathrm{L}}$ is the orbital shift, $K_{\mathrm{S}}$ is the spin (Knight) shift and $K_{\mathrm{M}}$ is the Meissner (diamagnetic) shift. The Meissner shift only appears in superconducting samples below $T_{\mathrm{C}}$ and is small in comparison to the orbital and the Knight shifts observed in our samples, hence $K_{\mathrm{M}}$ can be safely neglected. $K_{\mathrm{L}}$ and $K_{\mathrm{S}}$ are both field independent and magnetic in origin. They are also orientation dependent due to the orientation dependent hyperfine coupling constant. However, only $K_{\mathrm{S}}$ is dependent on temperature, because $K_{\mathrm{S}}$ can be written as $K_{\mathrm{S}}=A_{\|, \perp} \chi_{\mathrm{S}} . A$ is the hyperfine coupling constant and $\chi_{\mathrm{S}}$ the uniform electronic spin susceptibility. Accordingly, only $K_{\mathrm{S}}(T)$ reflects the temperature dependence of $\chi_{\mathrm{S}}(T)$.

\subsubsection{The spin-lattice relaxation time, $T_{1}$ for ${ }^{75} \mathrm{As}$}

The spin-lattice relaxation time, $T_{1}$, was measured with an inversion recovery method, $\tau_{\pi}-t-\tau_{\pi / 2}-\tau_{\text {echo }}-\tau_{\pi}$, where $\tau_{\pi}$ is the time for the $\pi$-pulse which is twice as long as the $\pi / 2$-pulse, $\tau_{\pi / 2}$. The $\pi / 2$-pulse was of the order of several $\mu s$ and a second $\pi$-pulse (the echo) was done after waiting a certain time, $\tau_{\text {echo }}(30 \mu \mathrm{s}$ for the NMR results reported in this thesis). The duration, $t$, between the initial $\pi$-pulse (the inversion) and the rest of the sequence (standard Hahn-echo sequence) was adjusted accordingly. For ${ }^{75} \mathrm{As}$, a nucleus with $I=3 / 2$, the theoretical formula to fit the central transition of a magnetization recovery signal is given by:

$$
M(t)=M(\infty)\left[1-f\left(0.1 \exp \left(\frac{-t}{T_{1}}\right)+0.9 \exp \left(\frac{-6 t}{T_{1}}\right)\right)\right]
$$

where $f$ is a measure of the quality of the inversion with $f=2$ for a perfect inversion. 


\subsubsection{The spin-spin relaxation time, $T_{2}$ for ${ }^{75} \mathrm{As}$}

The spin-spin relaxation time, $T_{2}$, was measured with a normal echo sequence $\tau_{\pi / 2}-\tau_{\text {echo }}-\tau_{\pi}$, where $\tau_{\text {echo }}$ was changed. The obtained signal can then be fitted to either a Lorentzian (equation 2.20) or Gaussian (equation 2.21) decay:

$$
\begin{aligned}
& M(t)=M(0) \exp \left(\frac{-t}{T_{2}}\right) \\
& M(t)=M(0) \exp \left(\frac{-t^{2}}{T_{2}^{2}}\right)
\end{aligned}
$$

where the separation time, $t$, is the time between the initial $\tau_{\pi / 2}$ pulse and the signal, which means $t=2 \cdot \tau_{\text {echo }}$. 


\section{Chapter 3}

\section{Experimental details}

This chapter describes the common sample preparation methods and the measurement techniques starting with the sample characterization throughout the synthesis process to ascertain the sample quality or the changing oxygen content. The electronic and magnetic properties were measured on these samples using a Physical Property Measurement System (PPMS) or a Magnetic Property Measurement Systems (MPMS) from Quantum Design. Nuclear Magnetic Resonance (NMR) experimental details are also discussed including the NMR spectrometer and the NMR magnets. Measurements uncertainties are given in brackets with starting with the last digit, f.e.: $0.38(4)$ meaning that the measured value of 0.38 has a uncertainty of 0.04 and 1350(240) where the value of 1350 has a uncertainty of 240. 


\subsection{Sample preparation}

\subsubsection{Cuprate superconductors}

Samples of $\mathrm{FeSr}_{2} \mathrm{YCu}_{2} \mathrm{O}_{6+\mathrm{y}}(\mathrm{Fe} 1212)$ and $\mathrm{FeSr}_{2} \mathrm{Y}_{2-\mathrm{x}} \mathrm{Ce}_{\mathrm{x}} \mathrm{Cu}_{2} \mathrm{O}_{8+\mathrm{y}}(\mathrm{Fe} 1222)$ were made from powders of $\mathrm{Fe}_{2} \mathrm{O}_{3}, \mathrm{Sr}\left(\mathrm{NO}_{3}\right)_{2}, \mathrm{Y}_{2} \mathrm{O}_{3}, \mathrm{CuO}$ and $\mathrm{Ce}_{2} \mathrm{O}_{3}$ (for Fe1222). Some samples of Fe1212 were also made by adding $\mathrm{ZnO}$ so that $\mathrm{Zn}$ partially substitutes on the copper site, while adding $\mathrm{CaO}$ or $\mathrm{La}_{2} \mathrm{O}_{3}$ for hole (Ca) and electron (La) doping. These powders are ground together and then denitrated on a gold foil. Since $\operatorname{Sr}\left(\mathrm{NO}_{3}\right)_{2}$ was used as a starting product, the nitrogen had to be removed. The target material itself was synthesized in the second step in air at temperatures above $1000^{\circ} \mathrm{C}$. Further steps involved annealing in a nitrogen or an argon atmosphere to redistribute as much of the Fe as possible from the $\mathrm{Cu}(2)$ plane to the $\mathrm{Cu}(1)$ chain site with subsequent quenching in liquid nitrogen. Those samples were annealed in an oxygen atmosphere to increase their oxygen content to promote the appearance of superconductivity. The high temperature furnaces used are shown in figure 3.1 . 


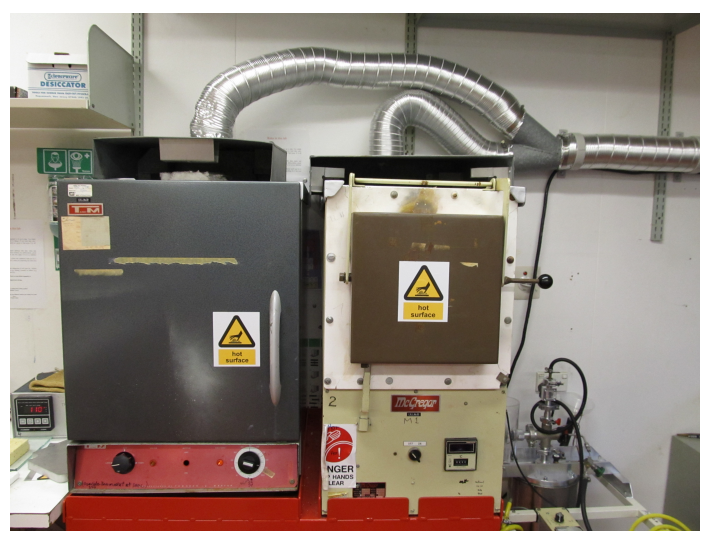

(a) Ventilated furnace for denitration.

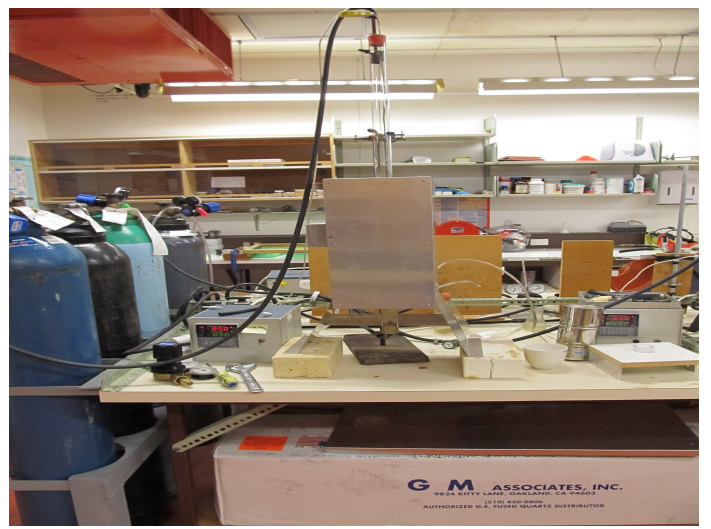

(b) Vertical furnace for argon treatment and quenching.

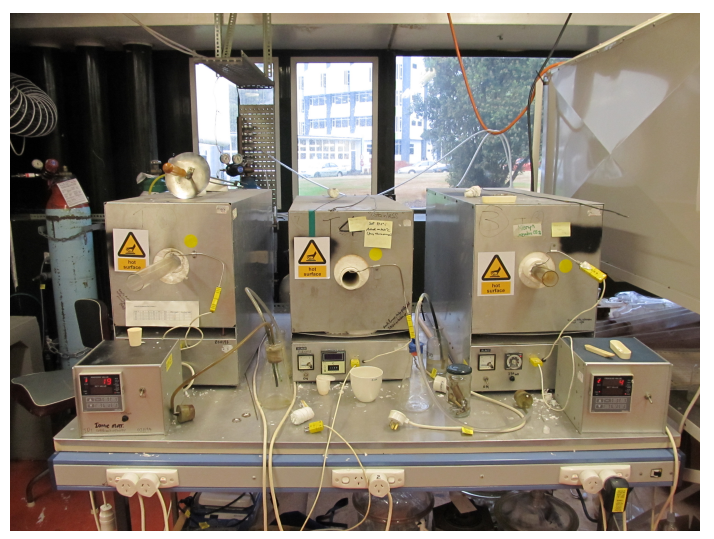

(c) Horizontal furnace for initial synthesis and oxygen loading.

Figure 3.1: High temperature furnaces used for synthesis of the iron-cuprate hybrids. 


\subsubsection{Iron-based superconductor}

The preparation of pnictide samples is significantly more difficult than the preparation of the cuprates, because the starting materials as well as the reaction products are sensitive to oxygen and water contamination, and the central ingredient is arsenic $\left(\mathrm{A}^{1}\right)$. Hence, the preparation has to be done in the controlled environment of a glove box (figure 3.2a) where the humidity and oxygen content were kept very low (below 3ppm for oxygen and there are two water traps to keep the water content at a similar level). The glove box also ensures the arsenic is handled safely.

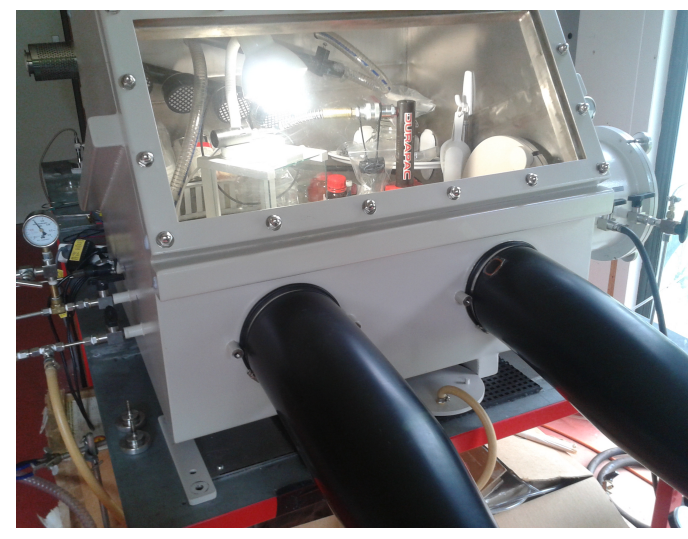

(a) Humidity and oxygen controlled glove box.

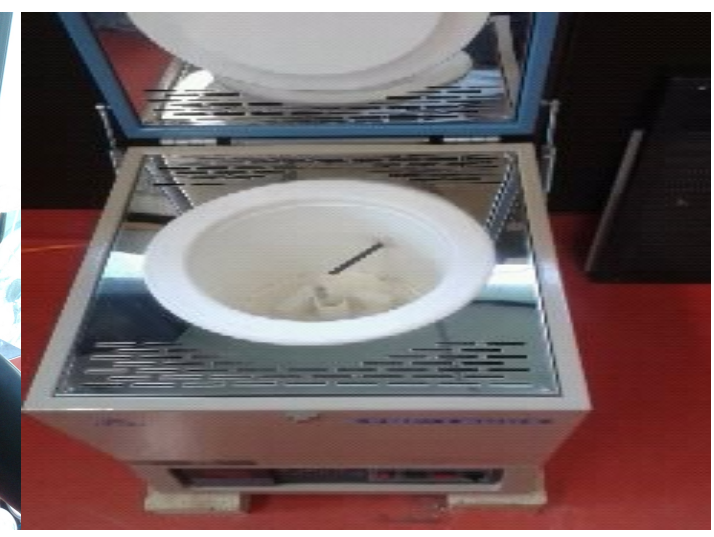

(b) High temperature oven (up to $1250^{\circ} \mathrm{C}$ ) for iron-based superconductor synthesis.

Figure 3.2: Equipment used in the synthesis of iron-based superconductors.

The starting materials were mixed inside the glove box and encapsulated in double walled sealed quartz tubes (figure 3.3) to ensure that no oxygen could reach the material through diffusion and that no arsenic could escape. The quartz tubes were filled inside the glove box which contains high purity argon (99.998\%). They were then taken outside where the open end was sealed by a vacuum valve. The Tubes were then evacuated and sealed by heating and melting the quartz with a glass-blower torch. The samples in the quartz tubes were heated up to temperatures of $1500 \mathrm{~K}$ (in a high temperature

\footnotetext{
${ }^{1}$ Arsenic is very toxic, for example: the estimated lethal dose of inorganic arsenic in drinking water is $1 \mathrm{mg} \mathrm{kg}^{-1}$ per day.
} 
oven shown in figure $3.2 \mathrm{~b}$ depending on the material being synthesized. The final samples were stored in an oxygen and water free environment.

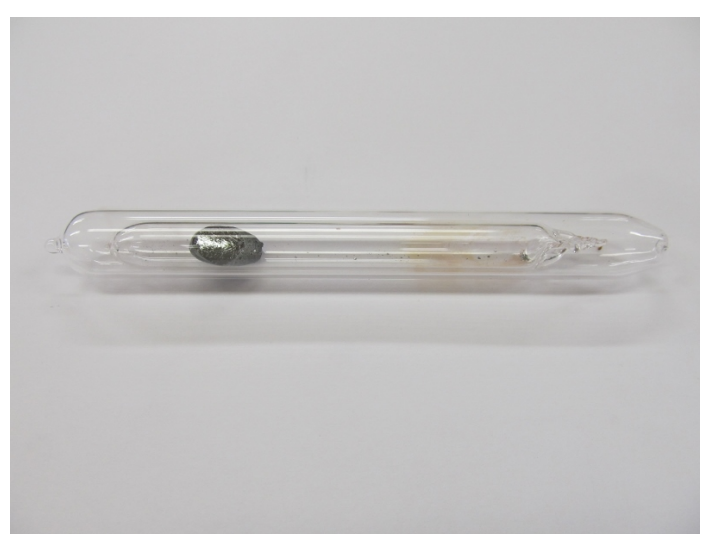

Figure 3.3: An example of a double walled quartz tube sealed under reduced pressure with a $\mathrm{SrFe}_{2} \mathrm{As}_{2}$ sample surrounded by flux. 


\subsection{Sample characterization}

The samples where characterized after each step in the synthesis by powder X-ray diffraction (XRD). The room-temperature thermopower, $S(300 \mathrm{~K})$, was measured for later steps to get an idea of the oxygen content using a $S(300 \mathrm{~K})$ versus hole concentration correlation [115]. Some of the iron pnictide samples (for example,. $\mathrm{BaFe}_{2} \mathrm{As}_{2}$ ) were later viewed in a scanning electron microscope (SEM) to investigate their surface and composition. Raman spectroscopy was performed on the $\mathrm{FeSr}_{2} \mathrm{YCu}_{2} \mathrm{O}_{6+\mathrm{y}}$ system to investigate possible changes to the Raman modes with hole doping.

\subsubsection{X-ray diffraction}

The X-ray diffraction measurements were done on pressed powder pellets using a Philips PW1700 series powder diffractometer shown in figure 3.4 . It has a Co tube $(\lambda=1.789 \AA)$ with an operating voltage of $40 \mathrm{kV}$ and a current of $20 \mathrm{~mA}$. The obtained XRD data were analyzed by EVA software to determine the sample quality.

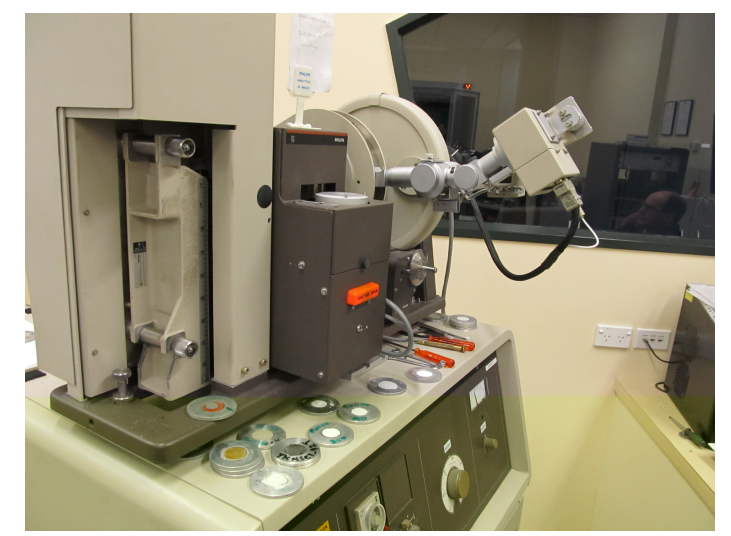

Figure 3.4: Philips PW1700 series powder diffractometer. 


\subsubsection{Home built thermopower setup}

The room temperature thermopower, $S(300 \mathrm{~K})$, was determined using a home built thermopower setup shown in figure 3.5. The sample pellet was squeezed in between two copper plates, which are used to measure the voltage across the sample. A simple resistor is used to heat one side of the sample to create a temperature gradient across the sample and a differential thermocouple measured the change in temperature. The thermoelectric voltages were measured before and after a stable temperature gradient was applied. The thermopower was obtained from $\Delta V / \Delta T$ were $\Delta V$ is the change in voltage and $\Delta T$ is the change in temperature.

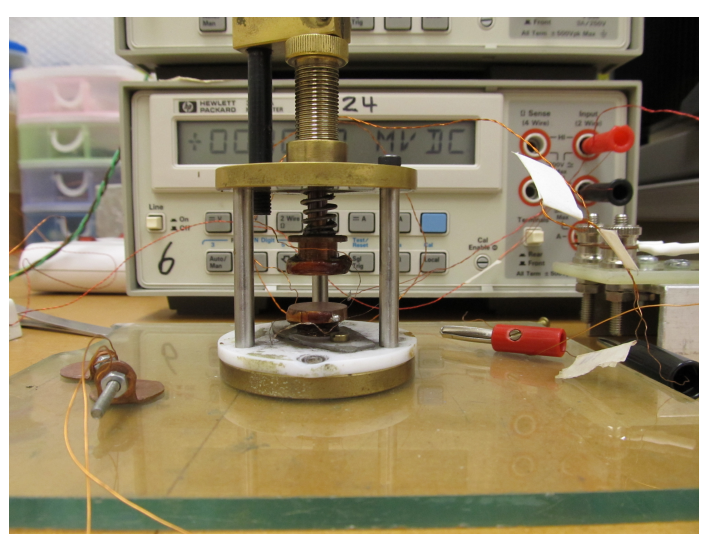

Figure 3.5: Room temperature thermopower measurement setup. 


\subsubsection{Scanning electron microscope}

A Quanta scanning electron microscope (SEM) (figure 3.6) was used for imaging the iron pnictide samples. Several different configurations were used including bright-field, dark-field imaging and electron back scattering to obtain the images. Energy dispersive X-ray spectroscopy (EDS) measurements were done to ascertain the composition and sample quality.

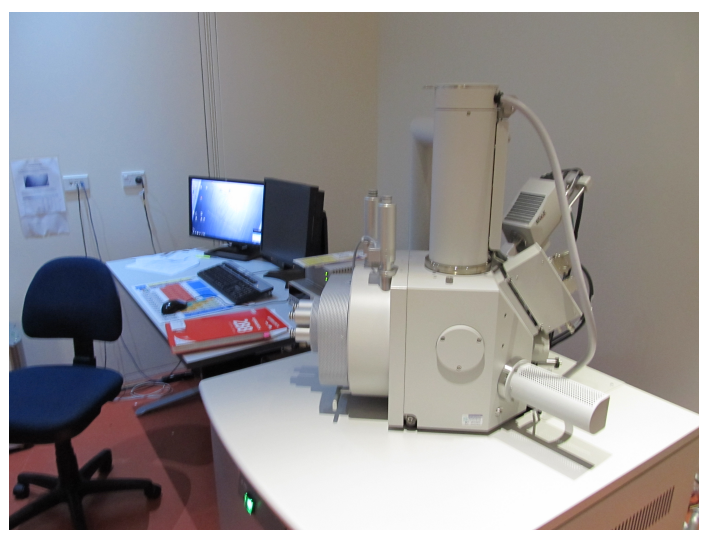

Figure 3.6: Quanta SEM 


\subsubsection{Raman spectroscopy}

The Raman measurements were carried out using a $514 \mathrm{~nm}$ green Ar-ion laser on a LabRAM system shown in figure 3.7. The LabRAM setup uses a continuously adjustable confocal aperture in the object plane of the microscope with an Olympus BX objective enabling high performance in spatial and axial resolution. A laser notch filter was used to reject the laser line. There is a single grating to disperse the scattered light and it is possible to select a 600 lines per mm or an 1800 lines per mm grating using a translating shaft to provide different spectral resolutions. The dispersed light was detected using a cooled highly sensitive $\mathrm{CCD}^{2}$ linear array detection system and laser notch filter technology is used inside the single stage spectrometer. The system also provides the possibility to maximize sensitivity at different excitation laser lines by selecting specifically blaze optimized gratings.

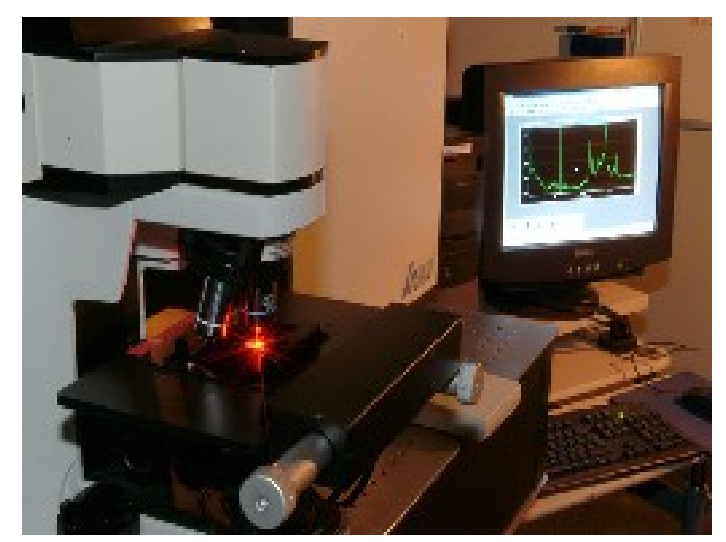

Figure 3.7: LabRAM

\footnotetext{
${ }^{2} \mathrm{CCD}=$ charge-couple-device
} 


\subsection{The Physical Property Measurement Sys- tems and the Magnetic Property Mea- surement System}

A large part of the investigation of the sample properties were done using the commercial Physical Property Measurement System (PPMS) and Magnetic Property Measurement System (MPMS) system from Quantum Design (figure 3.8). Detailed information about the equipment can be found on their website.

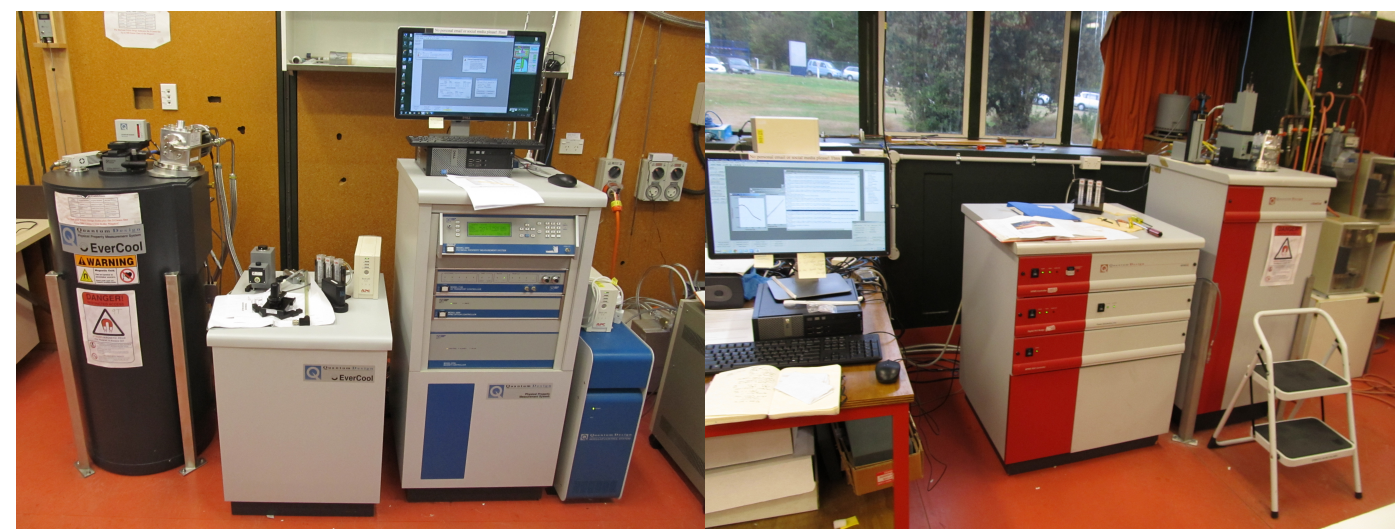

(a) PPMS

(b) MPMS

Figure 3.8: Magnetic lab at Robinson Research Institute (RRI) with a) PPMS and b) MPMS.

\subsubsection{Resistivity and magnetoresistance}

Resistivity measurements were performed with the resistivity option of the PPMS, which includes a sample rotator to orient the sample relative to the applied magnetic field for magnetoresistance (MR) measurements. A 4-point probe configuration was used and the sample contacts were established with a two-part silver epoxy from EPO-TEK. An example can be seen in figure 3.9. where a sample of $\mathrm{BaFe}_{2} \mathrm{As}_{2}$ is shown with copper wire contacts on it. Similar configurations were used for all samples and the resultant resistivity, $\rho$, can be calculated from the measured resistance $R$, the cross-section area 
$A$, and the voltage contacts separation $l$ :

$$
\rho=\frac{R \cdot l}{A}
$$

The cross-section area is determined by the thickness of the sample typical in the order of $0.1 \mathrm{~mm}$ and the relevant width between the two voltage contacts (inner two) typically around $0.5 \mathrm{~mm}$. However, the uncertainty in the resistivity is rather large for the iron-arsendide samples due to their size and hence the width of the sample is usually larger than the voltage separation. Therefore, the resistance is usually displayed for those samples. This is also no problem for the analysis, because the values are consistent in each other (for example figure 4.2 and 4.3) and there are only trends analyzed, where it is not important if that absolute value of the resistivity is 5 times bigger or smaller.

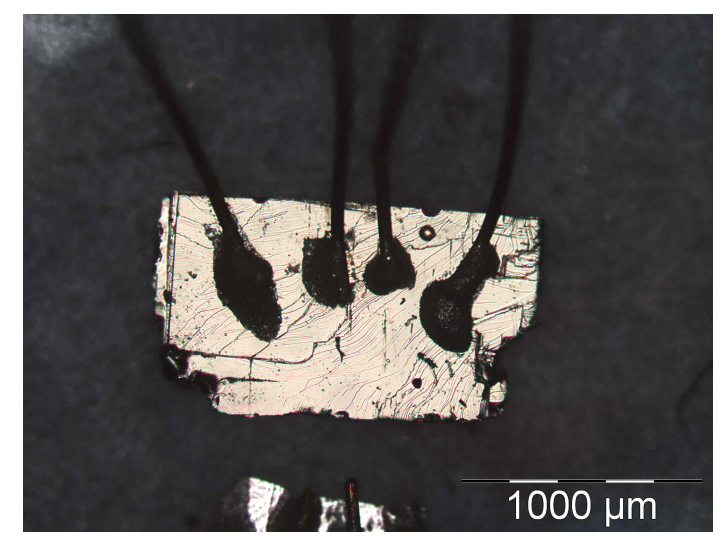

Figure 3.9: A sample of $\mathrm{BaFe}_{2} \mathrm{As}_{2}$ with copper wires connected for resistivity and magnetoresistance measurements. 


\subsubsection{DC magnetization in a vibrating sample magne- tometer}

DC magnetization measurements were performed using the vibrating sample magnetometer (VSM) option of the PPMS. A small portion of the sample weighing around $10 \mathrm{mg}$ to $50 \mathrm{mg}$ was placed in a gelatin capsule mounted on a brass holder (figure 3.10), which was inserted into the VSM.

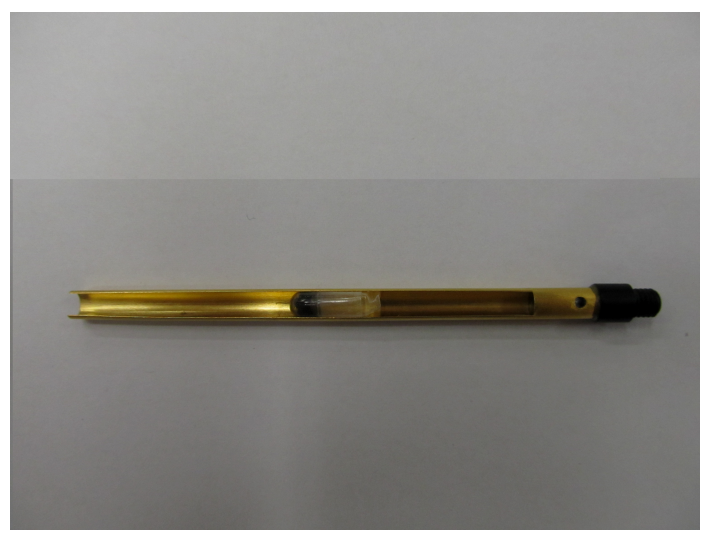

Figure 3.10: Sample in the gelatin capsule in a brass sample holder for the VSM option of the PPMS.

The vibrating sample magnetometer (VSM) was initially invented by Simon Foner in 1956 [116] and is nowadays available as a dedicated option of the PPMS. The PPMS provides a static and uniform magnetic field to magnetize the sample, which is physically vibrated sinusoidally inside this field. This movement induces a voltage in the surrounding pickup coils which is proportional to the magnetic moment of the sample. Hence, the obtained voltage can be directly related to the magnetic moment of the sample $m^{\mathrm{emu}}$, in [emu], which can subsequently be converted to SI magnetization in $\left[\mathrm{A} \mathrm{m}^{-1}\right]$ as shown below,

$$
M=\frac{m^{e m u}}{m} \rho \cdot 1000
$$

where $\rho$ is the density of the sample in $\left[\mathrm{g} / \mathrm{cm}^{3}\right]$ and $m$ the mass of the sample in $[\mathrm{g}]$.

The susceptibility, $\chi$, in the paramagnetic state can be written in the 
Curie-Weiss (CW) form as,

$$
\chi=\frac{M}{H}=\frac{N \mu_{0} \mu_{\mathrm{B}}^{2} P_{\mathrm{eff}}^{2}}{3 V k_{\mathrm{B}}(T-\Theta)}+\chi_{0}
$$

where $V$ is the volume that contains $N$ magnetic ions, $\mu_{0}$ is the vacuum permeability, $P_{\text {eff }}$ is the effective moment per magnetic ion $(\mathrm{Fe}$ and $\mathrm{Cu}$ ) in units of Bohr magnetons $\mu_{\mathrm{B}}, \Theta$ is the CW temperature, $T$ is the temperature and $\chi_{0}$ is the temperature independent susceptibility. When fitting the data the Curie constant, $C$, is obtained and can be written as:

$$
C=\frac{N \mu_{0} \mu_{\mathrm{B}}^{2} P_{\mathrm{eff}}^{2}}{3 V k_{\mathrm{B}}}
$$

The effective moment per formula unit in Bohr magnetons can be calculated from $C$ [18]:

$$
P_{\text {eff }}^{\text {f.u. }}=\sqrt{\frac{3 k_{\mathrm{B}} V C}{N \mu_{0} \mu_{\mathrm{B}}^{2}}}
$$

The relevant volume is the volume per formula unit, where the number of iron and copper atoms is known. This volume per formula unit, $V_{\text {f.u. }}$, can be calculated from,

$$
V_{\text {f.u. }}=\frac{M_{r}}{N_{\mathrm{A}} \rho}
$$

where $M_{r}$ is the molar mass and $N_{\mathrm{A}}$ is Avogadro's constant. The susceptibility will depart from the $\mathrm{CW}$ temperature dependence as the temperature approaches a magnetic ordering transition. This departure can be seen by a plot of $1 /\left(\chi-\chi_{0}\right)$ against temperature, which is linear in the paramagnetic temperature region. The value for the Curie-constant is represented by the slope of the linear region and the Curie-temperature by the intercept with the temperature-axis. 


\subsubsection{DC magnetization with the MPMS}

An alternative to the VSM for DC magnetization measurements is the standard transport of the MPMS. Each sample was mounted in a plastic straw and then inserted into the standard transport of the MPMS. The moment is measured by moving the sample through a series of coils that are connected to a SQUID, which is the acronym for superconducting quantum interference device. The SQUID, consisting of a superconducting loop containing a Josephson junction [117, 118], is a very sensitive magnetometer and moments below 1 pemu can be measured.

\subsubsection{AC magnetization}

AC magnetization measurements were performed with the ACMS option of the PPMS to determine the frequency dependence of magnetic transitions. The ACMS has an AC drive coil that provides an AC magnetic field. A DC magnetic field can also be applied using the superconducting magnet. It is then possible to measure the sample response under a small alternating excitation field provided by the AC drive coil. The AC moment can be measured from $10 \mathrm{~Hz}$ to $10000 \mathrm{~Hz}$. 


\subsection{Nuclear Magnetic Resonance setup}

NMR measurements were done at the University of Leipzig in the group of Prof. Jürgen Haase, where the necessary equipment is available. A magnet (figure 3.11a) with a field of $11.74 \mathrm{~T}$ was used for NMR measurements, which corresponds to a proton frequency of $500 \mathrm{MHz}$. To check the field dependence of the NMR signal smaller and larger magnets with fields of $7.04 \mathrm{~T}$ and $17.63 \mathrm{~T}$ were also used. These magnets supplied an uniform static external field, $B_{0}$, which leads to a Zeeman splitting. The samples were mounted in a tuneable and matchable probehead (figure 3.11c), and placed inside a continuous flow cryostat allowing NMR measurements to be performed in a temperature range of 300 to $5 \mathrm{~K}$. Two different spectrometers were used - the first is from Apollo (figure 3.11b and the second from Bruker. Both provide high power radio frequency pulses and can detect the resultant NMR signal. 


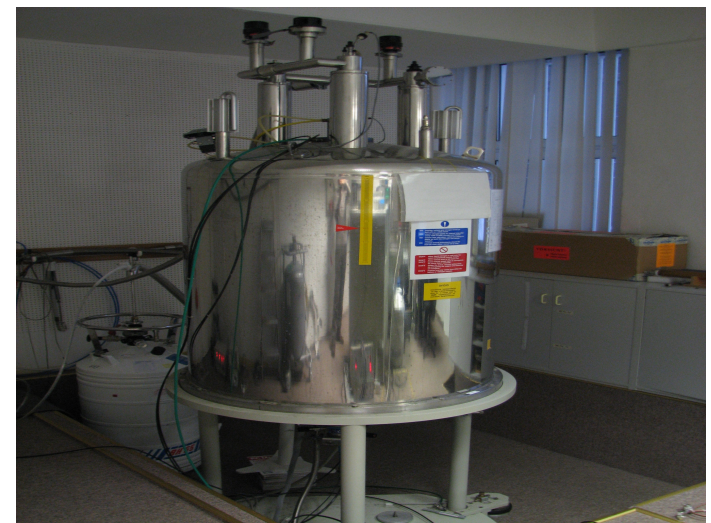

(a) $11.74 \mathrm{~T}$ magnet corresponding to a proton NMR frequency of $500 \mathrm{MHz}$

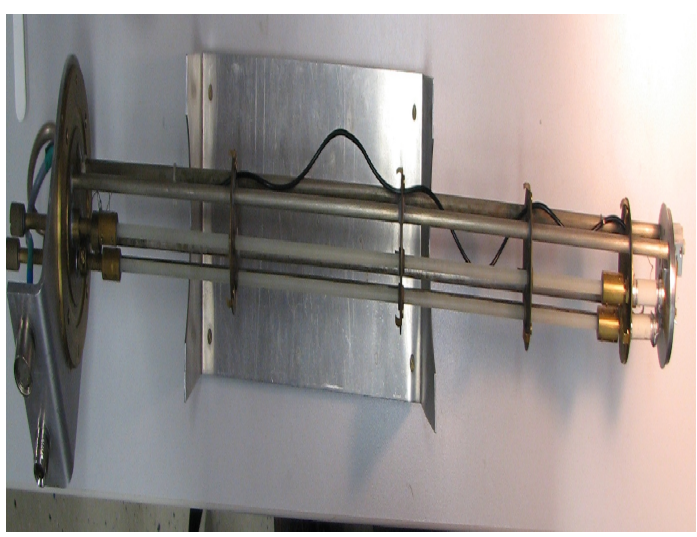

(c) Tuneable and matchable NMR probehead 


\subsubsection{The resonant circuit}

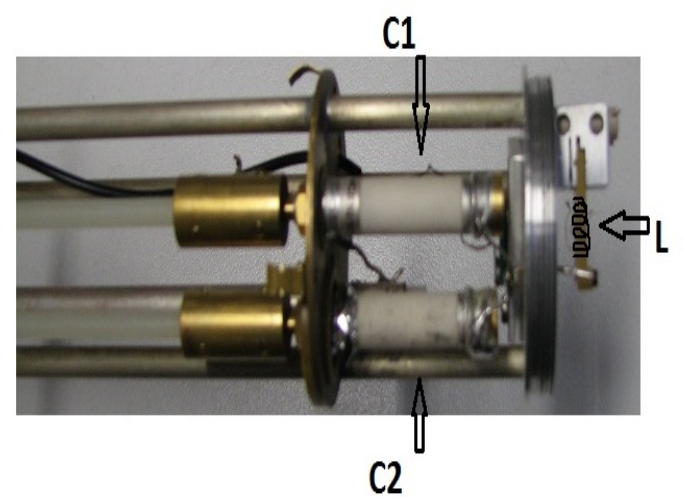

(a) Enlargement of the NMR probehead.

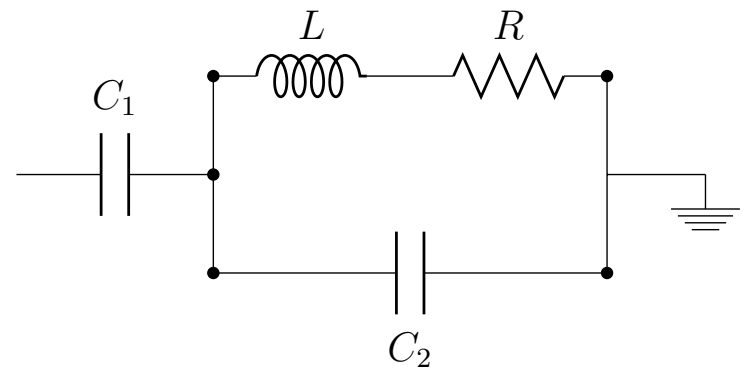

(b) Schematic of the resonant circuit

Figure 3.12: NMR probehead with resonant circuit.

The probehead itself contains a capacitive loaded resonant circuit, which is shown in figure 3.12. The actual resonant circuit has an additional capacitor, $C_{1}$, placed before it to match the input impedance of the spectrometer of $50 \Omega$. The circuit then contains a second capacitor, $C_{2}$, to tune the resonant circuit to the desired resonance frequency. The resistance of the circuit comes nearly completely from the coil (with the inductance $L$ ), which is indicated by a resistor in series with the coil. Then the frequency, $f_{0}$, of the resonant circuit is given by

$$
f_{0}=\frac{1}{2 \pi \sqrt{L\left(C_{1}+C_{2}\right)}}
$$




\section{Chapter 4}

\section{Magnetic and electronic properties of $\mathrm{CeFeAsO} \mathrm{O}_{1-\mathrm{x}} \mathrm{F}_{\mathrm{X}}$}

\subsection{Introduction}

Superconductivity in the iron-based superconductors with higher superconducting transition temperatures, $T_{\mathrm{C}}$, was first discovered in $\mathrm{LaFeAsO}_{1-\mathrm{x}} \mathrm{F}_{\mathrm{x}}$ (La1111) [29] with a maximum $T_{\mathrm{C}}$ of $26 \mathrm{~K}$. La[119] can be replaced with other rare-earth ions (R-1111) such as Ce [2, 120], Pr [121], Nd [57], Sm [122] and others [6, 123, 124]. The highest recorded $T_{\mathrm{C}}$ is $56 \mathrm{~K}$ [30]. When $\mathrm{La}$ is replaced by $\mathrm{Ce}$ (in $\mathrm{CeFeAsO}_{1-\mathrm{x}} \mathrm{F}_{\mathrm{x}}$ ) superconductivity starts to appear at a fluoride $(\mathrm{F})$ concentration of $\mathrm{x}=0.06$, reaching a plateau in $T_{\mathrm{C}}=41 \mathrm{~K}$ around $\mathrm{CeFeAsO}_{0.84} \mathrm{~F}_{0.16}$ [2, 10, 125] and slowly increasing further even beyond $\mathrm{x}=0.30$ [9]. Hence, the superconducting dome is rather flat when compared to the cuprates. For a fluoride content below $\mathrm{x}=0.06$ there is a spin-density-wave (SDW) transition [2], which is associated with an antiferromagnetic order of the Fe moments. The SDW transition occurs at a temperature of $140 \mathrm{~K}$ [2] for the parent compound of Ce1111. The SDW transition temperature is decreasing with increasing $\mathrm{F}$ doping and vanishes when superconductivity appears. There is also a structural transition from tetrahedral to orthorhombic around a temperature of $155 \mathrm{~K}$ for pure Ce1111 [2]. The structural transition temperature decreases with increasing F con- 
tent up to $\mathrm{x}=0.10$, where it vanishes into the superconducting dome [2]. The Ce moments order below $5 \mathrm{~K}$ [2] for the pure Ce1111 material and the ordering temperature decreases with increasing $\mathrm{F}$ doping.

In addition to having a high $T_{\mathrm{C}}$ the 1111-family also exhibits a very high upper critical field, $B_{\mathrm{c} 2}$, and a high irreversible field, $B_{\text {irr }}$ [126 128]. Both are very important properties for practical applications and the latter suggests a higher superconducting critical current density. Earlier studies on superconducting $\mathrm{CeFeAsO}_{0.9} \mathrm{~F}_{0.1}$ samples indicate $B_{\mathrm{c} 2}$ values between $94 \mathrm{~T}$ [129] to $185 \mathrm{~T}$ [130]. 


\subsection{Synthesis and structure}

Polycrystalline samples of $\mathrm{CeFeAsO}_{1-\mathrm{x}} \mathrm{F}_{\mathrm{x}}$ were synthesized using a standard solid state reaction method, where FeAs and CeAs were pre-synthesized [131] and ground together with $\mathrm{Fe}, \mathrm{As}, \mathrm{CeO}_{2}$ and $\mathrm{CeF}_{3}$. The powder was pressed into pellets and sealed into evacuated quartz tubes. These pellets were then heated to $1000^{\circ} \mathrm{C}$ for $24 \mathrm{~h}$ before a second heating to $1180{ }^{\circ} \mathrm{C}$ for $50 \mathrm{~h}$ with an intermediate grinding and repelleting. The quality of all the prepared samples were checked by powder XRD measurements (figure A.1), which showed minor impurities caused by FeAs, $\mathrm{FeAs}_{2}$ and $\mathrm{Ce}_{3} \mathrm{Fe}_{4} \mathrm{O}_{3}$. Superconductivity was confirmed in the higher doped samples of $\mathrm{x} \geq 0.13$ by resistivity measurements. For NMR measurements all samples were mixed with a slow curing epoxy (EPO-TEK 301) and aligned in a $1 \mathrm{~T}$ magnet rotating at $60 \mathrm{rpm}$ with the field perpendicular to the rotating axis. XRD analysis of the aligned samples via rocking curves ${ }^{1}$ showed the (004) reflection's full width at half maximum (FWHM) of around $7^{\circ}$ or smaller employing $\mathrm{Co}-\mathrm{K}_{\alpha}$ radiation.

$\mathrm{CeFeAsO}_{1-\mathrm{x}} \mathrm{F}_{\mathrm{x}}$ has a layered structure of alternating $\mathrm{CeO}$ and $\mathrm{FeAs}$ layers (figure 4.1). There are two important facts worth mentioning here: the 1111 family is closest to being 2D-like similar to the cuprates, and secondly, the $\mathrm{Fe}$ and $\mathrm{As}$ are not planar (unlike $\mathrm{Cu}$ and $\mathrm{O}$ in the cuprates), but sit in a tetrahedral configuration, where arsenic atoms are above and below the iron. This appears to be important as the highest values for $T_{\mathrm{C}}$ are observed for the doping where the Fe-As configuration approaches a perfect tetrahedron [2].

\footnotetext{
${ }^{1}$ fixed $2 \Theta$ angle on a certain strong peak to determine the quality of the alignment, which is important for NMR measurements
} 


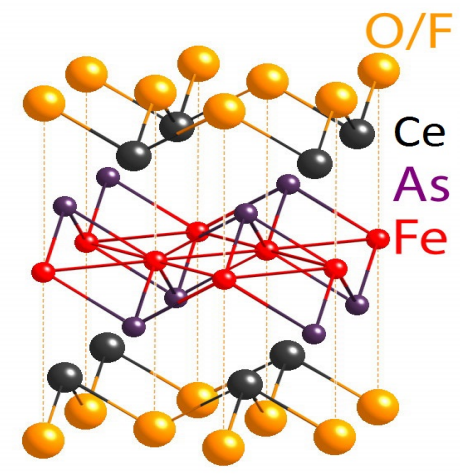

Figure 4.1: Crystal structure of $\mathrm{CeFeAsO}_{1-\mathrm{x}} \mathrm{F}_{\mathrm{x}}$ (adjusted from [132]). 


\subsection{Resistivity $\rho$}

The resistivity was measured with a 4-point-probe configuration under different applied magnetic fields. All samples show a low resistivity value at room temperature in the $\mathrm{m} \Omega \mathrm{cm}$ range.

\subsubsection{Zero field resistivity of the non-superconducting sample $\mathrm{x}=0.07$}

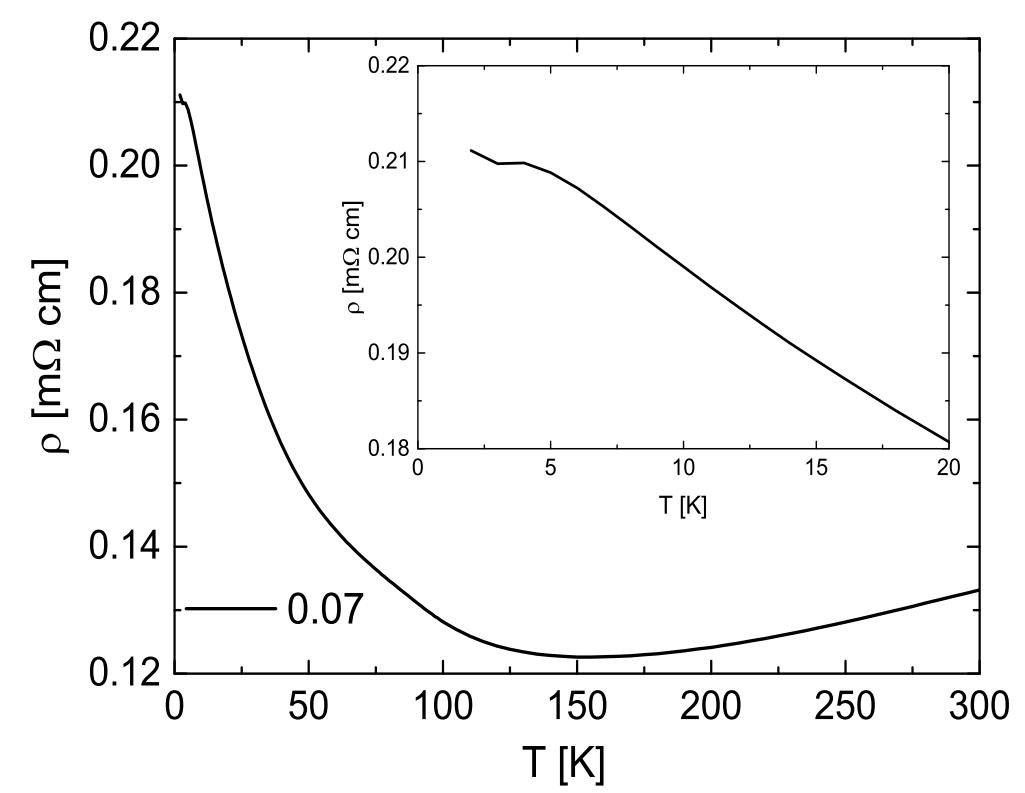

Figure 4.2: Temperature dependence of the resistivity for the 0.07 sample with a minimum around $150 \mathrm{~K}$. Inset: Enlarged low temperature region where there is a small decrease of the resistivity due to antiferromagnetic order of the Ce moments.

Figure 4.2 shows temperature dependent resistivity for $\mathrm{x}=0.07$, which initially decreases with decreasing temperature from $300 \mathrm{~K}$ to a minimum around $150 \mathrm{~K}$ before increasing with temperature. However, neither a SDW transition, which should have resulted in a significant drop in resistivity at the transition temperature, nor a superconducting transition, with the decrease 
to zero resistivity, were observed. Examples of these aforementioned features are seen at different dopings in $\mathrm{CeFeAsO}_{1-\mathrm{x}} \mathrm{F}_{\mathrm{x}}[120$, 133, 134] and in $\mathrm{LaFeAsO}_{1-\mathrm{x}} \mathrm{F}_{\mathrm{x}}[29$, 135]. The sample with $\mathrm{x}=0.07$ is expected to be in the region were SDW ordering vanishes and superconductivity emerges. The observed resistivity in figure 4.2 indicates that the sample is close to that point, where neither SDW nor superconducting ordering take place. It is clearly non-superconducting and shows only a slight indication of a transition (inset of figure 4.2 below $4 \mathrm{~K}$, which is likely to be due to antiferromagnetic ordering of the Ce moments [2]. In the next chapter, NMR measurements (figure 5.15 confirms a SDW transition in the aligned sample of $\mathrm{CeFeAsO}_{0.93} \mathrm{~F}_{0.07}$ around $20 \mathrm{~K}$. The change in slope in the resistivity around $150 \mathrm{~K}$ is too high to be due to Kondo behavior. However, these are polycrystalline powders similar to the cuprates and other polycrystalline samples where such behavior is seen for transport through grain boundaries.

\subsubsection{Zero field resistance for superconducting samples}

The temperature dependence of the resistance is shown in figure 4.3 for all superconducting samples $(\mathrm{x}=0.13,0.15,0.20,0.25) . T_{\mathrm{C}}$ is found to increase from $35(1) \mathrm{K}$ for $\mathrm{x}=0.13$ to $42(1) \mathrm{K}$ for $\mathrm{x}=0.25$ which is consistent with the reported phase diagrams in references [2, 9, 120]. The temperature dependence of the resistivity follows a Fermi-liquid (proportional to $T^{2}$ ) behavior until the vicinity of the superconducting transition, where there are superconducting fluctuations followed by a superconducting transition in which the resistivity drops to zero. This region above $T_{\mathrm{C}}$ can be analyzed in terms of fluctuation conductivity (FC) [136, 137] and the region below $T_{\mathrm{C}}$ can be broadened by thermally assisted motion of the vortices similar to the cuprates [138]. 


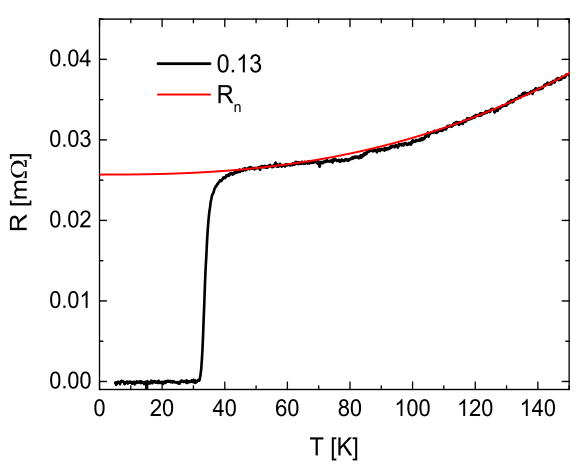

(a) $\mathrm{x}=0.13$

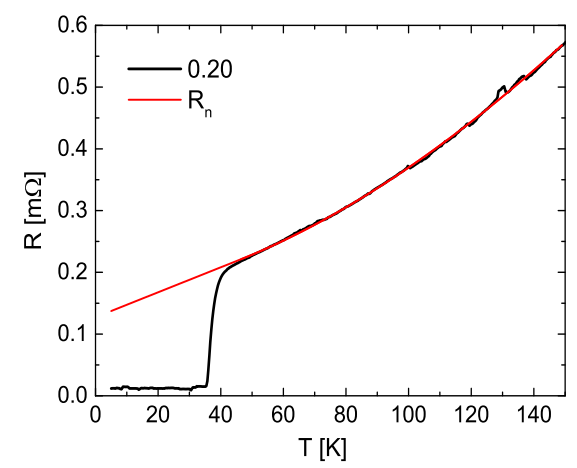

(c) $\mathrm{x}=0.20$

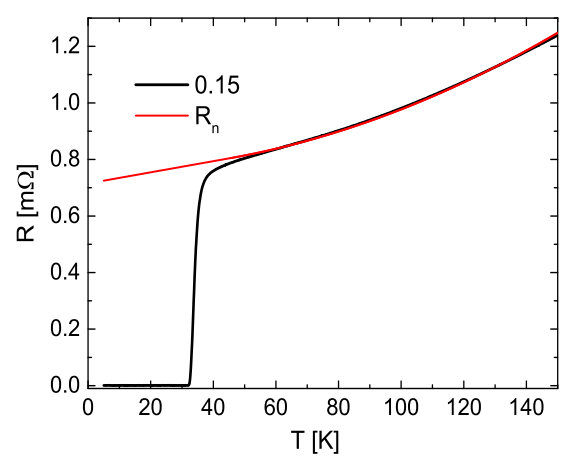

(b) $\mathrm{x}=0.15$

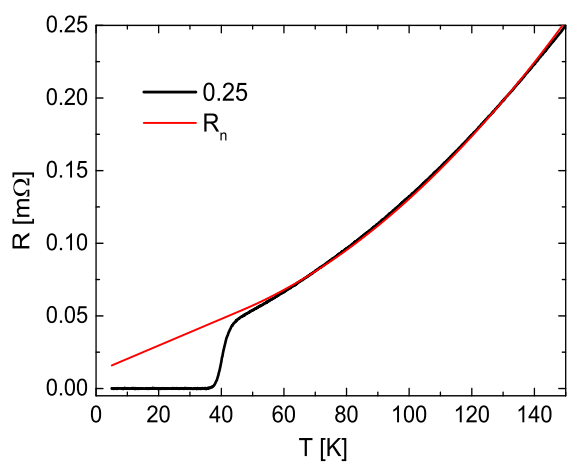

(d) $\mathrm{x}=0.25$

Figure 4.3: Temperature dependence of the resistance for all the superconducting samples with a Fermi liquid fit to the normal state resistance.

\subsubsection{Fluctuation conductivity of superconducting sam- ples}

The fluctuation conductivity becomes noticeable by the deviation of the resistivity from the normal state behavior in the vicinity of the superconducting transition. For layered superconductors there are two dimensional superconducting fluctuations at higher temperatures and three dimensional superconducting fluctuations very close to $T_{\mathrm{C}}$ [136]. The fluctuation conductivity was calculated from the resistivity by subtracting the normal-state resistiv- 
ity from the measured resistivity. The normal-state resistivity is observed to follow a Fermi-liquid temperature dependent behavior,

$$
R_{\mathrm{n}}(T)=R_{0}+A \cdot T^{2}
$$

where $R_{0}$ is the temperature independent resistance. Equation 4.1 is then fitted to the measured resistance above $60 \mathrm{~K}$ and the difference between normalstate resistance and the observed resistance is calculated at the vicinity of the superconducting transition to obtain the excess conductivity, $\Delta \sigma$. The fitted normal-state resistance are shown in figure 4.3 as red lines and the calculated fluctuation conductivity in figure 4.4. The fluctuation conductivity in a layered superconductor is due to an acceleration of superconducting pairs and can be written as [136, 137] in the case of $\epsilon \ll 1$,

$$
\Delta \sigma=\frac{\pi e^{2}}{16 h d \epsilon\left[1+\left(\frac{2 \xi_{c}}{d}\right)^{2}\left(\frac{1}{\epsilon}\right)\right]^{1 / 2}}
$$

where e is the elemental charge, $\mathrm{h}$ is the Planck constant, $\mathrm{d}$ is the separation between the superconducting planes, $\xi_{c}$ is the c-axis coherence length and $\epsilon=T / T_{C}-1$. Figure 4.4 also shows the fit to $3 \mathrm{D}$ regions $\left(\xi_{\mathrm{C}}>d\right)$, where the fluctuation conductivity follows a $\Delta \sigma \propto \epsilon^{-0.5}$ dependence for 3D [136]. There is a crossover between $3 \mathrm{D}$ to $2 \mathrm{D}$ as the temperature is increased and the crossover is closer to $T_{\mathrm{C}}$ for lesser fluoride content, which is due to a smaller coherence length. Liu et. al. [139] investigated $\mathrm{RFeAsO}_{1-\mathrm{x}} \mathrm{F}_{\mathrm{x}}(\mathrm{R}=\mathrm{Nd}$, Pr, Sm) where they also found a small 3D temperature region crossing over to a larger temperature region with 2D fluctuations in agreement with our observations. A similar crossover is also reported for LiFeAs [140]. 


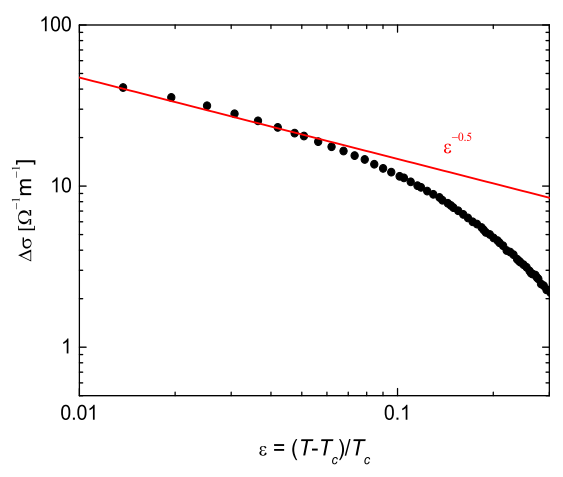

(a) $\mathrm{x}=0.13$

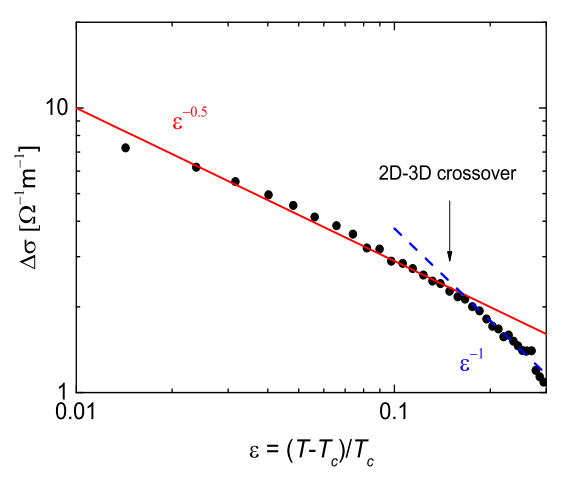

(c) $\mathrm{x}=0.20$

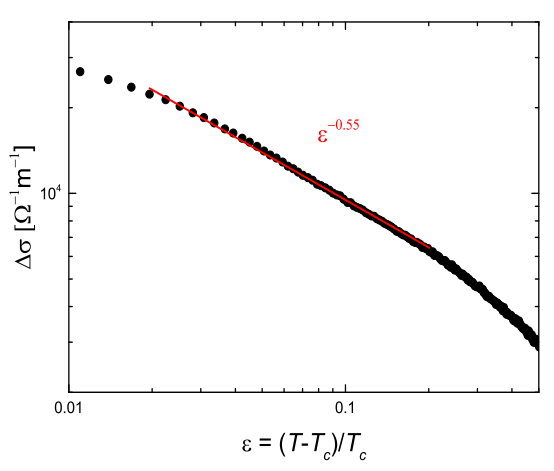

(b) $\mathrm{x}=0.15$

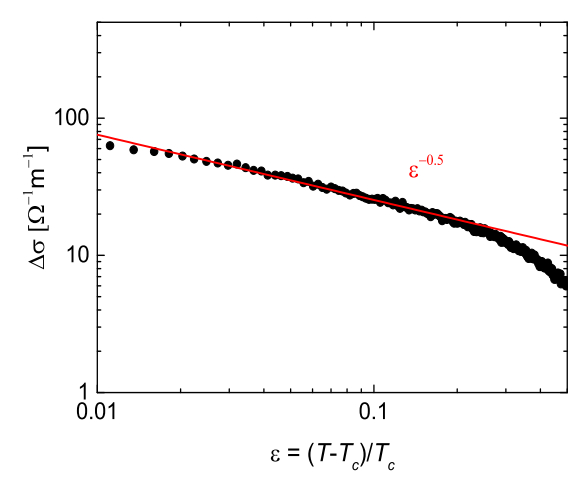

(d) $\mathrm{x}=0.25$

Figure 4.4: Fluctuation conductivity for all the superconducting samples with fitting to 3D regions.

\subsubsection{Field dependent resistivity in the superconduct- ing state}

The resistance was measured for different applied magnetic fields and shown in figure 4.5. It is apparent that the superconducting transition becomes broader and develops a tail with higher field. This tail is due to thermally assisted motion of vortices [138, 140, 141]. 


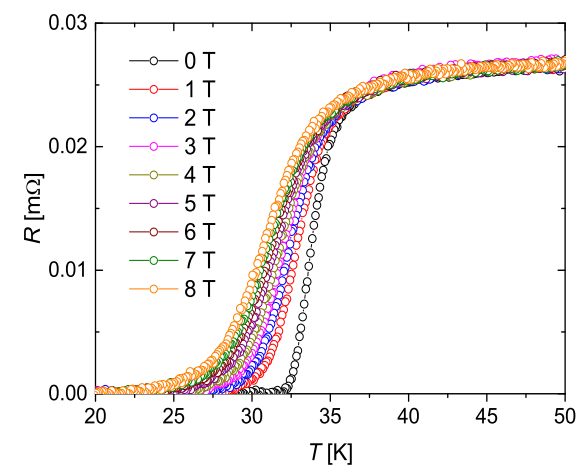

(a) $\mathrm{x}=0.13$

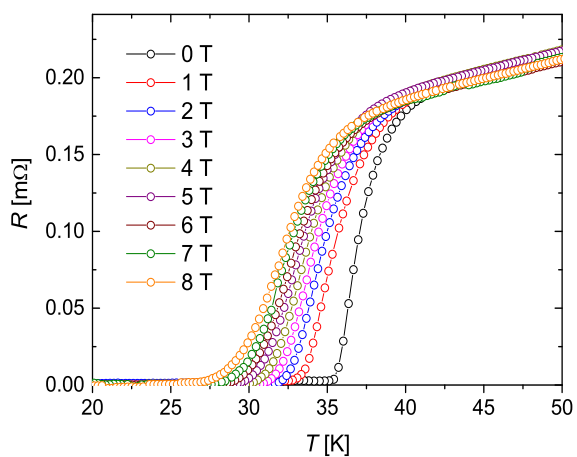

(c) $\mathrm{x}=0.20$

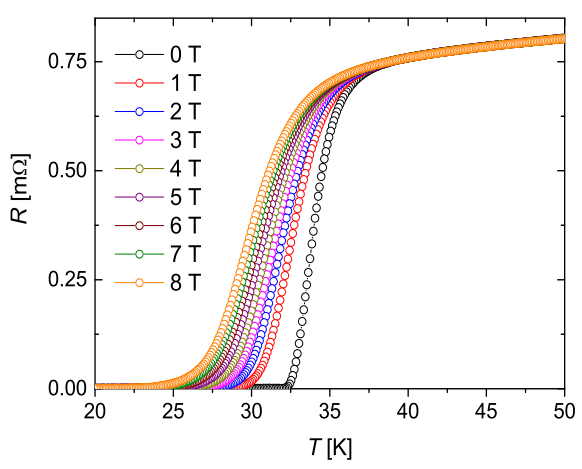

(b) $\mathrm{x}=0.15$

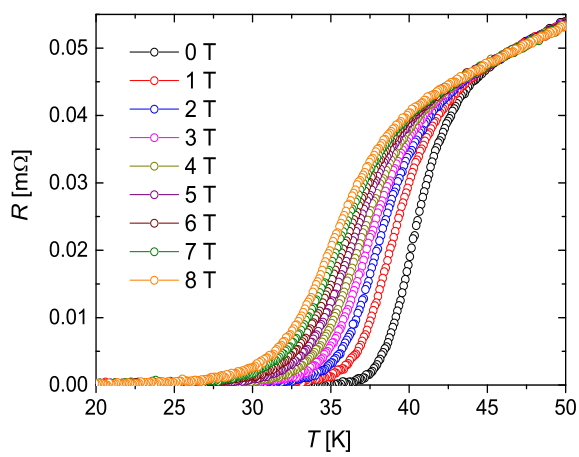

(d) $\mathrm{x}=0.25$

Figure 4.5: Temperature dependent resistance at around the superconducting transition at different applied fields.

It has been found in the iron-based superconductors that this vortex motion can be analyzed using the thermally assisted flux flow (TAFF) theory, where the resistivity can be written as [138, 140, 141]:

$$
\rho=\left(\frac{2 \nu_{0} L B}{J}\right) \exp \left(\frac{-J_{\mathrm{c} 0} B V L}{T}\right) \sinh \left(\frac{J B V L}{T}\right)
$$

with $\nu_{0}$ being the attempt frequency of a flux bundle hopping, $L$ is the hopping distance, $B$ is the magnetic induction, $J$ is the applied current density, $J_{\mathrm{c} 0}$ is the critical current density without flux creep, $V$ is the bundle volume and $T$ as the temperature. With the introduction of the thermal activation 
energy $U=J_{\mathrm{c} 0} B V L$ and $\rho_{\mathrm{C}}=\nu_{0} L B / J_{\mathrm{c} 0}$ equation 4.3 can be reduced, if $J$ is small enough and $J B V L / T \ll 1$, to

$$
\rho=\left(\frac{2 \rho_{\mathrm{C}} U}{T}\right) \exp \left(\frac{-U}{T}\right)=\rho_{0 f} \exp \left(\frac{-U}{T}\right)
$$

Because the prefactor $\left(\frac{2 \rho_{C} U}{T}\right)$ is only weakly temperature dependent, it is assumed to be a constant $\rho_{0 f}$ for the cuprate superconductors [141]. The activation energy can be written as $U(T, B)=U_{0}(B)(1-t)$ [140], where the reduced temperature, $t=T / T_{\mathrm{C}}$. The Arrhenius relation is then:

$$
\begin{gathered}
\ln (\rho(T, B))=\ln \left(\rho_{0}(B)\right)-\frac{U_{0}(B)}{T} \\
\ln \left(\rho_{0}(B)\right)=\ln \left(\rho_{0 f}\right)+\frac{U_{0}(B)}{T_{\mathrm{C}}}
\end{gathered}
$$

where the linear slope in the TAFF region of the plot of $\ln \rho$ vs $1 / T$ gives the activation energy $U_{0}(B)$ and the vertical intercept equals $\ln \rho_{0}(B)$ [140]. An example of the Arrhenius fit is shown in figure 4.6.

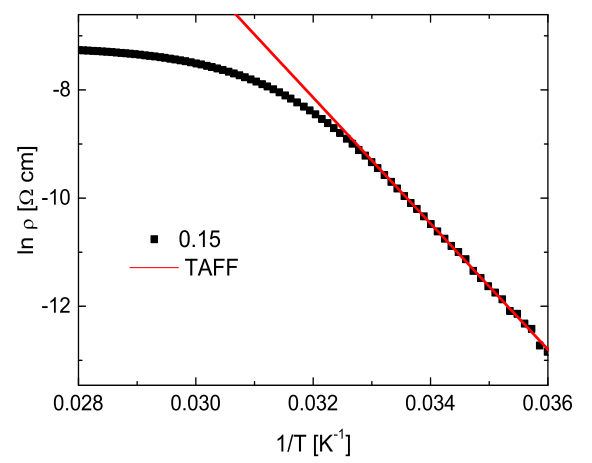

Figure 4.6: Example of Arrhenius plot for $\mathrm{x}=0.15$ at $2 \mathrm{~T}$ with a linear fit to the TAFF region.

Similar plots were done for all magnetic fields and for all the superconducting samples and the extracted linear fit values from the TAFF region are shown in figure 4.7. Such a plot allows the extraction of $T_{\mathrm{C}}$ and $\ln \rho_{0 f}$ from the linear fit using equation 4.6. The obtained values for the activation 
energy are shown in table 4.1 and plotted in figure 4.8, where it can be fitted to a power-law $U_{0}(B) \sim B^{n}$.

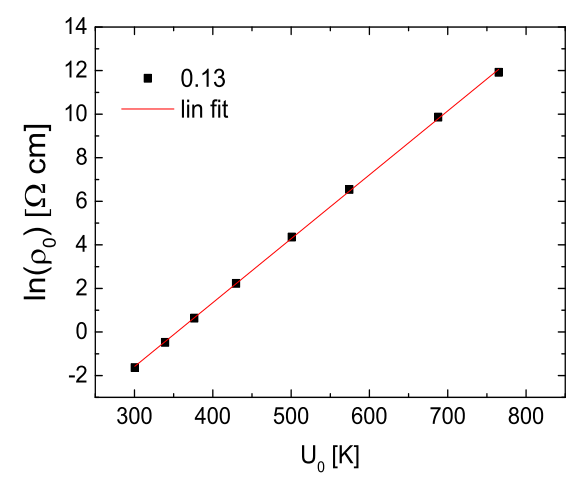

(a) $\mathrm{x}=0.13$

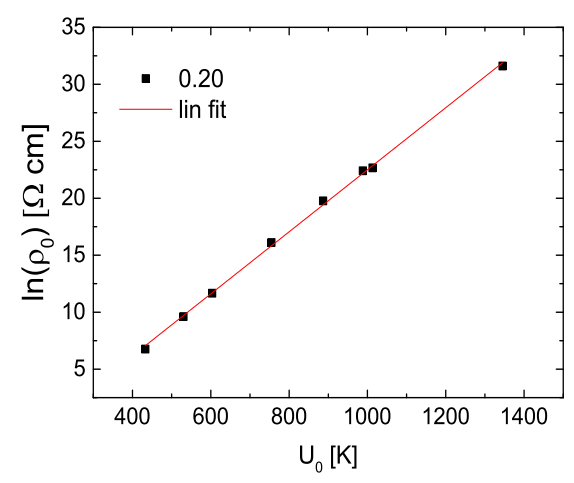

(c) $\mathrm{x}=0.20$

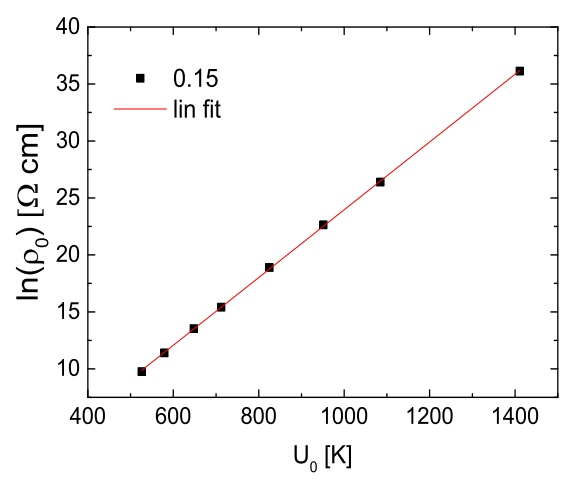

(b) $\mathrm{x}=0.15$

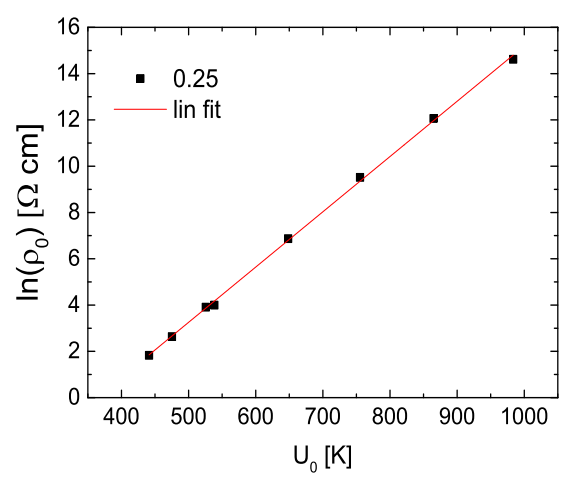

(d) $\mathrm{x}=0.25$

Figure 4.7: Arrhenius fits for Ce1111 for all superconducting samples. 


\begin{tabular}{|c|c|c|c|c|}
\hline $\mathrm{H}[\mathrm{T}]$ & 0.13 & 0.15 & 0.20 & 0.25 \\
\hline \hline 1 & $770(80)$ & $1410(140)$ & $1350(130)$ & $980(100)$ \\
\hline 2 & $690(70)$ & $1080(110)$ & $1010(100)$ & $860(90)$ \\
\hline 3 & $570(60)$ & $950(100)$ & $990(100)$ & $760(80)$ \\
\hline 4 & $500(50)$ & $830(80)$ & $890(90)$ & $650(60)$ \\
\hline 5 & $430(40)$ & $710(70)$ & $750(80)$ & $540(50)$ \\
\hline 6 & $380(40)$ & $650(60)$ & $600(60)$ & $530(50)$ \\
\hline 7 & $340(30)$ & $580(60)$ & $530(50)$ & $480(50)$ \\
\hline 8 & $300(30)$ & $530(50)$ & $430(40)$ & $440(40)$ \\
\hline
\end{tabular}

Table 4.1: Field dependence of $U_{0} / k_{\mathrm{B}}$ for all Ce1111 superconducting samples.

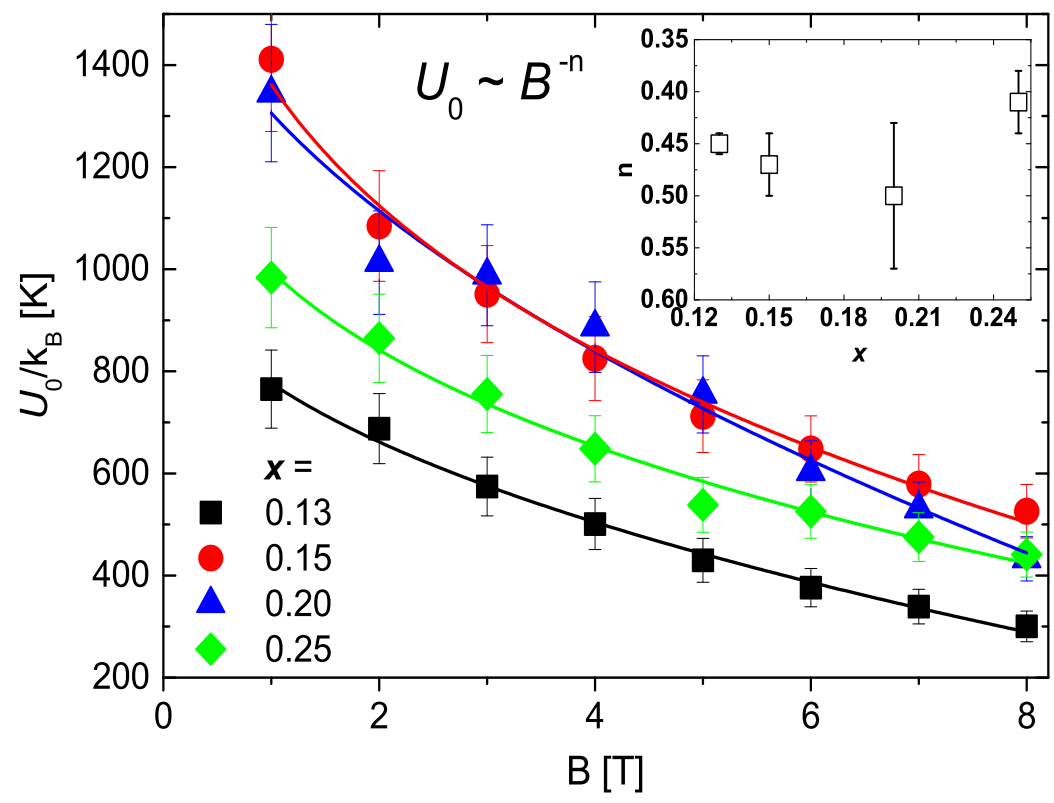

Figure 4.8: Field dependence of $U_{0} / k_{\mathrm{B}}$ for all Ce1111 superconducting samples and fits to a power-law. Inset: Fitted exponent $n$ of the power-law.

The field dependence of the activation energy $U_{0}(B)$ can be fitted to a power-law with an exponent which is close to $n \sim-0.5$ for all samples within the uncertainties. Previous studies of the activation energy, $U_{0}$, in iron-based superconductors [4, 130, 142] have followed a power-law dependence as well. 
An exponent value of $n \sim-0.5$ suggests a strong field dependency and collective-vortex pinning is dominant over that field region [143 145]. 


\subsection{Superconducting critical current density}

Another important property of superconductors, especially for potential practical applications, is the critical current density, $J_{\mathrm{C}}$. Magnetization fieldloops ( $M$ vs. $B$ ) were used to obtain the width of the irreversible magnetization $\Delta M=M^{-}-M^{+}$, with $\mathrm{M}^{+}$and $\mathrm{M}^{-}$being the branches of the field loop with increasing and decreasing field, respectively. An example of a field loop for $\mathrm{x}=0.15$ at $5 \mathrm{~K}$ is shown in figure 4.9 . The paramagnetic component of the magnetization, $M_{\mathrm{p}} \approx\left(M^{-}-M^{+}\right) / 2$, was subtracted from the field loop before using the Bean's critical state model [146, 147] to determine $J_{\mathrm{C}}$ :

$$
J_{\mathrm{C}}=\frac{3 \Delta M}{R}
$$

The average particle size, $R$, can be determined from scanning electron microscope (SEM) and XRD. All XRD patterns of $\mathrm{CeFeAsO}_{1-\mathrm{x}} \mathrm{F}_{\mathrm{x}}$ show small linewidth (figure A.1), which are to small for the Scherrer equation suggesting the overall particle size has to be larger than $1 \mu \mathrm{m}$. The SEM image (figure A.2 showed particle sizes in the range of $1 \mu \mathrm{m}$ to $100 \mu \mathrm{m}$. While the particle size has a big influence on the absolute value of $J_{\mathrm{C}}$, it does not change the studied dynamics. Hence, for all further calculations $R$ was assumed to be $\sim 10 \mu \mathrm{m}^{2}$.

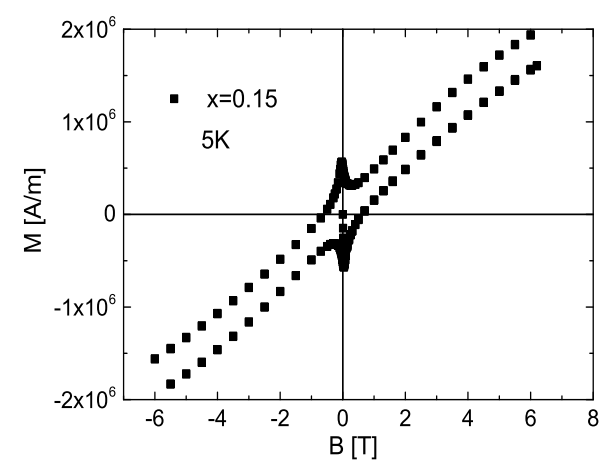

Figure 4.9: Magnetization field loop for $\mathrm{CeFeAsO}_{0.85} \mathrm{~F}_{0.15}$ at $5 \mathrm{~K}$.

\footnotetext{
${ }^{2}$ obtained values for $J_{\mathrm{C}}$ are in the same range as values obtained by other group for similar compounds
} 
$J_{C}$ is calculated for all four samples for $5 \mathrm{~K}$ and $10 \mathrm{~K}$ as shown in figure 4.10. The field dependence of $J_{C}$ for all four samples show three different field regions. A very low field region below $0.04 \mathrm{~T}$, an intermediate region from $0.04 \mathrm{~T}$ to a certain field, $B^{*}$, and a high field region above $B^{*}$.

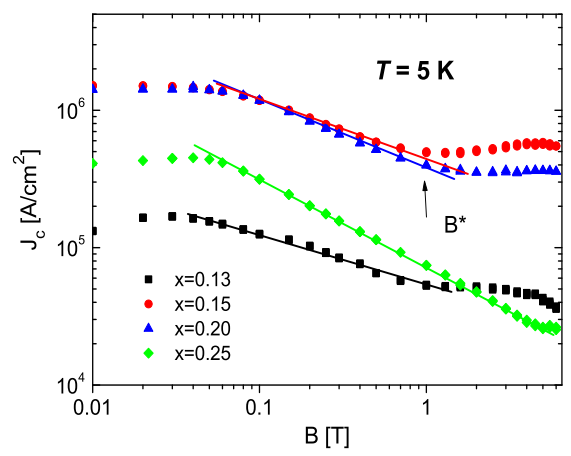

(a) $5 \mathrm{~K}$

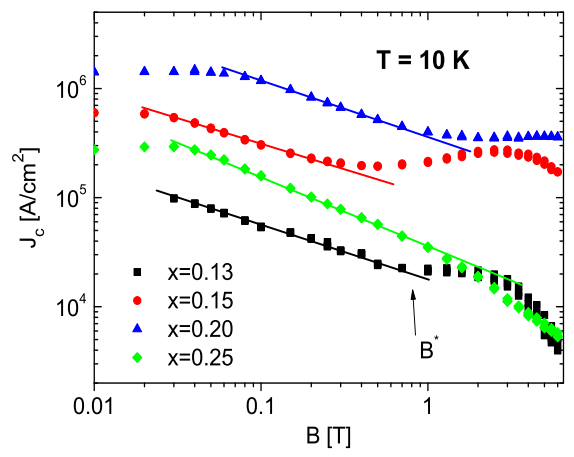

(b) $10 \mathrm{~K}$

Figure 4.10: Field dependence of the critical current density $J_{\mathrm{C}}$ for all four superconducting samples.

$J_{\mathrm{C}}$ is roughly field-independent in the low field region. The screening currents produce a self-field which is much higher than the external field $\left(B_{\text {self }} \gg B_{\text {ext }}\right)$. With increasing field above $0.04 \mathrm{~T} J_{\mathrm{C}}$ starts to decrease in the intermediate region, which is a common feature in both low temperature superconductors (LTSC) and high temperature superconductors (HTSC) [148]. In this region, $J_{\mathrm{C}}$ is linear in a log vs log plot as shown in figure 4.10. Hence, it can be fitted to an inverse power-law dependence on the field: $J_{\mathrm{C}}(B) \propto B^{-n}$ [149, which is shown as solid lines in figure 4.10. The exponent, $n$, of the inverse power-law increases with increasing doping from 0.39 for $\mathrm{x}=0.13$ to 0.62 for $\mathrm{x}=0.25$ shown in figure 4.11 , and display a correlation with $B^{*}$. 


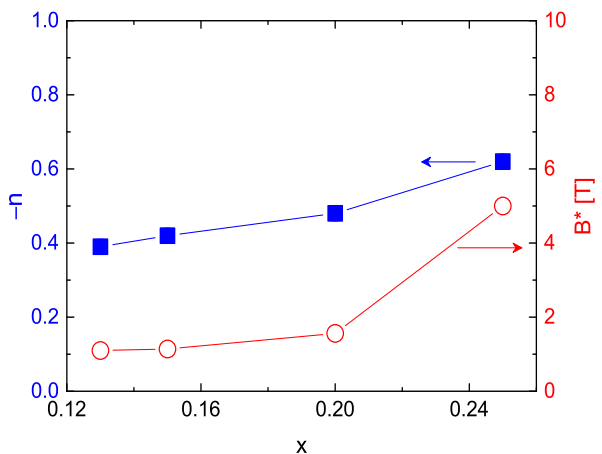

Figure 4.11: Doping dependence of $B^{*}$ together with the exponent, $n$, from the power-law fit.

These values are within the range observed in the cuprates $(n=0.25-1)$ [149, 150]. It is also believed that the existence of networks from weak links between grains and the thickness of the grains boundaries are responsible for the observed power-law behavior in HTSC [148, 149]. It is also noticeable above a certain critical field, $B^{*}$, that the power-law dependence of $J_{\mathrm{C}}$ vanishes. This is due to the suppression of weak links, which are responsible for the power-law dependency. Above $B^{*}$ they are driven to normal state and hence no longer contribute to $J_{\mathrm{C}}$ as observed in HTSC [149]. Furthermore, there is clear evidence of a peak-effect which is most obvious in the $\mathrm{x}=0.15$ sample. The peak-effect in $J_{\mathrm{C}}$ is observed in some HTSC [151, 152] as well as in the iron-based superconductors [130, 131, 153 156]. Possible explanations of the peak-effect include the magnetic field dependence of the vortex pinning potential and different crossover regimes in the vortex structure [154, 156]. 


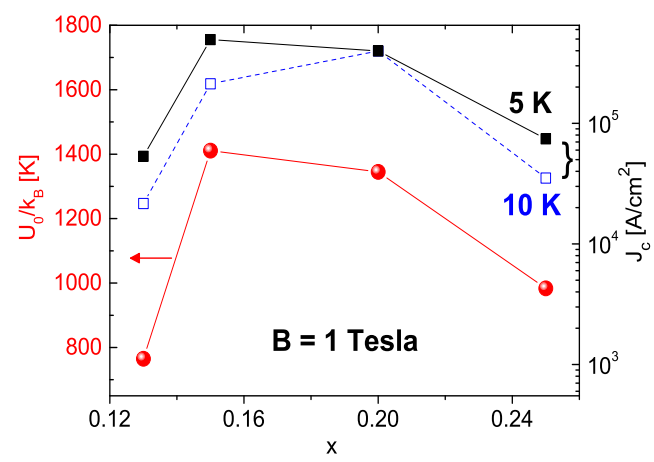

Figure 4.12: Doping dependence of $J_{\mathrm{C}}$ and the activation energy $U_{0}$.

The doping behavior of $J_{\mathrm{C}}$ is shown in figure 4.12 for an external magnetic field of $1 \mathrm{~T}$. It is apparent from the plot that $J_{\mathrm{C}}$ peaks somewhere between $\mathrm{x}=0.15$ and $\mathrm{x}=0.20$ for 5 and $10 \mathrm{~K}$. This behavior is contrary to a monotonic increase in $T_{\mathrm{C}}$ with increasing doping. A maximum in $J_{\mathrm{C}}$ in the slightly overdoped region was previously reported in the HTSC material $\mathrm{Y}_{1-\mathrm{x}} \mathrm{Ca}_{\mathrm{x}} \mathrm{Ba}_{2} \mathrm{Cu}_{3} \mathrm{O}_{7-\delta}$ [157]. It is attributed to an effect of the normal-state pseudogap which reduces the superconducting order parameter. $J_{\mathrm{C}}$ reaches a maximum at 0.19 holes per copper then decreases when the normal-state pseudogap vanishes. It is believed to reduce afterwards due to a reduction in the superconducting gap energy. There are reports of a normal state pseudogap in $\mathrm{LaFeAsO}_{1-\mathrm{x}} \mathrm{F}_{\mathrm{x}}$ [158 160] and $\mathrm{SmFeAsO}_{1-\mathrm{x}} \mathrm{F}_{\mathrm{x}}$ [161]. However, no observation of a normal-state pseudogap is reported for $\mathrm{CeFeAsO}_{1-\mathrm{x}} \mathrm{F}_{\mathrm{x}}$. It was also suggested that the normal-state pseudogap causes the decrease of $B_{\mathrm{c} 2}$ in $\mathrm{Y}_{1-\mathrm{x}} \mathrm{Ca}_{\mathrm{x}} \mathrm{Ba}_{2} \mathrm{Cu}_{3} \mathrm{O}_{7-\delta}$, which peaks around $p \sim 0.19$ hole doping where the normal-state pseudogap vanishes [162]. The opposite behavior is observed for $\mathrm{CeFeAsO}_{1-\mathrm{x}} \mathrm{F}_{\mathrm{x}}$, where no peak is noticed in $B_{\mathrm{c} 2}$ from $\mathrm{x}=0.13$ to $\mathrm{x}=0.25 \mathrm{~F}$-doping presented in the next section. Therefore, a pseudogap cannot explain the observed peak in $J_{\mathrm{C}}$ in our sample.

Figure 4.12 shows the doping dependence of $J_{\mathrm{C}}$ as well as the doping dependence of the activation energy. A peak in $U_{0}$ is seen at the same doping region as the peak in $J_{\mathrm{C}}$. This means that the maximum in $J_{\mathrm{C}}$ is due to the doping dependence of the depinning energy barrier. In a two-fluid 
flux creep model in granular samples a correlation occurs between $J_{\mathrm{C}}$ and $U_{0}$ where the collective response of vortices operates [143, 163]. In this model the relationship between $U_{0}$ and $J_{\mathrm{C}}$ is described by [143, 163]:

$$
U_{0}(t, B) \approx J_{\mathrm{C}}(0)\left[\frac{K g(t) t}{B}\right]
$$

with $t=T / T_{\mathrm{C}}$ the reduced temperature, $g(t)=4(1-t)^{3 / 2}$, and the constant $K=3 \sqrt{3} \Phi_{0} \beta /(2 c)$, where $c$ is the speed of light, $\Phi_{0}$ is the flux quantum and $\beta$ a numerical constant close to unity value. By plotting $J_{\mathrm{C}}$ (from figure 4.10 at $5 \mathrm{~K}$ and $1 \mathrm{~T}$ ) versus $U_{0}$ (from figure 4.8 at $1 \mathrm{~T}$ ) in figure 4.13 an excellent agreement between both values within the experimental uncertainties is observed, which is predicted by this two-fluid flux model.

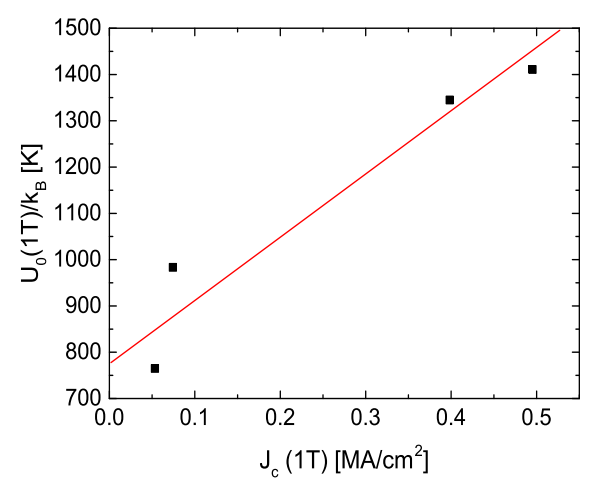

Figure 4.13: A linear relationship between $U_{0}$ and $J_{\mathrm{C}}$ within the two fluid flux creep model. 


\subsection{Upper critical field, $B_{\mathrm{c} 2}$}

The superconducting transition temperature, $T_{\mathrm{C}}$, is seen to reduce systematically with increasing magnetic field in all samples. The change in $T_{\mathrm{C}}$ from zero field to the maximum applied field $\left(\Delta T_{\mathrm{C}}=T_{\mathrm{C}}^{0 T}-T_{\mathrm{C}}^{8 T}\right)$ in figure 4.5 , for all the superconducting samples are compared. The observed change is small for $\mathrm{x}=0.13$ with $\Delta T_{\mathrm{C}}=1 \mathrm{~K}$ and increases with increasing $\mathrm{F}$-doping towards $\Delta T_{\mathrm{C}}=3.9 \mathrm{~K}$ for $\mathrm{x}=0.25$. This indicates a decrease in $B_{\mathrm{c} 2}$ with increasing doping. The method used in the literature [128, 130, 164, 165] to estimate the powder-averaged upper critical field is to use the temperature where the resistivity is reduced to $90 \%$ of the normal state resistivity. The obtained upper critical field versus temperature are shown in figure 4.14 .

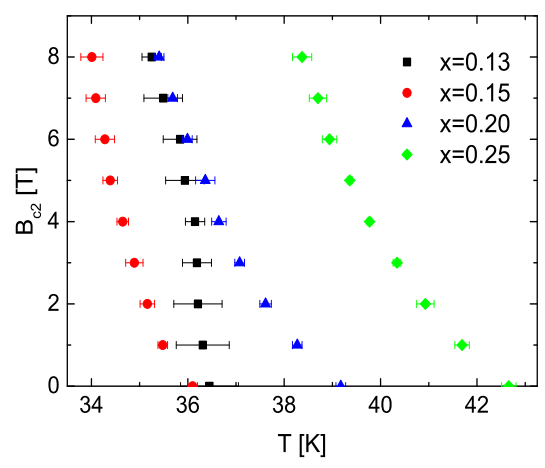

(a) Normal

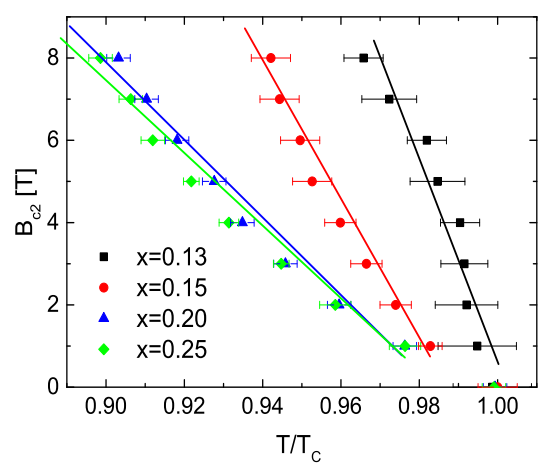

(b) Temperature scaled by $T_{\mathrm{C}}$.

Figure 4.14: Temperature dependence of the upper critical field, $B_{\mathrm{c} 2}$.

$B_{\mathrm{c} 2}$ is not linear over the whole field region and it deviates from the high field linearity moving closer to $T_{\mathrm{C}}[130]$. Therefore the average gradient above $1 \mathrm{~T}$ was used to estimate $d B_{\mathrm{c} 2} / d T$ from figure 4.14 . This method of obtaining $B_{\mathrm{c} 2}$ was previously demonstrated in the "linear analysis" to determine $B_{\mathrm{c} 2}$ for $\mathrm{YBa}_{2} \mathrm{Cu}_{3} \mathrm{O}_{7-\delta}$ [152] and $\mathrm{MgB}_{2}$ [166]. Figure 4.15 shows the gradient obtained with the described method, which decreases from $-6.1 \mathrm{~T} \mathrm{~K}^{-1}$ for $\mathrm{x}=0.13$ with increasing doping to $-2.1 \mathrm{~T} \mathrm{~K}^{-1}$ for $\mathrm{x}=0.25$. These values agree with values reported earlier by Shabazi et al. [130], but are slightly higher than those reported by Prakash et al. [129]. 


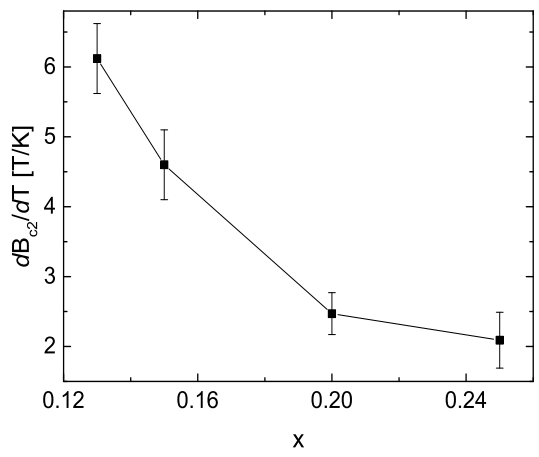

Figure 4.15: Doping dependence of $d B_{\mathrm{c} 2} / d T$.

From figure 4.15 it is possible to estimate the orbital limiting field at $0 \mathrm{~K}$, $B_{\mathrm{c} 2}^{\text {orb }}(0)$, through the single-band Bardeen-Cooper-Schrieffer (BCS) WerthamerHelfand-Hohenberg (WHH) formula [167]:

$$
B_{\mathrm{c} 2}^{\mathrm{orb}}(0)=0.693 T_{\mathrm{C}}\left|\frac{d B_{\mathrm{c} 2}}{d T}\right|_{T_{\mathrm{C}}}
$$

The obtained orbital limiting fields for $\mathrm{x}=0.13$ to $0.25 \mathrm{~F}$-doping are shown in figure $4.16 \mathrm{a}$. There is a noticeable decrease in $B_{\mathrm{c} 2}(0)$ with increasing doping until it becomes roughly independent of doping for $x \geq 0.20$.

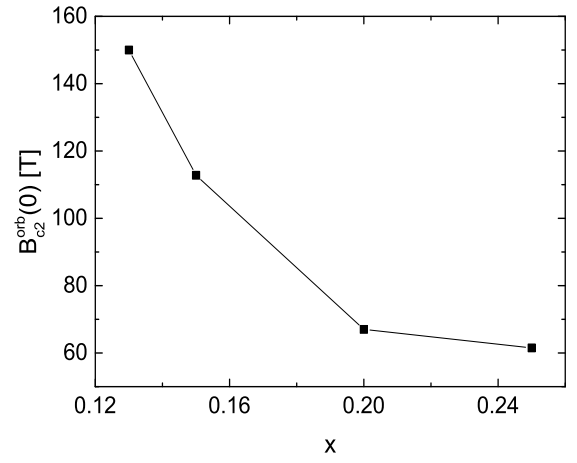

(a) Orbital limiting field $B_{\mathrm{c} 2}^{\mathrm{orb}}$

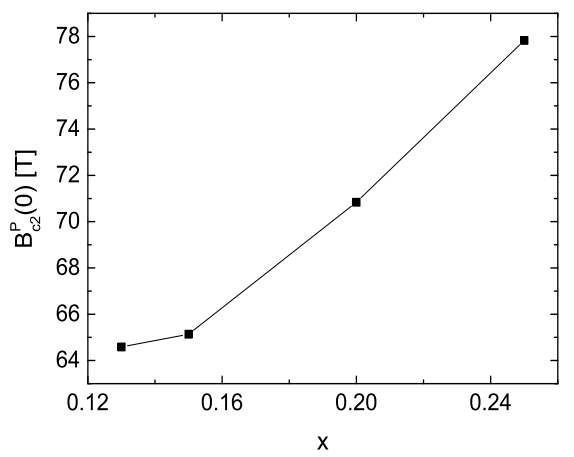

(b) Pauli limiting field, $B_{\mathrm{c} 2}^{\mathrm{P}}$

Figure 4.16: Limiting fields for $B_{\mathrm{c} 2}$ against doping.

In the BCS theory there exists a limit for $B_{\mathrm{c} 2}$ which a BCS superconductor 
cannot exceed. This limit is call the Pauli limiting field, $B_{\mathrm{c} 2}^{\mathrm{P}}(0)$, and it can be defined as the field, where the superconducting condensate energy equals the normal state Zeeman energy. The BCS $B_{\mathrm{c} 2}^{\mathrm{P}}(0)$ can be calculated from $B_{\mathrm{c} 2}^{\mathrm{P}}(0)=1.84 \mu_{0} T_{C}$ and these values are shown in figure $4.16 \mathrm{~b}$. It is shown in previous studies of iron-based superconductors that several of them can exceed the Pauli limiting field [128, 168, 169]. The relative importance of spin-orbit versus Pauli paramagnetic pair-breaking can be qualitatively gauged by the Maki parameter, $\alpha$ [144,

$$
\alpha=\sqrt{2} \frac{B_{\mathrm{c} 2}^{\mathrm{orb}}(0)}{B_{\mathrm{c} 2}^{\mathrm{P}}(0)}
$$

At low orbital limiting field, $\alpha$ can reach values close to 1 , which is observed for $\mathrm{x}=0.20$ and above. The value of $\alpha$ can even exceed 1 as shown in figure 4.17 for a doping of $\mathrm{x}=0.15$ and below. Such large $\alpha$ values suggest that pairbreaking is not limited to Pauli limiting effects but orbital effects have to be included. Large $\alpha$ values have been observed in other RFeAsO superconductors with $\mathrm{R}=\mathrm{La}$ [168] and $\mathrm{Sm}$ [169]. They suggest that spin-orbit scattering should be included to calculate $B_{\mathrm{c} 2}$. However, it looks like the single-band model is insufficient to get a satisfactory result. A two-gap model seems to be required to describe $B_{\mathrm{c} 2}$ in the iron-based superconductors [168, 169] although that can depend on the orientation of the ab-plane with respect to the applied magnetic field [169].

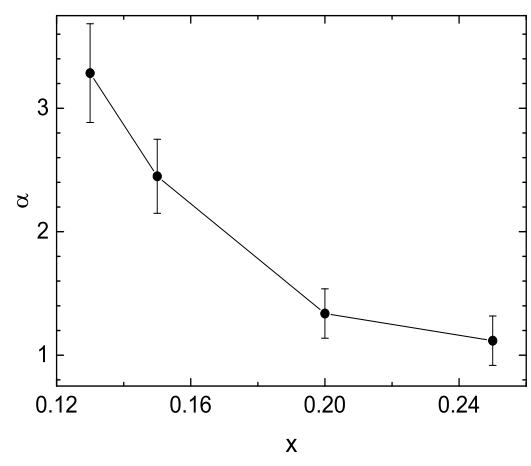

Figure 4.17: Maki parameter, $\alpha$, against doping. 


\subsection{Irreversible field, $B_{\text {irr }}$}

The irreversible magnetic field, $B_{\text {irr }}$, is commonly used to determine an upper limit for $J_{\mathrm{C}}$. $B_{\text {irr }}$ for all the superconducting samples of $\mathrm{CeFeAsO}_{1-\mathrm{x}} \mathrm{F}_{\mathrm{x}}$ was determined from the difference between the field-cooled (FC) and zero-fieldcooled (ZFC) magnetization, $\Delta \mathrm{M}$ (figure 4.18a), at fixed applied magnetic fields up to $6 \mathrm{~T}$. $B_{\text {irr }}$ is defined as the magnetic field at which $\Delta \mathrm{M}$ fell to less than $140 \mathrm{~A} \mathrm{~m}^{-13}[170]$.

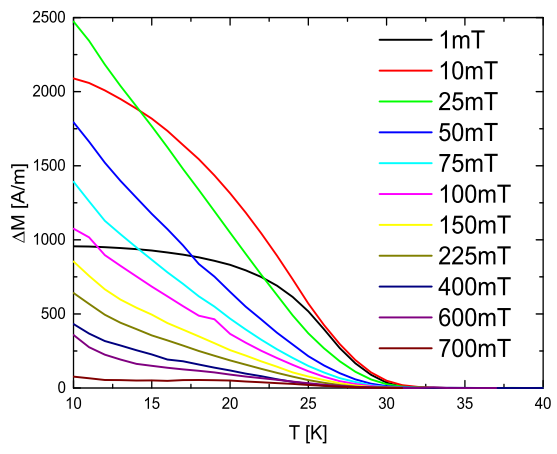

(a) Difference between FC and ZFC, $\Delta \mathrm{M}$, for $\mathrm{x}=0.13$ and different applied fields.

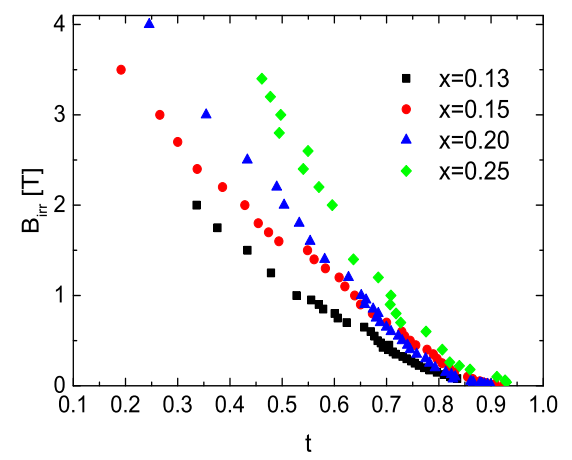

(b) Irreversible field, $B_{\text {irr }}$, against the reduced temperature, $t$.

Figure 4.18: Irreversible magnetic field, $B_{\mathrm{irr}}$.

Figure $4.18 \mathrm{~b}$ shows the obtained $B_{\text {irr }}$ for all four samples plotted against the reduced temperature, $t=T / T_{\mathrm{C}}$. In the HTSC cuprates there is a critical behavior commonly observed around $T_{\mathrm{C}}$. Therefore, the data from figure $4.18 \mathrm{~b}$ is replotted on a log-log scale in figure 4.19, which provides more details about the vortex dynamics especially their melting behavior. The data can be described with a power-law:

$$
B_{\text {irr }} \propto(1-t)^{n}
$$

where the exponent $n$ is an indication of the different vortex dynamics. An exponent of $n \sim 1.5$ was found for $\mathrm{MgB}_{2}$ [171] which is due to a collective

\footnotetext{
${ }^{3} 1 \mathrm{mT}=7.958 \times 10^{-6} \mathrm{~A} \mathrm{~m}^{-1}$
} 
pinning commonly observed in LTSC. An exponent of $n=1.5-2$ was found in HTSC $\mathrm{Bi}_{2-\mathrm{x}} \mathrm{Pb}_{\mathrm{x}} \mathrm{Sr}_{2} \mathrm{Ca}_{2} \mathrm{Cu}_{3} \mathrm{O}_{10+\mathrm{y}}$ [170] and $\mathrm{Y}_{1-\mathrm{x}} \mathrm{Pr}_{\mathrm{x}} \mathrm{Ba}_{2} \mathrm{Cu}_{3} \mathrm{O}_{6+\mathrm{y}}$ [152, 172 ] for $t>0.8$, which is associated with a $3 \mathrm{D}$ vortex lattice-melting model. In $\mathrm{CeFeAsO}_{1-\mathrm{x}} \mathrm{F}_{\mathrm{x}}$ there are two regions with different exponent values. The regions are separated at around $t=0.8$, with values above 0.8 being $n \geq$ 3 and values below 0.8 following a power-law dependence around $n \sim 2$. This is associated with $3 \mathrm{D}$ vortex lattice-melting model as it is observed in other low anisotropy iron-based superconductors [147, 173, 174] and in the aforementioned HTSC cuprates [152, 170, 172].

\begin{tabular}{|c|c|c|c|}
\hline doping & $T_{\mathrm{C}}[\mathrm{K}]$ & $n$ for $t<0.8$ & $n$ for $t>0.8$ \\
\hline \hline 0.13 & 33.5 & 1.74 & 3.08 \\
\hline 0.15 & 35.0 & 2.02 & 3.84 \\
\hline 0.20 & 38.1 & 2.24 & 6.78 \\
\hline 0.25 & 42.5 & 2.68 & 4.70 \\
\hline
\end{tabular}

Table 4.2: Fitted exponent values of the power-law dependence of the irreversible field $B_{\text {irr }}$.

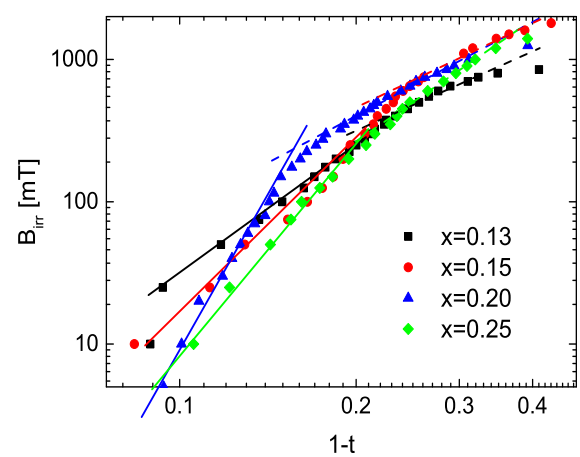

Figure 4.19: Plot of $\log \left(B_{\text {irr }}\right)$ vs $\log (1-t)$ for $\mathrm{CeFeAsO}_{1-\mathrm{x}} \mathrm{F}_{\mathrm{x}}$. Also plotted are fits to $(1-t)^{n}$ with $n \sim 1.7$ to 2.7 for $t<0.8$ (dashed lines) and $n>3$ for $t>0.8$ (solid lines).

To investigate the doping dependent behavior of $B_{\text {irr }}$, one can scale the irreversible field against $B_{\mathrm{c} 2}(0)$ as shown in figure 4.20 by the zero temperature 
$B_{\mathrm{c} 2}$ :

$$
\frac{B_{\mathrm{irr}}(T, p)}{B_{\mathrm{c} 2}(0, p)}=\beta \cdot(1-t)^{n}
$$

The scaled $B_{\text {irr }}$ increases with increasing doping (figure 4.20) and the obtained power-law exponent values are the same as shown in figure 4.19.

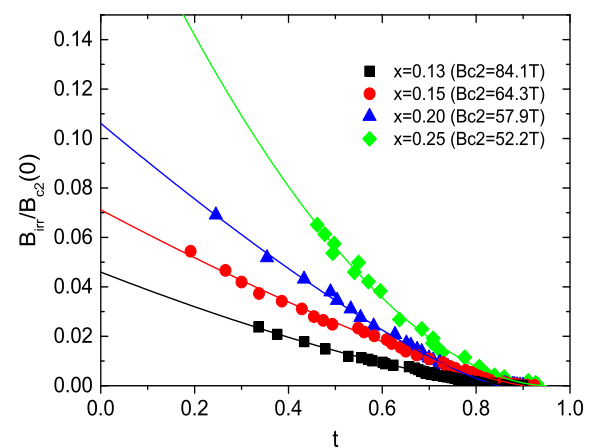

Figure 4.20: Scaling of $B_{\text {irr }}$ by $B_{\mathrm{c} 2}(0)$ and the fit to a power-law dependent behavior. 


\subsection{Susceptibility}

Susceptibility measurements were performed to determine the effective moment of non-superconducting $\mathrm{CeFeAsO}_{0.93} \mathrm{~F}_{0.07}$. This particular sample was chosen because it is close to the crossover from SDW transition and superconductivity as previously shown in section 4.3.1. Therefore it is neither superconducting and should not be magnetically ordered, although it will be shown later that there is a SDW transition around $20 \mathrm{~K}$ (chapter 5). The effective moment (in $\mu_{\mathrm{B}}$ ) of Ce in the paramagnetic region can be calculated from [18]:

$$
P_{\mathrm{eff}}^{\mathrm{Ce}}=g(J L S) \sqrt{J(J+1)}
$$

where $g$ is the Landé g-factor and $L, S$ and $J$ are the orbital, spin and total angular momentum. Ce has a standard electron configuration of $4 f^{1}$, which gives $s=1 / 2, L=3$ and $J=5 / 2$. With that $g(J L S)$ can be calculated from [18],

$$
g(J L S)=\frac{3}{2}+\frac{1}{2} \frac{S(S+1)-L(L+1)}{J(J+1)}
$$

giving $g(J L S)$ for $\mathrm{Ce}=6 / 7$. Therefore, the theoretical free effective moment for Ce is $P_{\mathrm{eff}}^{\mathrm{Ce}}=2.54 \mu_{\mathrm{B}}$.

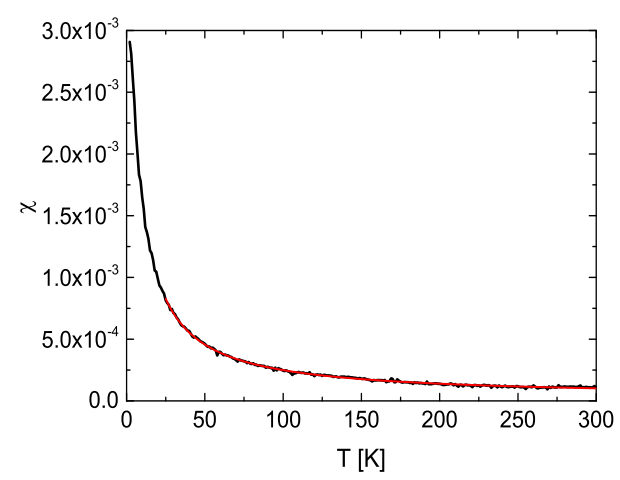

Figure 4.21: Temperature dependent susceptibility of $\mathrm{CeFeAsO}_{0.93} \mathrm{~F}_{0.07}$.

The susceptibility was measured in a field of $B=6 \mathrm{~T}$ on a sample with a mass of $40.1 \mathrm{mg}$. The results are shown in figure 4.21 together with a fit to a Curie-Weiss (CW) temperature dependent behavior above $25 \mathrm{~K}$, which is 
above the iron and the cerium magnetic ordering temperatures. The obtained fitted parameters gave a $\mathrm{CW}$ temperature of $-4 \mathrm{~K}$ in good agreement with the ordering of the Ce moments, a value of $\chi_{0}=2.5 \times 10^{-5}$ for the temperature independent part of the susceptibility and a CW constant of 0.0234. With the fitted $\chi_{0}$ value the temperature dependent of $1 /\left(\chi-\chi_{0}\right)$ can be plotted, which shows a linear dependency over the whole measured temperature region as shown in figure 4.22 .

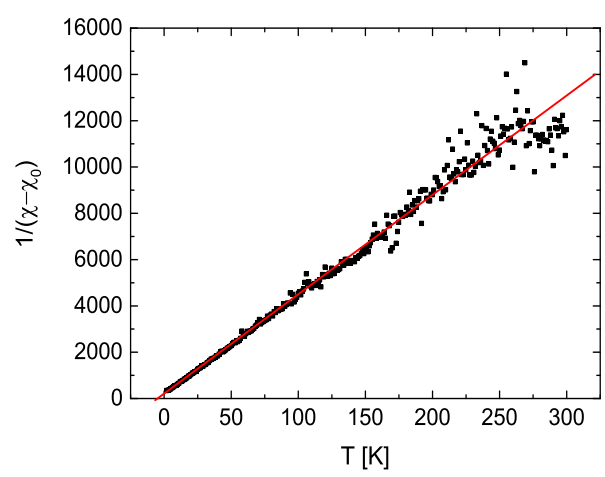

Figure 4.22: Linear temperature dependent behavior of $1 /\left(\chi-\chi_{0}\right)$ for $\mathrm{CeFeAsO}_{0.93} \mathrm{~F}_{0.07}$.

The effective magnetic moment of $\mathrm{CeFeAsO}_{0.93} \mathrm{~F}_{0.07}$ can then be calculated with equation 3.5. $N$ is 2 (two formula per unit cell) [175] and the volume $V$ can be calculated from the known density $\rho=6.981 \mathrm{~g} / \mathrm{cm}^{3}$ through equation 3.6 with a empirical molar mass of $287.092 \mathrm{~g} \mathrm{~mol}^{-1}$. Equation 3.5 gives an effective moment per cerium of $P_{\mathrm{eff}}^{\mathrm{Ce}}=1.57(1) \mu_{\mathrm{B}}$ for the least-squares fit from figure 4.21. This is smaller than the predicted value for the free magnetic moment of $\mathrm{Ce}^{3+}$. The measured values could be lower, because this calculation ignored the contribution from iron moments. The iron effective moment is not known although there is an ordered moment of $P^{\mathrm{Fe}}=\sim 0.4-0.8 \mu_{\mathrm{B}}$ [145, 159, 176 178] in the SDW state. A possible explanation is that there are Zener-type [179] antiferromagnetic interactions between the itinerant carriers in the FeAs plane and the localized Ce moments. These interactions are a special form of superexchange interactions called double exchange or Zenertype, where electrons can be exchanged between two metal ions of equal or 
+2 charge state via a connecting ligand. 


\subsection{Summary and conclusions}

The electronic transport and magnetic properties of $\mathrm{CeFeAsO}_{1-\mathrm{x}} \mathrm{F}_{\mathrm{x}}$ were reported in this chapter. It was found that the resistivity for the nonsuperconducting sample $(\mathrm{x}=0.07)$ follows a linear temperature dependence often called "strange metal". The superconducting samples show a Fermi liquid temperature dependence above the superconducting transition temperature and a three-dimensional fluctuation conductivity is observed near $T_{\mathrm{C}}$. A crossover to two-dimensional behavior happens further away from $T_{\mathrm{C}}$ with increasing fluoride content due to a growing coherence length. The superconducting region of the resistivity was analyzed within the thermally activated flux flow model and vortex motion could be observed within the model. The activation energy and the critical current density $J_{\mathrm{C}}$ are observed to peak around the optimal doping region. A correlation between these two values is observed, which is accounted for in the two-fluid flux creep model. The irreversibility field is higher for larger fluoride content and the data could be described by a power-law. An exponent of around 2 within the powerlaw was interpreted as 3D vortex lattice melting as it was observed in some cuprates and other low anisotropy pnictides. An orbital limiting field, $B_{\mathrm{c} 2}^{\text {orb }}$, of up to $150 \mathrm{~T}$ for $\mathrm{x}=0.13$ was calculated using the WHH model. The Maki parameter reaches the largest value for $\mathrm{x}=0.13$ and decreases with increasing $\mathrm{x}$ reaching a value still larger than 1 for $x \geq 0.20$. This indicates that an orbital limiting effect alone is not enough to describe the pair-breaking effect and the Pauli limiting effect has to be included. The effective moment per formula unit was found to be lower than expected for the free moment of Ce. This may be due to Zener-type interactions between the local Ce moments and the itinerant carriers. 


\section{Chapter 5}

\section{${ }^{75}$ As NMR on $\mathrm{CeFeAsO}_{1-\mathrm{x}} \mathrm{F}_{\mathrm{X}}$ : Spin and charge inhomogeneities}

\subsection{Introduction}

The magnetic ordering of $\mathrm{Fe}$ as well as Ce allows a unique opportunity to study their relationship to superconductivity. A lot of attention is focused on the lower doping region, due to a better sample purity as well as the transition between superconductivity and magnetism with a possible coexistence of both phases. But the higher doping region also has a lot to offer. A good tool to access such information is through NMR, where NMR peaks from more than one As environment can appear and there can also be two different spinlattice relaxations times, $T_{1}[7,176,180$ 183]. Performing such measurements can reveal critical insights into the magnetic and electronic properties of $\mathrm{CeFeAsO}_{1-\mathrm{x}} \mathrm{F}_{\mathrm{x}}$, and provide an additional source of information to understand this system. 


\subsection{NMR on CeFeAsO}

The undoped parent material is non-superconducting and undergoes a structural transition from tetrahedral to octahedral around $155 \mathrm{~K}$ [2]. The structural change results in a developing asymmetry $(\eta>0)$, but those changes are small enough to have no significant effect on the NMR spectra. However, at around $140 \mathrm{~K} \mathrm{CeFeAsO}$ undergoes a SDW transition which has large effect on the ${ }^{75}$ As NMR Spectra. The usual spectra of a $I=3 / 2$ nuclei (two satellite transition (ST) peaks and one central transition (CT) peak) double and shift by a factor proportional to the internal field of the SDW magnetic ordering. Changes in $T_{1}$ and $T_{2}$ are also expected.

\subsubsection{Temperature-dependence of ${ }^{75} \mathrm{As}$ NMR spectra}

The room temperature (RT) central transition (CT) could be found at 85.875(5) MHz in the $c \| B_{0}$ direction (figure 5.1a) and at a slightly higher frequency of $86.14(1) \mathrm{MHz}$ for $a b \| B_{0}$ (figure 5.1b), due to the 2nd order quadrupole contribution. The magnetic shift $K$ can be calculated using equations 2.16 and 2.17. This results in a shift of $K_{c}=0.306(1) \%$ and $K_{a b}=0.616(1) \%$.

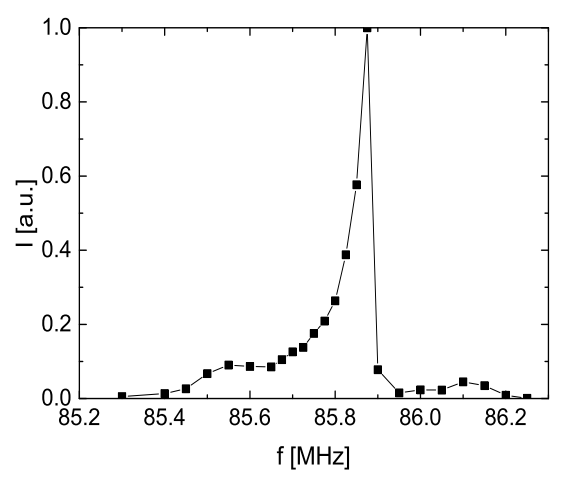

(a) $c \| B_{0}$

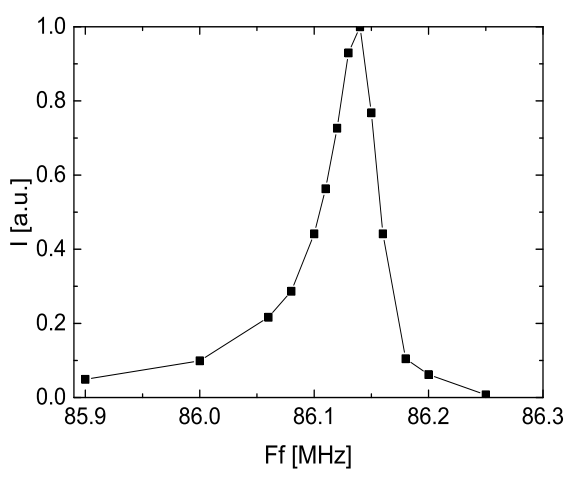

(b) $\mathrm{ab} \| \mathrm{B}_{0}$

Figure 5.1: ${ }^{75} \mathrm{As}$ NMR spectra of the central transition from CeFeAsO at room temperature. 
The NMR spectra at $295 \mathrm{~K}$ for $c \| B_{0}$ over a wider frequency range can be seen in figure 5.2. The lower and higher frequency peaks are the ${ }^{75} \mathrm{As}$ satellites. Their separation can be used to estimate the quadrupole frequency $f_{\mathrm{q}}=9.9 \mathrm{MHz}$.

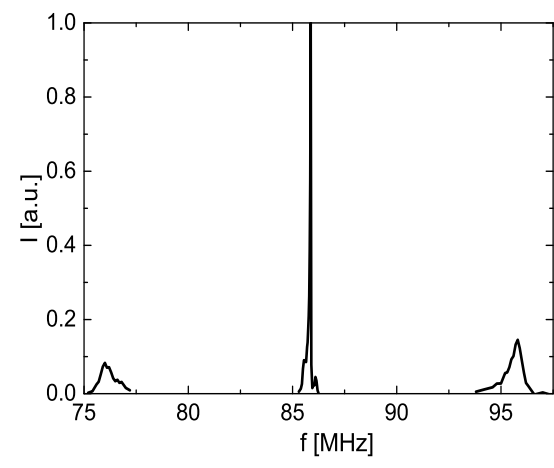

Figure 5.2: ${ }^{75} \mathrm{As}$ NMR spectra of Figure 5.3: CeFeAsO ${ }^{75} \mathrm{As}$ NMR $\mathrm{CeFeAsO}$ at room temperature for $c \| B_{0}$ including satellite transitions.

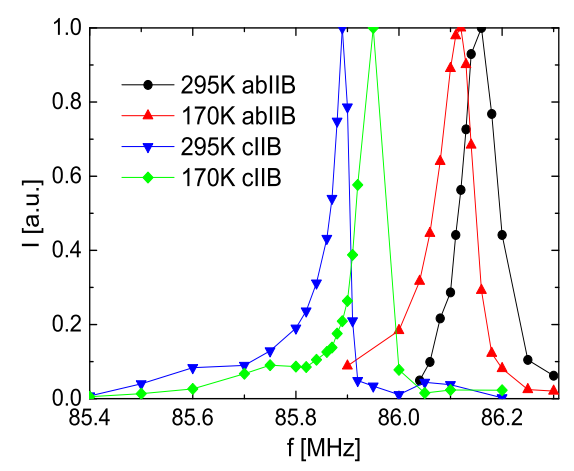
spectra for both orientations at $170 \mathrm{~K}$ and room temperature.

There is no significant change to the spectra with temperature above the SDW transition, as it is show by the spectra at $170 \mathrm{~K}$ in figure 5.3. Below the SDW transition the Fe moments order antiferromagnetically in a stripe-type fashion [2]. This antiferromagnetic ordering results in an additional internal field contribution, $B_{\text {int }}$, which shifts the whole spectrum. Furthermore, there are now two different As-environments, due to either a positive or negative net effect at the As position, which results from a non-zero hyperfine contribution only in the $c$-direction similar to that observed in $\mathrm{BaFe}_{2} \mathrm{As}_{2}[184$ ] and $\mathrm{SrFe}_{2} \mathrm{As}_{2}$ [185]. Therefore, the expectation is to observe a spectra containing two sets of three lines. Those lines could indeed be measured as shown in figure 5.4. An interesting feature to note is that the splitting increases with decreasing temperature, which is likely to be due to spin waves. 


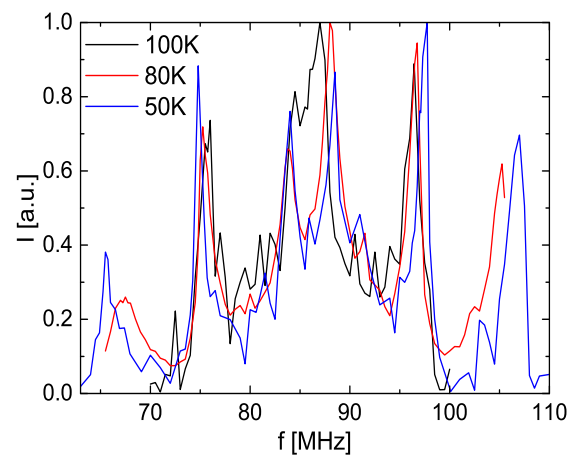

Figure 5.4: CeFeAsO ${ }^{75} \mathrm{As}$ NMR Spectra for $c \| B_{0}$ below the SDW transition.

A non-zero hyperfine contribution only in the $c$-direction also means, that there should be no splitting in the $a b$-direction. Indeed this is what was found. When cooling through the SDW transition the only change in the NMR spectra in the $a b$-direction is an increase in the line broadening as shown in figure 5.5 and a small NMR shift.

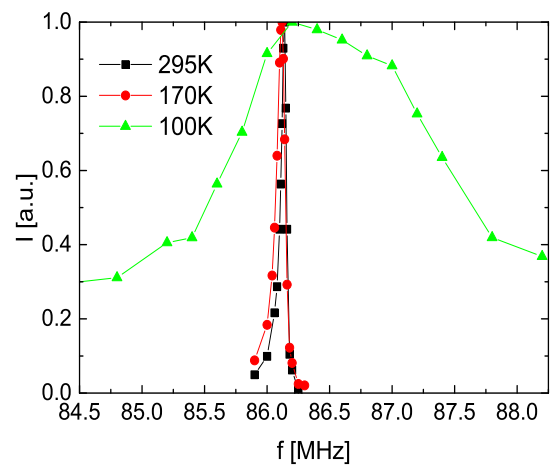

Figure 5.5: CeFeAsO ${ }^{75} \mathrm{As}$ NMR spectra for $a b \| B_{0}$ above and below the SDW transition.

The additional internal field, $B_{\text {int }}$, caused by the ordered Fe moments can be calculated. The behavior of the splitting indicates that the internal field is parallel to $c$, which means there is an effective field $B_{\text {eff }}=B_{0} \pm B_{\text {int }}$ in the $c$-direction. No effect is observed perpendicular to $c$ as shown in 
figure 5.5. The calculated internal fields $B_{\text {int }}$ are shown in figure 5.6 and table 5.1. These values of $B_{\text {int }}^{c}$ are similar to those observed for $\mathrm{BaFe}_{2} \mathrm{As}_{2}$ and lower then those for $\mathrm{SrFe}_{2} \mathrm{As}_{2}[185]$. However, errors are larger for low temperatures, because no full spectra was measured at low temperature and only a few frequency steps were used to check the maximum position for the relaxation measurements.

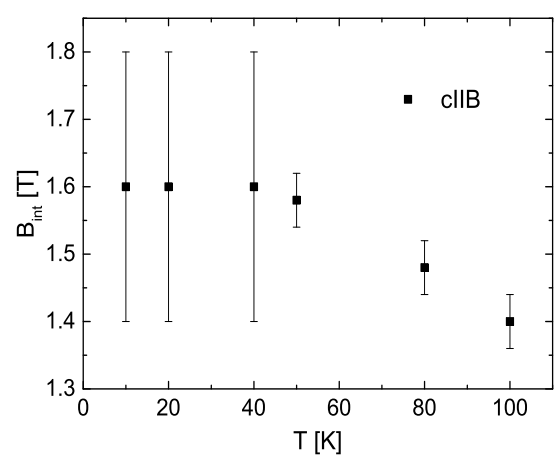

Figure 5.6: Internal field, $B_{\text {int }}$, below the SDW transition.

\begin{tabular}{|c|c|}
\hline Temperature $[\mathrm{K}]$ & $B_{\text {int }}^{c}[\mathrm{~T}]$ \\
\hline \hline 100 & $1.40(4)$ \\
\hline 80 & $1.48(4)$ \\
\hline 50 & $1.58(4)$ \\
\hline 40 & $1.6(2)$ \\
\hline 20 & $1.6(2)$ \\
\hline 10 & $1.6(2)$ \\
\hline
\end{tabular}

Table 5.1: Internal field, $B_{\text {int }}^{c}$, in the $c$-direction below the SDW transition.

\subsubsection{Temperature dependence of the spin-lattice re- laxation in $\mathrm{CeFeAsO}$}

The spin lattice relaxation times were all measured using a 2D-inversionrecovery-sequence with an additional Hahn-echo, as described in section 2.6.2. Typical values for $T_{1}$ are around $10 \mathrm{~ms}$ and below. Therefore, the 
typical values for $t$ (duration) are up to $50 \mathrm{~ms}$. Figure 5.7 shows the result of $T_{1}$ measured for the $\mathrm{CT}$ in $c \| B_{0}$ at room temperature (RT), which is roughly $295 \mathrm{~K}$. Similar measurements were done for several temperatures in both orientations and the results are presented in table 5.2 and figure $5.8 \mathrm{a}$.

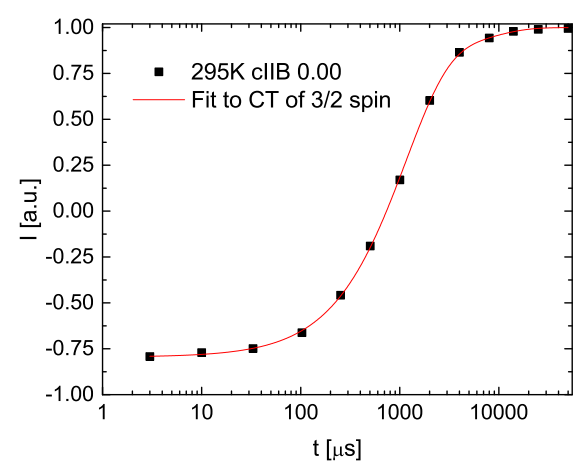

Figure 5.7: Integrated intensities of the echo for different durations of $t$ at $295 \mathrm{~K}$ in $c \| B_{0}$ where the fit to equation 2.19 is in red.

\begin{tabular}{|c|c|c|}
\hline Temperature $[\mathrm{K}]$ & $T_{1}^{c}[\mathrm{\mu s}]$ & $T_{1}^{a b}[\mathrm{\mu s}]$ \\
\hline \hline 295 & $6720(80)$ & $7170(90)$ \\
\hline 170 & $3610(30)$ & $6140(80)$ \\
\hline 100 & & $6200(500)$ \\
\hline 80 & $2060(130)$ & $8000(600)$ \\
\hline 50 & $920(80)$ & $7250(250)$ \\
\hline 40 & $1100(130)$ & $7800(250)$ \\
\hline 20 & $1000(60)$ & $10400(300)$ \\
\hline 10 & $1700(100)$ & $19600(2100)$ \\
\hline
\end{tabular}

Table 5.2: $T_{1}$ for CeFeAsO at different temperatures and both orientations and the experimental uncertainty.

\footnotetext{
${ }^{1}$ the inversion of the recovery sequence is not perfect because the $\tau_{\pi / 2}$ is already $13 \mu \mathrm{s}$ and thus the excitation width does not cover the whole CT.
} 


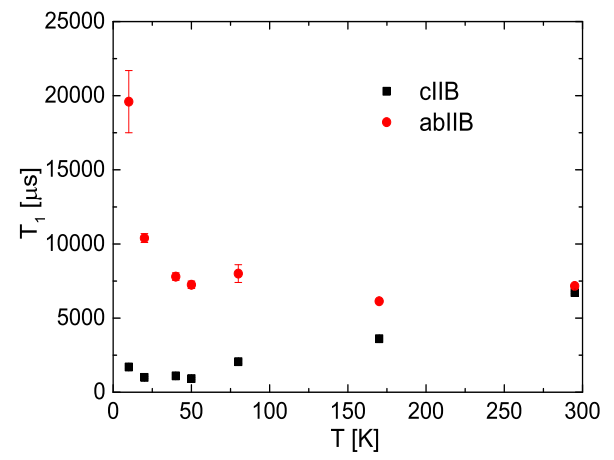

(a) $T_{1}$

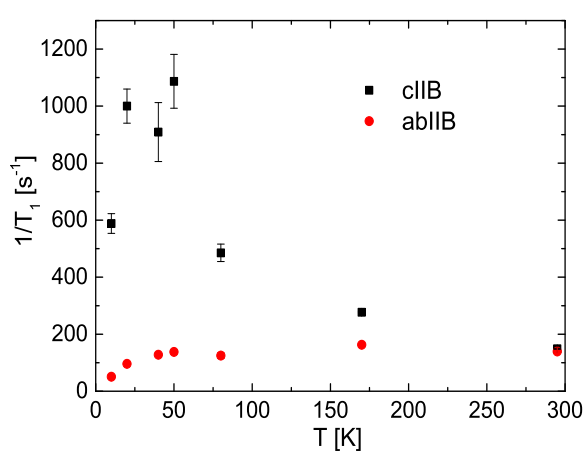

(b) $1 / T_{1}$

Figure 5.8: Temperature dependence of the spin-lattice relaxation time $T_{1}$ and rate, $1 / T_{1}$

There are no large changes in the spin-lattice relaxation rate near the SDW transition temperature, which might be at least expected for $1 / T_{1}^{c}$ because it couples to the ab-plane spin fluctuations. $1 / T_{1}^{c}$ is found to increase with increasing temperature, while $1 / T_{1}^{a b}$ decreases. For both orientations the spin-lattice relaxation rate is lower at the lowest temperatures and this might be due to an ordering of Ce moments around $5 \mathrm{~K}$ [2]. The resultant anisotropy ratio, $R_{1}$ :

$$
R_{1}=\frac{T_{1, a b}^{-1}}{T_{1, c}^{-1}}
$$

is found to decrease with decreasing temperature as shown in figure 5.9 and reaches a value below 0.1 at $10 \mathrm{~K}$. 


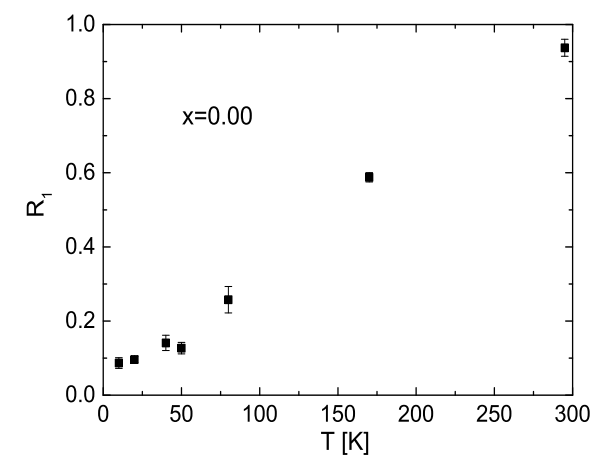

Figure 5.9: Temperature dependence of the anisotropy ratio, $R_{1}$, of the spin lattice relaxation rates.

\subsubsection{Temperature dependence of the spin-spin relax- ation in $\mathrm{CeFeAsO}$}

Spin-spin relaxation times were all measured with a 2D-Hahn-echo-sequence, as described on page 49. Typical values for $T_{2}$ are around $1200 \mu$ s for $a b \| B_{0}$ and lower for $c \| B_{0}$. Therefore, the values for $\tau_{\text {echo }}$ where varied in a range between $10 \mu \mathrm{s}$ to $1500 \mu \mathrm{s} 2$. A typical measurement for $\mathrm{x}=0.00$ is shown in figure 5.10, with a Lorentz fit from equation 2.20. All other measurements for the pure compound could also be fitted with a Lorentzian decay and the $T_{2}$ values are plotted in figure 5.11a. The spin-spin relaxation rate, $1 / T_{2}$, in figure $5.11 \mathrm{~b}$ shows the same trend as $1 / T_{1}$. The anisotropy ratio, $R_{2}$, is shown in figure 5.11c , reveals a similar trend as $R_{1}$, towards higher anisotropy with decreasing temperature down to a ratio of 0.1 at $10 \mathrm{~K}$. The values of $T_{2}$ rapidly increase (double) for $10 \mathrm{~K}$, because with $10 \mathrm{~K}$ we reach the vicinity of the ordering temperature from Ce moments [2]. This slows down the Ce fluctuations and therefore reduce all connected relaxations rates, which leads to the observed increase in the spin-spin relaxation time.

\footnotetext{
${ }^{2}$ This results in separation times, $t$, in the range of $20 \mu \mathrm{s}$ to $3000 \mu \mathrm{s}$
} 


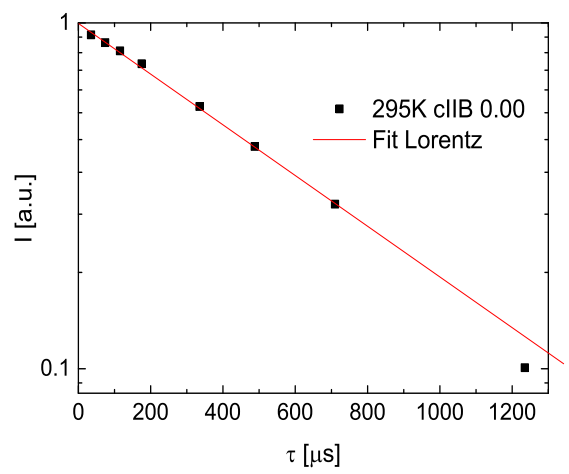

Figure 5.10: Integrated intensity of the echo after different $t$ for $T_{2}$ at $295 \mathrm{~K}$ for $c \| B_{0}$. Also included is a fit to a Lorentz decay function (equation 2.20).

\begin{tabular}{|c|c|c|}
\hline Temperature $[\mathrm{K}]$ & $T_{2}^{c}[\mu \mathrm{s}]$ & $T_{2}^{a b}[\mu \mathrm{s}]$ \\
\hline \hline 295 & $1180(40)$ & $1320(40)$ \\
\hline 170 & $740(20)$ & $1160(20)$ \\
\hline 100 & $540(50)$ & $1240(90)$ \\
\hline 80 & $340(20)$ & $1260(100)$ \\
\hline 50 & $230(20)$ & $1100(80)$ \\
\hline 40 & $200(10)$ & $1200(30)$ \\
\hline 20 & $185(25)$ & $1300(75)$ \\
\hline 10 & $340(20)$ & $2670(80)$ \\
\hline
\end{tabular}

Table 5.3: $T_{2}$ for CeFeAsO at different temperatures and both orientations and their experimental uncertainty. 


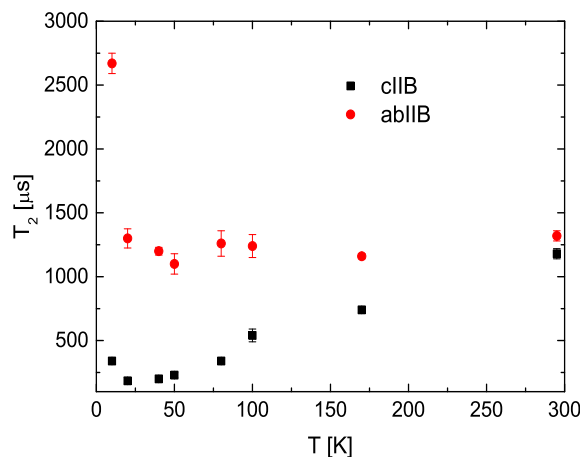

(a) $T_{2}$

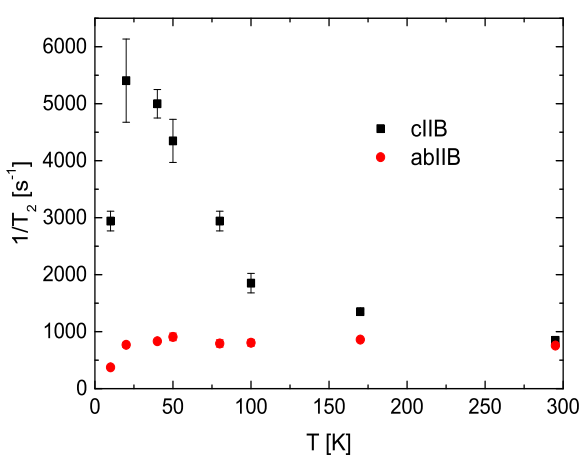

(b) $1 / T_{2}$

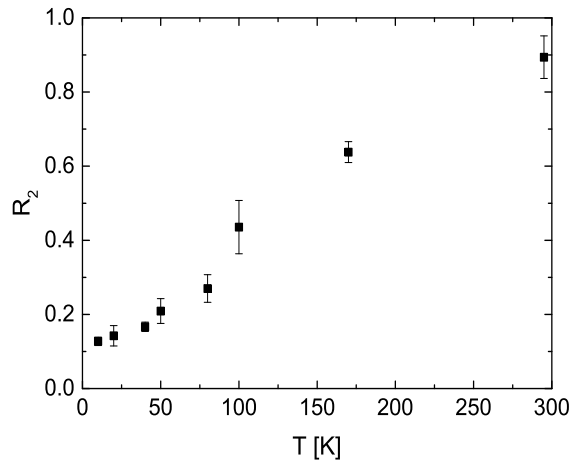

(c) $R_{2}$

Figure 5.11: Temperature dependence of the spin-spin relaxation time, rate and anisotropy ratio for $\mathrm{CeFeAsO}$ in both magnetic field directions. 


\subsection{NMR of $\mathrm{CeFeAsO}{ }_{0.93} \mathrm{~F}_{0.07}$}

The next sample in this study has a nominal F-doping of $\mathrm{x}=0.07$, which might be slightly in the superconducting region, $x>0.06$ [2]. However, no sign of superconductivity could be found, whereas a clear indication of a SDW transition between $10 \mathrm{~K}$ and $30 \mathrm{~K}$ is observed as shown below. The fact that SDW is nearly suppressed without a clear sign of superconductivity is an interesting prospect for the NMR measurements. The nominal doping values will be used for all samples throughout the study, since it is difficult to ascertain the true F-doping values of those compounds.

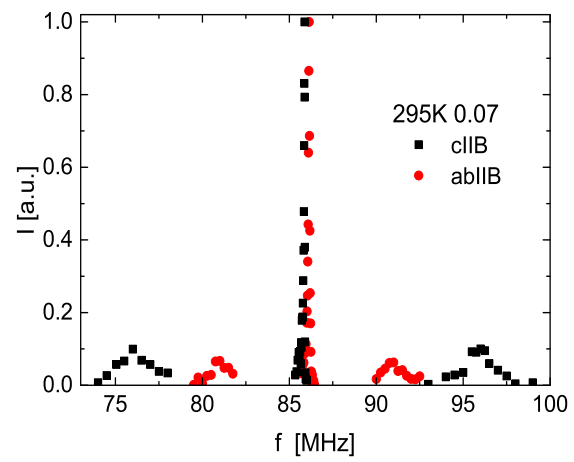

Figure 5.12: Room temperature spectra of $\mathrm{CeFeAsO}_{0.93} \mathrm{~F}_{0.07}$ for both orientations including their satellite transitions.

Figure 5.12 shows the room temperature spectra for both orientations, including their satellites. The quadrupole frequency, $f_{\mathrm{q}}$, can be determined from the frequency difference between the CT and the ST. This results in a quadrupole frequency for $c \| B_{0}, f_{\mathrm{q}}^{c}$, of $10.0(2) \mathrm{MHz}$ and for $a b \| B_{0}$, $\mathrm{f}_{\mathrm{q}}^{a b}$, of $5.0(2) \mathrm{MHz}$. Hence, $f_{\mathrm{q}}^{a b}$ is roughly half of $f_{\mathrm{q}}^{c}$, which could have been expected from the electric field gradient $\mathrm{S}^{3}$ (EFG).

\footnotetext{
${ }^{3}$ The diagonal elements of the EFG-tensor in the principal axis system have to fulfill the Laplace-equation, $V_{x x}+V_{y y}+V_{z z}=0$. Additional the asymmetry parameter was assumed to be 0 , which means that $V_{x x}=V_{y y}$. Hence, to fulfill the Laplace-equation these components have to be of same strength and opposite direction, $2 * V_{x x}=V_{z z}$.
} 


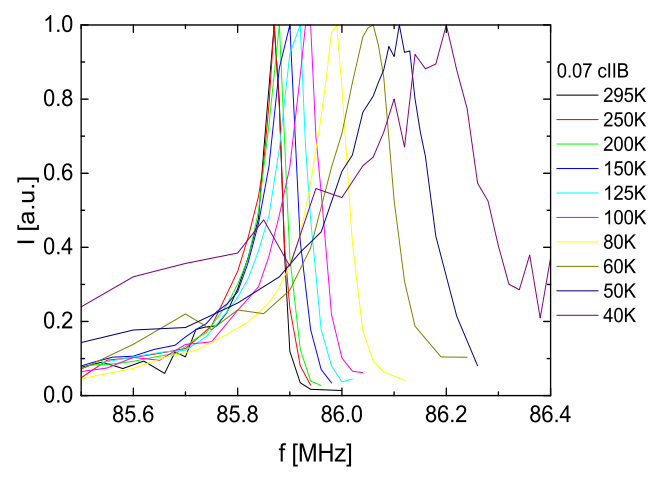

(a) $c \| B_{0}$

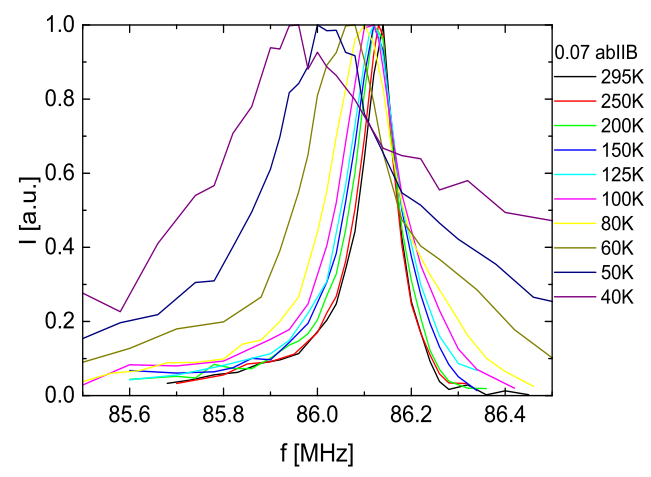

(b) $a b \| B_{0}$

Figure 5.13: Temperature dependence of the CT for temperatures from room temperature to $40 \mathrm{~K}$.

Figure 5.13 shows the temperature dependence of the CT line for both orientations from room temperature down to $40 \mathrm{~K}$. No magnetic ordering or transition can be seen down to $40 \mathrm{~K}$ in either direction, but there is a shift towards lower (higher) frequencies for $a b \| B_{0}\left(c \| B_{0}\right)$. The low temperature spectra is shown in figure 5.14 at $10 \mathrm{~K}$, where it can be seen that for $c \| B_{0}$ there are additional peaks that indicate that there is a SDW order at this temperature. There is no splitting for the $a b \| B_{0}$ orientation observed as it is expected within the hyperfine coupling scenario [184, 185]. From the spectra at higher temperatures it is possible to estimate that the SDW ordering temperature is around $20 \mathrm{~K}$. SDW ordering is not expected for $\mathrm{x}=0.07$ and this suggests that $\mathrm{x}$ is slightly lower and probably around $\mathrm{x}=0.06$. 


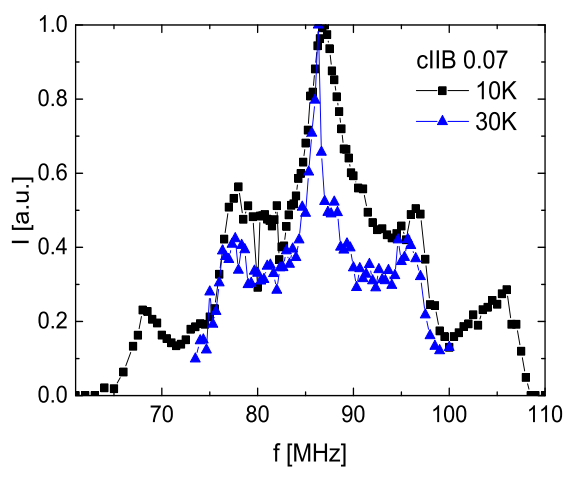

(a) $c \| B_{0}$

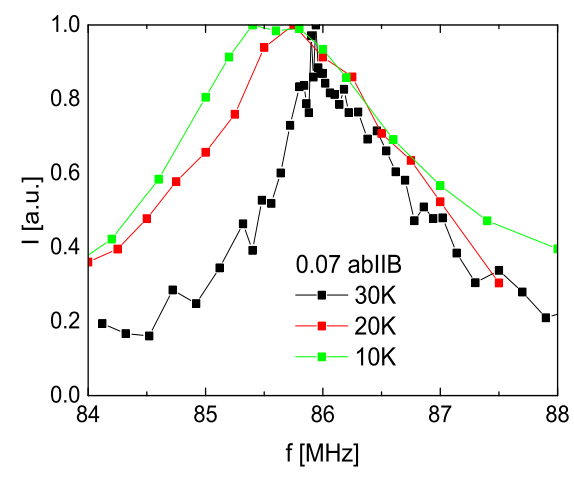

(b) $a b \| B_{0}$

Figure 5.14: Temperature dependence of the CT for temperatures $30 \mathrm{~K}$ to $10 \mathrm{~K}$ in both orientations.

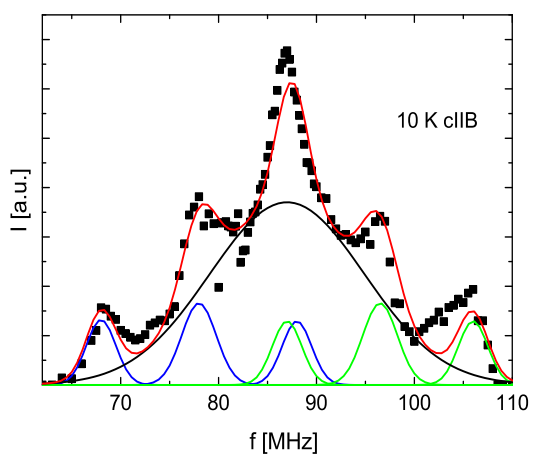

Figure 5.15: Spectra at $10 \mathrm{~K}$ for $c \| B_{0}$ with a fit to 2 sets of $3{ }^{75} \mathrm{As}$ NMR lines (green and blue) as well as a large Gaussian background (black). The cumulative fit is in red.

It is apparent in figure 5.15 that there is a broad background underneath the SDW split peaks. This may suggest that there might be a distribution of $\mathrm{x}$ where some regions correspond to SDW and other regions with higher $\mathrm{x}$ slightly above the critical point of $\mathrm{x}=0.06$ where there is no SDW. This can be illustrated in figure 5.15 where the data has been modeled with Gaussians to represented the SDW split peaks and a much broader Gaussian to represent the background from a distribution in fluorine doping where $\mathrm{x}$ is greater 
than 0.06. Based on earlier studies [2] of the change in the SDW ordering temperature with $\mathrm{x}$, it is possible to use the SDW ordering temperature of $20 \mathrm{~K}$ to estimate that the lower bound for $\mathrm{x}$ is 0.055 .

\subsubsection{NMR shift}

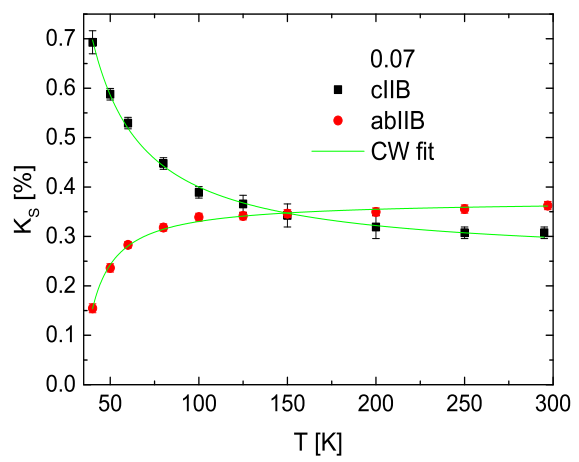

Figure 5.16: Magnetic shift for both orientations with a fit to Curie-Weiss (CW) temperature dependent behavior.

The magnetic shift can be calculated from equation 2.16 for $c \| B_{0}$ and 2.17 for $a b \| B_{0}$, which are shown in figure 5.16. Figure 5.16 shows the shift with a Curie-Weiss-like temperature dependent behavior above the SDW transition. Only FeAs and Ce have significant spin susceptibility in this material and equation (2.18) can be rewritten to:

$$
K_{\|, \perp}(T)=K_{\mathrm{L}, \|, \perp}+A_{\|, \perp}^{\mathrm{FeAs}} \chi_{\mathrm{FeAs}}(T)+A_{\|, \perp}^{\mathrm{Ce}} \chi_{\mathrm{Ce}}(T)
$$

Hence, $K_{\mathrm{S}}$ was replaced with the appropriate susceptibilities of the itinerant electrons of the FeAs-layer and of the localized Ce moments. Measurements of the magnetic shift of La1111, which does not possess a magnetic rare earth ion, has a temperature dependence of $K_{\mathrm{S}}$, that is over a magnitude smaller [160, 186] than values measured here. Only the spin susceptibility of FeAs, $\chi_{\mathrm{FeAs}}$, is relevant in this case. It is reasonable to assume that the other members of the 1111-family have similar values for $\chi_{\mathrm{FeAs}}$ and that it can be neglected in comparison to the larger contribution from the spin susceptibility 
of the rare-earth ion. Since $\chi_{\mathrm{Ce}}$ has a $\mathrm{CW}$ temperature dependent behavior equation (5.2) can be further rewritten to:

$$
K_{\|, \perp}(T)=K_{\mathrm{L}, \|, \perp}+\frac{b_{\|, \perp}}{T-\Theta}
$$

The NMR shifts in figure 5.16 were fitted using equation 5.3. The fitting parameters are shown in table 5.4. There are several things to note here. $\Theta$ is the same for both orientations within the experimental errors. This should be the case because the ordering of Ce moments is independent of the orientation of the sample. Zhao et al. [2] measured a very low antiferromagnetic ordering temperature for Ce, which is below $2 \mathrm{~K}$ at the critical point. Furthermore, the parameter $\mathrm{b}$ includes the hyperfine coupling constant and all constants from the temperature dependent part of $\chi_{\mathrm{s}}$. Because of this $b$ fulfills the Laplace-equation ${ }^{4}$, meaning $b_{c}=-2 \cdot b_{a b}$ within the experimental errors. The fitted values for the parameter $a$, which is the orbital shift, are different for both orientations through their orientation dependence.

\begin{tabular}{|c|c|c|c|}
\hline orientation & $\Theta[\mathrm{K}]$ & $K_{\mathrm{L}}[\%]$ & $\mathrm{b}[\% \mathrm{~K}]$ \\
\hline \hline$c \| B_{0}$ & $-1(2)$ & $0.23(1)$ & $18(2)$ \\
\hline$a b \| B_{0}$ & $-2(2)$ & $0.40(1)$ & $-8(2)$ \\
\hline
\end{tabular}

Table 5.4: Obtained fit-parameters from a CW fit to the NMR shift data.

\subsubsection{NMR linewidth}

The extracted NMR linewidths are plotted in figure 5.17 and were fitted to a CW-temperature dependent behavior, which fits well above $60 \mathrm{~K}$ (the fit parameters are shown in table 5.5) . This was done because the NMR linewidth should be dominated by magnetic interactions with Ce. The Curie temperatures are the same as those found for the NMR shift data within the experimental uncertainty.

\footnotetext{
${ }^{4}$ similar to the diagonal elements of the EFG in the principal axis system
} 


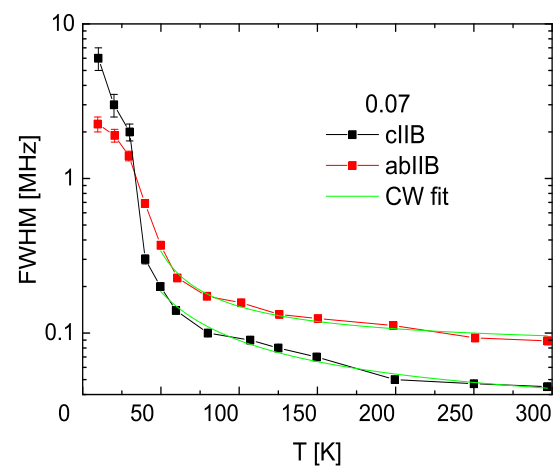

Figure 5.17: Temperature dependence of the linewidth for both orientations.

\begin{tabular}{|c|c|c|c|}
\hline orientation & $\Theta[\mathrm{K}]$ & $\mathrm{a}[\%]$ & $\mathrm{b}[\% \mathrm{~K}]$ \\
\hline \hline$c \| B_{0}$ & $-2(1)$ & $20(3)$ & $7200(600)$ \\
\hline$a b \| B_{0}$ & $-2(1)$ & $57(5)$ & $10100(600)$ \\
\hline
\end{tabular}

Table 5.5: Obtained fit parameters from the CW fit to the linewidth.

\subsubsection{Temperature dependence of the spin-lattice re- laxation of $\mathrm{CeFeAsO}_{0.93} \mathrm{~F}_{0.07}$}

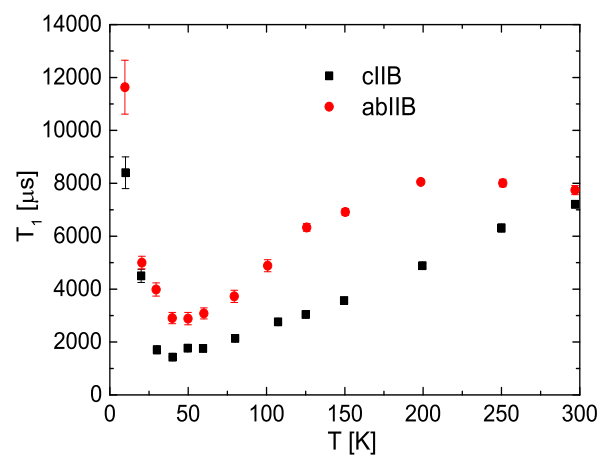

(a) $T_{1}$

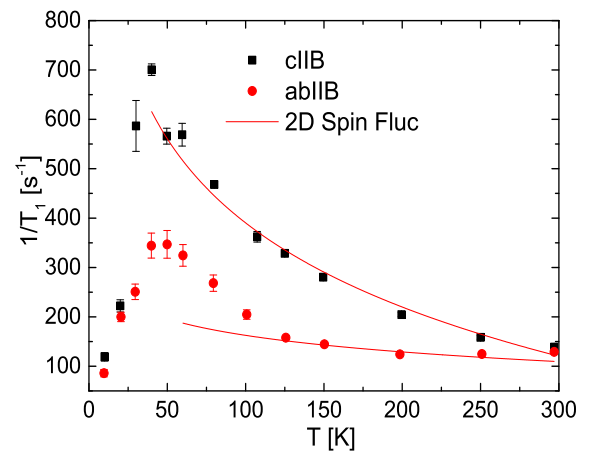

(b) $1 / \mathrm{T}_{1}$

Figure 5.18: Temperature dependence of the spin-lattice relaxation in $\mathrm{CeFeAsO}_{0.93} \mathrm{~F}_{0.07}$. 


\begin{tabular}{|c|c|c|}
\hline Orientation & $\mathrm{C}\left[\mathrm{s}^{-1}\right]$ & $\mathrm{T}_{0}[\mathrm{~K}]$ \\
\hline \hline$c \| B_{0}$ & $250(20)$ & $490(30)$ \\
\hline$a b \| B_{0}$ & $50(20)$ & $3000(3000)$ \\
\hline
\end{tabular}

Table 5.6: Fitted parameters from a 2D spin fluctuations fit to the data in figure $5.18 \mathrm{~b}$.

The measurements for the spin-lattice relaxation were done for different temperatures in both orientations and the results are presented in figure 5.18a. The temperature dependence of $1 / T_{1}$ is shown in figure $5.18 \mathrm{~b}$ where it increases up to a maximum around $50 \mathrm{~K}$, which is slightly above the observed SDW transition (see section 5.3). Values of $1 / T_{1}$ are compared with those from La1111 [180, 182, 187] and they are significant larger. Similar increases are observed for Pr1111, Nd1111, Sm1111 [7, 188] and SmCoPO [189]. This is an indication that the low-energy excitations from the $f$-electrons of the rare-earth elements $\mathrm{Ce}, \mathrm{Sm}, \mathrm{Nd}$ and $\mathrm{Pr}$ are the main probe of the As nuclei. It appears that the temperature dependence of $1 / T_{1}$ above the SDW transition can be described by Moriya's theory [49] for weakly itinerant 2D AFM such as in Sm1111 [188]. Already the fit to the linewidth and the Knight shift indicated an AFM ordering at very low temperatures and it is known through neutron diffraction experiments that Ce moments order AFM below $2 \mathrm{~K}$ [2] for a doping of $\mathrm{x}=0.07$. When the spin fluctuations are the main relaxation mechanism, is the spin-lattice relaxation given by [190]:

$$
\frac{1}{T_{1}}=\frac{2 \gamma^{2} T}{N_{0}} \sum_{q}\left|A_{\mathrm{q}}\right|^{2} \frac{\operatorname{Im} \chi_{\perp}\left(q, \omega_{0}\right)}{\omega_{0}}
$$

where $N_{0}$ is the number of magnetic atoms per unit volume, $A_{q}$ is the Fourier q-component of the hyperfine coupling constant and $\chi_{\perp}\left(q, \omega_{0}\right)$ is the transverse component of the dynamical spin susceptibility at the resonance frequency $\omega_{0}$. For AFM correlations it is predicted [191, 192] that 3D fluctuations should be $\propto\left(\frac{T}{T_{0}}\right)^{\frac{1}{4}}$ and $2 \mathrm{D}$ fluctuations $\propto-\ln \left(\frac{T}{T_{0}}\right) . T_{0}$ comes from the spin fluctuation theory [191] and is a characteristic parameter thereof. Good fits were obtained for the $2 \mathrm{D}$ case, shown in figure $5.18 \mathrm{~b}$ as solid lines. The parameters obtained from the fit are shown in table 5.6. 
The anisotropy ratio (equation 5.1) is plotted in figure 5.19 and shows first a decrease in the anisotropy ratio with decreasing temperature until $125 \mathrm{~K}$. Below this temperature no further change is observed due to an arising influence from spin fluctuations of the SDW transition after which another decrease is observed, because the former spin fluctuations are gone again.

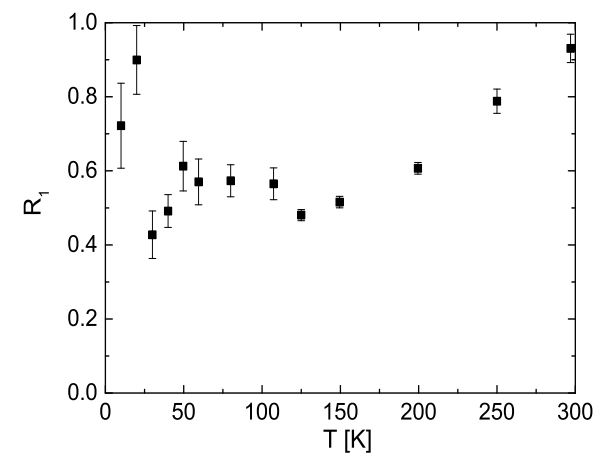

Figure 5.19: Temperature dependence of the anisotropy ratio $R_{1}$ for $\mathrm{CeFeAsO}_{0.93} \mathrm{~F}_{0.07}$.

\subsubsection{Temperature dependence of the spin-spin relaxation in $\mathrm{CeFeAsO}_{0.93} \mathrm{~F}_{0.07}$}

The spin-spin relaxation time, $T_{2}$, at room temperature is around $1530 \mu \mathrm{s}$ for $a b \| B_{0}$ and $1370 \mu \mathrm{s}$ for $c \| B_{0}$. With decreasing temperature these values become lower with the exception at $10 \mathrm{~K}$ as shown in table 5.7 and plotted in figure 5.20 . 


\begin{tabular}{|c|c|c|c|c|}
\hline Temperature $[\mathrm{K}]$ & $\mathrm{T}_{2}^{c}[\mu \mathrm{s}]$ & $\mathrm{T}_{2, \text { short }}^{c}[\mu \mathrm{s}]$ & $\mathrm{T}_{2}^{a b}[\mu \mathrm{s}]$ & $\mathrm{T}_{2, \text { short }}^{a b}[\mu \mathrm{s}]$ \\
\hline \hline 295 & $1370(50)$ & & $1530(50)$ & \\
\hline 250 & $1300(80)$ & & $1540(50)$ & \\
\hline 200 & $990(30)$ & & $1490(40)$ & \\
\hline 150 & $740(20)$ & & $1360(30)$ & \\
\hline 125 & $625(10)$ & & $1250(20)$ & \\
\hline 107 & $550(10)$ & & $1025(25)$ & \\
\hline 80 & $415(5)$ & & $915(40)$ & $80(45)$ \\
\hline 60 & $540(90)$ & $185(35)$ & $895(40)$ & $155(40)$ \\
\hline 50 & $390(50)$ & $85(20)$ & $910(60)$ & $140(40)$ \\
\hline 40 & $350(110)$ & $90(60)$ & $950(50)$ & $190(30)$ \\
\hline 30 & $300(100)$ & $60(90)$ & $820(30)$ & $80(15)$ \\
\hline 20 & $350(120)$ & $50(60)$ & $850(45)$ & $90(30)$ \\
\hline 10 & $340(110)$ & $135(145)$ & $1410(30)$ & $170(45)$ \\
\hline
\end{tabular}

Table 5.7: Two components of $T_{2}$ for $\mathrm{CeFeAsO}_{0.93} \mathrm{~F}_{0.07}$ at different temperatures and orientations including their uncertainty (in brackets).

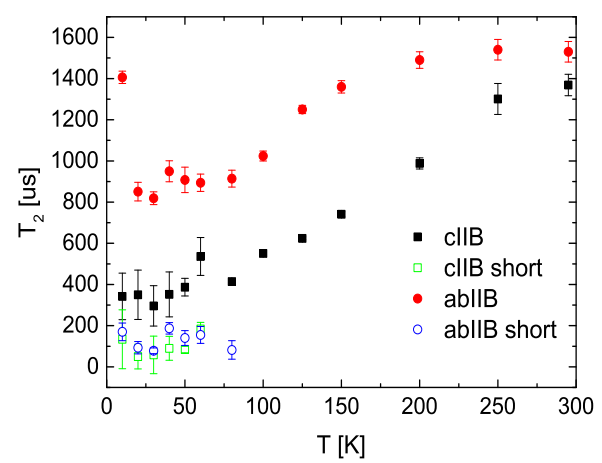

Figure 5.20: Temperature dependence of $T_{2}$ for both orientations and both components.

Contrary to $\mathrm{x}=0.00 T_{2}$ cannot be fitted over the whole range with a single Lorentzian component. An example for both regions and in both directions is shown in figure 5.21. $1 / T_{2}$ increases until the second component appears, after which it becomes stable as shown in figure 5.22a. This double component behavior appears around the temperature where $R_{1}$ becomes flat. Therefore, the second (short) component is most likely due to the appearance 
of iron spin fluctuations. A similar anisotropy behavior as seen in $R_{1}$ is also seen for $R_{2}$ as shown in figure $5.22 \mathrm{~b}$.

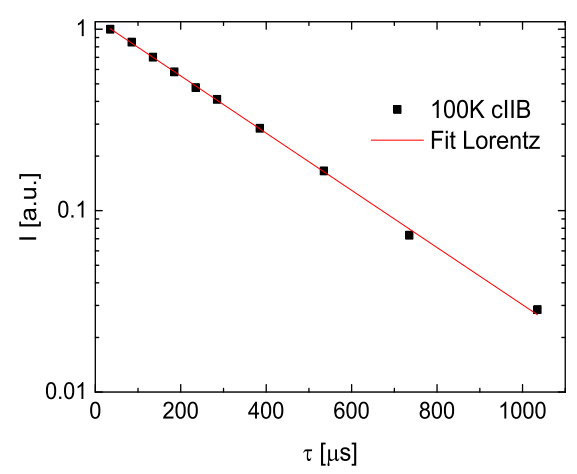

(a) $100 \mathrm{~K}$ in $c \| B_{0}$ with a single component

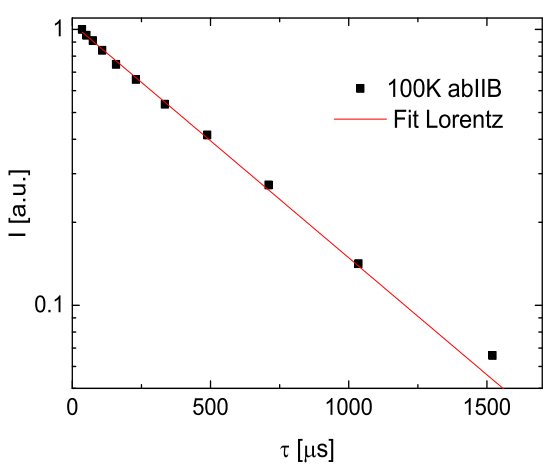

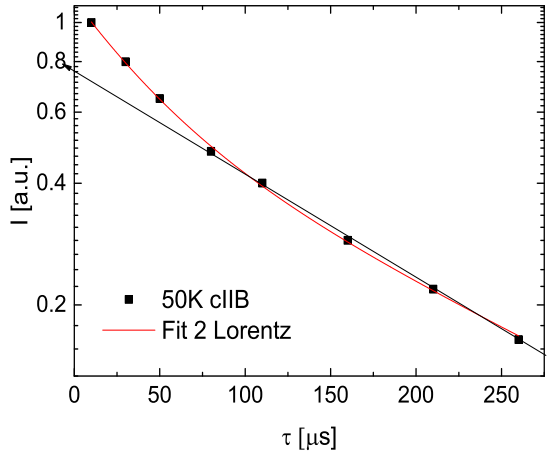

(b) $50 \mathrm{~K}$ in $c \| B_{0}$ with a double component

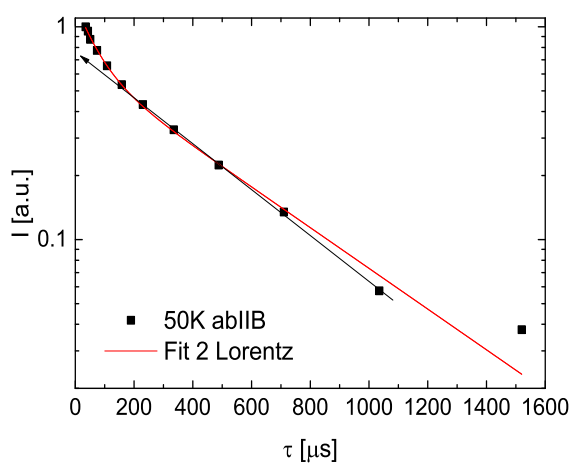

(c) $100 \mathrm{~K}$ in $a b \| B_{0}$ with a single component (d) $50 \mathrm{~K}$ in $a b \| B_{0}$ with a double component

Figure 5.21: Fits to $T_{2}$ measurements with single or double Lorentzian components. 


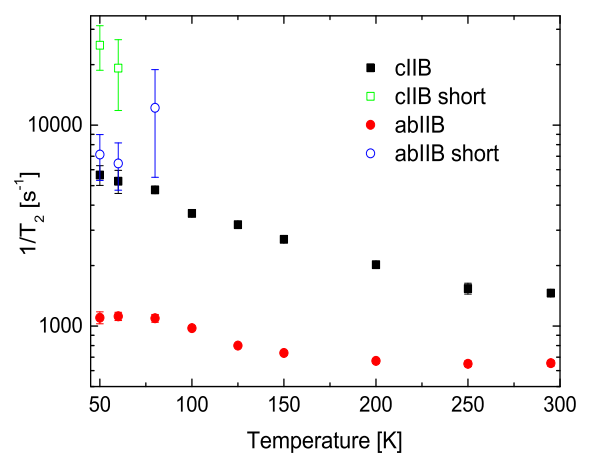

(a) $1 / T_{2}$

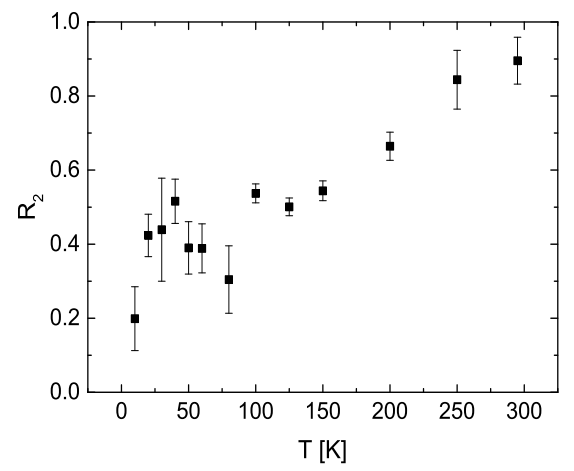

(b) $R_{2}$

Figure 5.22: The temperature dependence of the spin-spin relaxation rate and the anisotropy ratio. 


\subsection{NMR on $\mathrm{CeFeAsO}_{0.87} \mathrm{~F}_{0.13}$}

The sample with a nominal $\mathrm{F}$ doping of 0.13 has a $T_{\mathrm{C}}$ of $35 \mathrm{~K}$. Therefore it is positioned on the underdoped region of the phase diagram.

\subsubsection{Temperature dependence of ${ }^{75} \mathrm{As}$ NMR spectra}

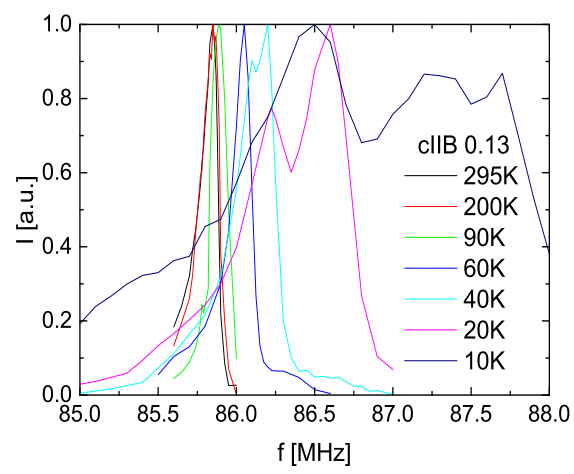

(a) $c \| B_{0}$

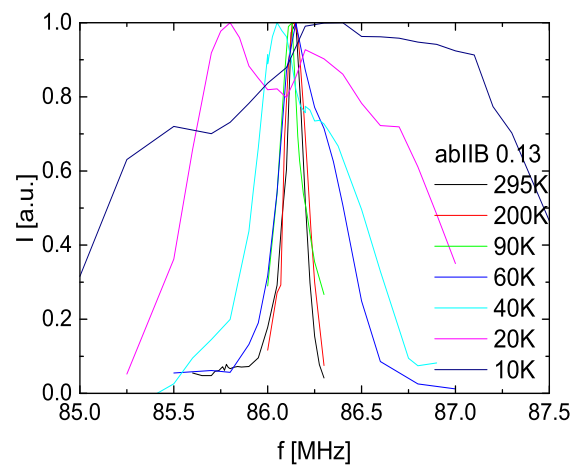

(b) $a b \| B_{0}$

Figure 5.23: Temperature dependence of the central transition for $\mathrm{x}=0.13$ for both magnetic field orientations.

Since this sample is superconducting, we do not expect to see a splitting in the central-lines originating from a SDW transition. A splitting is however observed at $40 \mathrm{~K}$ and below for $c \| B_{0}$ (figure 5.23) and it occurs at $60 \mathrm{~K}$ for $a b \| B_{0}$ (figure 5.23). These splittings (more than two peaks) come from 
charge inhomogeneities due to an inhomogeneous doping environment [193]. A possible alternative explanation in terms of vortices could be excluded because this would limit such a splitting to only two lines (one from inside and outside of the vortices) [193. However, a third line was observed at even lower temperature, which is noticeable at $10 \mathrm{~K}$ in both directions in figure 5.23

\section{NMR shift}

The magnetic shift is shown in figure 5.24 and the shift of the CT peaks can be fitted to a $\mathrm{CW}$ temperature dependent behavior. The main peaks have a $\mathrm{CW}$ temperature of $0 \mathrm{~K}$ and the secondary lines have negative values, which are shown in table 5.8. This is an indication that the Knight shift is still dominated by interactions with Ce moments.

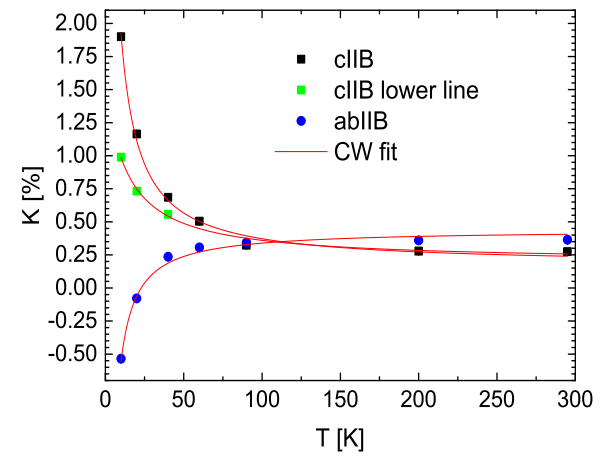

Figure 5.24: Temperature dependence of the NMR shift for both magnetic field orientations.

\begin{tabular}{|c|c|c|c|}
\hline orientation & $\Theta[\mathrm{K}]$ & $K_{\mathrm{L}}[\%]$ & $\mathrm{b}[\% \mathrm{~K}]$ \\
\hline \hline$c \| B_{0}$ & $-1(2)$ & $0.17(4)$ & $20(4)$ \\
\hline$c \| B_{0}$ lower line & $-13(5)$ & $0.20(4)$ & $18(5)$ \\
\hline$a b \| B_{0}$ & $0(2)$ & $0.44(4)$ & $-10(2)$ \\
\hline
\end{tabular}

Table 5.8: CW fit parameters for the temperature dependence of the NMR shift for $\mathrm{x}=0.13$. 


\subsubsection{Temperature dependence of the spin-lattice relaxation in $\mathrm{CeFeAsO}_{0.87} \mathrm{~F}_{0.13}$}

The spin-lattice-relaxation times are plotted in figure 5.25a and the rates in figure $5.25 \mathrm{~b}$. The rate peaks around $T_{C}$ and decreases afterwards due to the transition into the superconducting state.

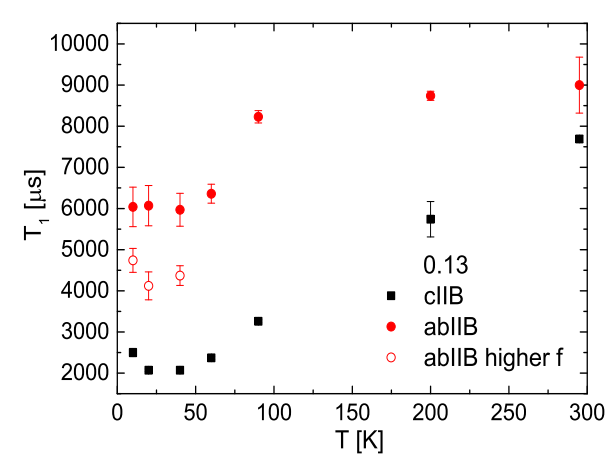

(a) $T_{1}$

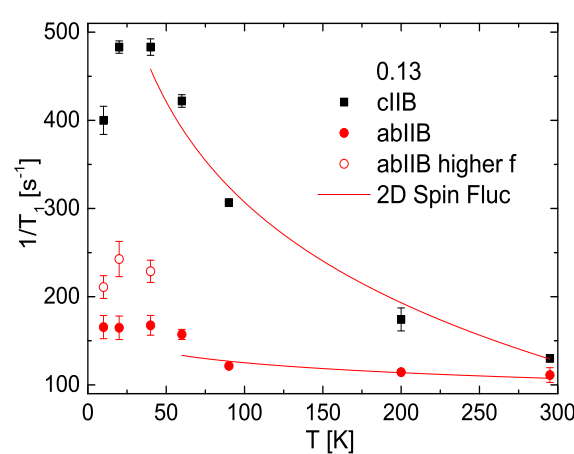

(b) $1 / T_{1}$

Figure 5.25: Temperature dependence of the spin-lattice relaxation for both magnetic field orientations.

The high temperature $1 / T_{1}$ data can be fitted to a $2 \mathrm{D}$ fluctuations model (section 5.3.3) similar to $\mathrm{x}=0.07$ with fitting parameters shown in table 5.9 . The anisotropy ratio $R_{1}$ (figure 5.26) shows a strong temperature dependent behavior until $100 \mathrm{~K}$, after which it stabilizes at a value of 0.4 for low temperatures.

\begin{tabular}{|c|c|c|}
\hline sample & $\mathrm{C}\left[\mathrm{s}^{-1}\right]$ & $\mathrm{T}_{0}[\mathrm{~K}]$ \\
\hline \hline $\mathrm{c} \| \mathrm{B}$ & $180(20)$ & $580(60)$ \\
\hline $\mathrm{ab} \| \mathrm{B}$ & $22(13)$ & $36000(11000)$ \\
\hline
\end{tabular}

Table 5.9: Parameters from fitting the data of figure 5.25b to $2 \mathrm{D}$ spin fluctuations according to equation 5.1 . 


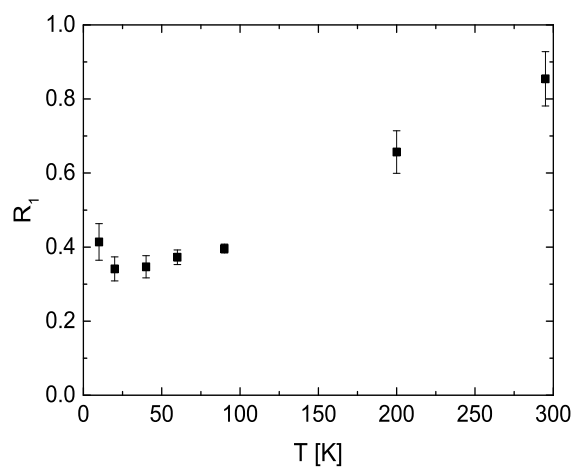

Figure 5.26: Temperature dependence of anisotropy ratio, $R_{1}$.

\subsubsection{Temperature dependence of the spin-spin relaxation in $\mathrm{CeFeAsO}_{0.87} \mathrm{~F}_{0.13}$}

All the $T_{2}$ data could again be fitted to a single Lorentzian component. However, both observed central transition peaks in the spectra (figure 5.23) show slightly different values of $T_{2}$, which is around $1520 \mu \mathrm{s}$ for $a b \| B_{0}$ and $1305 \mu \mathrm{s}$ for $c \| B_{0}$ (figure 5.27) at room temperature. These values are close to the other values observed for lower F-doping and $T_{2}$ also decreases with temperature, which is shown in table 5.10 and plotted in figure 5.27 .

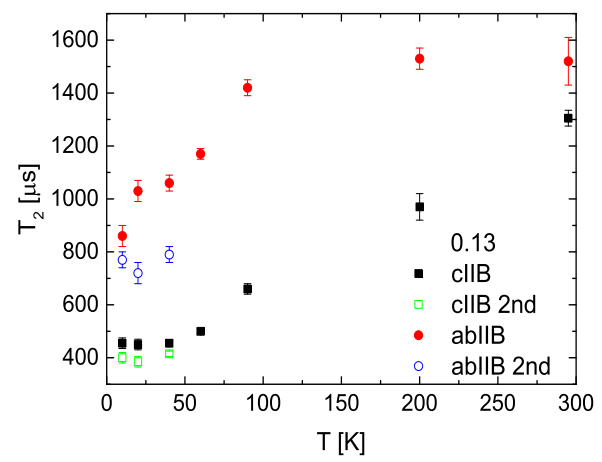

Figure 5.27: Temperature dependence of $T_{2}$ for both orientations and both components. 


\begin{tabular}{|c|c|c|}
\hline Temperature $[\mathrm{K}]$ & $\mathrm{T}_{2}^{c}[\mu \mathrm{s}]$ & $\mathrm{T}_{2}^{a b}[\mu \mathrm{s}]$ \\
\hline \hline 295 & $1310(30)$ & $1520(90)$ \\
\hline 200 & $970(50)$ & $1530(40)$ \\
\hline 90 & $660(20)$ & $1420(30)$ \\
\hline 60 & $500(10)$ & $1170(20)$ \\
\hline 40 & $455(10)$ & $1060(30)$ \\
\hline 20 & $450(20)$ & $1030(40)$ \\
\hline 10 & $455(20)$ & $860(40)$ \\
\hline
\end{tabular}

Table 5.10: Temperature dependence of $T_{2}$ for both orientations and both components.

A peak is clearly observed around $T_{\mathrm{C}}$ when $T_{2}$ is plotted as $1 / T_{2}$ as shown in figure 5.28a. The anisotropy ratio $R_{2}$ (figure 5.28b) displays a similar temperature dependent behavior to $R_{1}$, with a flat region at low temperature around a value of 0.5 .

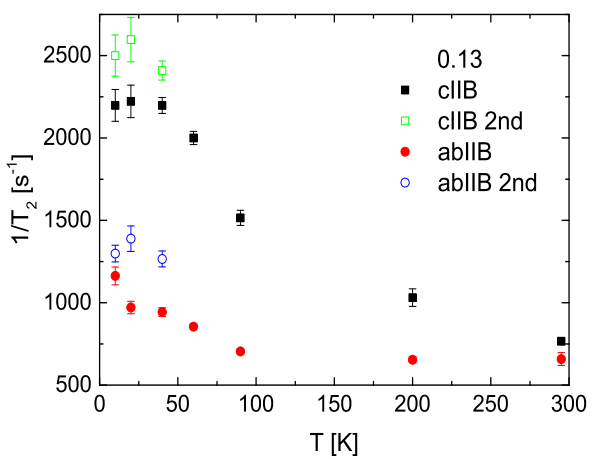

(a) $1 / T_{2}$

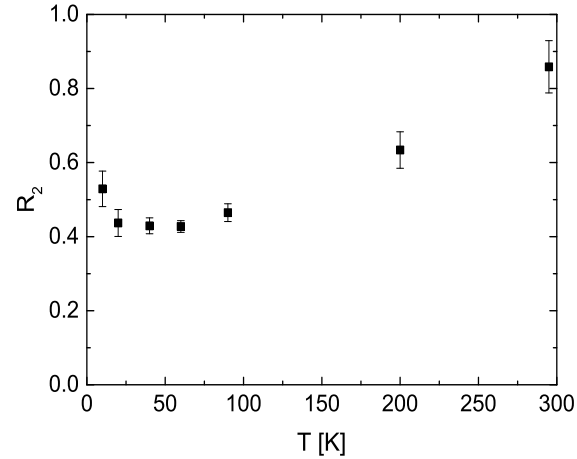

(b) $R_{2}$

Figure 5.28: Temperature dependence of the spin-spin relaxation rate and the anisotropy ratio for both magnetic field directions. 


\subsection{NMR on $\mathrm{CeFeAsO}{ }_{0.80} \mathrm{~F}_{0.20}$}

The sample with a nominal $\mathrm{F}$ doping of 0.20 is superconducting and in the overdoped region of the phase diagram [2]. The results from this doping have already been published by others in our group [193. Therefore, a brief description of the results will be presented here for comparison with other doping. The new measurement done in this thesis is on the spin-spin relaxation rate.

\subsubsection{Temperature dependence of the ${ }^{75}$ As NMR spec- tra}

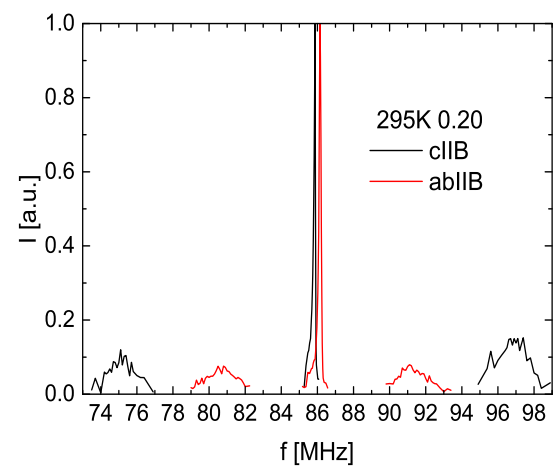

Figure 5.29: Room temperature spectra for both orientations. 


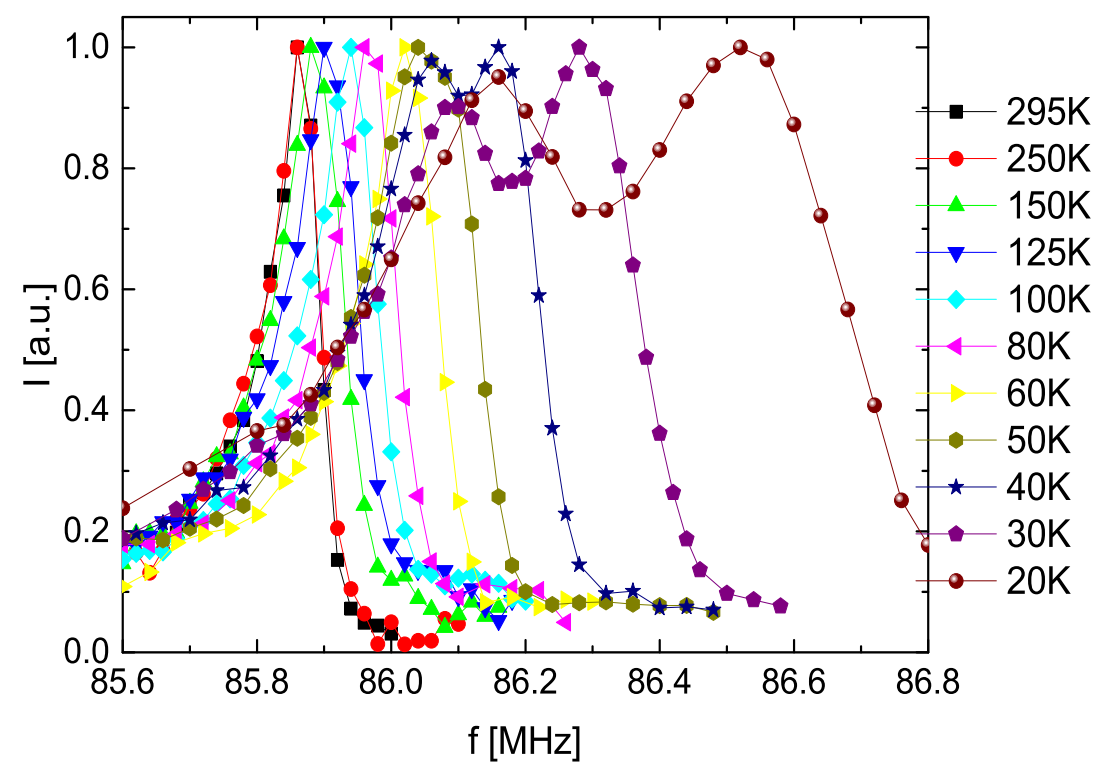

(a) $c \| B_{0}$

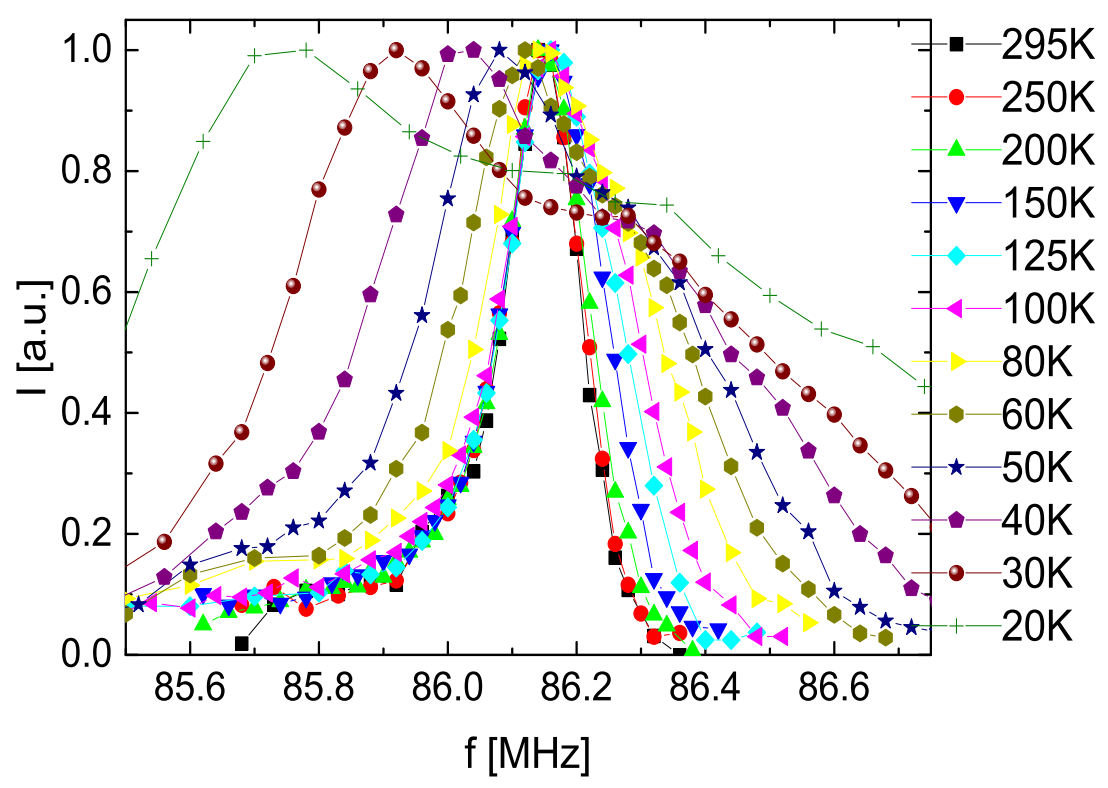

(b) $a b \| B_{0}$

Figure 5.30: Temperature dependence of the central transition of $\mathrm{x}=0.20$ for both magnetic field directions. 
The room temperature NMR spectra are plotted in figure 5.29. They are similar to the NMR spectra from $\mathrm{x}=0.13$ (figure ) where the CT and the satellites can be seen. The temperature dependent behavior is similar to the other superconducting sample with $\mathrm{x}=0.13$ as can be seen in figure 5.30 . There is also a low-temperature splitting into two lines, which occurs at a higher temperature than $\mathrm{x}=0.13$. For the underdoped compound the splitting could be observed at $60 \mathrm{~K}$ for $a b \| B_{0}$ and at $40 \mathrm{~K}$ for $c \| B_{0}$, whereas it can be seen here at $100 \mathrm{~K}$ for $a b \| B_{0}$ and at $50 \mathrm{~K}$ for $c \| B_{0}$. The reasons for the appearance have already been discussed for $\mathrm{x}=0.13$ (section 5.4) and in Rybicki et al. [193].

\section{NMR shift}

The temperature dependence of the NMR shift is shown in figure 5.31a. There is a trend to higher values for $c \| B_{0}$ and to lower for $a b \| B_{0}$ with decreasing temperature. Both directions can be fitted to a $\mathrm{CW}$ temperature dependent behavior as shown in figure 5.31. All the fitting parameters are shown in table 5.11 .

\begin{tabular}{|c|c|c|c|}
\hline orientation & $\Theta[\mathrm{K}]$ & $K_{\mathrm{L}}[\%]$ & $\mathrm{b}[\% \mathrm{~K}]$ \\
\hline \hline $\mathrm{c} \| \mathrm{B}$ & $-1(1)$ & $0.19(1)$ & $18(1)$ \\
\hline c $\|$ B lower f & $-40(10)$ & $0.18(2)$ & $25(6)$ \\
\hline ab\|B & $-1(2)$ & $0.40(2)$ & $-11(2)$ \\
\hline
\end{tabular}

Table 5.11: The obtained fitting parameters from CW fit of Knight shift. 


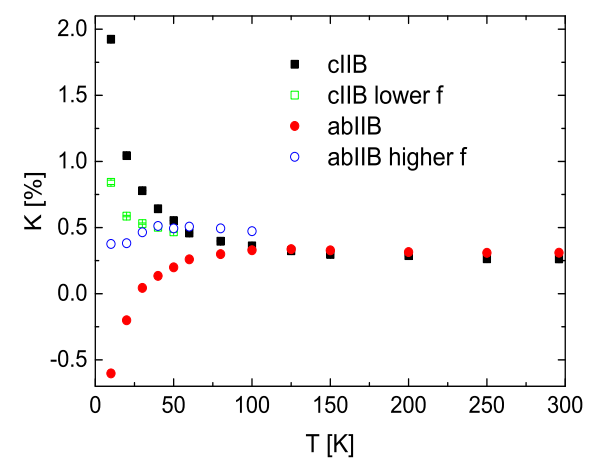

(a) $K$

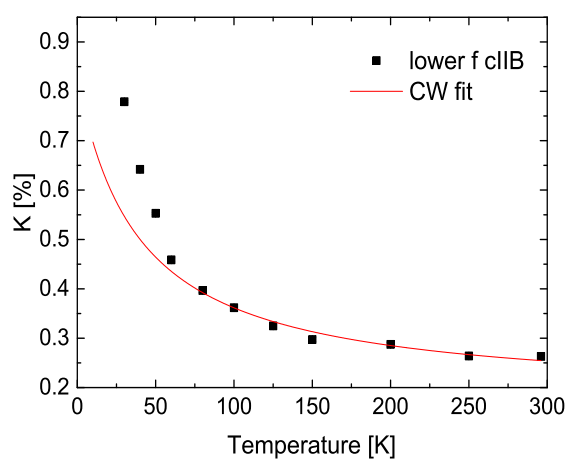

(c) Lower frequency peaks of $c \| B_{0}$

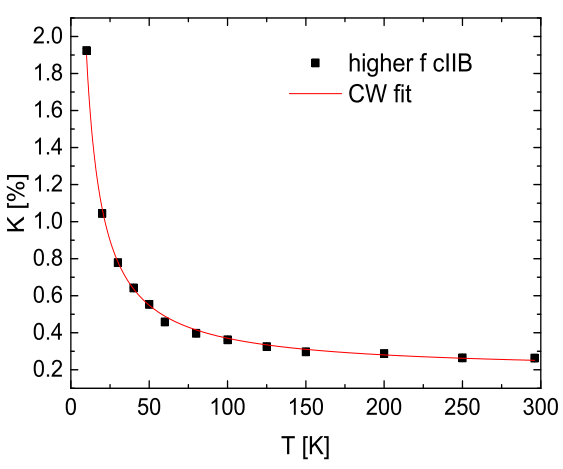

(b) Higher frequency peak of $c \| B_{0}$

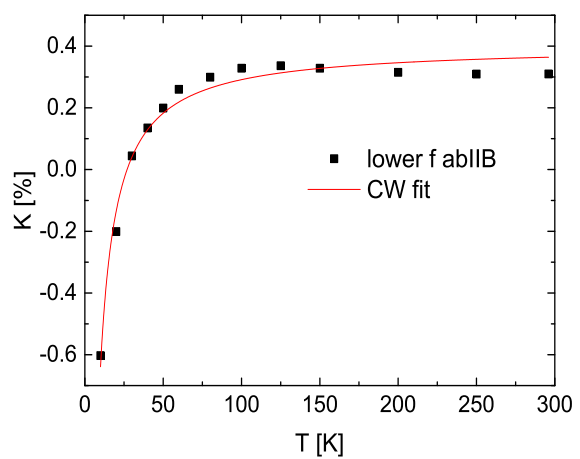

(d) Lower frequency peak of $a b \| B_{0}$

Figure 5.31: Temperature dependence of the magnetic shift in both magnetic field orientations and CW fits to the upper and lower frequency peaks.

\section{NMR linewidth}

The linewidth is plotted in figure 5.32 and was fitted to a CW temperature dependent behavior, which fits well above $60 \mathrm{~K}$. This was done, because the temperature dependence of the linewidth should be dominated by magnetic interaction with $\mathrm{Ce}$, whereas below $60 \mathrm{~K}$ superconductivity becomes important. The fitted CW temperature $\theta$ is $0 \mathrm{~K}$ within the experimental errors as shown in table 5.12, which indicates that there is no ordering of the $\mathrm{Ce}$ moments anymore at such high F-doping. 


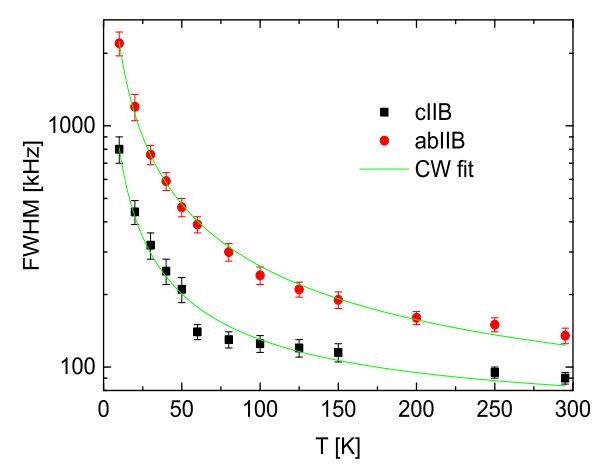

Figure 5.32: Temperature dependence of the NMR linewidth for both orientations.

\begin{tabular}{|c|c|c|c|}
\hline orientation & $\Theta[\mathrm{K}]$ & $\mathrm{a}[\mathrm{kHz}]$ & $\mathrm{b}[\mathrm{kHz} \mathrm{K}]$ \\
\hline \hline $\mathrm{c} \| \mathrm{B}$ & $0(2)$ & $60(10)$ & $7000(800)$ \\
\hline $\mathrm{ab} \| \mathrm{B}$ & $0(1)$ & $50(10)$ & $21000(1000)$ \\
\hline
\end{tabular}

Table 5.12: Obtained fitting parameters from a CW fit of the linewidth.

\subsubsection{Temperature dependence of the spin-lattice re- laxation in $\mathrm{CeFeAsO}_{0.80} \mathrm{~F}_{0.20}$}

Spin-lattice relaxation measurements were performed for several temperatures in both orientations and the results 193 are presented in table 5.13 and figure $5.33 \mathrm{a}$. 


\begin{tabular}{|c|c|c|}
\hline Temperature $[\mathrm{K}]$ & $T_{1}^{c}[\mathrm{\mu s}]$ & $T_{1}^{a b}[\mathrm{\mu s}]$ \\
\hline \hline 295 & $8000(250)$ & $8650(160)$ \\
\hline 250 & $6920(240)$ & $9140(130)$ \\
\hline 200 & $5790(390)$ & $8850(190)$ \\
\hline 155 & $4310(110)$ & $9050(270)$ \\
\hline 125 & $3590(50)$ & $8140(160)$ \\
\hline 100 & $3180(110)$ & $7530(260)$ \\
\hline 80 & $2770(50)$ & $7150(220)$ \\
\hline 60 & $2410(50)$ & $6560(330)$ \\
\hline 50 & $2400(50)$ & $6060(270)$ \\
\hline 40 & $2200(30)$ & $5610(280)$ \\
\hline 30 & $2130(40)$ & $5570(240)$ \\
\hline 20 & $2190(60)$ & $6120(450)$ \\
\hline 10 & $2620(90)$ & $8090(870)$ \\
\hline
\end{tabular}

Table 5.13: $\mathrm{T}_{1}$ for $\mathrm{CeFeAsO}_{0.80} \mathrm{~F}_{0.20}$ at different temperatures and both field orientations with their uncertainties (in brackets).

The spin-lattice relaxation rate was fitted to 2D spin fluctuations (equation 5.4 similar to $\mathrm{x}=0.07$ and $\mathrm{x}=0.13$. Results are shown in figure $5.33 \mathrm{~b}$ and fit parameters in table 5.14. Finally, the observed relaxation rates can be displayed in the anisotropy ratio $R_{1}$, which is shown in figure $5.33 \mathrm{c}$. 


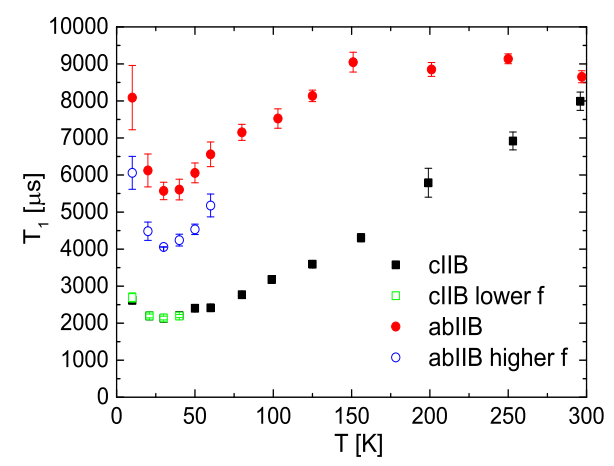

(a) $T_{1}$
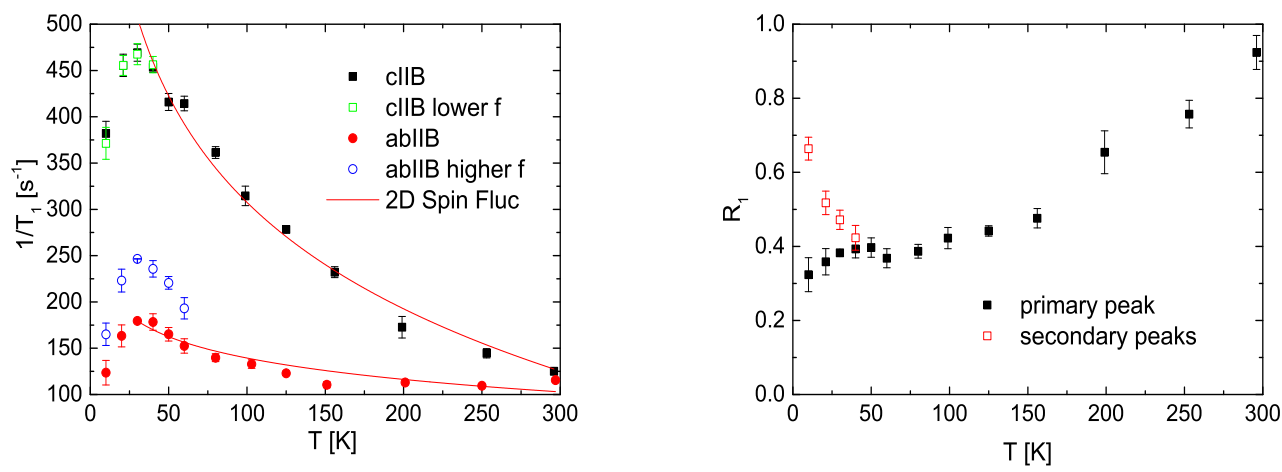

(b) $1 / T_{1}$ with fitting to $2 \mathrm{D}$ spin fluctuations

(c) $R_{1}$

Figure 5.33: Temperature dependence of the spin-lattice relaxation in both magnetic field orientations.

\begin{tabular}{|c|c|c|}
\hline orientation & $\mathrm{C}\left[\mathrm{s}^{-1}\right]$ & $\mathrm{T}_{0}[\mathrm{~K}]$ \\
\hline \hline $\mathrm{c} \| \mathrm{B}$ & $163(7)$ & $650(60)$ \\
\hline $\mathrm{ab} \| \mathrm{B}$ & $33(4)$ & $6100(3000)$ \\
\hline
\end{tabular}

Table 5.14: Fit parameters according to equation 5.4 from $1 / T_{1}$ data in figure $5.33 \mathrm{~b}$. 


\subsubsection{Temperature dependence of the spin-spin relaxation in $\mathrm{CeFeAsO}_{0.80} \mathrm{~F}_{0.20}$}

Spin-spin relaxation measurements were performed as described earlier in section 2.6 .3 and start with a value of $1570 \mu \mathrm{s}$ for both directions. These times decrease at a faster rate with decreasing temperature for $c \| B_{0}$ than for $a b \| B_{0}$, as shown in table 5.15 and in figure 5.35a. All temperatures and directions could be fitted with a single Lorentzian component, except at $10 \mathrm{~K}$ for $c \| B_{0}$, where a much better fit is obtained when using two Lorentzian components as shown in figure 5.34 . The spin-spin relaxation rate $1 / T_{2}$ peaks roughly around $T_{\mathrm{C}}$, as can be seen in figure $5.35 \mathrm{~b}$. The anisotropy ratio $R_{2}$ has a value of 1 from room temperature down to $200 \mathrm{~K}$ and then decreases with decreasing temperature reaching a value of 0.4 at the lowest measured temperature as shown in figure $5.35 \mathrm{c}$.

\begin{tabular}{|c|c|c|c|c|}
\hline Temperature $[\mu \mathrm{s}]$ & $T_{2}^{c}[\mathrm{\mu s}]$ & $T_{2}^{c, \text { lower }}[\mathrm{\mu s}]$ & $T_{2}^{a b}[\mu \mathrm{s}]$ & $T_{2}^{a b, \text { higher }}[\mu \mathrm{s}]$ \\
\hline \hline 295 & $1580(100)$ & & $1570(70)$ & \\
\hline 250 & $1570(150)$ & & $1600(90)$ & \\
\hline 200 & $1510(90)$ & & $1500(110)$ & \\
\hline 150 & $960(70)$ & & $1470(110)$ & \\
\hline 125 & $770(20)$ & & $1450(100)$ & \\
\hline 100 & $750(40)$ & & $1470(60)$ & \\
\hline 80 & & & $1300(20)$ & \\
\hline 60 & $530(20)$ & & $1180(30)$ & $1000(50)$ \\
\hline 50 & $490(20)$ & & $1120(30)$ & $830(30)$ \\
\hline 40 & $420(20)$ & $490(10)$ & $1050(30)$ & $800(30)$ \\
\hline 30 & $420(30)$ & $450(40)$ & $1030(40)$ & $750(30)$ \\
\hline 20 & $420(30)$ & $440(50)$ & $1080(50)$ & $740(40)$ \\
\hline 10 & $420(70)$ & $430(40)$ & & \\
\hline
\end{tabular}

Table 5.15: Temperature dependence of the spin-spin relaxation time, $T_{2}$. 


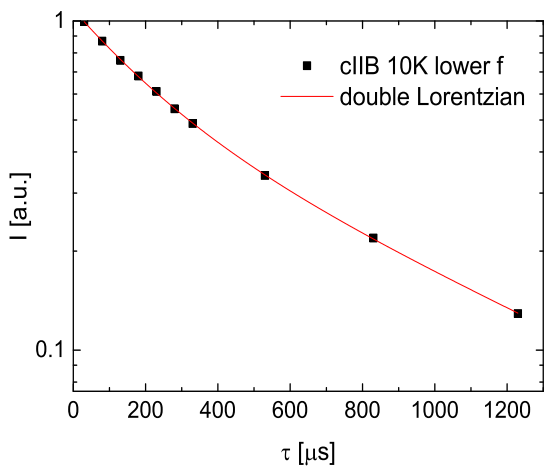

(a) Double component fit at $10 \mathrm{~K}$ for the lower frequency peak.

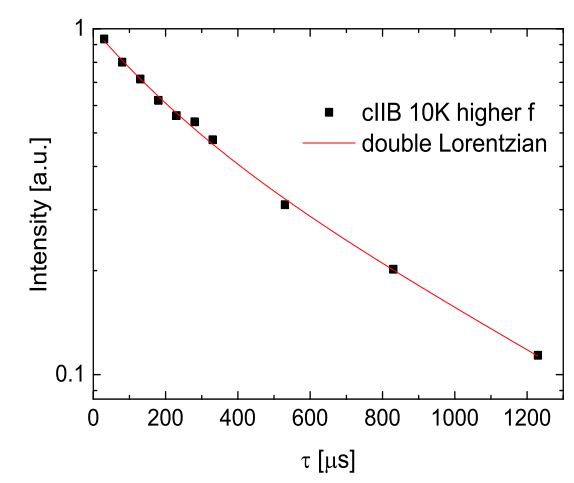

(c) Double component fit at $10 \mathrm{~K}$ for the higher frequency peak.

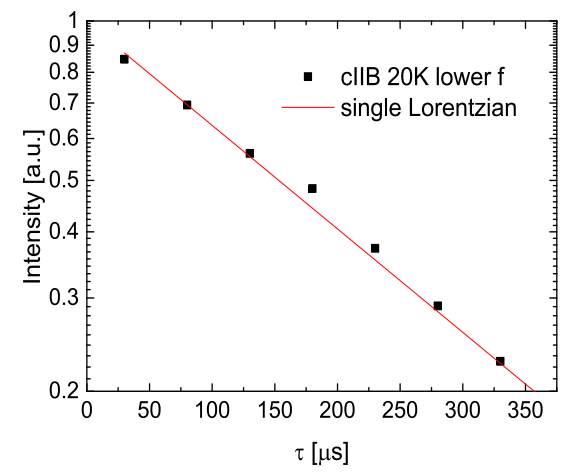

(b) Single component fit at $20 \mathrm{~K}$ for the lower frequency peak.

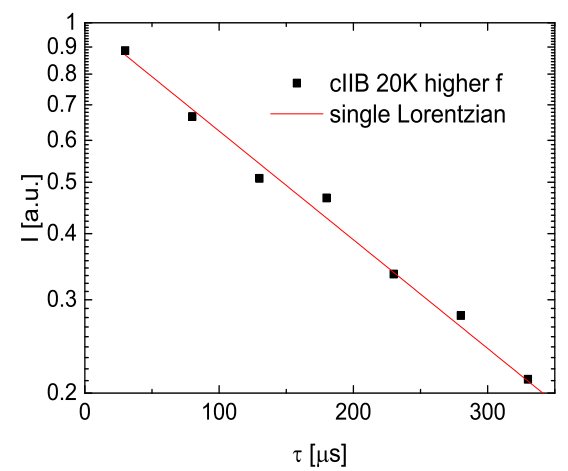

(d) Single component fit at $20 \mathrm{~K}$ for the higher frequency peak.

Figure 5.34: Double and single Lorentzian component fit of the spin-spin relaxation time for $c \| B_{0}$. 


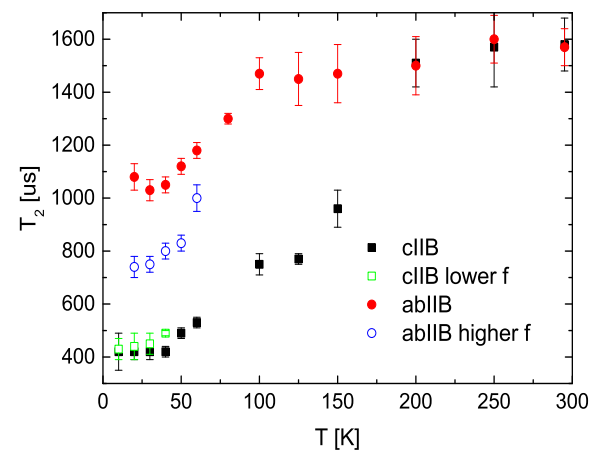

(a) $T_{2}$

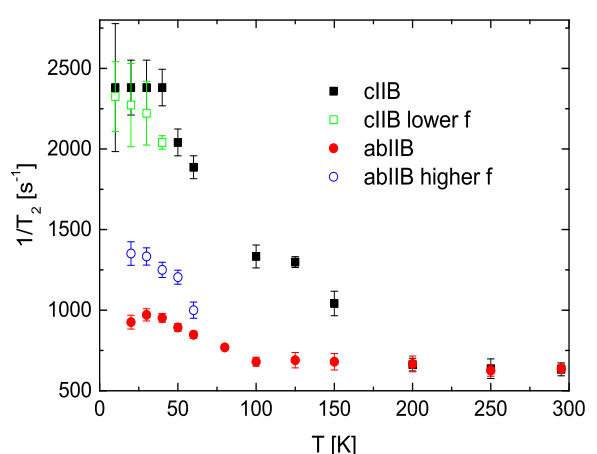

(b) $1 / T_{2}$

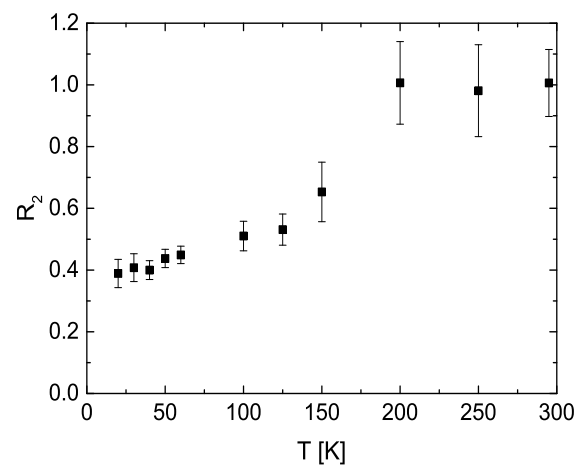

(c) $R_{2}$

Figure 5.35: Temperature dependence of the spin-spin relaxation rate and the anisotropy ratio. 


\subsection{Doping dependence of NMR properties}

The purpose of this sections is to compare the NMR results from the four different F-doped samples presented earlier and the doping dependence of other iron-based superconductors in particular other members of the 1111family.

\subsubsection{NMR spectra}

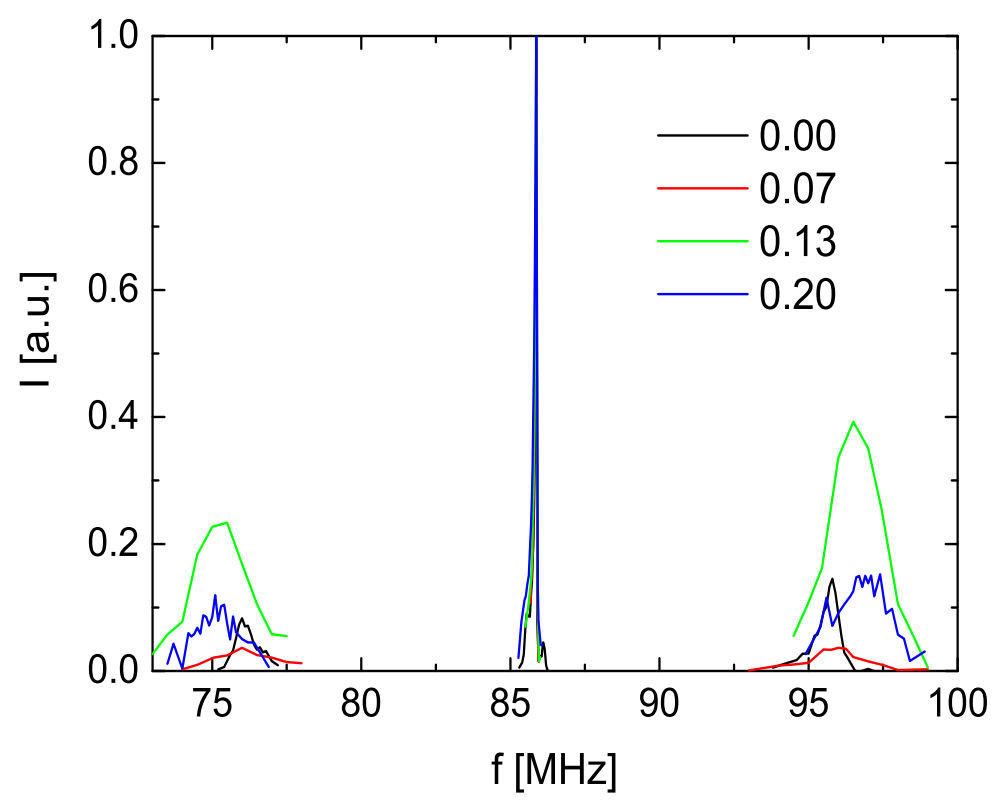

Figure 5.36: The room temperature $(295 \mathrm{~K})$ spectra for all doping in $c \| B_{0}$ including their satellite transitions.

The room temperature NMR spectra is compared in figure 5.36. There is no significant change in the resonance frequency of the CT (within errors). However, the quadrupole frequency, $f_{\mathrm{q}}$, is found to increase with increasing fluoride content. Such an increase of $f_{\mathrm{q}}$ means that the EFG is increasing in strength, which can come from an increase in charge density, as in the case of HTSC cuprates. However, the increase here is most likely caused by a change in lattice spacing instead. No change in $f_{\mathrm{q}}$ is observed in $c \| B_{0}$, but a change 
is observed in $a b \| B_{0}$. To obtain an increasing EFG strength the lattice has to contract, which is exactly what is observed in figure 5.37. A similar trend is seen throughout the 1111 family [178, 194]. The next difference is due to an increase in the fluoride content. There is a large splitting into essentially two spectra for the parent material (figure 5.4), which is only seen in $c \| B_{0}$ and is due to the SDW at around $140 \mathrm{~K}$. A similar splitting is only seen at $10 \mathrm{~K}$ in $\mathrm{x}=0.07$, also only in $c \| B_{0}$ due to a SDW at around $20 \mathrm{~K}$ (figure 5.15). There is also a very broad Gaussian peak and the NMR data indicates that there is a distribution of fluoride content. Additional peaks are observed for the superconducting samples $(x=0.13$ and $x=0.20)$, which are interpreted in terms of a variation in the fluoride concentration.

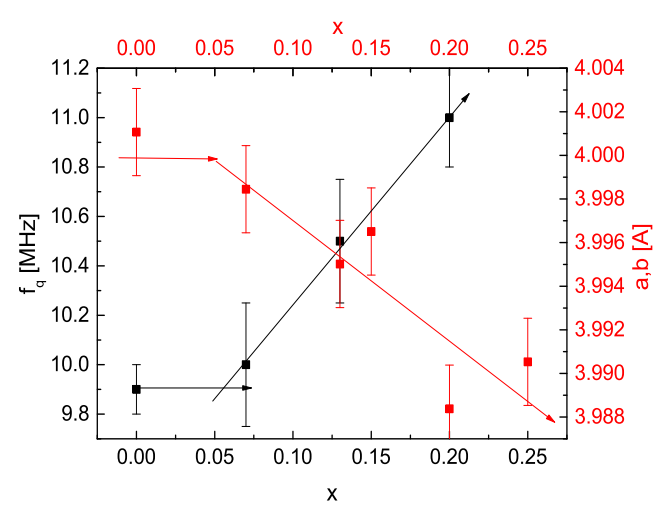

(a) Absolute changes in $f_{q}$ and $a, b$

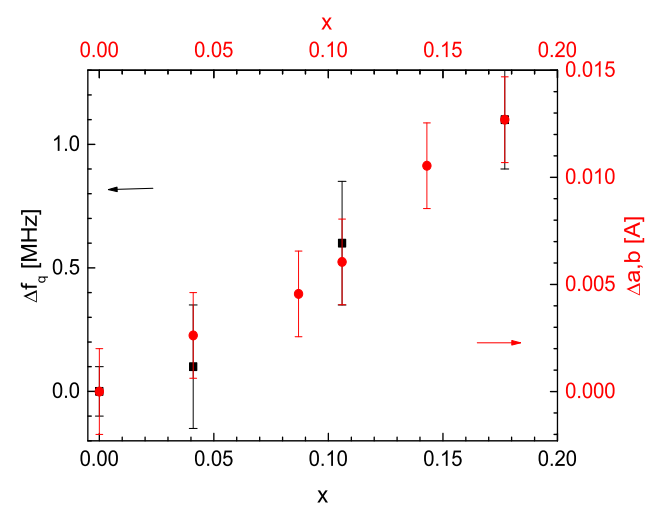

(b) Relative changes in $f_{q}$ and $a, b$

Figure 5.37: (a) Doping dependence of quadrupole frequency and lattice parameter in $c \| B_{0}$ and their relative changes in (b).

\begin{tabular}{|c|c|c|c|}
\hline Doping & $f_{\mathrm{q}}[\mathrm{MHz}]$ & lattice spacing in $a b \| B_{0}[\AA]$ & lattice spacing in $c \| B_{0}[\AA]$ \\
\hline \hline 0.00 & $9.9(1)$ & $4.001(2)$ & $8.637(4)$ \\
\hline 0.07 & $10.0(3)$ & $3.998(2)$ & $8.623(4)$ \\
\hline 0.13 & $10.5(3)$ & $3.995(2)$ & $8.612(4)$ \\
\hline 0.15 & & $3.997(2)$ & $8.613(5)$ \\
\hline 0.20 & $10.9(2)$ & $3.988(2)$ & $8.613(5)$ \\
\hline 0.25 & & $3.991(2)$ & $8.617(5)$ \\
\hline
\end{tabular}

Table 5.16: Change in quadrupole frequency and lattice spacing in $c \| B_{0}$. 


\section{NMR shift}

All temperature dependent shift data could be fitted to a $\mathrm{CW}$ temperature dependent behavior. The measured values are plotted in figure 5.38 and the obtained fitting parameters of the CW fits are shown in table 5.17. All the F-doped samples could be fitted in both magnetic field directions with a CW temperature of $\Theta=0 \mathrm{~K}$ within errors. The Knight shift is related to the spin susceptibility determined by the Ce moment. Our NMR results suggest that the real doping level for $\mathrm{x}=0.07$ is slightly below the critical point and therefore lies in a region which might have a Ce ordering. The expected ordering temperature would be close to $0 \mathrm{~K}$ and hence is within errors of our measurement. Furthermore, the Curie constant, $C$, is proportional to the hyperfine coupling constant, $A$. As mentioned in section 5.3.1 $A$ fulfills the Laplace equation with $A^{c}=-2 \cdot A^{a b}$. Hence, $C$ fulfills the Laplace equation as well which can be seen in table 5.17 for all samples within the experimental errors.

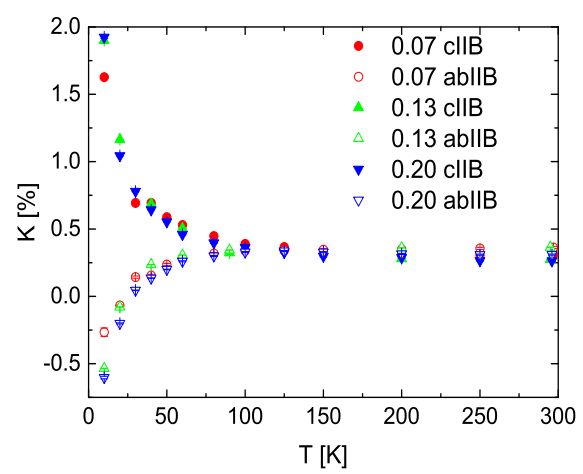

Figure 5.38: Temperature dependence of the NMR shift from the main line. 


\begin{tabular}{|c|c|c|c|}
\hline sample & $\mathrm{C}[\% \mathrm{~K}]$ & $\Theta[\mathrm{K}]$ & $\mathrm{K}_{\mathrm{L}}[\%]$ \\
\hline \hline $0.07 c \| B_{0}$ & $18(2)$ & $-1(2)$ & $0.23(1)$ \\
\hline $0.07 a b \| B_{0}$ & $-8(2)$ & $-2(2)$ & $0.40(1)$ \\
\hline $0.13 c \| B_{0}$ & $20(4)$ & $-1(2)$ & $0.17(4)$ \\
\hline $0.13 a b \| B_{0}$ & $-10(2)$ & $0(2)$ & $0.44(4)$ \\
\hline $0.20 c \| B_{0}$ & $18(1)$ & $-1(1)$ & $0.19(1)$ \\
\hline $0.20 a b \| B_{0}$ & $-11(2)$ & $-1(2)$ & $0.40(2)$ \\
\hline
\end{tabular}

Table 5.17: CW fit parameters for the temperature dependent shift of $\mathrm{CeFeAsO}_{1-\mathrm{x}} \mathrm{F}_{\mathrm{x}}$.

\section{NMR linewidth}

The NMR linewidth has a similar temperature dependence for all samples and they become broader with increasing $\mathrm{F}$ concentration as is shown in figure 5.39. All NMR data show an asymmetric lineshape at $295 \mathrm{~K}$ in $c \|$ $B_{0}$ with a lower frequency tail, due to non-perfect alignment of the micro crystals, whereas $a b \| B_{0}$ is more symmetric. The magnetic field dependence was already published in reference [193] by our group. The temperature dependence of the linewidths were fitted with a CW temperature dependence (shown in previous sections) for all samples and the results are shown in table 5.18 for the main peaks. All CW fits could be done with a CW temperature of zero within experimental uncertainty, which suggest that the NMR linewidth of the main peaks is dominated by magnetic interactions with Ce moments.

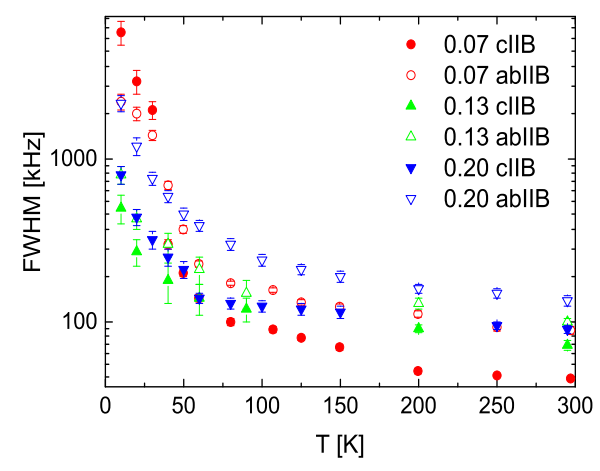

Figure 5.39: Temperature dependence of the linewidth. 


\begin{tabular}{|c|c|c|c|}
\hline sample & $\Theta[\mathrm{K}]$ & $\mathrm{b}[\mathrm{kHz}]$ & $\mathrm{a}[\mathrm{kHzK}]$ \\
\hline \hline $0.07 \mathrm{c} \| \mathrm{B}$ & $0(2)$ & $7000(500)$ & $20(3)$ \\
\hline $0.07 \mathrm{ab} \| \mathrm{B}$ & $0(2)$ & $9700(600)$ & $58(5)$ \\
\hline $0.13 \mathrm{c} \| \mathrm{B}$ & $0(2)$ & $4600(600)$ & $63(4)$ \\
\hline $0.13 \mathrm{ab} \| \mathrm{B}$ & $0(2)$ & $7500(600)$ & $78(5)$ \\
\hline $0.20 \mathrm{c} \| \mathrm{B}$ & $0(2)$ & $7000(800)$ & $60(10)$ \\
\hline $0.20 \mathrm{ab} \| \mathrm{B}$ & $0(2)$ & $21000(1000)$ & $50(10)$ \\
\hline
\end{tabular}

Table 5.18: CW fit parameters for the linewidth.

\subsubsection{Spin-lattice relaxation}

Results from $T_{1}$ measurements are plotted for all samples in figure 5.40. The major point to note here is that the relaxation rates for both superconducting samples are lower than the non-superconducting samples independent of the orientation. This difference can be explained by the existence of an additional relaxation mechanism through hyperfine interactions with Fe moments, which exhibits magnetic order only for non-superconducting samples at low temperature. The temperature dependence can be described by Moriya's theory of weakly itinerant 2D AFM for all doping levels. The obtained fitting parameters are summarized in table 5.19. No fitting attempt was made for the undoped compound, because the high transition temperature of the SDW around $140 \mathrm{~K}$ makes this impossible. 


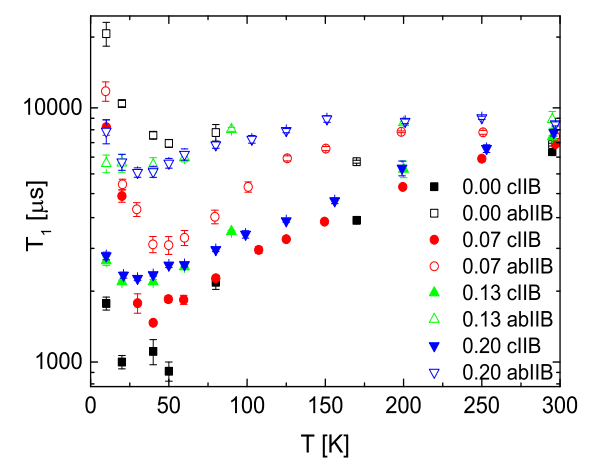

(a) Temperature dependence of $T_{1}$.

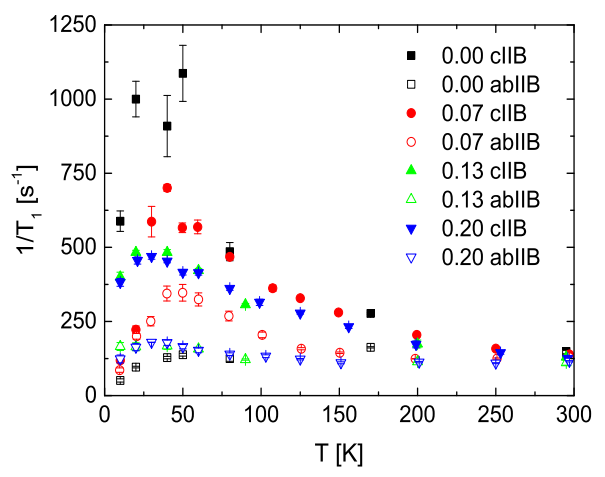

(b) Temperature dependence of $1 / T_{1}$.

Figure 5.40: Temperature dependence of the spin-lattice relaxation for all samples.

\begin{tabular}{|c|c|c|}
\hline Sample & $\mathrm{C}\left[\mathrm{s}^{-1}\right]$ & $T_{0}[\mathrm{~K}]$ \\
\hline \hline $0.07 \mathrm{c} \| \mathrm{B}$ & $270(20)$ & $450(30)$ \\
\hline $0.07 \mathrm{ab} \| \mathrm{B}$ & $35(10)$ & $9000(3000)$ \\
\hline $0.13 \mathrm{c} \| \mathrm{B}$ & $180(20)$ & $580(60)$ \\
\hline $0.13 \mathrm{ab} \| \mathrm{B}$ & $22(13)$ & $36000(11000)$ \\
\hline $0.20 \mathrm{c} \| \mathrm{B}$ & $163(7)$ & $650(60)$ \\
\hline $0.20 \mathrm{ab} \| \mathrm{B}$ & $33(4)$ & $6100(3000)$ \\
\hline
\end{tabular}

Table 5.19: Fitting parameters for 2D spin fluctuations (section 5.3.3) to figure 5.40 .

The fits for $c \| B_{0}$ are good over the whole fitting region, so it seems appropriate to say that $2 \mathrm{D}$ spin fluctuations are the major relaxation mechanism. The 2D spin fluctuation behavior is supported by the anisotropy ratio, which is shown in figure 5.41. The anisotropy ratio is roughly 1.0 at room temperature indicating a similar relaxation rate in $c \| B_{0}$ and $a b \| B_{0}$. With decreasing temperature both rates increase (figure 5.40b) for all samples, but this increase is faster in $c \| B_{0}$. The first deviation from a joint temperature-dependence for all samples occurs at the SDW transition temperature $(140 \mathrm{~K})$ for the undoped compound $\mathrm{x}=0.00$. From this temperature downwards the spin-lattice relaxation rate is enhanced through hyperfine 
coupling to the AFM ordered Fe moments and their spin fluctuations, which is an effect limited to the $c \| B_{0}$ orientation. The second deviation occurs around $100 \mathrm{~K}$ between the superconducting samples of $\mathrm{x}=0.13$ and 0.20 and the non-superconducting sample of $\mathrm{x}=0.07$ where the later sample stays at a ratio of 0.6 and the ratio for the superconducting samples reduces further before becoming stable at a ratio of around 0.4. This change occurs because of a structural transition from the tetrahedral phase to an orthorhombic phase around $100 \mathrm{~K}$ [2].

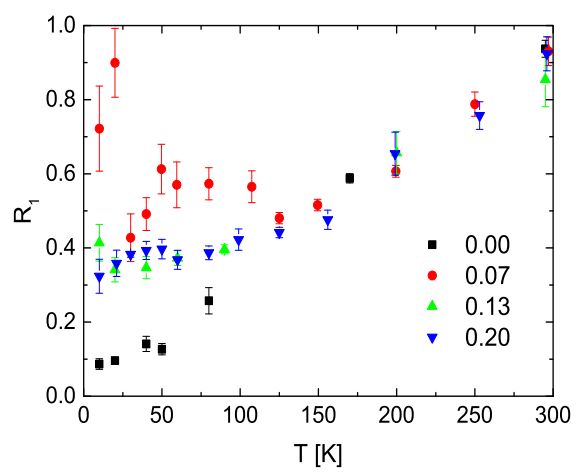

Figure 5.41: Temperature dependence of the anisotropy ratio $R_{1}$ for all doping.

\subsubsection{Spin-spin relaxation}

The temperature dependence of the spin-spin relaxation rate is weakly temperature dependent for $a b \| B_{0}$ as shown in figure 5.42a. However, for $c \| B_{0}$ there is a continuous increase with decreasing temperature. Furthermore, the relaxation rate is the highest for the undoped compound at lower temperatures, lower for the $\mathrm{x}=0.07$ sample and the lowest values are found in the superconducting samples where the $1 / T_{2}$ values are similar over the whole temperature range. The anisotropy ratio is shown in figure $5.42 \mathrm{~b}$. The temperature dependence of the spin-spin relaxation is consistent with a mechanism, which is dominated by magnetic dipole interaction with the Ce moments. An additional contribution arises in samples, which have ordered Fe moments. This can be seen in the undoped sample in figure $5.42 \mathrm{~b}$, where 
all points measured below the SDW transition show a departure from the common curve.

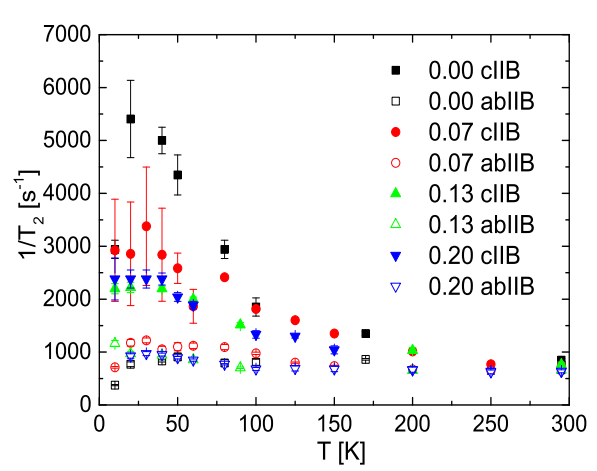

(a) $1 / T_{2}$

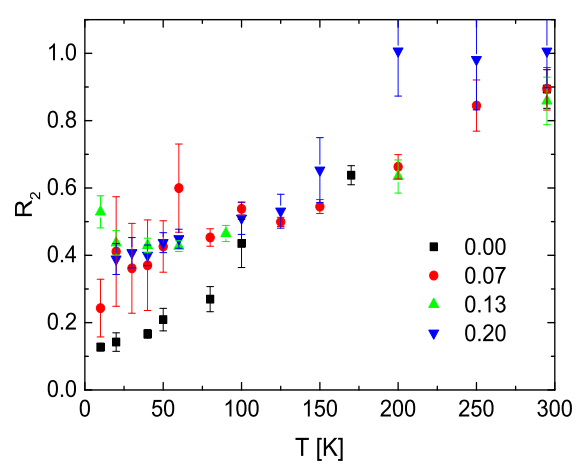

(b) $R_{2}$

Figure 5.42: Temperature dependence of the spin-spin-relaxation rate and the anisotropy ratio $R_{2}$. 


\subsection{Summary and conclusions}

In conclusion ${ }^{75} \mathrm{As}$ NMR measurements on $\mathrm{CeFeAsO}_{1-\mathrm{x}} \mathrm{F}_{\mathrm{x}}$ show the appearance of additional peaks in the low temperature region. This is due to spin-density-wave (SDW) ordering in the non-superconducting samples with $x=0.00$ and $x=0.07$. For the $x=0.07$ sample a SDW ordering temperature at around $20 \mathrm{~K}$ was inferred from the NMR data, which was not expected because a SDW should only be observed for $x \leq 0.06$. This together with the appearance of a broad low temperature NMR peak is consistent with charge inhomogeneities due to a variable fluoride concentration. Additional NMR peaks are also seen for the superconducting samples in both directions and for temperatures as high as $100 \mathrm{~K}$, which are due to charge inhomogeneities. Therefore, NMR results show that there are charge inhomogeneities for $x=0.06$ to at least $x=0.20$. Charge inhomogeneities have also been reported in the cuprate superconductors.

The NMR shifts of the main line for $x \geq 0.07$ are dominated by hyperfine coupling to the Ce moments and where the main line CW temperatures are zero within experimental errors. The additional peak at low temperatures seen in the $x=0.13$ and $x=0.20$ NMR spectra has a negative CW temperature that indicates antiferromagnetic interactions between the Ce moments. The NMR linewidths are all dominated by dipole interactions with the $\mathrm{Ce}$ moments and they display a CW temperature equal to zero within experimental errors for the main peak.

The spin-lattice relaxation can be interpreted with Moriya's theory for weakly itinerant 2D antiferromagnets [49, 190]. This mechanism is strongest in $c \| B_{0}$ because it is a hyperfine interaction, which only has non-zero matrix elements in this direction. Therefore, the spin relaxation is via $2 \mathrm{D}$ spin fluctuations. The spin-spin relaxation can be fitted with a Lorentzian shape over the whole doping range and at all temperatures. However, for $\mathrm{CeFeAsO}_{0.93} \mathrm{~F}_{0.07}$ is it necessary to use two components to obtain a satisfying fit in the low temperature region. The additional second component is very short in comparison and is due to arising spin fluctuations from Fe. 


\section{Chapter 6}

\section{Magnetotransport study of $\mathrm{AFe}_{2} \mathrm{As}_{2}(\mathrm{~A}=\mathrm{Ba}, \mathrm{Sr})$}

\subsection{Introduction}

The second group of iron-based superconductors are the ternary iron arsenide $\mathrm{AFe}_{2} \mathrm{As}_{2}(\mathrm{~A}=\mathrm{Ba}, \mathrm{Sr}, \mathrm{Ca}, \mathrm{Eu}, \mathrm{K})^{1}$ with the tetragonal $\mathrm{ThCr}_{2} \mathrm{Si}_{2}$-type structure (space group I4/mmm) [195]. They contain practically identical layers of edge-sharing FeAs tetrahedrons separated by 'A' atoms instead of the R-O layers $(\mathrm{R}=$ rare-earth) in the 1111-type. They can possess interesting properties such as a large magnetoresistance (MR) [196, 197], a Dirac-cone-like energy dispersion [198, 199] and a sizable thermoelectric effect [200, 201]. Of particular interest is the Dirac-cone-like energy dispersion (figure 6.1), because such an energy dispersion can have Dirac fermions with very high mobilities and a nearly zero effective mass, $m^{*}$ [8, 201].

\footnotetext{
${ }^{1}$ under investigation here is $\mathrm{M}=\mathrm{Ba}, \mathrm{Sr}$
} 


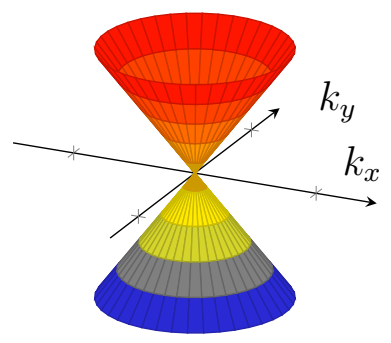

Figure 6.1: Dirac cone energy dispersion.

Under such conditions quantum linear magnetoresistance (MR) is predicted at high fields when only one Landau level is occupied and the Fermi level is close enough to the cone apex. According to Abrikosov [202] the resistivity can be written as:

$$
\rho_{x x}=\rho_{y y}=\left[\frac{N_{i}}{\pi n_{D}^{2} e}\right] \cdot B
$$

for a concentration of static scattering centres, $N_{i}$, density of carriers, $n_{D}$, and the applied magnetic field, $B$. Quantum linear MR was reported several times from magnetotransport measurements on 1111 and 122 iron-based superconductors [8, 203, 204], but arguments were made that the required fields are too high for the observation of quantum linear $\mathrm{MR}$ in $\mathrm{BaFe}_{2} \mathrm{As}_{2}$ [205] and that none was observed in good quality and homogeneous samples [196]. Therefore, $\mathrm{BaFe}_{2} \mathrm{As}_{2}$ and $\mathrm{SrFe}_{2} \mathrm{As}_{2}$ were investigated with the aim to find evidence of the existence of quantum linear MR in these crystals. 


\subsection{Synthesis and structure}

Single crystals of $\mathrm{BaFe}_{2} \mathrm{As}_{2}$ were grown by the FeAs self-flux method [206, 207], while $\mathrm{SrFe}_{2} \mathrm{As}_{2}$ single crystals were grown by the self-flux method and inductive melting of pre-synthesised polycrystalline $\mathrm{SrFe}_{2} \mathrm{As}_{2}$ [208]. For the latter, pieces of polycrystalline $\mathrm{SrFe}_{2} \mathrm{As}_{2}$ were placed inside a capped alumina "test tube" and loaded into an inductive furnace partially filled with highpurity argon gas. The alumina "test tube" was heated above $1500 \mathrm{~K}$ and then cooled down at a rate of $1 \mathrm{~K} \mathrm{~min}^{-1}$ to $1153 \mathrm{~K}$ before a rapid cool-down to room temperature. The final crystals were obtained in both cases by cleaving the bulk material. Sample quality was checked with powder X-ray diffraction, which shows sharp $c$-axis (001) Bragg reflections (figure A.3). Post-annealing was only necessary for $\mathrm{SrFe}_{2} \mathrm{As}_{2}$ crystals to remove crystallographic strain. This was done by sealing the crystals in a quartz tube partially filled with high-purity argon gas and heating to $773 \mathrm{~K}$ for two hours.

$\mathrm{Ca}$ ion were implanted using the facility at GNS Science with $20 \mathrm{keV}$ beam energy. Both surfaces of roughly $50 \mu \mathrm{m}$ thick crystals were implanted with $\mathrm{Ca}$, along the c-axis with the same fluence. The implantation depth was estimated from Monte Carlo simulation [209, 210] to be around $10 \mathrm{~nm}$ to $15 \mathrm{~nm}$ for $\mathrm{Ba}$ [211] and $16 \mathrm{~nm}$ to $22 \mathrm{~nm}$ for $\mathrm{Sr}$ [208]. Heating effects during implantation will cause serious damage in the samples hence only low values of ion current were used (typical around $1 \mu \mathrm{A}$ ). The fluence was in the order of $1 \times 10^{15}$ ions $/ \mathrm{cm}^{2}$. 


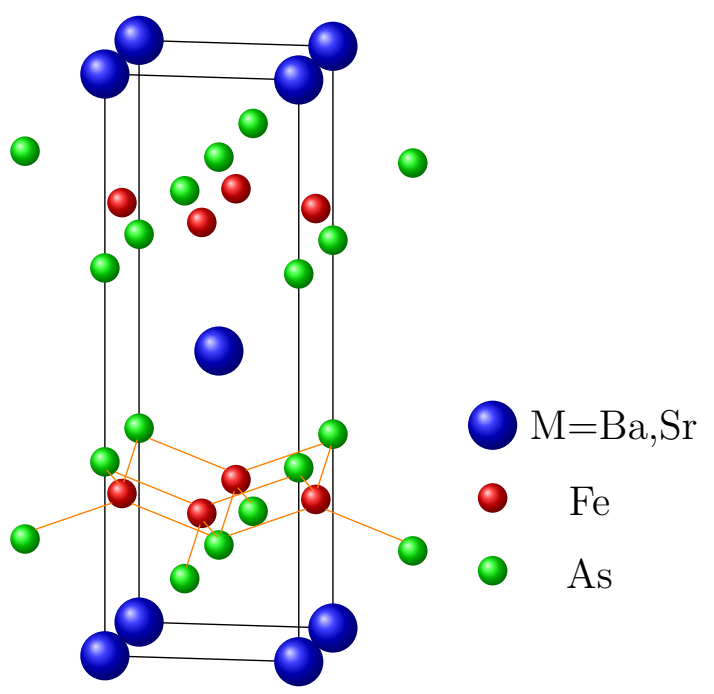

Figure 6.2: Crystal structure of $\mathrm{AFe}_{2} \mathrm{As}_{2}(\mathrm{~A}=\mathrm{Ba}, \mathrm{Sr})$. 


\subsection{Resistivity}

The resistivity of single crystalline $\mathrm{BaFe}_{2} \mathrm{As}_{2}, \mathrm{SrFe}_{2} \mathrm{As}_{2}$ and $\mathrm{SrFe}_{2} \mathrm{As}_{2}$ with 0.8 at\% Ca implanted was measured with a 4-point method and the results are shown in figure 6.3 .

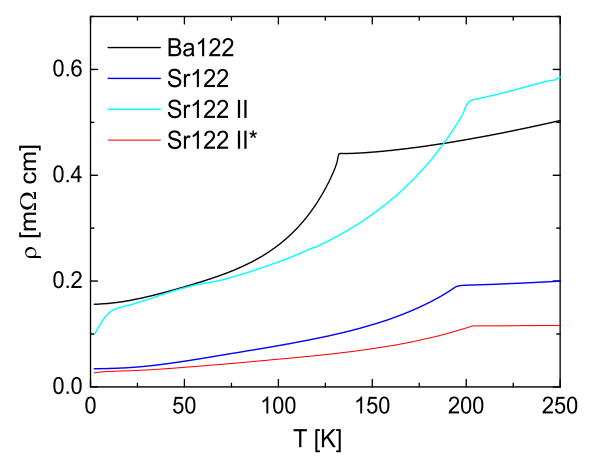

(a) Temperature dependent resistivity of $\mathrm{BaFe}_{2} \mathrm{As}_{2}$ (Ba122), $\mathrm{SrFe}_{2} \mathrm{As}_{2}(\mathrm{Sr} 122)$, 0.8 at\% Ca implanted $\mathrm{SrFe}_{2} \mathrm{As}_{2}$ (Sr122 II) and the same sample after re-annealing in $\mathrm{Ar}$ (Sr122 II*).

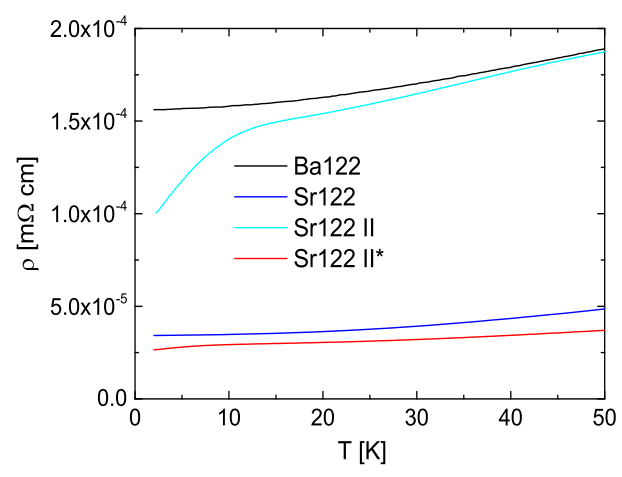

(b) Low temperature region of the temperature dependent resistivity.

Figure 6.3: Temperature-dependent resistivity of $\mathrm{BaFe}_{2} \mathrm{As}_{2}$ (Ba122), $\mathrm{SrFe}_{2} \mathrm{As}_{2}$ (Sr122), 0.8 at\%Ca implanted $\mathrm{SrFe}_{2} \mathrm{As}_{2}$ (Sr122 II) and the sample after re-annealing in $\mathrm{Ar}\left(\mathrm{Sr} 122 \mathrm{II}^{*}\right)$.

The spin-density-wave (SDW) transition causes a downturn in the resistivity in the high temperature region for all samples similar to that of $\mathrm{CeFeAsO}_{1-\mathrm{x}} \mathrm{F}_{\mathrm{x}}$ as presented in section 4.3.1. This transition occurs at $205 \mathrm{~K}$ for $\mathrm{SrFe}_{2} \mathrm{As}_{2}$ and at $135 \mathrm{~K}$ for $\mathrm{BaFe}_{2} \mathrm{As}_{2}$. No sign of superconductivity is observed in the low temperature region for the non-implanted single crystals and they follow a Fermi-liquid $T^{2}$ temperature dependent behavior down to the lowest measured temperatures. However, the Ca implanted $\mathrm{SrFe}_{2} \mathrm{As}_{2}$ single crystal shows a downturn in resistivity below $15 \mathrm{~K}^{2}$. Such a downturn in the resistivity was previously observed by our group in a more Ca-implanted $\mathrm{SrFe}_{2} \mathrm{As}_{2}$ [208] sample, which displayed a downturn at a higher temperature

\footnotetext{
${ }^{2}$ measured a month after synthesizes
} 
of $23.6 \mathrm{~K}$ and a zero resistivity state was reached at $16.4 \mathrm{~K}$. However, in this case a zero resistivity state is not reached even down to $2.2 \mathrm{~K}$, which suggests that only fractions of the implanted region are superconducting. The superconductivity results from ion implantation causing strain in the near surface region [208], similar to strain induced superconductivity in as-prepared $\mathrm{SrFe}_{2} \mathrm{As}_{2}$ samples [11, 212]. Furthermore, $T_{\mathrm{C}}$ here is similar to samples under hydrostatic pressure [213 216]. The re-annealed Ca implanted $\mathrm{SrFe}_{2} \mathrm{As}_{2}$ sample (Sr122 II*) was measured within the first week after re-annealing and hence shows no team of the strain induced superconductivity, yet. 


\subsection{Magnetoresistance}

The magnetoresistance (MR) was defined in an earlier section 3.3.1 in equation 2.11. The field dependence of the magnetoresistance was investigated up to a field of $8 \mathrm{~T}$ and their temperature dependence from room temperature down to $8 \mathrm{~K}$.

\subsubsection{Magnetoresistance of $\mathrm{BaFe}_{2} \mathrm{As}_{2}$}

Figure 6.4 shows the $\mathrm{MR}$ of $\mathrm{BaFe}_{2} \mathrm{As}_{2}$ for several temperatures between $150 \mathrm{~K}$ to $8 \mathrm{~K}$.

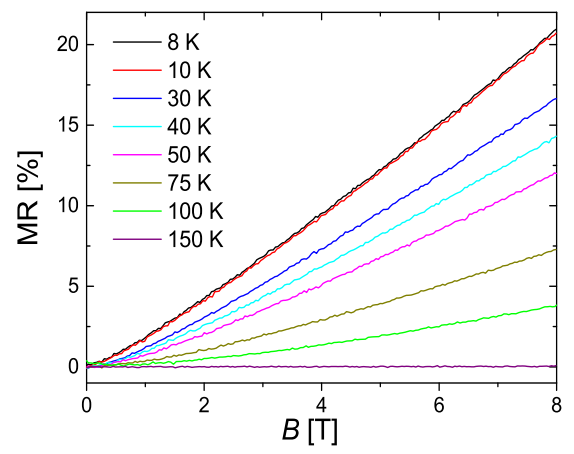

Figure 6.4: Magnetoresistance of $\mathrm{BaFe}_{2} \mathrm{As}_{2}$.

There is no noticeable MR above the SDW transition temperature at $135 \mathrm{~K}$. However, there is a rapid increase in the MR for temperatures below the SDW transition. This is due to the antiferromagnetic ordering from SDW and contributions from multiband effects plus the possibility of quantum linear MR [8, 202, 217, 218]. A maximum MR of $22 \%$ was observed at a magnetic field of $8 \mathrm{~T}$ and at a temperature of $8 \mathrm{~K}$. In a previous study, Ishida et al [196] reported a maximum MR of $280 \%$ at a magnetic field of $7 \mathrm{~T}$ and a temperature of $5 \mathrm{~K}$, also in single crystalline $\mathrm{BaFe}_{2} \mathrm{As}_{2}$ samples, which is significantly higher than the values observed here.

To examine the linearity of the MR data, the MR for all temperatures in the high field region (above $2 \mathrm{~T}$ ) was fitted to a power-law behavior with: 


$$
M R(B)=a_{1} B^{m}
$$

A systematic reduction in the exponent $m$ with decreasing temperature is found as shown in table 6.1.

\begin{tabular}{|c|c|}
\hline Temperature $[\mathrm{K}]$ & $m$ \\
\hline \hline 8 & $1.15(1)$ \\
\hline 10 & $1.16(1)$ \\
\hline 30 & $1.22(1)$ \\
\hline 40 & $1.26(2)$ \\
\hline 50 & $1.35(2)$ \\
\hline 75 & $1.35(1)$ \\
\hline 100 & $1.48(1)$ \\
\hline
\end{tabular}

Table 6.1: Temperature dependence of the exponent $m$ from a power-law fit (equation 6.2 to the high-field region of the MR.

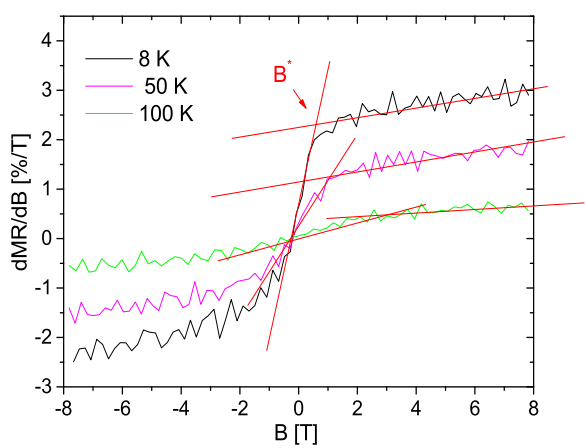

Figure 6.5: Derivative of the MR in the low-field region.

A completely different magnetic field dependence was observed at low applied magnetic fields, which is shown in figure 6.5 through the derivative of the MR. The MR behavior is different between the low-field and the highfield region, with a more rapid increase in MR in the low-field region. The MR trends toward saturation, or displays signs of linearity, in the high field region. In a quantum linear MR model, as described by Abrikosov [202, 218], such a change can be attributed to a crossover from multi-level MR to quantum 
linear MR [8, 203, 204, 217] as the magnetic field increases. The crossover occurs at a field, $B^{\star}$, where the energy splitting, $\triangle_{\mathrm{LL}}$, between the 0 th and the first Landau level:

$$
\triangle_{\mathrm{LL}}= \pm \nu_{\mathrm{F}} \sqrt{2 e \hbar B}
$$

is equal to the Fermi energy, $E_{\mathrm{F}}$, and thermal fluctuations at finite temperatures

$$
\triangle_{\mathrm{LL}}=E_{\mathrm{F}}+k_{\mathrm{B}} T
$$

Then $B^{\star}$ can be written as:

$$
B^{\star}=\frac{1}{2 e \hbar \nu_{\mathrm{F}}^{2}}\left(k_{\mathrm{B}} T+E_{\mathrm{F}}\right)^{2}
$$

Therefore, the Fermi velocity, $\nu_{\mathrm{F}}$, and the Fermi energy, $E_{\mathrm{F}}$, can be estimated from the temperature dependence of $B^{\star}$. Figure 6.6 shows the temperature dependence of $B^{\star}$ for $\mathrm{BaFe}_{2} \mathrm{As}_{2}$, with a Fermi energy, $E_{\mathrm{F}}=7.1 \mathrm{meV}$, and Fermi velocity, $\nu_{\mathrm{F}}=2.5 \times 10^{5} \mathrm{~m} \mathrm{~s}^{-1}$. Similar values were observed in earlier measurements by Tanabe et al. [8] with $E_{\mathrm{F}}=2.5 \mathrm{meV}$ to $11 \mathrm{meV}$ and $\nu_{\mathrm{F}}=\sim 2 \times 10^{5} \mathrm{~m} \mathrm{~s}^{-1}$ for $\mathrm{BaFe}_{2-\mathrm{x}} \mathrm{Ru}_{\mathrm{x}} \mathrm{As}_{2}$ and by Huynh et al. [217] with $E_{\mathrm{F}}=1(5) \mathrm{meV}$ and $\nu_{\mathrm{F}}=1.88 \times 10^{5} \mathrm{~m} \mathrm{~s}^{-1}$ on $\mathrm{BaFe}_{2} \mathrm{As}_{2}$ with well documented Dirac cone states.

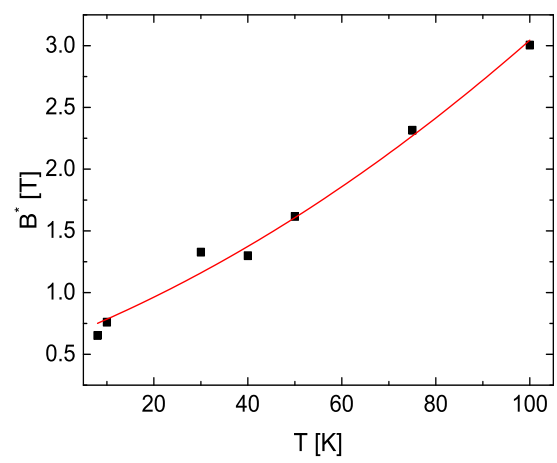

Figure 6.6: Temperature dependence of $B^{\star}$.

An absence of a linear MR at high fields was noted by Terashima et al. 
[205] and Tanabe et al. [8], where they argued that the field required to fill only one Landau level is far above our maximum field of $8 \mathrm{~T}$. Hence, the $\mathrm{MR}$ in the high field region is not dominated by quantum linear MR and can be better described using a conventional three-band transport model [197], similar to a previous study from my group on a $\mathrm{SrFe}_{2} \mathrm{As}_{2}$ sample that showed superconducting and normal-state regions [208]. This is also consistent with a study on $\mathrm{BaFe}_{2} \mathrm{As}_{2}$ crystals that were annealed to remove defects where no quantum linear MR was observed and it could be described by a three-carrier model over the measured magnetic field range [196].

\subsubsection{Magnetoresistance of $\mathrm{SrFe}_{2} \mathrm{As}_{2}$}

The MR for the non-superconducting $\mathrm{SrFe}_{2} \mathrm{As}_{2}$ crysta $]^{3}$ is slightly higher than that of the $\mathrm{BaFe}_{2} \mathrm{As}_{2}$ crystal described in the previous section. Figure 6.7 shows the MR for $\mathrm{SrFe}_{2} \mathrm{As}_{2}$ with a maximum MR of $31 \%$ for a magnetic field of $8 \mathrm{~T}$ and temperature from 2 to $10 \mathrm{~K}$.

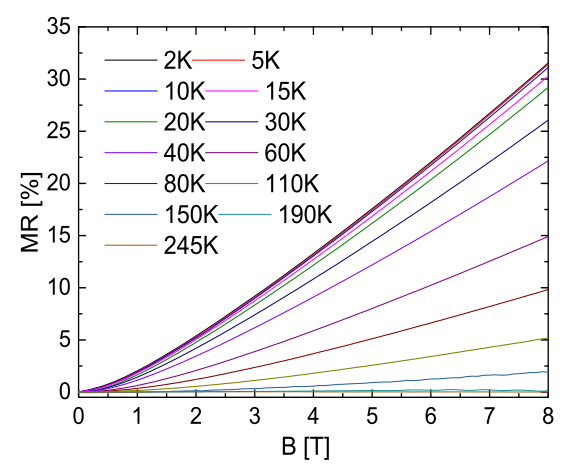

Figure 6.7: The magnetoresistance of the non-superconducting $\mathrm{SrFe}_{2} \mathrm{As}_{2}$ crystal at different temperatures.

The high field region above $2 \mathrm{~T}$ can be fitted to a power-law (equation 6.2) similar to $\mathrm{BaFe}_{2} \mathrm{As}$ with a systematic decrease in the exponent $\mathrm{m}$ shown in table 6.2, which becomes more linear (closer to 1.0) with decreasing temperature and high applied magnetic field. A crossover in the derivative of

\footnotetext{
${ }^{3}$ less then a month after synthesis
} 
the MR as shown in figure 6.8 is also found.

\begin{tabular}{|c|c|}
\hline Temperature $[\mathrm{K}]$ & $m$ \\
\hline \hline 2 & $1.26(1)$ \\
\hline 5 & $1.26(1)$ \\
\hline 10 & $1.26(1)$ \\
\hline 15 & $1.26(1)$ \\
\hline 20 & $1.27(1)$ \\
\hline 30 & $1.28(1)$ \\
\hline 40 & $1.30(1)$ \\
\hline 60 & $1.36(2)$ \\
\hline 80 & $1.44(3)$ \\
\hline 110 & $1.55(3)$ \\
\hline 150 & $1.75(4)$ \\
\hline
\end{tabular}

Table 6.2: Temperature dependence of the exponent $m$ from a power-law fit (equation 6.2) to the high-field region of the magnetoresistance.

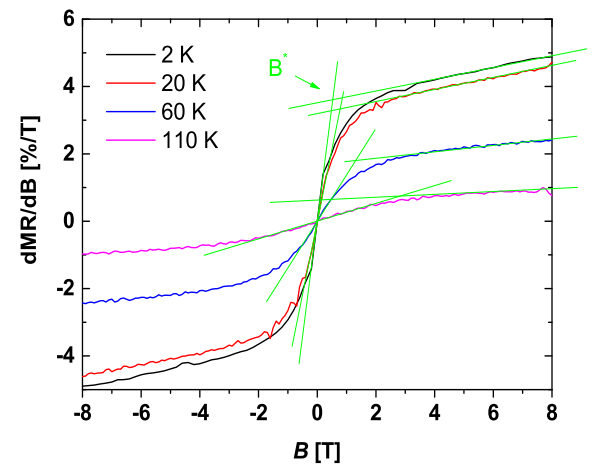

Figure 6.8: Derivative of the magnetoresistance for $\mathrm{SrFe}_{2} \mathrm{As}_{2}$ at different temperatures.

Equation 6.5 can be used to analyze $B^{\star}$ although the fit is worse than that for $\mathrm{BaFe}_{2} \mathrm{As}_{2}$. Here, the Fermi energy is $10(2) \mathrm{meV}$ and the Fermi velocity is $3.0(4) \times 10^{5} \mathrm{~m} \mathrm{~s}^{-1}$. These values suggest that the Fermi level would be too high to be able to observe a quantum linear MR below a magnetic field of $8 \mathrm{~T}$. Therefore, a three carrier-model is probably a better description than a high-field quantum linear MR. 


\subsubsection{Magnetoresistance of $\mathrm{SrFe}_{2} \mathrm{As}_{2}$ with 0.8 at\% $\mathrm{Ca}$ implantation}

The MR is shown in figure 6.9, which is very similar to that of $\mathrm{SrFe}_{2} \mathrm{As}_{2}$ from the previous section for temperatures of $20 \mathrm{~K}$ and above where a maximum of $24 \%$ is reached. The power-law fit to the high-field region shows a systematic decrease as shown in table 6.3. However, the MR below $20 \mathrm{~K}$ is different from those at higher temperatures and for the non-implanted sample. The major difference is a large initial rise in the MR, which can be clearly seen at $2 \mathrm{~K}$. The maximum $\mathrm{MR}$ at $2 \mathrm{~K}$ and $8 \mathrm{~T}$ is as high as $47 \%$, which is significantly larger than that of the non-implanted sample (31\%).

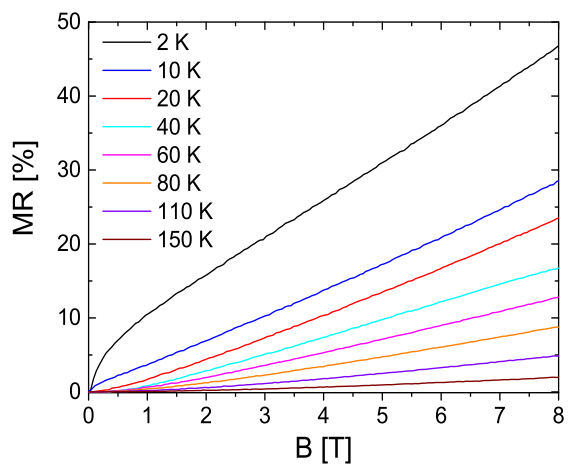

Figure 6.9: $\mathrm{MR}$ for the non-superconducting $\mathrm{SrFe}_{2} \mathrm{As}_{2}$ crystal with 0.8 at\% Ca ions implanted at different temperatures.

\footnotetext{
${ }^{4}$ for $20 \mathrm{~K}$ and $8 \mathrm{~T}$
} 


\begin{tabular}{|c|c|}
\hline Temperature $[\mathrm{K}]$ & $m$ \\
\hline \hline 2 & $1.08(1)$ \\
\hline 10 & $1.14(1)$ \\
\hline 20 & $1.15(1)$ \\
\hline 40 & $1.19(2)$ \\
\hline 60 & $1.17(3)$ \\
\hline 80 & $1.23(2)$ \\
\hline 110 & $1.33(3)$ \\
\hline 150 & $1.39(3)$ \\
\hline
\end{tabular}

Table 6.3: Temperature dependence of the exponent $m$ from a power-law fit (equation 6.2) to the high-field region of the MR.

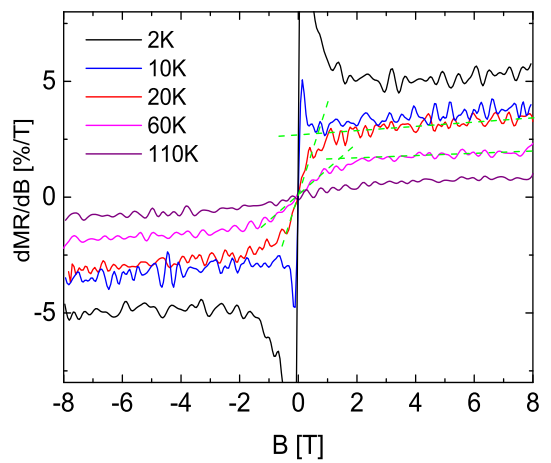

Figure 6.10: Derivative of the MR for $\mathrm{SrFe}_{2} \mathrm{As}_{2}$ with 0.8 at\% Ca implantation at different temperatures.

The magnetic field dependence of the MR is also different, which is apparent in the derivative of the MR shown in figure 6.10. The derivatives below $20 \mathrm{~K}$ are significantly larger at low fields and also reach a nearly constant value at higher fields. The different MR behavior is most likely due to parts of the near surface region becoming superconducting, which is evident in the resistivity data shown earlier in section 6.3. An increase in the magnetic field drives the weak-links in the superconducting region to normal, which leads to an initial rise of the MR. A constant MR is achieved as soon as all weak-links are driven normal by the applied field. Such MR behavior is well known in HTSC, for example in granular samples of $\mathrm{YBa}_{2} \mathrm{Cu}_{3} \mathrm{O}_{\mathrm{y}}$ high 
temperature superconductors [219]. However, the MR for the 0.8 at.\% Ca implanted $\mathrm{SrFe}_{2} \mathrm{As}_{2}$ crystal is still on increasing above $1.6 \mathrm{~T}$. This means not all weak-links have been driven normal, which is consistent with the fact that the out-of-plane upper critical field, $B_{\mathrm{c} 2}^{c}$, for this type of superconductor is higher than $8 \mathrm{~T}$ below $10 \mathrm{~K}$ [208]. It is unlikely that the remaining signal is caused by quantum linear transport. The more likely explanation is inhomogeneous current transport in the near surface region through normal and superconducting regions. Such a model was used to explain the bulk MR in a $\mathrm{SrFe}_{2} \mathrm{As}_{2}$ sample that displayed an inhomogeneous state with superconducting and normal state regions after being left in a desiccator for seven months [197. 


\subsection{The aging effect on $\mathrm{SrFe}_{2} \mathrm{As}_{2}$}

The unimplanted $\mathrm{SrFe}_{2} \mathrm{As}_{2}$ single crystal shows no sign of superconductivity after the initial annealing. These crystals were stored in a vacuum desiccator with a pressure of around 0.2 bar yet they still developed a superconducting transition at low temperatures. This process is reversible through re-annealing in a pure Ar atmosphere.

\subsubsection{Resistivity}

Resistivity measurements were carried out on the freshly made sample, after six and nine months in the desiccator and after re-annealing the 9-month-old sample, which are shown in figure 6.11.

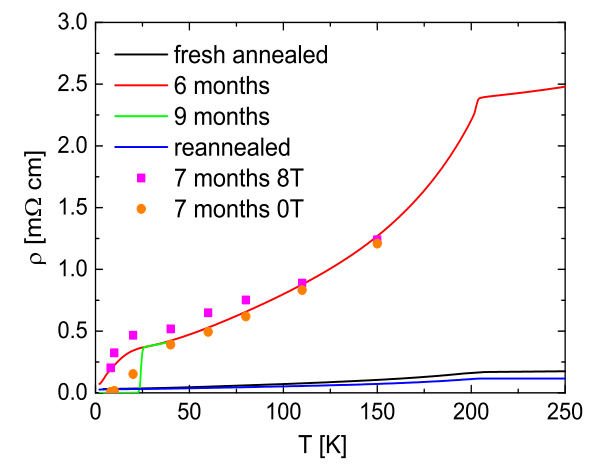

(a) Whole temperature region.

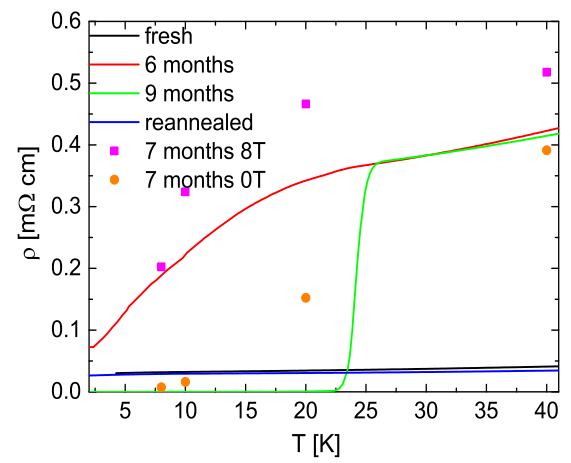

(b) Low temperature region.

Figure 6.11: Temperature dependent resistivity of the unimplanted $\mathrm{SrFe}_{2} \mathrm{As}_{2}$ single crystal freshly annealed (black), after 6 months (red), after

9 Months (green) and after re-annealing (blue). The solid symbols are values from MR measurements after 7 months at zero (orange circles) and at $8 \mathrm{~T}$ (magenta squares).

The resistivity increases when left in the desiccator and develops a low temperature downturn after six months (red curve in figure 6.11) without reaching zero resistivity. This indicates the presence a mixed electronic state with superconducting and normal regions, where the superconducting region is still below the percolation threshold. After being left for a further 
three months (green curve in figure 6.11) in the desiccator a full superconducting transition is observed at $24 \mathrm{~K}$. This value for $T_{\mathrm{C}}$ is similar to the value reported previously on ion-implanted $\mathrm{SrFe}_{2} \mathrm{As}_{2}$ [208]. Furthermore, the temperature of the SDW transition of $210 \mathrm{~K}$ stays constant, which excludes charge-doping as a likely candidate to cause the superconductivity at lower temperatures [11]. However, it is known that exposure to air [11] or water vapor [220] can induce a superconducting phase. Hence, the superconductivity in $\mathrm{SrFe}_{2} \mathrm{As}_{2}$ is most likely through air exposure, which then induces superconductivity through strain. This process is reversible through re-annealing in an Ar atmosphere, as it can be seen in figure 6.11 with the blue curve.

\subsubsection{Magnetoresistance above $T_{\mathrm{C}}$}

The MR for the freshly annealed sample is shown in section 6.4.2. The MR was measured again after seven months in the desiccator (shown in figure 6.12) and the resistivity values from the MR measurements (solid symbols) are added for comparison in figure 6.11.

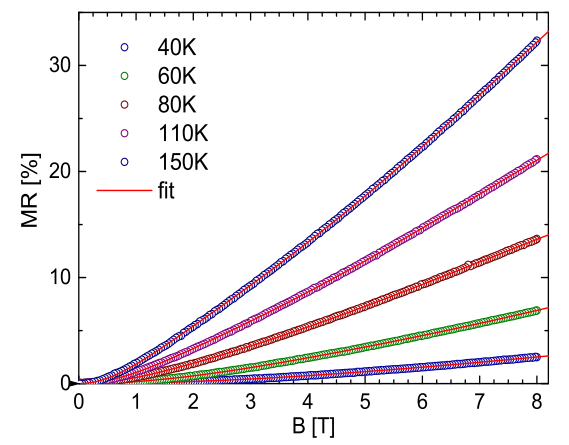

Figure 6.12: The MR of the seven months old $\mathrm{SrFe}_{2} \mathrm{As}_{2}$ crystal above the superconducting region ( $40 \mathrm{~K}$ and higher) with the data as open symbols and the solid lines being the fits to a three-carrier model.

The MR reaches a value of $30 \%$ at $40 \mathrm{~K}$ and at a field of $8 \mathrm{~T}$, which is nearly $10 \%$ higher than the freshly annealed sample at the same temperature and field. This MR was similarly analyzed in terms of a crossover to quantum 
linear MR (figure 6.13a). The temperature dependent crossover fields are shown in figure 6.13b.

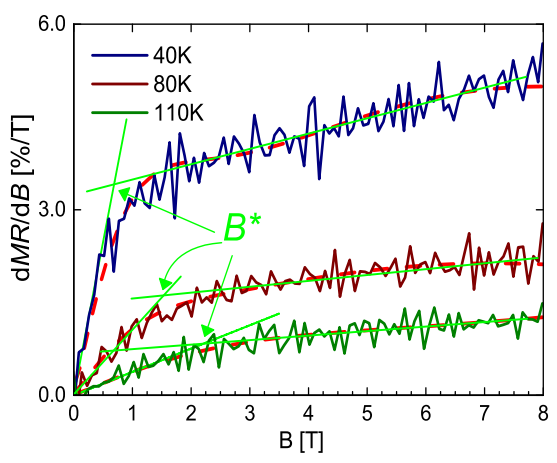

(a) The derivative of the MR for the seven months old $\mathrm{SrFe}_{2} \mathrm{As}_{2}$ crystal.

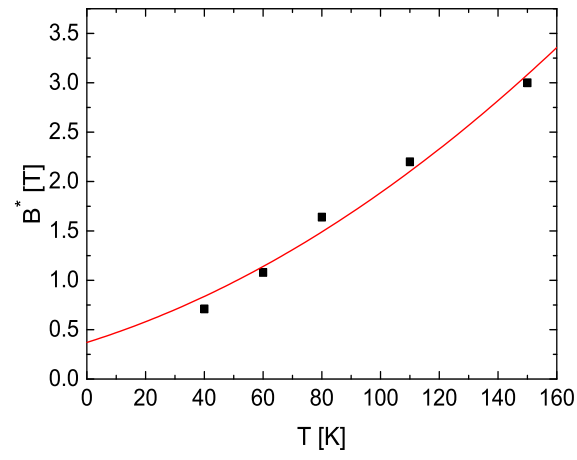

(b) The crossover field, $B^{*}$, from equation 6.5 to the MR of the seven month old $\mathrm{SrFe}_{2} \mathrm{As}_{2}$ crystal.

Figure 6.13: $B^{*}$ analysis for the seven months old $\mathrm{SrFe}_{2} \mathrm{As}_{2}$ crystal.

This $B^{*}$ can then be fitted to equation 6.5 , which is shown in figure 6.13b. A Fermi energy of $7(2) \mathrm{meV}$ and a Fermi velocity of $3.1(3) \times 10^{5} \mathrm{~m} \mathrm{~s}^{-1}$ are obtained from this fit. The Fermi energy is slightly lower than that for the fresh sample whereas no change in the Fermi velocity can be noted. Therefore, these values seem to be sensible despite the fact that the observed MR never becomes linear in the high field region and hence the MR cannot completely be attributed to a quantum linear MR.

As suggested earlier a multi-carrier model might be the more appropriate explanation. In such a case the change in MR would come from a change in the carrier mobility and concentration with temperature [196]. The data can be modeled by a three-carrier model with one hole and two electron bands, which seems reasonable considering the band structure and a report from quantum oscillation measurements on $\mathrm{BaFe}_{2} \mathrm{As}_{2}$ [205], where the appearance of hole and electron pockets at the Fermi level was reported. Kim's [111] 3-carrier matrix formalism for the longitudinal MR was used to analyze the observed MR, where the MR can be written as, 


$$
M R=\frac{\left[\left(\alpha+\gamma B^{2}\right) B^{2}\right]}{\left[1+\left(\beta+\delta B^{2}\right) B^{2}\right]}
$$

as described earlier in section 2.5.2. The total carrier density below the SDW transition was determined to be in the order of $1 \times 10^{20} / \mathrm{cm}^{3}$ in Hall measurements [125], optical conductivity measurements [15] and density functional theory (DFT) calculations [221]. They all show that $n_{i}$ is temperature dependent and the hole density should equal the sum of the electron densities, $n_{\mathrm{h}}=n_{\mathrm{e} 1}+n_{\mathrm{e} 2}$. The fit to the data is shown in figure 6.12 as solid red lines. The obtained carrier mobilities and densities are shown in figure 6.14a and $6.14 \mathrm{~b}$.

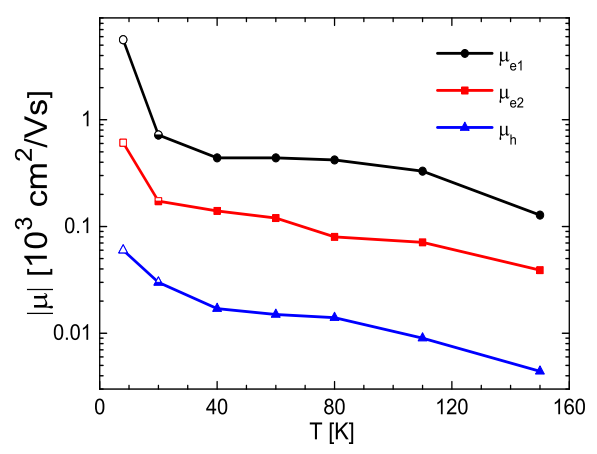

(a) Carrier mobility

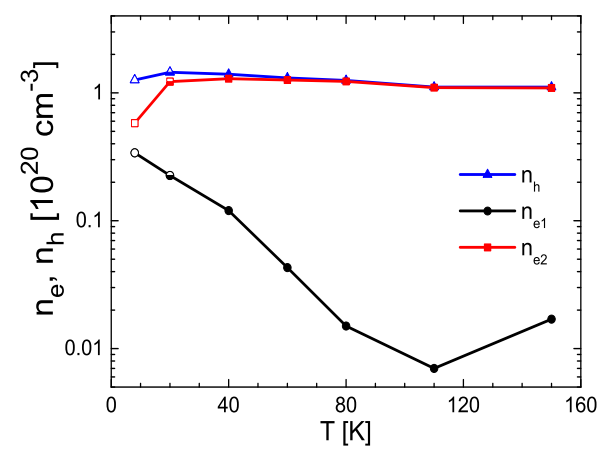

(b) Carrier densities

Figure 6.14: Temperature dependence of the (a) carrier mobilities and (b) the carrier densities obtained from the fit to Kim's three-carrier model.

Furthermore, figure 6.13a shows a fit to the three-carrier model to the derivative of the MR as solid red line. Both fits are good over the measured field range and the calculated zero-field resistivity from the fitted parameters,

$$
\rho_{x x}=1 / \sum\left|e n_{i} \mu_{i}\right|
$$

is in the same order of magnitude with $0.2 \mathrm{~m} \Omega \mathrm{cm}$ and below. Fitting of the MR by manually changing $n_{i}$ has no significant effect on the temperature dependent behavior of $\left|\mu_{i}\right|$ and $\mu_{\mathrm{e} 1}>\mu_{\mathrm{e} 2}>\mu_{\mathrm{h}}$ stays valid, despite changes in the magnitude of $\mu_{i}$. Hence, these fits show a reasonable agreement with the 
measured data, however the fixed values of $n_{i}$ and $\mu_{i}$ lead to an inconsistent value of $\rho_{x x}$ from equation 6.7, which is lower by a factor of 2 to 30 compared with the observed resistivity (figure 6.11).

\subsubsection{Magnetoresistance below $T_{\mathrm{C}}$}

The MR for temperatures below $T_{\mathrm{C}}$ are shown in figure 6.15a, together with the $\mathrm{MR}$ at $40 \mathrm{~K}$ for comparison. The $\mathrm{MR}$ is significantly enhanced below $T_{\mathrm{C}}$, with a value of $197 \%$ at $8 \mathrm{~T}$ and $20 \mathrm{~K}$. This enhancement of the MR is due to a suppression of superconductivity by the applied magnetic field in the superconducting regions together with a 3-carrier transport in the metallic and non-superconducting regions, resulting in an inhomogeneous state below $T_{\mathrm{C}}$. The low field part of the MR shows an initial increase in the MR, which starts to saturate around $1 \mathrm{~T}$. This is a sign of suppressed superconductivity, which is even clearer in the derivative of the MR shown in figure 6.15b, with a large increase below $1 \mathrm{~T}$.

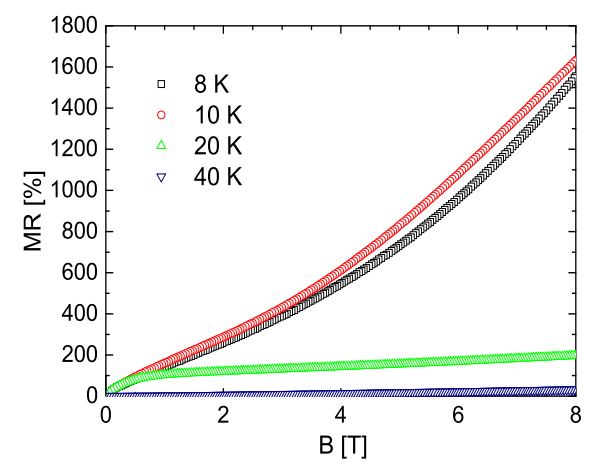

(a) Field dependence of the MR below $T_{\mathrm{C}}$ and at $40 \mathrm{~K}$ for comparison.

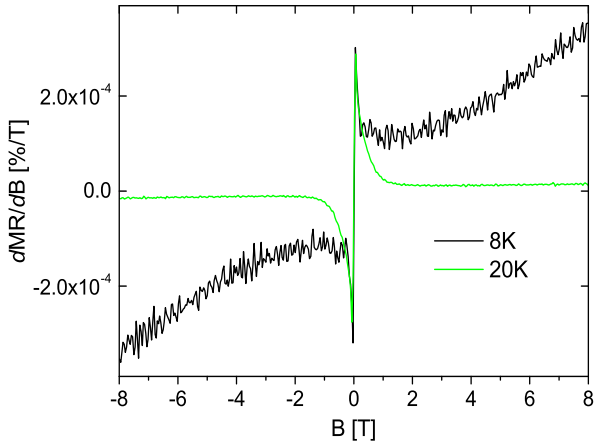

(b) Field dependence of the derivative of the MR.

Figure 6.15: The magnetoresistance below the superconducting transition.

The upper critical field, $B_{c 2}(\sim 1 \mathrm{~T}$ at $20 \mathrm{~K})$, measured on a strain-induced $\mathrm{SrFe}_{2} \mathrm{As}_{2}$ crystal [208] is roughly the same as the observed crossover field around $\sim 1 \mathrm{~T}$. Furthermore, the resistivity at $8 \mathrm{~T}$ (figure 6.11) is very close to the expected resistivity for the normal state when the MR is dominated 
by 3-carrier transport. Hence, it makes sense to assume that the MR, at a field which is sufficiently high above the crossover field, is dominated by a three-carrier transport. This can be confirmed by fitting the data to equation 6.6, which was simplified under the assumptions that there are no superconducting regions above a certain field:

$$
M R(B)=\frac{M R^{\prime}(B)}{\zeta}+\frac{1}{\zeta}-1
$$

with the three-carrier $\mathrm{MR}$ in the absence of superconductivity, $M R^{\prime}(B)$, given by equation 6.6 and $\zeta=\rho^{\prime}(0) / \rho(0)$, where $\rho^{\prime}(0)$ is defined as the resistivity at zero field and without superconductivity. A good fit is obtained with $\zeta(20 K)=0.506$. The resultant mobilities and carrier densities are shown in figure 6.14 as the half-filled symbols. These values continue the trend seen in the higher temperature data.

The MR at $8 \mathrm{~K}$ increases only up to a field of $\sim 0.2 \mathrm{~T}$ before the magnetic field dependence changes, which results in a MR of $1600 \%$ at $8 \mathrm{~T}$. The derivative shown in figure $6.15 \mathrm{~b}$ indicates that this change is even more pronounced. Josephson coupled weak-links as observed in superconductors with poorly connected superconducting regions (for example, in grain-aligned bulk $\mathrm{YBa}_{2} \mathrm{Cu}_{3} \mathrm{O}_{\mathrm{x}}$ ) [219, 222] are a possible explanation for the initial rise in the MR. In this case the change in the magnetic-field dependence of the $\mathrm{MR}$ at $\sim 0.2 \mathrm{~T}$ suggests that the weak-links are all driven normal for applied magnetic fields $B>0.2 \mathrm{~T}$ and that the current percolation pathway is poorly connected between the superconducting regions. However, the suppression of superconductivity by the applied magnetic field cannot explain the MR at larger fields, because $B_{\mathrm{c} 2}$ measured in $\mathrm{SrFe}_{2} \mathrm{As}_{2}$ crystals and films [11, 208, 220] is far larger than the maximum applied field used here $(8 \mathrm{~T})$. Furthermore, the irreversibility field at $8 \mathrm{~K}$ is close to the maximum applied field of $8 \mathrm{~T}$ in superconducting samples [208, 220] and hence flux flow is not expected below $<8 \mathrm{~T}$. Hence, vortex motion, which leads to a large MR with increasing fields in superconductors, is also unlikely to be the reason.

A possible model is suggested by the appearance of a finite resistance at $8 \mathrm{~K}$ where metallic and non-superconducting $\mathrm{SrFe}_{2} \mathrm{As}_{2}$ contains supercon- 
ducting droplets. Then the percolation pathway would include conduction in the metallic regions and effectively shorted out superconducting regions. Hence, the MR above $\sim 1 \mathrm{~T}$ might be completely due to the metallic regions given by equation 6.6. Furthermore, figure 6.15a shows the fit using equation 6.8 above $1 \mathrm{~T}$ to the data, which provides a good agreement with $\zeta(8 K)=0.807$. The mobilities (figure 6.14) are further enhanced with respect to the higher temperature values. Such a large low-temperature enhancement was not reported by Ishida et al. [196] on non-superconducting $\mathrm{BaFe}_{2} \mathrm{As}_{2}$ with a mobility of $\mu_{\mathrm{e} 1}=4.5 \times 10^{3} \mathrm{~cm}^{2} / \mathrm{Vs}$ at $2 \mathrm{~K}$, although the mobilities did increase with deceasing temperature. The $\mu_{\mathrm{e} 1}=5.6 \times 10^{3} \mathrm{~cm}^{2} / \mathrm{Vs}$ presented here at $8 \mathrm{~K}$ is only slightly larger than that from Ishida et al. [196]. For $\mathrm{SrFe}_{2} \mathrm{As}_{2}$ a possible explanation is that part of the low-temperature increase in the mobilities may be a consequence of the inhomogeneous state and the complex percolation pathway.

\subsubsection{Reversibility}

The aged $\mathrm{SrFe}_{2} \mathrm{As}_{2}$ crystal, which became superconducting after nine months can be reversed to its initial state by re-annealing the crystals in a pure $\mathrm{Ar}$ atmosphere similar to the last annealing step done on the new samples. This suggests that superconductivity in this sample is caused by air exposure, which causes strain in the sample subsequently inducing superconductivity [11. The resistivity of the re-annealed sample is shown in figure 6.11 displaying good agreement with the initial measurement on a fresh sample. The MR is shown in figure 6.16 with the same field and temperature dependence as those shown in figure 6.7. 


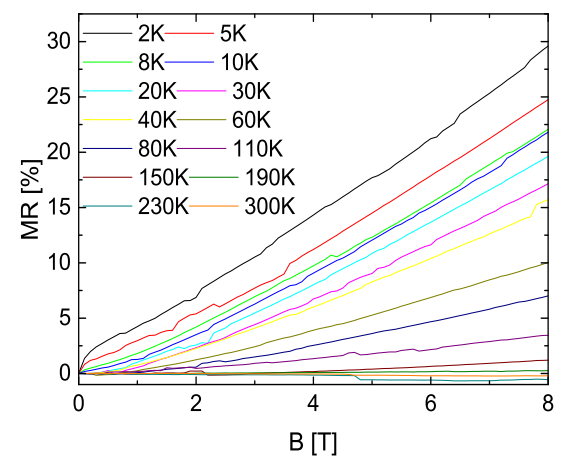

Figure 6.16: Magnetic field dependence of the MR for different temperatures for the re-annealed $\mathrm{SrFe}_{2} \mathrm{As}_{2}$ crystal.

\subsection{Summary and conclusions}

The magnetoresistance of $\mathrm{BaFe}_{2} \mathrm{As}_{2}, \mathrm{SrFe}_{2} \mathrm{As}_{2}$ and low energy Ca implanted $\mathrm{SrFe}_{2} \mathrm{As}_{2}$ crystals were studied. $\mathrm{BaFe}_{2} \mathrm{As}_{2}$ shows no sign of superconductivity, while $\mathrm{SrFe}_{2} \mathrm{As}_{2}$ is non-superconducting in the initial state and develops superconductivity over time. $\mathrm{SrFe}_{2} \mathrm{As}_{2}$ with 0.8 at\% Ca shows a low temperature downturn indicating a developing superconducting state. The MR is positive for all three samples and the derivative of the MR displays a crossover between a low field and a high field region. It has been suggested that the MR in the high field region can be described by quantum linear MR. However, the field dependence of the MR is non-linear below $8 \mathrm{~T}$ and the crossover field is far too low. The high field MR data can be fitted to a simple power-law (equation 6.2). The exponent, $m$, should be 1 for linear behavior, but ranges from 1.5 at high temperatures to around $1.1-1.2$ at low temperatures.5. Hence, the MR in these compounds is unlikely to be caused by quantum linear MR and it seems that a three-carrier model is more appropriate as shown by the fits to the $\mathrm{SrFe}_{2} \mathrm{As}_{2}$ samples. There is a MR up to $1600 \%$ observed for the superconducting samples of $\mathrm{SrFe}_{2} \mathrm{As}_{2}$ at $2 \mathrm{~K}$ and $8 \mathrm{~T}$. This enhancement is most likely due to three-carrier transport in an inhomogeneous system with superconducting and metallic regions, and

\footnotetext{
${ }^{5}$ only for non-superconducting samples
} 
where the mobilities are significantly enhanced. 


\section{Chapter 7}

\section{The effect of electronic doping on the magnetic, electronic and vibrational properties of $\mathrm{FeSr}_{2} \mathrm{YCu}_{2} \mathrm{O}_{6+\mathrm{y}}$}

\subsection{Introduction}

Another interesting approach to study the effect of Fe in HTSC materials is replacing some of the $\mathrm{Cu}$ in the cuprates with Fe. A good candidate for such a replacement is on a derivative of a parent compound $\mathrm{YSr}_{2} \mathrm{Cu}_{3} \mathrm{O}_{6+\mathrm{y}}$ (YSCO), which is similar to $\mathrm{RuSr}_{2} \mathrm{RCu}_{2} \mathrm{O}_{6+y} \prod^{1}(\mathrm{Ru} 1212)$ and $\mathrm{RuSr}_{2} \mathrm{R}_{2-\mathrm{x}} \mathrm{Ce}_{\mathrm{x}} \mathrm{Cu}_{2} \mathrm{O}_{10-\mathrm{y}} 1$ $(\mathrm{Ru} 1222)^{2}$, where a fraction of the $\mathrm{Cu}$ is replaced by $\mathrm{Ru}$. This is interesting to study because Ru1212/Ru1222 are well studied compounds, which have shown coexistence between superconductivity arising from the $\mathrm{CuO}_{2}$ planes and magnetic order from the $\mathrm{RuO}_{2}$ planes [170, 223 227]. Furthermore, this kind of coexistence has not attracted much attention in $\mathrm{FeSr}_{2} \mathrm{YCu}_{2} \mathrm{O}_{6+\mathrm{y}}$ (Fe1212). Despite the high Fe content Yamaguchi et al. [228] have found a maximum superconducting transition temperature, $T_{\mathrm{C}}$, of $64 \mathrm{~K}$. Therefore

\footnotetext{
${ }^{1} \mathrm{R}$ is a rare earth element

${ }^{2}$ studied in the next chapter 8
} 
the Fe1212 system has a high potential to offer some additional information about the coexistence of superconductivity and magnetism. 


\subsection{Synthesis and structure}

Samples of Fe1212 were prepared by stoichiometric mixing of high purity amounts of $\mathrm{Y}_{2} \mathrm{O}_{3}, \mathrm{Sr}\left(\mathrm{NO}_{3}\right)_{2}, \mathrm{CuO}$ and $\mathrm{Fe}_{2} \mathrm{O}_{3}$. $\mathrm{CaO}, \mathrm{La}_{2} \mathrm{O}_{3}$ or $\mathrm{ZnO}$ were added as dopants, which leads to hole doping when $\mathrm{Y}$ atoms are replaced with Ca; electron doping when $\mathrm{Sr}$ is replaced by La; and a fast suppression of superconductivity by replacing just a few percent of $\mathrm{Cu}$ with $\mathrm{Zn}$. The powder was ground together and denitrated at $700{ }^{\circ} \mathrm{C}$ for $12 \mathrm{~h}$ before regrinding, pelleting and annealing at $1000{ }^{\circ} \mathrm{C}$ in air for $24 \mathrm{~h}$. Afterward they were treated in $\mathrm{N}_{2}$ at $700{ }^{\circ} \mathrm{C}$ for another $24 \mathrm{~h}$, which should ensure a migration of most of the $\mathrm{Fe}$ into the $\mathrm{Cu}(1)$-chain site. Finally, the as-prepared samples were oxygen loaded at $600^{\circ} \mathrm{C}$ and slowly cooled down to $350^{\circ} \mathrm{C}$. The last step could be done at ambient pressure to ensure full superconductivity contrary to the parent compound of $\mathrm{YSr}_{2} \mathrm{Cu}_{3} \mathrm{O}_{6+\mathrm{y}}$ where high pressure oxygen loading is necessary to ensure full superconductivity.

With this method samples of pure Fe1212, La and Ca doped samples with 0.1 and 0.2 , and samples with $0.01,0.02,0.03$ and $0.05 \mathrm{Zn}$ doping were produced. The quality of the samples was checked between individual steps as well as at the end via powder XRD measurements employing Co- $K_{\alpha}$ radiation (figureA.4, A.5, A.6, A.7 and A.8). These measurements showed phase-pure polycrystalline samples within the limit of detectability (less than $1 \%$ ). The structure of Fe1212 is shown in figure 7.1 which is very similar to $\mathrm{RuSr}_{2} \mathrm{RCu}_{2} \mathrm{O}_{6+\mathrm{y}}$ [226]. It contains two $\mathrm{CuO}_{2}$ planes and a $\mathrm{SrFeO}_{3-\mathrm{y}}$ subunit [170, 229]. 


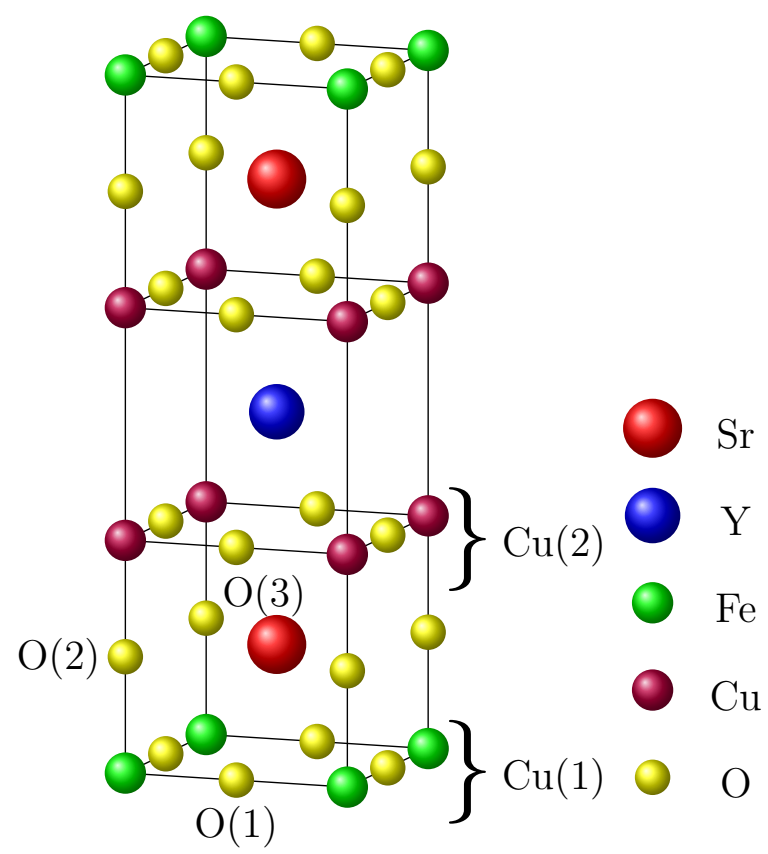

Figure 7.1: Crystal structure of $\mathrm{FeSr}_{2} \mathrm{YCu}_{2} \mathrm{O}_{6+\mathrm{y}}$ with $\mathrm{Fe}$ and $\mathrm{Cu}$ at their preferred position. 


\subsection{Magnetic properties}

Zero-field-cooled (ZFC) and field-cooled (FC) magnetization measurements were performed in an applied magnetic field of $2 \mathrm{mT}$. Figure 7.2 shows the results for undoped Fe1212, where a crossing towards negative magnetization for ZFC and FC is observed indicating the superconducting transition around a temperature of $64 \mathrm{~K}$.

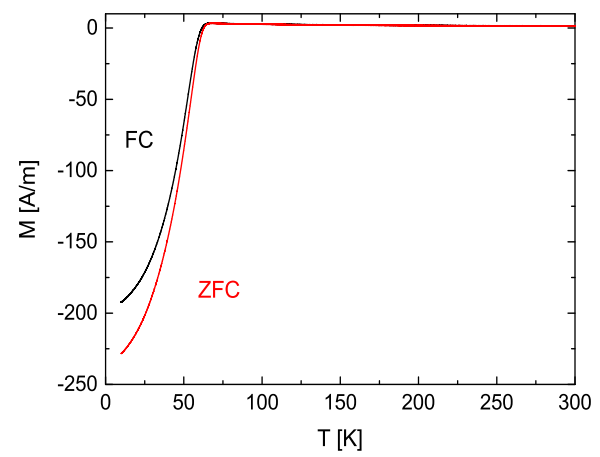

Figure 7.2: Temperature dependent magnetization for undoped Fe1212 at a magnetic field of $2 \mathrm{mT}$.

Similar measurements were done for all samples and the effect of electron and hole doping on $T_{\mathrm{C}}$ is shown in figure $7.3 \mathrm{a}$. Both dopings reduce $T_{\mathrm{C}}$ and superconductivity is completely suppressed at a doping of either $0.2 \mathrm{La}$ or Ca. Furthermore, figure $7.3 \mathrm{a}$ suggests that the optimal doping for this material is very close to the undoped compound. 


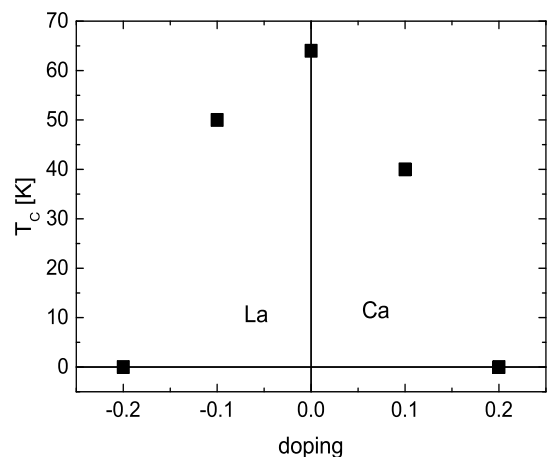

(a) Effect of electron (La) and hole (Ca) doping on $T_{\mathrm{C}}$.

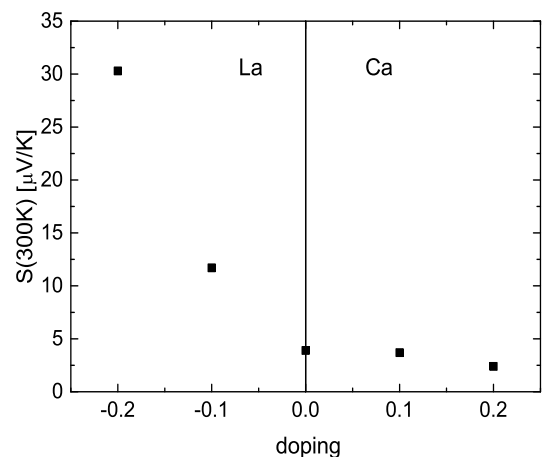

(b) Doping dependence of the room temperature thermopower.

Figure 7.3: Doping dependence of the superconducting transition temperature and room temperature thermopower in $\mathrm{FeSr}_{2} \mathrm{YCu}_{2} \mathrm{O}_{6+\mathrm{y}}$.

Such a superconducting dome is commonly observed in the HTSC cuprates, which usually also shows a decrease in the room temperature $(\sim 295 \mathrm{~K})$ thermopower value, $S(295 K)$, with increasing hole doping. This is indeed what was found in figure $7.3 \mathrm{~b}$. The overall $T_{\mathrm{C}}$ found here is significantly lower than that in the parent compound YSCO where it reaches a maximum of $80 \mathrm{~K}$ [230]. It is known that $\mathrm{Fe}$ on the $\mathrm{Cu}(2)$ site suppresses $T_{\mathrm{C}}$ in the $\mathrm{Y} 123$ compound via pair-breaking by around $5 \mathrm{~K} / \% \mathrm{Fe}$ [231 233]. It is likely that some iron remains on the $\mathrm{Cu}(2)$ site in $\mathrm{Fe} 1212$ and the amount can be estimated to be around $\sim 3 \%$ assuming a similar suppression rate.

Susceptibility measurements were undertaken at a field of 6 T. All samples show a Curie-Weiss $(\mathrm{CW})$ temperature dependence above $T_{\mathrm{C}}$ with a departure from the CW temperature dependent behavior when approaching $T_{\mathrm{C}}$, suggesting superconductivity is still not completely suppressed at $6 \mathrm{~T}$ as can be seen in figure 7.4. This is also an indication that the upper critical field, $B_{\mathrm{c} 2}$, has to be much higher than the applied magnetic field of $6 \mathrm{~T}$. 


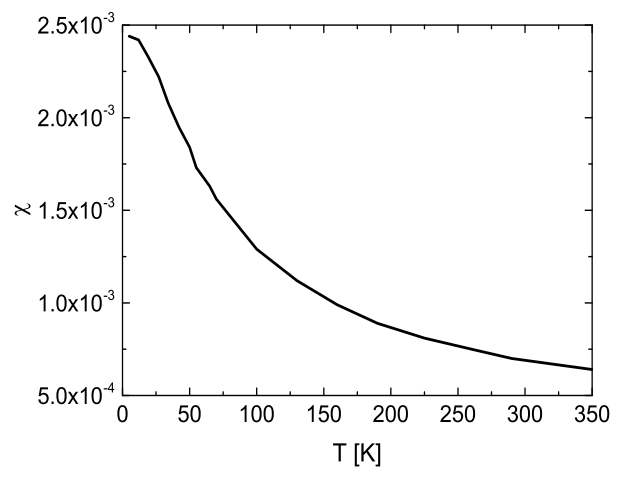

(a) $\chi$

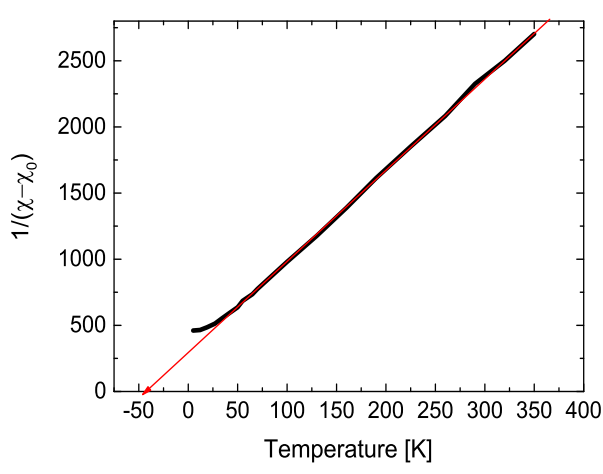

(b) $1 / \chi-\chi_{0}$

Figure 7.4: Temperature dependence of the a) susceptibility and b) $1 / \chi_{-} \chi_{0}$ for $\mathrm{Fe} 1212$.

The departure from the CW temperature dependent behavior can be clearly seen in figure $7.4 \mathrm{~b}$ at $50 \mathrm{~K}$, and the fitting to equation 3.3 was done above this departure in order to avoid any contributions from superconductivity or magnetic order. Noticeable was a large increase in the temperature independent part of the susceptibility, $\chi_{0}$, for all superconducting samples, which shows a similar doping behavior as $T_{\mathrm{C}}$. The values for $\chi_{0}$ are shown in figure $7.5 \mathrm{a}$, where this behavior might originate from a Stoner enhancement or an effective mass effect. 


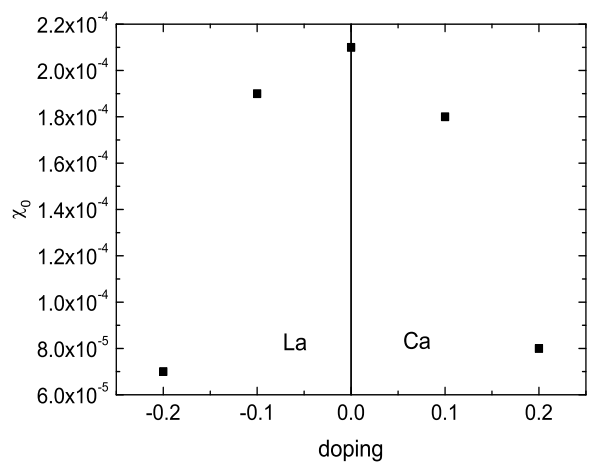

(a) Doping dependence of $\chi_{0}$.

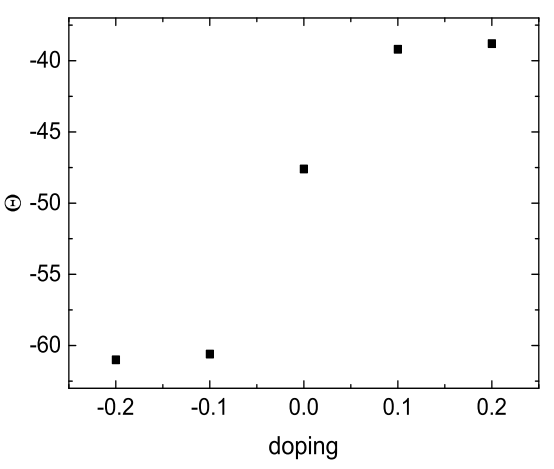

(b) Doping dependence of the CW temperature, $\Theta$.

Figure 7.5: Doping dependence of the fit parameters.

Furthermore, there is a decrease in the magnitude of the CW temperature with increasing hole doping, which is shown in figure $7.5 \mathrm{~b}$. The negative sign indicates a dominant antiferromagnetic exchange interaction, which becomes weaker with increasing hole doping.

The effective moment per formula unit, $P_{\text {eff }}^{\text {f.u. }}$, was calculated from the CW constant, $C$ (equation 3.5). Similar values were obtained for all superconducting samples (figure 7.6), and it is the highest for 0.2 La and the lowest for $0.2 \mathrm{Ca}$ doping. A value of $P_{\mathrm{eff}}^{\mathrm{f} . \mathrm{u}}=3.4 \mu_{\mathrm{B}}$ was obtained for the superconducting samples, which is in good agreement with other measurements on Fe1212 by Pissas et al. [234]. The values for different spin configurations of Fe are shown in table 7.1 and these suggest that there is a mixture of at least two different spin configurations. Mössbauer measurements on the related compound of $\mathrm{FeBa}_{2} \mathrm{YCu}_{2} \mathrm{O}_{6+\mathrm{y}}$ by Felner et al. and on $\mathrm{FeSr}_{2} \mathrm{YCu}_{2} \mathrm{O}_{6+\mathrm{y}}$ by Hata et al. [14, 235] have shown that the Fe ions at the $\mathrm{Cu}(2)$ site are always in the high spin $\left(S=5 / 2 \rightarrow 5.92 \mu_{\mathrm{B}}\right)$ configuration of $\mathrm{Fe}^{3+}$. The Fe on the $\mathrm{Cu}(1)$ site was found to be in two different states which are the high- and the low-spin configurations of $\mathrm{Fe}^{3+}$ [235]. There is no $\mathrm{Cu}$ contribution because the $\mathrm{Cu}$ plane is conducting and hence it has no static moment. Then it is 
possible to calculated the amount of Fe in the different spin states $\mathrm{s}^{3}$ from,

$$
P_{\text {f.u. }}^{2}=x P_{\mathrm{s} 1}^{2}+(1-x) P_{\mathrm{s} 2}^{2}
$$

where $P_{\text {f.u. }}$ is the effective moment per formula unit and $\mathrm{x}$ is the amount of Fe in the spin configuration $s 1\left(P_{\mathrm{s} i}\right.$ are the effective moments of Fe in the spin configuration si). Equation 7.1 can be rewritten for $\mathrm{x}$ as,

$$
x=\frac{P_{\text {f.u. }}^{2}-P_{\mathrm{s} 2}^{2}}{P_{\mathrm{s} 1}^{2}-P_{\mathrm{s} 2}^{2}}
$$

and by assuming that there is a high spin $\left(S=5 / 2 \rightarrow 5.92 \mu_{\mathrm{B}}\right)$ and a low spin $\left(S=1 / 2 \rightarrow 1.73 \mu_{\mathrm{B}}\right)$ configuration yielding $27 \%$ Fe in the high spin state and $73 \%$ in the low spin state. Hata et al. 235] have found a distribution of $60 \%$ low-spin and $40 \%$ high-spin $\mathrm{Fe}^{3+}$ from Mössbauer measurements, which results in an effective moment for the whole compound of $P_{\text {eff }}^{\text {f.u. }}=3.98 \mu_{\mathrm{B}}$. Such a difference can occur through the redistribution of Fe from the $\mathrm{Cu}(2)$ site to the $\mathrm{Cu}(1)$ site as it has in our sample.

\begin{tabular}{|l|c|c|}
\hline & $S$ & $P_{\text {eff }}^{\mathrm{Fe}}\left[\mu_{\mathrm{B}}\right]$ \\
\hline \hline $\mathrm{Fe}^{2+}$ & 2 & 4.99 \\
\hline $\mathrm{Fe}^{3+}$ & $5 / 2$ & 5.92 \\
\hline $\mathrm{Fe}^{3+}$ & $3 / 2$ & 3.87 \\
\hline $\mathrm{Fe}^{3+}$ & $1 / 2$ & 1.73 \\
\hline $\mathrm{Fe}^{4+}$ & 2 & 4.99 \\
\hline $\mathrm{Fe}^{4+}$ & 1 & 2.83 \\
\hline
\end{tabular}

Table 7.1: The effective moment of different spin configurations of Fe.

\footnotetext{
${ }^{3} \mathrm{Fe}$ on the two different sites in the two different spin states behaves as completely different magnetic Ions
} 


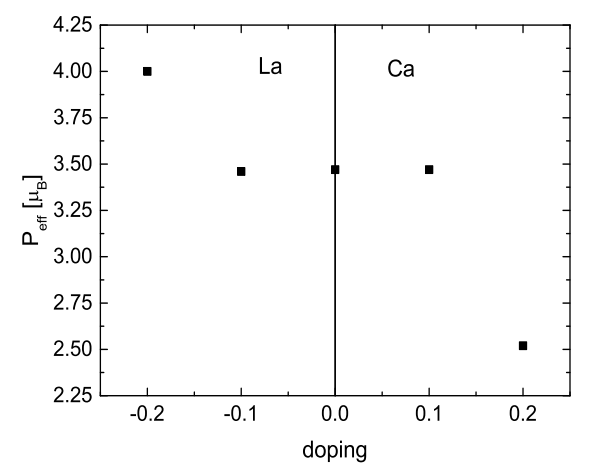

Figure 7.6: Doping dependence of the effective moment per formula unit. 


\subsection{Raman Spectroscopy}

Raman spectroscopy was carrier out on doped and undoped Fe1212 samples, and a typical example of a room temperature spectrum for an undoped compound is shown in figure 7.7. All samples show a variety of Raman peaks in the spectra, which are interpreted through comparisons with similar samples like $\mathrm{YBa}_{2} \mathrm{Cu}_{3} \mathrm{O}_{7+\mathrm{y}}$ [236 239], $\mathrm{FeSr}_{2} \mathrm{Y}_{2-\mathrm{x}} \mathrm{Ce}_{\mathrm{x}} \mathrm{Cu}_{2} \mathrm{O}_{10-\mathrm{y}}$ [240] and $\mathrm{RuSr}_{2} \mathrm{GdCu}_{2} \mathrm{O}_{8}$ [241].

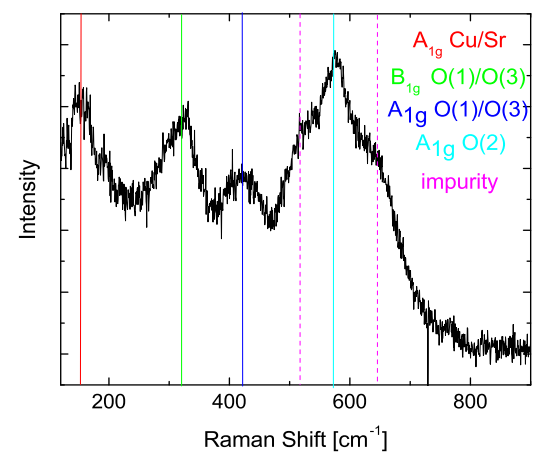

Figure 7.7: Room temperature Raman spectrum of an undoped Fe1212 sample.

The assignment is presented starting from the low frequency side. The red line at around $150 \mathrm{~cm}^{-1}$, can be attributed to an $A_{1 g}$ mode involving either $\mathrm{Cu}(2)$ motion or $\mathrm{Sr}$ vibrations both along the z-axis. These two modes have the same symmetry and hence overlap. They are expected to interact and split, which results in the small difference observed in our spectra. A $B_{1 g}$ mode from out-of-phase vibration of the $\mathrm{O}(1) / \mathrm{O}(3)$ is assigned to the green line at $320 \mathrm{~cm}^{-1}$. The feature around $400 \mathrm{~cm}^{-1}$ to $450 \mathrm{~cm}^{-1}$ in blue is attributed to an $A_{1 g}$ mode in-phase $\mathrm{O}(1) / \mathrm{O}(3)$ motion. The cyan line around $575 \mathrm{~cm}^{-1}$ is identified as an $A_{1 g}$ mode from the apical oxygen $\mathrm{O}(2)$. This line also has features at the lower and higher frequency side (shown in figure 7.7 and 7.8 as the pink lines), which are most likely caused by impurities. None of the modes show much frequency shift with doping probably because the

\footnotetext{
${ }^{4}$ see figure 7.1 for the position of $\mathrm{Cu}$ and $\mathrm{O}$
} 
resolution is not good enough. Only the lower frequency feature (pink line) for the apical oxygen $\mathrm{O}(2)$ displays lower frequency values with doping on either side of the undoped parent compound.

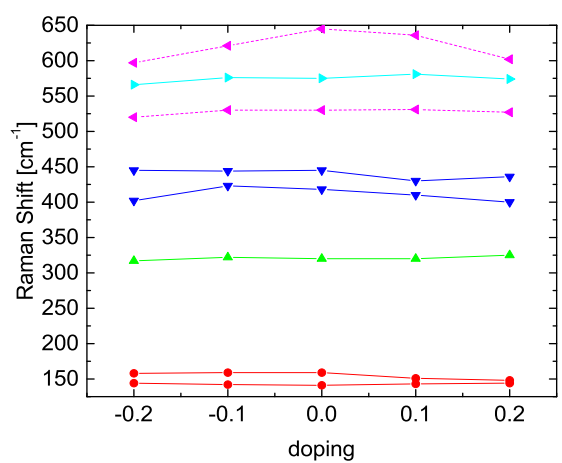

Figure 7.8: Doping dependence of the Raman peaks with electron and hole doping. 


\subsection{The effects of $\mathrm{Zn}$ doping}

The idea behind adding $\mathrm{Zn}$ is to suppress superconductivity and investigate any possible remaining magnetic order, which might previously be hidden. Such a possibility is supported by the observed coexistence of magnetism and superconductivity in the Ru1212 [224 226, 242] compound, where the magnetism is from the $\mathrm{RuO}_{2}$ planes. Furthermore, a magnetic ordering is observed in $\mathrm{FeSr}_{2} \mathrm{Y}_{2-\mathrm{x}} \mathrm{Ce}_{\mathrm{x}} \mathrm{Cu}_{2} \mathrm{O}_{10-\mathrm{y}}$ (Fe1222), which will be described in chapter 8 . The doping range of $\mathrm{x}=0.01-0.05$ was chosen because with lower $\mathrm{Zn}$ values one would only partially suppress superconductivity, which might offer some additional insights into the superconducting pair-breaking mechanism and furthermore allows an estimation of the suppression rate per Zn doping.

Figure 7.9 shows the results from ZFC and FC measurements where it can be seen that superconductivity is gradually suppressed by Zn doping. While the samples with a Zn content of $\mathrm{x}=0.01,0.02$ and 0.03 are still superconducting with $T_{\mathrm{C}}$ around $30 \mathrm{~K}, 20 \mathrm{~K}$ and $12 \mathrm{~K}$ respectively, the sample with $\mathrm{x}=0.05$ is not superconducting anymore. However, the ZFC still shows a transition (peak) at around $64 \mathrm{~K}$ plus a separation between the $\mathrm{ZFC}$ and FC traces at this temperature, which indicates that a certain fraction of this sample might still be superconducting, since this is very close to the optimal $T_{\mathrm{C}}$ for the undoped sample [243]. Hence, it is most likely to arisen from an inhomogeneous distribution of $\mathrm{Zn}$ rather than magnetic order. 


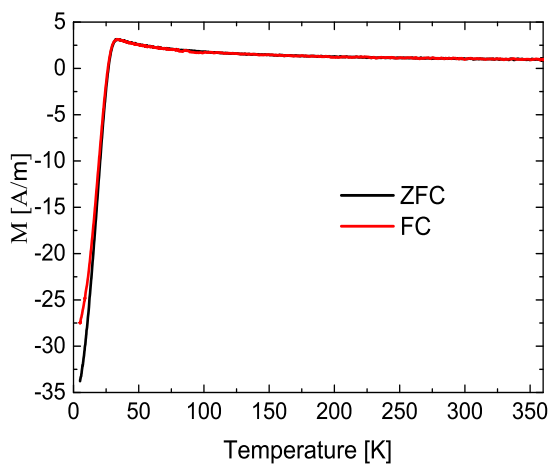

(a) $\mathrm{x}=0.01$

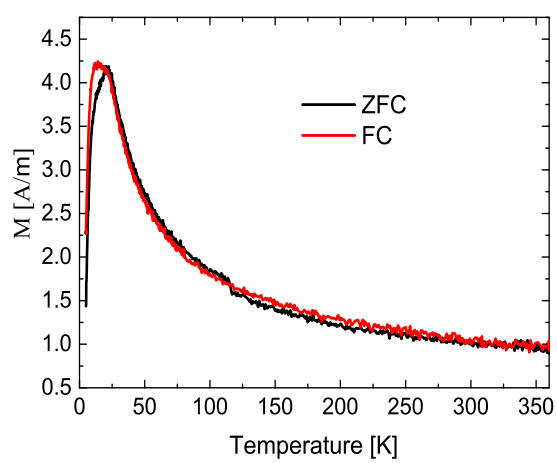

(c) $\mathrm{x}=0.03$

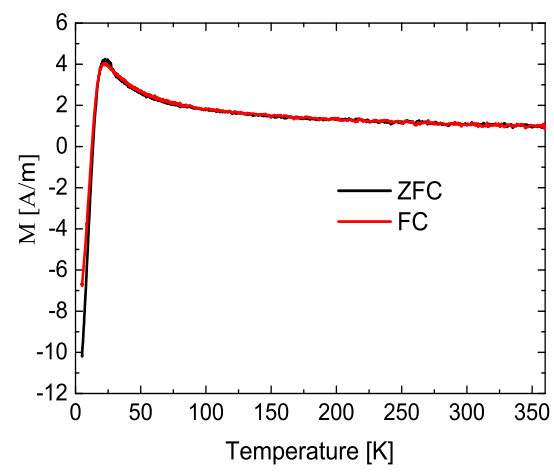

(b) $\mathrm{x}=0.02$

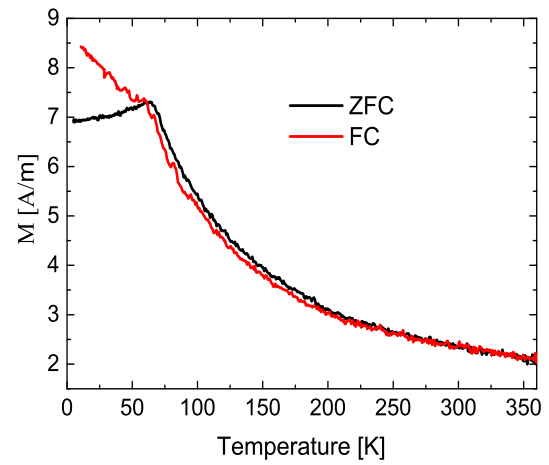

(d) $\mathrm{x}=0.05$

Figure 7.9: The temperature dependence of ZFC and FC magnetization of $\mathrm{FeSr}_{2} \mathrm{YCu}_{2-\mathrm{x}} \mathrm{Zn}_{\mathrm{x}} \mathrm{O}_{6+\mathrm{y}}$ in an applied field of $2 \mathrm{mT}$.

For YBCO it has been found that the suppression rate, $d T_{C} / d x$, depends on the hole concentration in the initial region, where the suppression is roughly linear. This effect is related to the size of the normal state pseudogap [115, 244. Figure 7.10 shows $T_{\mathrm{C}}$ versus $\mathrm{Zn}$ content for Fe1212 as well as for optimally doped (dashed blue line) and heavily underdoped (red solid line) YBCO [244]. The suppression rate of $10 \mathrm{~K} / \% \mathrm{Fe}$ found here for Fe1212 is too high for a Zn pair breaking pathway known in the cuprates. The most likely reason is that it is due to more $\mathrm{Fe}$ on the $\mathrm{Cu}(2)$ site, which leads to additional Fe pair breaking not appearing in the bulk sample. This also explains why the initial $T_{\mathrm{C}}$ is lower than expected for the $\mathrm{x}=0.01 \mathrm{Zn}$ doping and results in 
an overall reduced $T_{\mathrm{C}}$.

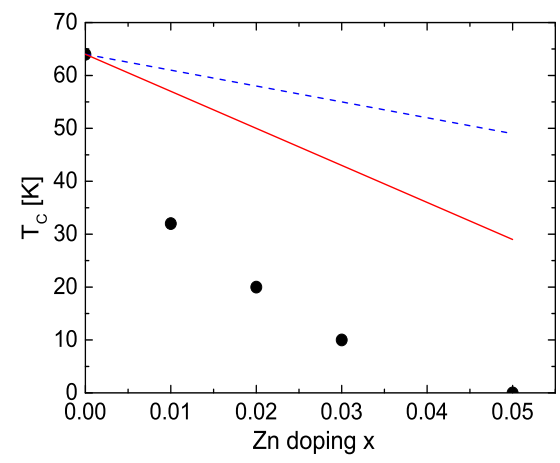

Figure 7.10: The superconducting transition temperature, $T_{\mathrm{C}}$, against the Zn doping in Fe1212 (black circles). Also shown are the suppression rate for optimally doped (dashed blue line) and heavily underdoped (solid red line) YBCO [244] based on the initial $T_{\mathrm{C}}$ of $64 \mathrm{~K}$. 


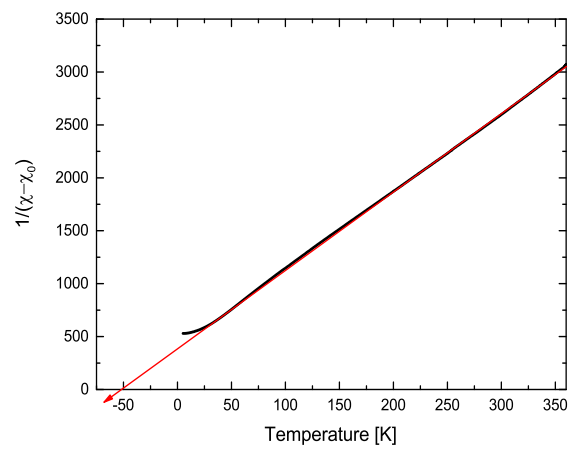

(a) $\mathrm{x}=0.01$

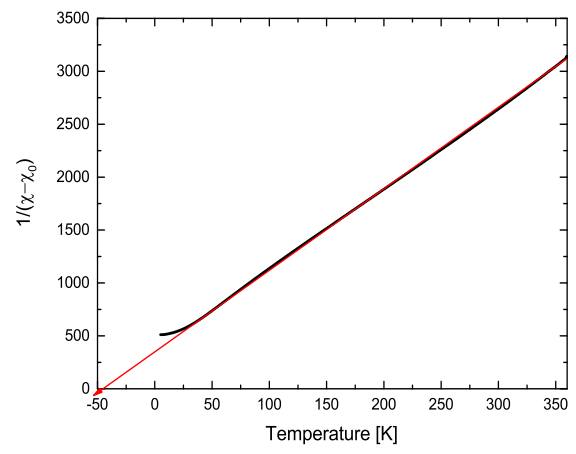

(c) $\mathrm{x}=0.03$

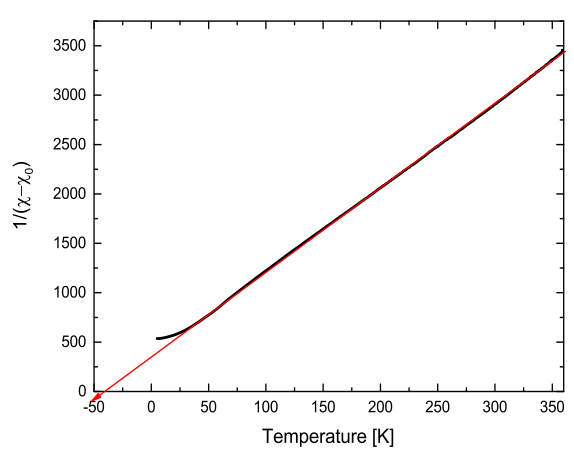

(b) $\mathrm{x}=0.02$

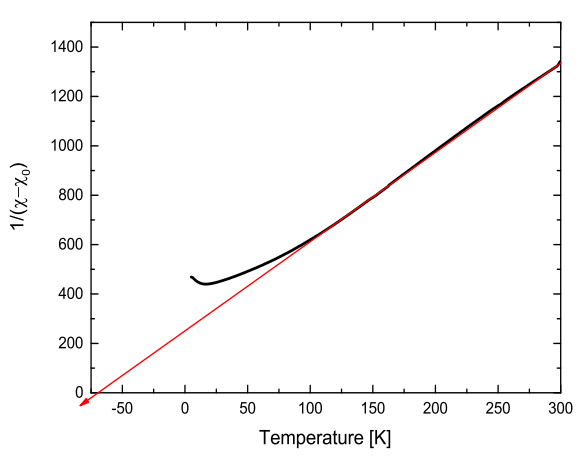

(d) $\mathrm{x}=0.05$

Figure 7.11: $1 /\left(\chi-\chi_{0}\right)$ plot for Fe1212 samples together with the linear fit for a $\mathrm{CW}$ temperature dependence.

Susceptibility measurements were undertaken in a magnetic field of $6 \mathrm{~T}$. Figure 7.11 displays plots of $1 /\left(\chi-\chi_{0}\right)$ for all four Zn doped samples. All samples show a CW temperature dependent behavior down to $40 \mathrm{~K}$ except for the sample with $0.05 \mathrm{Zn}$, which already shows a departure at a higher temperature of $110 \mathrm{~K}$. The resultant $\mathrm{CW}$ temperature, $\Theta$, the effective moment per formula unit, $P_{\text {eff }}^{\text {f.u. }}, \chi_{0}$ and $S(295 K)$ are listed in table 7.2 . 


\begin{tabular}{|c|c|c|c|c|c|}
\hline Zn doping & $T_{\mathrm{C}}[\mathrm{K}]$ & $\Theta[\mathrm{K}]$ & $P_{\text {eff }}^{\text {f.u. }}\left[\mu_{\mathrm{B}}\right]$ & $\chi_{0}$ & $S(295 \mathrm{~K})\left[\mathrm{\mu V} \mathrm{K}^{-1}\right]$ \\
\hline \hline 0.01 & $30(2)$ & $-50(2)$ & 2.72 & $2.5 \mathrm{e}-4$ & $10(1)$ \\
\hline 0.02 & $20(2)$ & $-48(2)$ & 2.54 & $2.7 \mathrm{e}-4$ & $11(1)$ \\
\hline 0.003 & $10(2)$ & $-47(2)$ & 2.67 & $2.6 \mathrm{e}-4$ & $12(1)$ \\
\hline 0.05 & $\mathrm{~d}^{5}$ & $-69(3)$ & 3.91 & $9.5 \mathrm{e}-6$ & $39(2)$ \\
\hline
\end{tabular}

Table 7.2: $T_{C}, \Theta, P_{\text {eff }}^{\text {f.u. }}, \chi_{0}$ and $S(295 \mathrm{~K})$ of Zn doped Fe1212.

The values of $S(295 \mathrm{~K})$ are nearly the same from $x=0.01$ to $x=0.03$ but they are significantly higher for $x=0.05$. Comparing the observed values for the $x=0.05$ sample to the $S(295 \mathrm{~K})$ values found in the hole doped cuprates [115] suggests that the sample is significantly underdoped. The CW temperature and the temperature independent part of the susceptibility are nearly the same for $x=0.01$ to 0.03 and these values are similar to the undoped

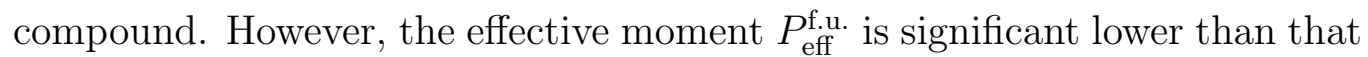
previously observed. This can be the case, if there is more Fe in the low spin state in these samples. There is no evidence for a Zn-induced local moment at these concentrations as it is seen in the superconducting cuprates [245]. The Curie-Weiss temperature is more negative and the effective moment is larger for $x=0.05$. This may be due to a higher amount of disorder in the sample and a larger fraction of $\mathrm{Fe}$ on the $\mathrm{Cu}(2)$ site.

Field loop measurements were performed at $5 \mathrm{~K}$ as shown in figure 7.12 for $x=0.01-0.03$ and $x=0.05$. The small initial decrease in the moment per gram, $M$, in the low-field region of the field loops for $x=0.01$ and $x=0.02$ is consistent with the presence of superconductivity that is seen in figure $7.12 \mathrm{a}$ and $7.12 \mathrm{~b}$, The positive part is a contribution from paramagnetic Fe ions. There is no initial decrease in $M$ for the $x=0.03$ and $x=0.05$ samples and there is hysteresis observed up to $6 \mathrm{~T}$. However, hysteresis is also observed up to $6 \mathrm{~T}$ for the superconducting samples with less $\mathrm{Zn}$ and hence it is not clear if the hysteresis observed for $x=0.05$ is due to some superconducting regions with lower $x$ or due to magnetic ordering. More measurements are required at higher temperatures for $\mathrm{x}=0.05$ and more homogeneous samples are required where superconductivity is unambiguously suppressed and to determine whether there is magnetic ordering. 


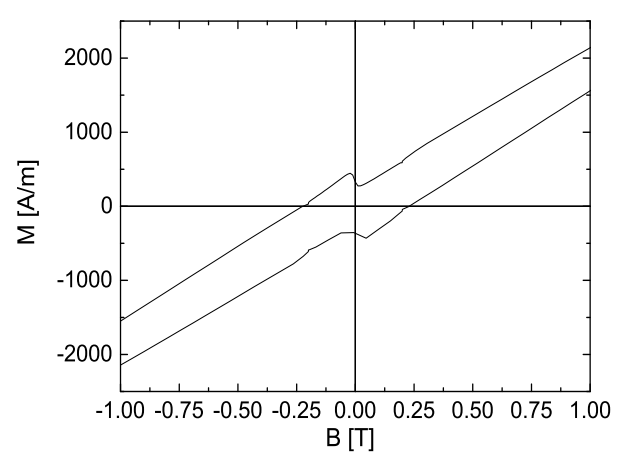

(a) $\mathrm{x}=0.01$

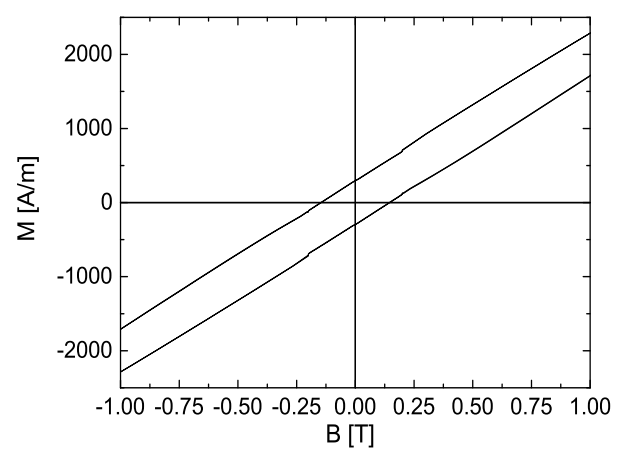

(c) $\mathrm{x}=0.03$

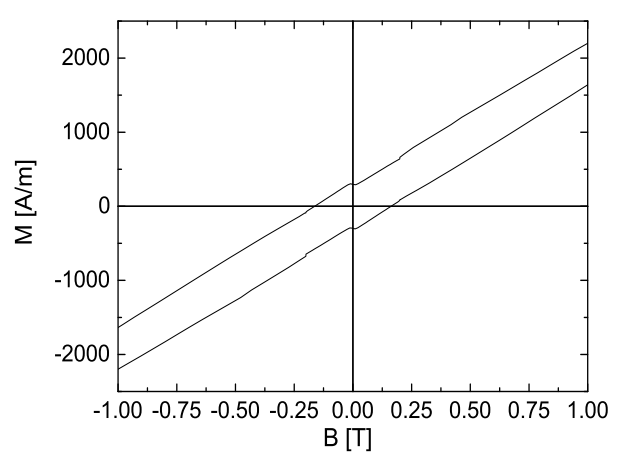

(b) $\mathrm{x}=0.02$

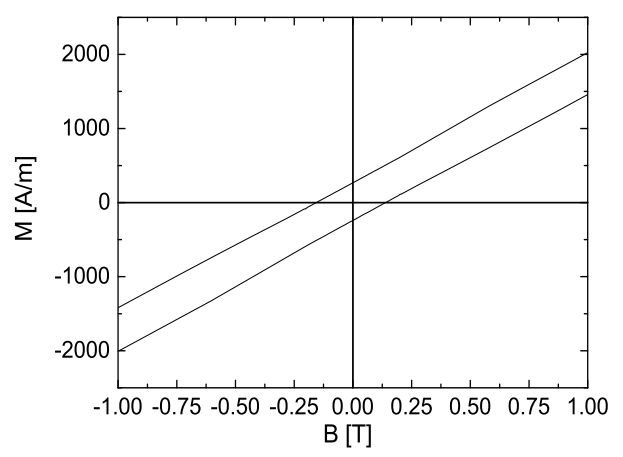

(d) $\mathrm{x}=0.05$

Figure 7.12: Low field magnetization loops at 5K for Fe1212 with different Zn content. 


\subsection{Summary and conclusions}

$\mathrm{FeSr}_{2} \mathrm{YCu}_{2} \mathrm{O}_{6+\mathrm{y}}$ (Fe1212) was investigated by magnetic and magnetotransport measurements with different dopants (La, Ca, Zn). A maximum $T_{\mathrm{C}}$ of $64 \mathrm{~K}$ was found for the undoped parent compound, which is smaller than the iron free compound of $\mathrm{YSr}_{2} \mathrm{Cu}_{3} \mathrm{O}_{6+\mathrm{y}}$ (YSCO) which has a $T_{\mathrm{C}}$ of around $80 \mathrm{~K}$ [230]. Fe1212 should have a similar $T_{\mathrm{C}}$, if all the irons are at the $\mathrm{Cu}(1)$ sites. It is known that $\mathrm{Fe}$ on the $\mathrm{Cu}(2)$ site suppresses $T_{\mathrm{C}}$ in the $\mathrm{Y} 123$ compound via pair-breaking by around $5 \mathrm{~K} / \% \mathrm{Fe}$ [231 233] and hence the remaining iron on the $\mathrm{Cu}(2)$ site can be estimated to be around $\sim 3 \%$. A possible Stoner enhancement was observed in the temperature independent part of the susceptibility. Furthermore, through the CW analysis, a decrease in the antiferromagnetic exchange interaction with increasing hole doping is observed as well as a $\mathrm{Fe}^{3+}$ high spin configuration for the $\mathrm{Cu}(2)$ site and a mixture of $\mathrm{Fe}^{3+}$ in the high and low spin configuration for the $\mathrm{Cu}(1)$ site.

$\mathrm{Zn}$ is found to be successful in suppressing superconductivity above $x=$ 0.03. However, the rate of suppression, $\triangle T_{C} / x(Z n)$, is found to be high, which suggests the pair breaking is unlikely to come from $\mathrm{Zn}$ alone. There is an additional suppression due to an increased Fe content on the $\mathrm{Cu}(2)$ site in comparison to the undoped sample. The samples with reduced superconducting transition temperatures $x=0.01-0.03$ show similar values of $S(295 \mathrm{~K})$, $\Theta, \chi_{0}$ and $P_{\text {eff }}$, while a significant difference in those values was observed for the sample with $x=0.05$. There is also a noticeable magnetization versus applied field in all samples up to $6 \mathrm{~T}$, but the origin of this hysteresis and also the nature of this magnetic order are unclear.

More measurements with better homogenized samples are therefore needed to clarify the origin of the transition in the $x=0.05$ sample. Such samples should prevent contribution from small fractions of the sample with significant different doping. Hence, is should be possible to identify the origin of the transition in the ZFC and FC traces for the $x=0.05$ sample. Additional measurements at higher temperatures will then make it possible to determine the underlying magnetic ordering. 


\section{Chapter 8}

\section{Magnetic and}

\section{magnetotransport properties of $\mathrm{FeSr}_{2} \mathrm{Y}_{2-\mathrm{x}} \mathrm{Ce}_{\mathrm{x}} \mathrm{Cu}_{2} \mathrm{O}_{10-\mathrm{y}}$ : Spin and electronic disorder}

\subsection{Introduction}

This chapter presents the results from magnetic and magnetotransport measurements on $\mathrm{FeSr}_{2} \mathrm{Y}_{2-\mathrm{x}} \mathrm{Ce}_{\mathrm{x}} \mathrm{Cu}_{2} \mathrm{O}_{10-\mathrm{y}}$ (Fe1222). This material offers valuable additional insights into the properties of iron-based superconductors, because it uses the same building blocks as the high temperature superconducting cuprates and is very similar to $\mathrm{RuSr}_{2} \mathrm{R}_{2-\mathrm{x}} \mathrm{Ce}_{\mathrm{x}} \mathrm{Cu}_{2} \mathrm{O}_{10-\mathrm{y}}$ [246] ( $\mathrm{Ru} 1222$ ) ( $\mathrm{R}$ is a rare earth) and $\mathrm{FeSr}_{2} \mathrm{YCu}_{2} \mathrm{O}_{6+\mathrm{y}}(\mathrm{Fe} 1212)$ described in chapter 7. Fe1222 has not shown any sign of superconductivity and hence offers a good option to study the underlying magnetic properties without the influence from other competing order mechanisms. It might also offer an explanation to why this system is not superconducting and how it is suppressed. 


\subsection{Synthesis and structure}

Polycrystalline $\mathrm{FeSr}_{2} \mathrm{Y}_{2-\mathrm{x}} \mathrm{Ce}_{\mathrm{x}} \mathrm{Cu}_{2} \mathrm{O}_{10-\mathrm{y}}$ samples were synthesized using a standard solid state reaction method. Stoichiometric amounts of $\mathrm{Fe}_{2} \mathrm{O}_{3}, \mathrm{Sr}\left(\mathrm{NO}_{3}\right)_{2}$, $\mathrm{Y}_{2} \mathrm{O}_{3}, \mathrm{CuO}$ and $\mathrm{CeO}_{2}$ were ground together and the mixed powder was first denitrated at $700{ }^{\circ} \mathrm{C}$ on gold foil, then reground and pressed into pellets. The pellets were then sintered in air at $1050{ }^{\circ} \mathrm{C}$ for $24 \mathrm{~h}$. From here two different routes were taken. In the first attempt the pellets were oxygen loaded at 10 bar at a temperature of $550^{\circ} \mathrm{C}$ for $12 \mathrm{~h}$ and then slowly cooled down to room temperature over three days (type1). It became clear later on that it is not necessary to do the oxygen loading at higher pressure and the oxygen loading step could be done at ambient pressure with the same results. However those samples are not superconducting, which was also reported in some earlier reports on particular doping [223, 240, 247, 248]. From Mössbauer measurements it looks like Fe is nearly evenly distributed between the $\mathrm{CuO}_{2}$ plane and the $\mathrm{CuO}_{1-\mathrm{y}}$ chains. Therefore Fe induced pair breaking is very likely the cause of the non-existent superconductivity in this material. Hence, a different approach was taken in the 2nd method to force as much Fe from the $\mathrm{CuO}_{2}$ planes into the chains. To achieve such a re-positioning of Fe and $\mathrm{Cu}$ an intermediate step was introduced, where the pellets were heated to $750^{\circ} \mathrm{C}$ in an Ar atmosphere and subsequently quenched in liquid $\mathrm{N}_{2}$. After this procedure the samples are left with many oxygen deficiencies and it is possible to study the effect of oxygen doping between these oxygen reduced (OR) samples (type2 - OR) and those with a final oxygen loading step in a $\mathrm{O}_{2}$-atmosphere at ambient pressure, which leads to oxygen saturated samples (type2 - OS).

The quality of the samples was checked between individual steps as well as at the end with powder XRD measurements employing Co- $K_{\alpha}$ radiation. These measurements showed phase-pure polycrystalline samples within the limit of detectability (less than 1\%) (figure A.9, A.10, A.11 and A.12. The effects of oxygen loading as well as the additional annealing step in Ar-atmosphere could be seen in the changes in thermoelectric power (thermopower) (see table 8.1). 


\begin{tabular}{|c|c|c|c|}
\hline sample & $S_{\text {initial }}{ }^{1}\left[\mathrm{\mu V} \mathrm{K}^{-1}\right]$ & $S_{\mathrm{OR}}{ }^{2}\left[\mathrm{pVK}^{-1}\right]$ & $S_{\mathrm{OS}}{ }^{3}\left[\mathrm{p} \mathrm{K}^{-1}\right]$ \\
\hline \hline 0.2 & $67(1)$ & 4 & $29(1)$ \\
\hline 0.3 & $66(1)$ & $42 \overrightarrow{4(1)}$ & $34(1)$ \\
\hline 0.5 & $65(5)$ & $150(3)$ & $19(1)$ \\
\hline 0.6 & $67(1)$ & $197(2)$ & $29(1)$ \\
\hline 0.7 & $68(1)$ & $345(5)$ & $28(1)$ \\
\hline 0.9 & $72(2)$ & $350(10)$ & $39(1)$ \\
\hline
\end{tabular}

Table 8.1: The thermopower for Fe1222 samples at different processing steps.

The unit cell of Fe1222 has a fluorite block that is similar to the $\mathrm{R}_{2-\mathrm{x}} \mathrm{Ce}_{\mathrm{x}} \mathrm{CuO}_{4}$ electron-doped superconducting cuprates that have $\mathrm{CuO}_{2}$ planes. The fluorite blocks are separated by perovskite structures of $\mathrm{FeSrO}_{3-\mathrm{y}}$. The similar compound of Ru1222 is superconducting for sufficient oxygen loading with a value of $\mathrm{x}$ close to zero and it displays the coexistence of superconductivity from the $\mathrm{CuO}_{2}$ planes and magnetic order from the $\mathrm{RuO}_{2}$ planes [246]. However the $\mathrm{FeO}_{2-\mathrm{y}}$ plane in Fe1222 is considerably oxygen deficient with $y \sim 0.8$ in oxygen loaded samples [248]. The overall structure is shown in figure 8.1.

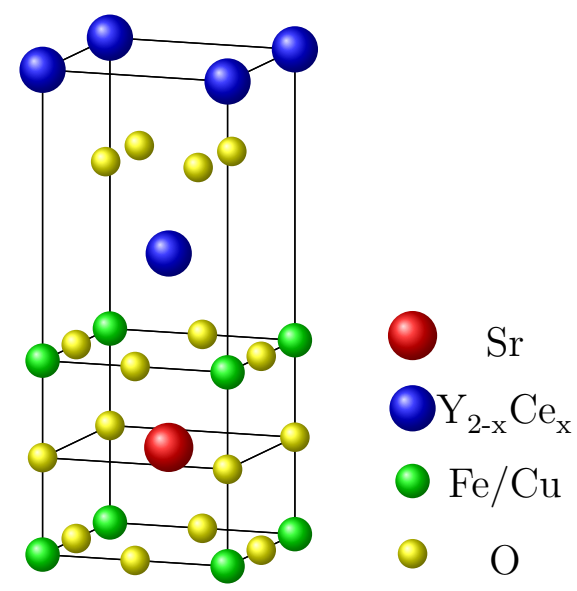

Figure 8.1: Crystal structure of $\mathrm{FeSr}_{2} \mathrm{Y}_{2-\mathrm{x}} \mathrm{Ce}_{\mathrm{x}} \mathrm{Cu}_{2} \mathrm{O}_{10-\mathrm{y}}$. 


\subsection{Magnetization}

Zero-field-cooled (ZFC) and field-cooled (FC) magnetization measurements were performed in an applied magnetic field of $2 \mathrm{mT}$. Figure 8.2 shows the results from these measurements, where a peak in the ZFC magnetization is observed at around a temperature of $20 \mathrm{~K}$. No indication was found for any kind of high temperature $\mathrm{Cu}$ ordering. Magnetization measurements on $\mathrm{FeSr}_{2} \mathrm{Y}_{1.5} \mathrm{Ce}_{0.5} \mathrm{Cu}_{2} \mathrm{O}_{10-\mathrm{y}}$ by Pissas et al. [240] show a peak in the ZFC magnetization was observed at low temperature. It was not clear if this peak is caused by a long range antiferromagnetic order or a spin-glass transition. Later on the same peak was observed be Felner et al. [223], who interpreted it as an antiferromagnetic order from $\mathrm{Cu}$ moments frustrated by $\mathrm{Fe}$ on the $\mathrm{Cu}$ sites or $\mathrm{Cu}$ on the Fe site. Such an ordering is known to occur in oxygen reduced samples around $350 \mathrm{~K}$. Felner et al. [223] argued that increasing the oxygen content would decrease the ordering temperature of the $\mathrm{Cu}$ moments.

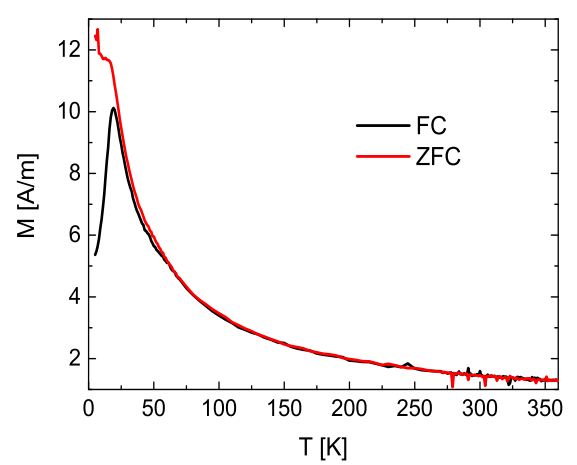

Figure 8.2: Temperature dependence of the ZFC and FC magnetization for $\mathrm{FeSr}_{2} \mathrm{Y}_{1.3} \mathrm{Ce}_{0.7} \mathrm{Cu}_{2} \mathrm{O}_{10-\mathrm{y}}$ at an applied field of $2 \mathrm{mT}$.

However, the above DC magnetization does not clarify the origin of the ZFC peak. AC magnetization measurements were performed for that purpose around the low temperature magnetic transition. A spin-glass transition would show a shift in the real part of the ac magnetization $M^{\prime}$ to higher temperature with increasing frequency. Figure 8.3 shows the results for three different Ce doping states, where a shift in the peak magnetization with $\mathrm{AC}$ 
field is observed.

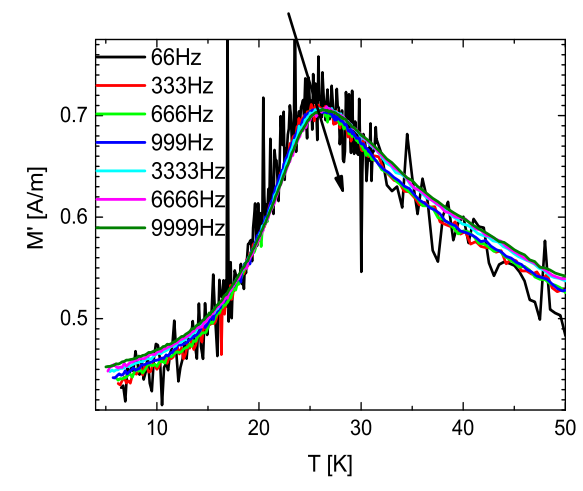

(a) $\mathrm{FeSr}_{2} \mathrm{Y}_{1.7} \mathrm{Ce}_{0.3} \mathrm{Cu}_{2} \mathrm{O}_{10-\mathrm{y}}$

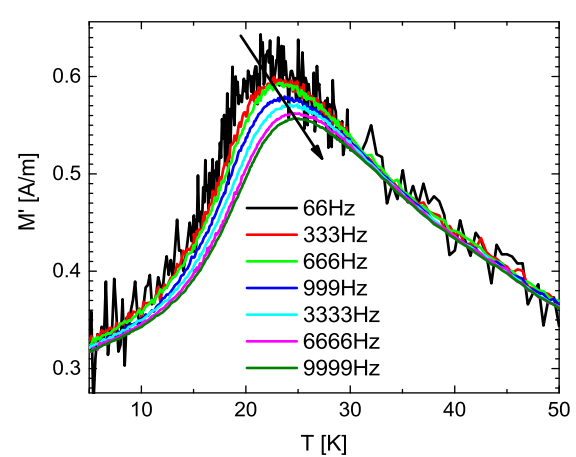

(b) $\mathrm{FeSr}_{2} \mathrm{Y}_{1.3} \mathrm{Ce}_{0.7} \mathrm{Cu}_{2} \mathrm{O}_{10-\mathrm{y}}$

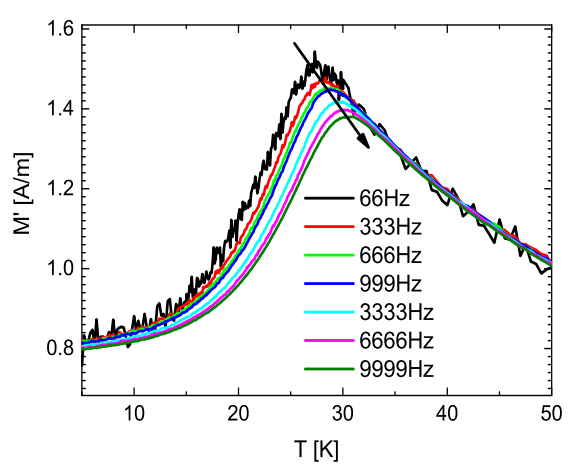

(c) $\mathrm{FeSr}_{2} \mathrm{Y}_{1.1} \mathrm{Ce}_{0.9} \mathrm{Cu}_{2} \mathrm{O}_{10-\mathrm{y}}$

Figure 8.3: Temperature dependence of the real part of the AC magnetization for seven different frequencies between $66 \mathrm{~Hz}$ to $10000 \mathrm{~Hz}$.

Such a spin-glass transition can be described by the relaxation time $\tau\left(T_{\mathrm{f}}\right)$ as described in section 2.3.3 with the Vogel-Fulcher-Equation [79],

$$
\tau\left(T_{\mathrm{f}}\right)=\tau_{0} \exp \left[\frac{E_{\mathrm{a}}}{k_{\mathrm{B}}\left(T_{\mathrm{f}}-T_{1}\right)}\right]
$$

where the freezing temperature $T_{\mathrm{f}}$ is the temperature where $M^{\prime}$ peaks. $E_{\mathrm{a}}$ is the activation energy, $k_{\mathrm{B}}$ is Boltzmann's constant and $T_{1}$ is a phenomenological parameter of the Vogel-Fulcher law for spin-glasses [80]. The measured data shows good agreement with such a fit as shown in figure 8.4 . 


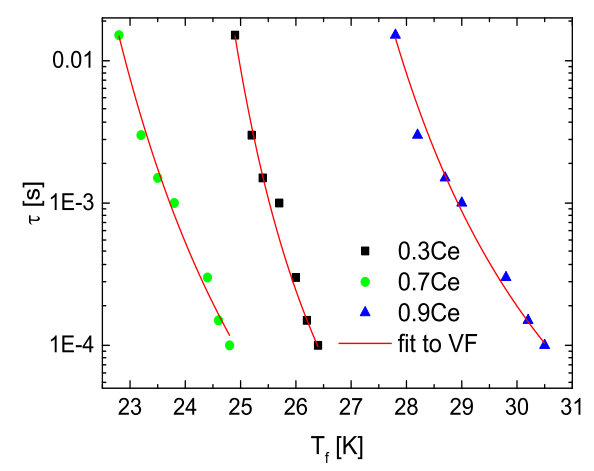

Figure 8.4: Relaxation times for different freezing temperatures with the fits to equation 2.6 .

Oxygen vacancies in the $\mathrm{FeO}_{2-\mathrm{y}}$ layers can cause highly disordered $\mathrm{Fe}-\mathrm{O}-\mathrm{Fe}$ superexchange interactions, which can lead to spin-glass behavior. Another option that would cause a spin-glass is site disorder with a significant fraction of $\mathrm{Fe}$ on the $\mathrm{Cu}$ sites and vice versa. 


\subsection{Susceptibility}

High field susceptibility measurements were performed for different Ce dopings ranging from $\mathrm{x}=0.2$ to $\mathrm{x}=0.9$ and varying oxygen content (an example for an oxygen saturated (OS) sample with $\mathrm{x}=0.7$ is shown in figure 8.5a). The Curie-Weiss (CW) temperature and the effective moment per formula unit can be obtained from these measurements as described in section 3.3 .2 and they are shown in figure $8.5 \mathrm{~b}$ and $8.5 \mathrm{c}$.

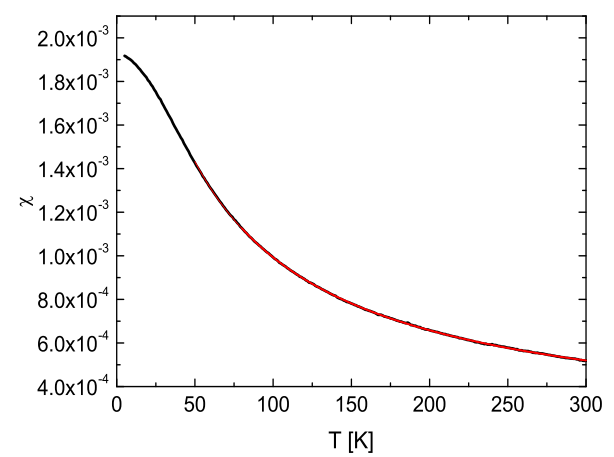

(a) Temperature dependence of the susceptibility for $\mathrm{x}=0.7$ in an oxygen saturated (OS) sample.

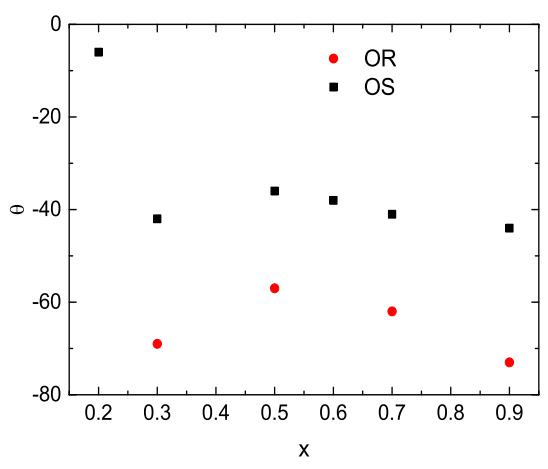

(b) Doping dependence of the CW temperature.

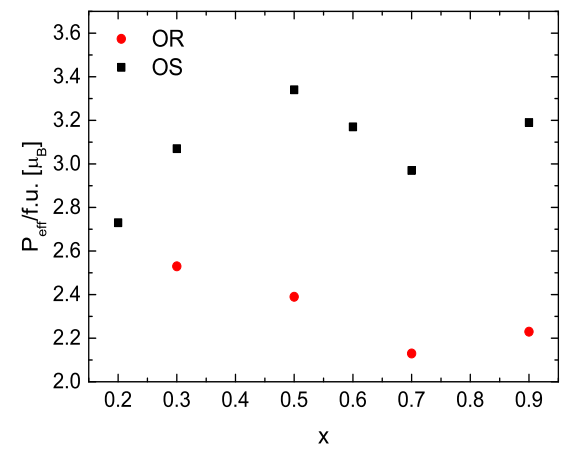

(c) Doping dependence of the effective moment.

Figure 8.5: An example of the temperature dependent susceptibility for $\mathrm{x}=0.7$ (a) and the doping dependence of the CW temperature (b) and the effective moment (c). 
The CW temperature, $\Theta$, is found to be at around $-40 \mathrm{~K}$ for the oxygen saturated samples, which suggests there are antiferromagnetic exchange interactions. The absolute value of the $\Theta$ is larger than those reported in previous studies [223, 240]. The oxygen reduced samples show an even higher negative value for the $\mathrm{CW}$ temperature, which is an indication of enhanced antiferromagnetic interactions in these samples. The temperature independent part of the susceptibility does not change much with doping and is in the range of $\chi_{0}=5 \times 10^{-5}$.

The effective moment per formula unit is only weakly dependent on the Ce content and the highest value of $P_{\text {eff,f.u. }}=3.33 \mu_{\mathrm{B}}$ is observed for $\mathrm{x}=0.5$. When there are 2 copper atoms and one iron atom per formula unit contributing to the effective moment per formula unit, it can then be written as:

$$
P_{\text {eff,f.u. }}^{2}=3 P_{\text {eff,ion }}^{2}=P_{\text {eff,Fe }}^{2}+2 * P_{\text {eff,Cu }}^{2}
$$

Pissas et al. [240] measured an effective moment per Ion of $P_{\text {eff,ion }}=1.9 \mu_{\mathrm{B}}$, which yields $P_{\text {eff,f.u. }}=3.29 \mu_{B}$ from equation 8.2 . Felner et al. 223] measured values of $P_{\text {eff,Fe }}=3.85 \mu_{B}$ for Fe1222 with Y and different rare-earth ions. They assumed that $P_{\text {eff,f.u. }}=P_{\text {eff,Fe }}$ and these values are consistent with neutron diffraction measurements. All of those measurements showed similar values within experimental uncertainties and these values are also similar to the observed effective moment in Fe1212 (previous chapter). However, there is a significant reduction in the observed $P_{\text {eff,fu. }}$ with the observed antiferromagnetic ordering (section 8.7), which is interpreted as an ordering of the $\mathrm{Cu}$ moments. The observed value of $P_{\text {eff,f.u. }}=3.33 \mu_{\mathrm{B}}$ results in $P_{\text {eff,ion }}=$ $1.92 \mu_{\mathrm{B}}$ and $P_{\text {eff,Fe }}=2.25 \mu_{\mathrm{B}}$ when $P_{\text {eff,Cu }}=1.73 \mu_{\mathrm{B}}$ is assumed. Similar to the compound Fe1212 there exist at least two different spin configurations of Fe in Fe1222. Their possible values are already shown in table 7.1, and assuming the same distribution of high spin $\left(S=5 / 2 \rightarrow 5.92 \mu_{\mathrm{B}}\right)$ and low spin $\left(S=1 / 2 \rightarrow 1.73 \mu_{\mathrm{B}}\right)$ configurations leads to a distribution of $96 \%$ Fe in the low spin state and only $4 \%$ in the high spin state. 


\subsection{Resistivity}

The resistivity, $\rho$, is shown in figure 8.6 and it strongly increases with decreasing temperature for all samples, which is likely to be due to variablerange-hopping (VRH). In this case the conductivity can be modeled with the Mott conductivity as described by equation 2.8, where the exponent $p=1 / 4$ for a Mott three-dimensional (3D) VRH, $p=1 / 3$ for a Mott two-dimensional (2D) VRH, and $p=1 / 2$ for Efros-Shklovskii (ES) VRH in the presence of Coulomb interactions [84, 86]. The observed data was subsequently fitted to equation 2.8 with different VRH exponents $p$, and the results of these fits to $2 \mathrm{D} V R H$ are shown for a Ce doping of $\mathrm{x}=0.3$ in figure $8.6 \mathrm{a}, \mathrm{x}=0.7$ in figure $8.6 \mathrm{c}$ and for $\mathrm{x}=0.9$ in figure 8.6e. Fits to $3 \mathrm{D} \mathrm{VRH}$ are shown in figure $8.6 \mathrm{~b}$, $8.6 \mathrm{~d}$ and $8.6 \mathrm{f}$, respectively. The data can be fitted over the whole temperature range for $\mathrm{x}=0.7 \mathrm{Ce}$ doping to $2 \mathrm{D} \mathrm{VRH}$, while a fit to $3 \mathrm{D} \mathrm{VRH}$ is only possible above $4 \mathrm{~K}$. In the case of $\mathrm{x}=0.3$ and $\mathrm{x}=0.9$ is it possible to fit to a $2 \mathrm{D}$ VRH as well as $3 \mathrm{D}$ VRH above $10 \mathrm{~K}$. Thus it seems that the data is the more consistent with 2D VRH, but the average hopping distance can be estimated to be in the order of several unit cells. There is also no anisotropic magnetoresistance observed as shown below. Hence, it makes more sense that it is $3 \mathrm{D} \mathrm{VRH}$ where a departure occurs at low temperatures (below $10 \mathrm{~K}$ for $\mathrm{x}=0.3$ and 0.9 and below $5 \mathrm{~K}$ for $\mathrm{x}=0.7$ ). No other report is known, which discusses the resistivity of Fe1222 and hence it is not possible to determine where the localized carriers reside. It is possible that the localized carriers reside at or near the oxygen vacancies because the resistivity was found to significantly increases as the oxygen content is reduced. Another possibility would be that the $\mathrm{Fe}$ on the $\mathrm{Cu}(2)$ site creates enough disorder so that localized states occur, leading to the observed behavior. 


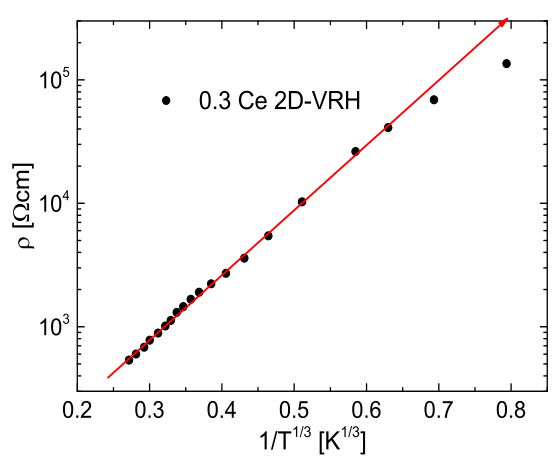

(a) $\mathrm{x}=0.3$ for $2 \mathrm{D}-\mathrm{VRH}$.

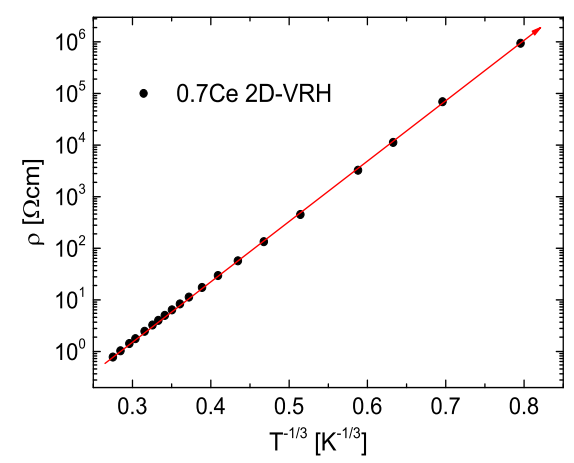

(c) $\mathrm{x}=0.7$ for $2 \mathrm{D}-\mathrm{VRH}$.

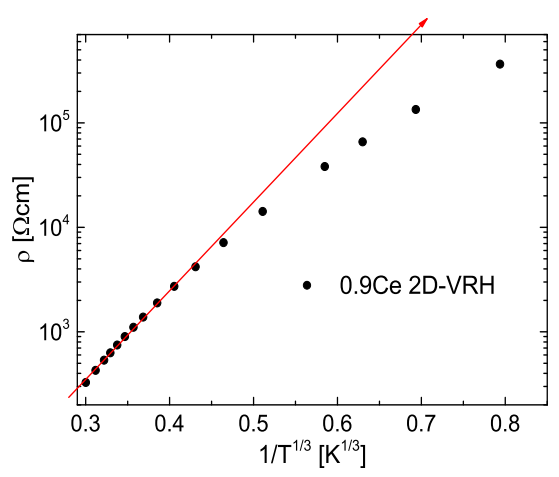

(e) $\mathrm{x}=0.9$ for $2 \mathrm{D}-\mathrm{VRH}$.

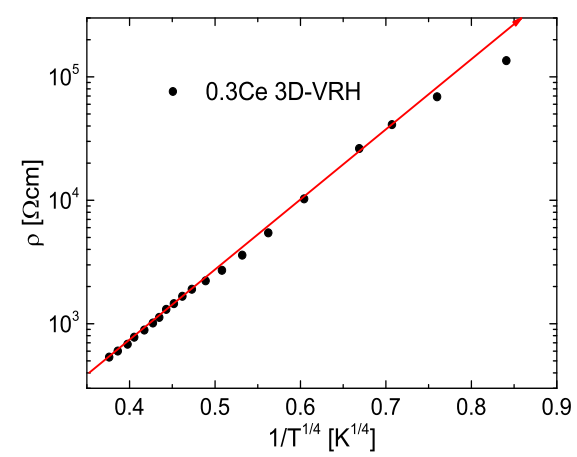

(b) $\mathrm{x}=0.3$ for $3 \mathrm{D}-\mathrm{VRH}$.

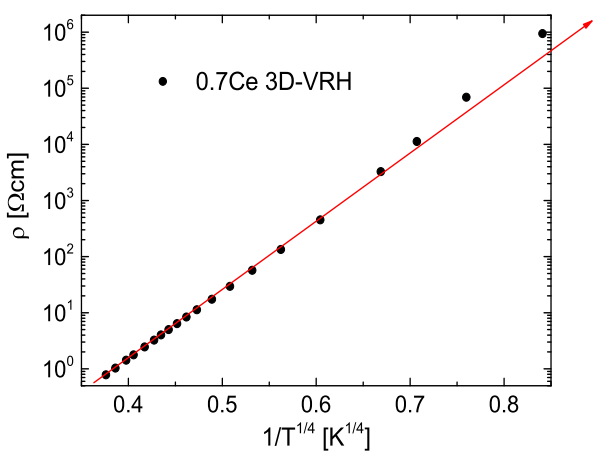

(d) $\mathrm{x}=0.7$ for $3 \mathrm{D}-\mathrm{VRH}$.

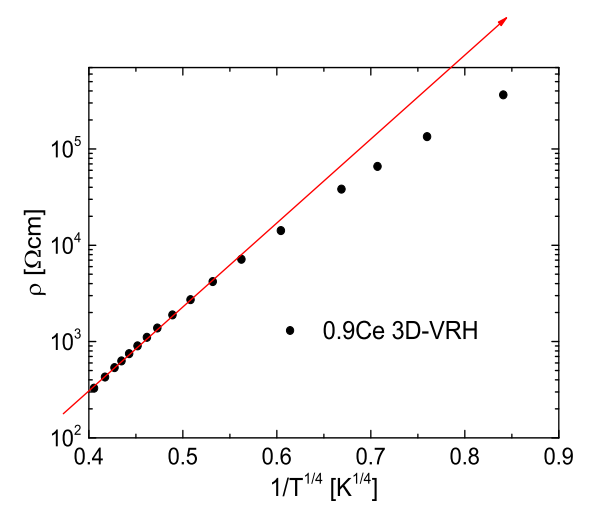

(f) $\mathrm{x}=0.9$ for $3 \mathrm{D}-\mathrm{VRH}$.

Figure 8.6: Resistivity against a temperature dependence of $T^{-1 / 3}(2 \mathrm{D})$ and $T^{-1 / 4}$ (3D) for different Ce content. 


\subsection{Magnetoresistance}

The magnetoresistance (MR) was introduced in section 3.3.1 and was defined in equation 2.11. Figure 8.7 shows the field dependence of the MR for all samples, which is negative over the whole temperature range and can reach values up to $-22 \%$ for $\mathrm{x}=0.7$. An additional positive component is observed for temperatures of $4 \mathrm{~K}$ and below for all samples, which is most visible at $2 \mathrm{~K}$. This can be seen more clearly in figure 8.8 where the MR is plotted for a field of a) $3 \mathrm{~T}$ and b) $8 \mathrm{~T}$. It is apparent that there is a positive contribution to the MR at least up to $4 \mathrm{~K}$. It may be that the positive MR at low field (see figure 8.8 and 8.7 ) and for temperatures below $5 \mathrm{~K}$ occurs because the Nguen, Spivak, and Shklovskii (NSS) [97, 107] theory of negative VRH quantum interference MR assumes scattering between static or frozen spins for each hop. However, it has been noted that it is important to also include scattering from a fraction of free spins that are localized at a scattering site and where there is scattering from them for each hopping event [249, 250]. This can lead to a positive MR that is only observed or reported at low temperatures [249, 250] as seen in our sample (figure 8.8 and 8.7). 


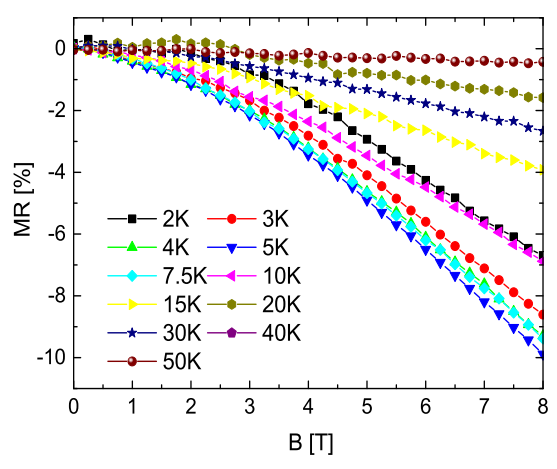

(a) $\mathrm{x}=0.3$

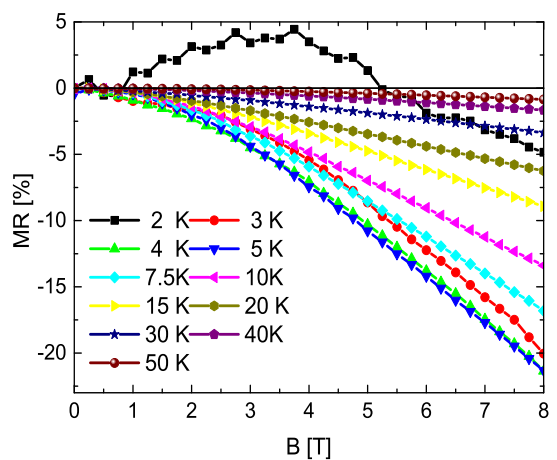

(b) $\mathrm{x}=0.7$

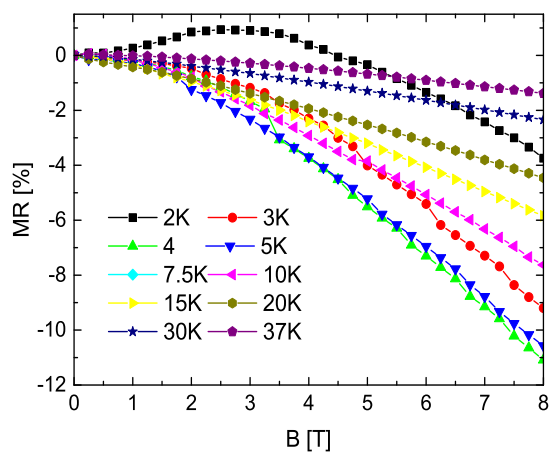

(c) $\mathrm{x}=0.9$

Figure 8.7: Magnetic field dependence of the MR for different Ce doping. 


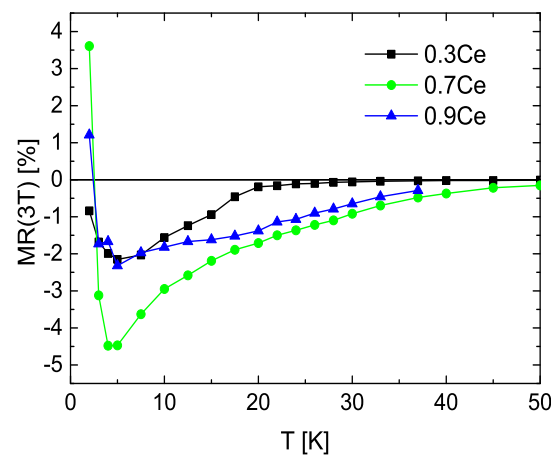

(a) $3 \mathrm{~T}$

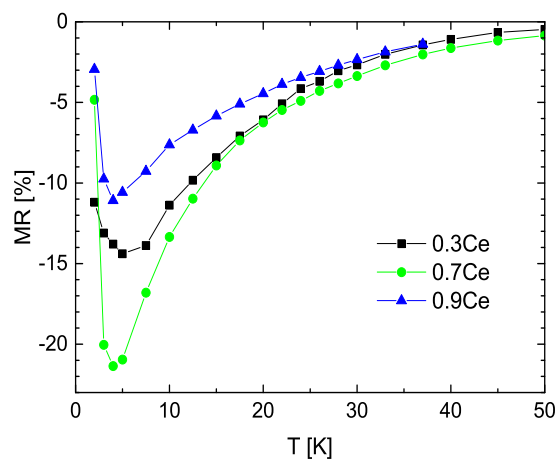

(b) $8 \mathrm{~T}$

Figure 8.8: Temperature dependence of the MR at different magnetic fields and doping.

The magnitude of the normalized $\mathrm{MR}$ at and above $5 \mathrm{~K}$ is plotted next in figure 8.9, where the MR was normalized to the MR at $8 \mathrm{~T}$. It can be seen that the magnetic field dependence of the MR is nearly the same for temperatures below $15 \mathrm{~K}$ and at $40 \mathrm{~K}$, which suggests a common underlying mechanism. There is a small difference for the temperature range between $15 \mathrm{~K}$ to $30 \mathrm{~K}$. This temperature range also includes the spin-glass temperature $(23 \mathrm{~K})$ and hence the small deviation may somehow be related to this spin-glass transition. 


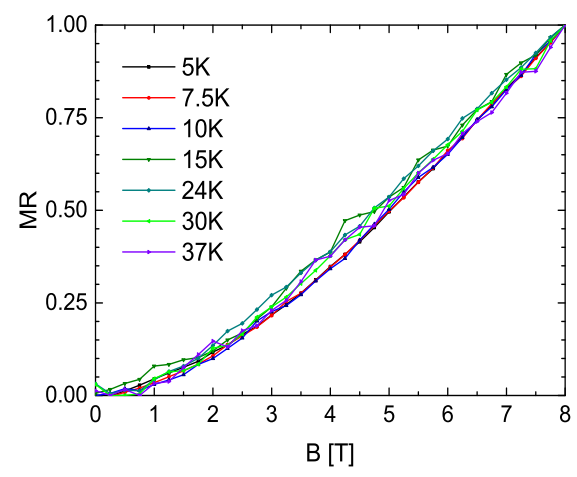

(a) $\mathrm{x}=0.3$

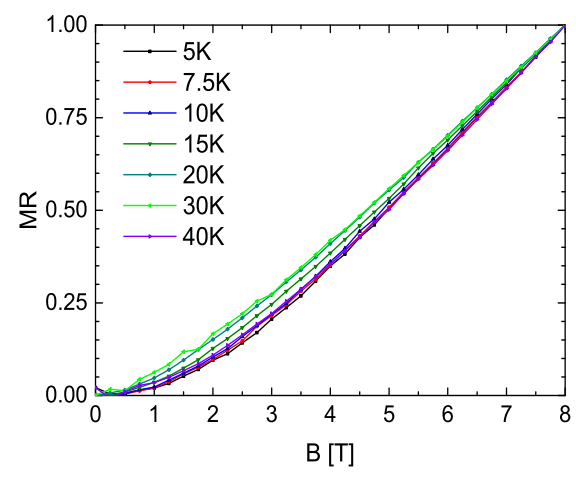

(b) $\mathrm{x}=0.7$

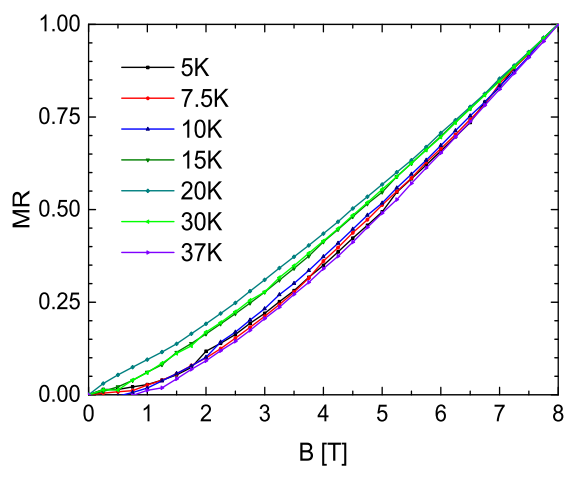

(c) $\mathrm{x}=0.9$

Figure 8.9: Magnetic field dependence of normalized MR for different temperatures going through the spin-glass transition.

Large MR values have previously been reported in other compounds displaying 2D and 3D VRH [99, 249 259]. VRH is a competition between tunneling to nearby states and thermally activated hopping to more distant states [84]. The resultant hopping rate between two states can be written as:

$$
\frac{1}{\tau_{\mathrm{h}}}=\frac{1}{\tau_{\mathrm{h}, 0}} \exp \left(-\frac{2 D}{\xi}-\frac{W}{k_{\mathrm{B}} T}\right)
$$

where $D$ is the distance between the two localized states, $\xi$ is the localization radius, $W$ is the energy difference between the two localized states, and $1 / \tau_{\mathrm{h}, 0}$ is a constant that includes the attempt frequency. The hopping rate 
can change if $\xi$ is affected by the applied magnetic field. This can occur where the applied magnetic field can lead to a decrease in $\xi$ and hence a shrinkage of the localized electron wavefunction [98, 255, 256]. It results in a decrease in the hopping rate and an increase in the resistance that leads to a positive MR. This wavefunction shrinkage model cannot explain our data because we observe negative MR values over most of the magnetic field and temperature ranges. It is likely that the MR is due to VRH quantum interference.

As stated in the theory section 2.5.1 VRH QI can lead to positive or negative MR. A negative VRH quantum interference MR can depend on the angle between the sample and the applied magnetic field for thin samples [250, 260]. However, if many hops dominate the conductivity then there is no MR anisotropy [260]. This is observed experimentally in bulk samples [251, 253 255, 257 259, 261]. It also appears to be the case for our sample as can be seen in figure 8.10 where the MR is plotted at $5 \mathrm{~K}$ with the applied magnetic field parallel and perpendicular to the sample.

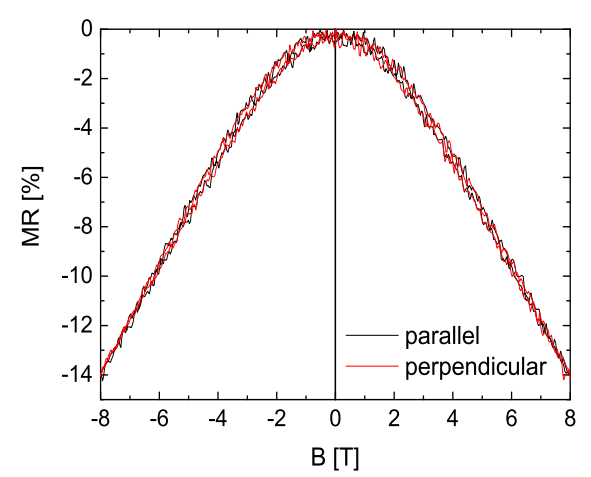

Figure 8.10: Magnetic field dependence of the MR at $5 \mathrm{~K}$ for parallel (black) and perpendicular (red) to the external magnetic field.

It is apparent that there is a negligible MR anisotropy. This is surprising given the good fit to the resistivity data to $2 \mathrm{D} \mathrm{VRH}$ (figure 8.6c) and where an anisotropy in the MR might be expected. As already mentioned above, it is more likely that the underlying conduction mechanism is $3 \mathrm{D} \mathrm{VRH}$ and there is a departure from the expected temperature dependence at and below $4 \mathrm{~K}$ (figure 8.6c). The onset temperature of this departure also corresponds with 
the temperature where there is a change in the magnetic field dependence of the MR (see figure 8.7 and 8.8) and hence there may be a change in the hopping mechanism. However, this is speculative and requires theoretical input and further research outside the scope of this present study. For low field the MR is proportional to $B^{2}$ [253, 260] and it becomes linear for higher field [253]. This can be seen in figure 8.11 where the MR is plotted against $B^{2}$ at $10 \mathrm{~K}$ and $20 \mathrm{~K}$. The $\mathrm{MR}$ is found to be proportional to $B^{2}$ below $\sim 2.7 \mathrm{~T}$ at $10 \mathrm{~K}$ and below $\sim 1.5 \mathrm{~T}$ at $20 \mathrm{~K}$. The MR follows a linear field dependence for $B>4 \mathrm{~T}$ at both temperatures (figure 8.12). Similar quadratic and linear magnetic field regions are found after doing the same analysis on the data in figure 8.9 and for temperatures from 5 to $50 \mathrm{~K}$ for all samples. 


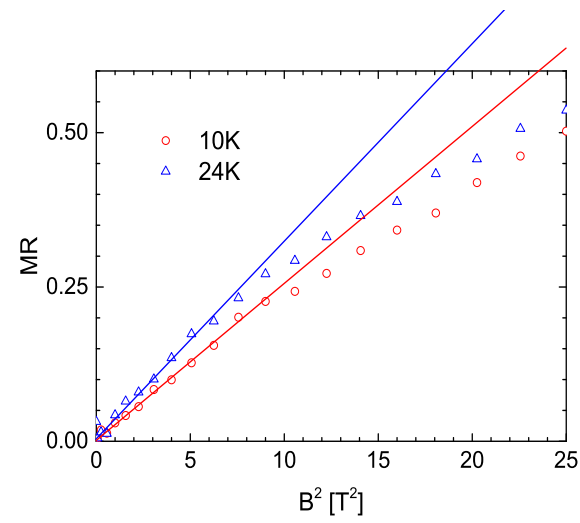

(a) $\mathrm{x}=0.3$

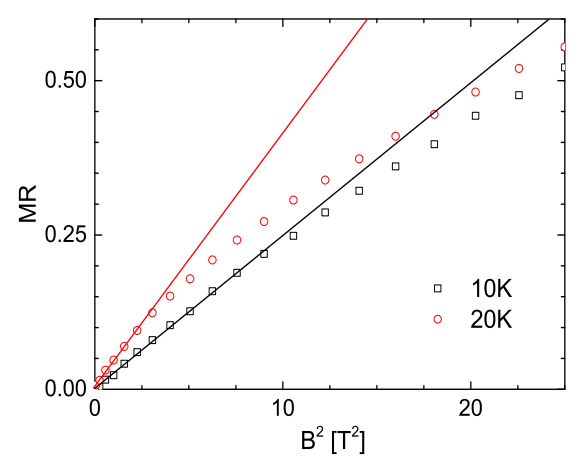

(b) $\mathrm{x}=0.7$

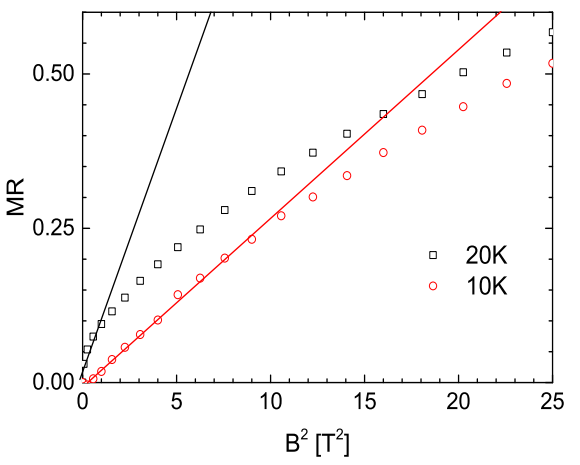

(c) $\mathrm{x}=0.9$

Figure 8.11: The MR for 10 and $20 \mathrm{~K}$ for the quadratic field dependence in the low field region. 


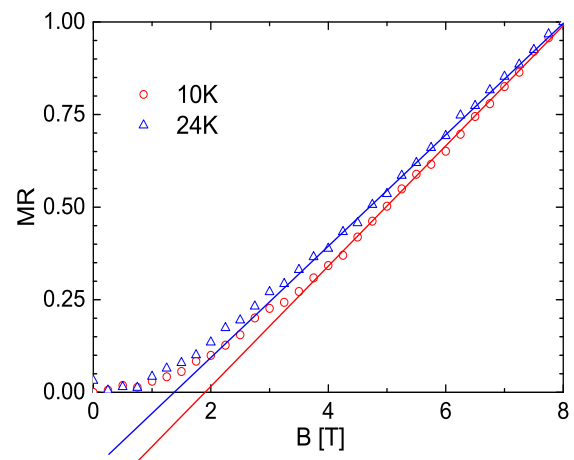

(a) $\mathrm{x}=0.3$

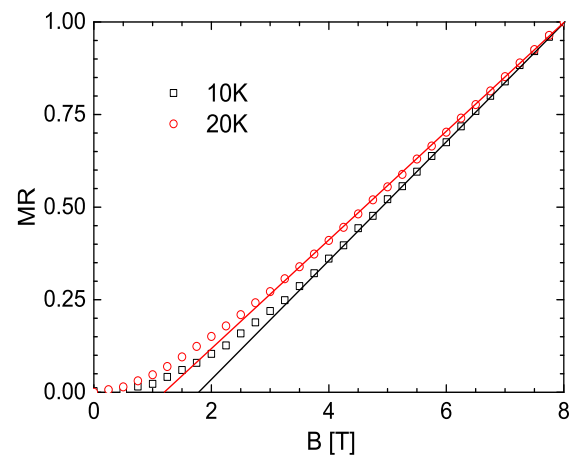

(b) $\mathrm{x}=0.7$

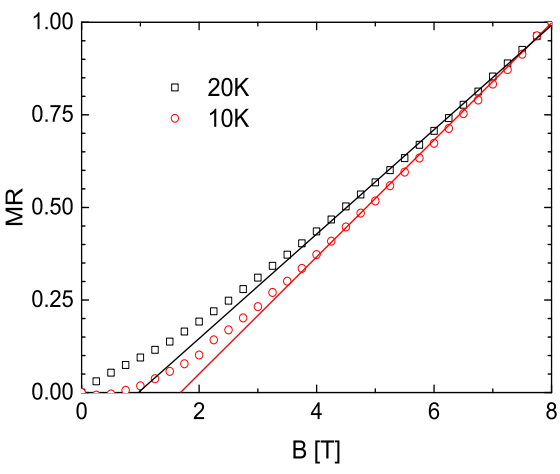

(c) $\mathrm{x}=0.9$

Figure 8.12: The MR for 10 and $20 \mathrm{~K}$ for the linear field dependence in the high field region. 


\subsection{The effect of oxygen loading}

This section will discuss the effects of different oxygen content through a comparison between oxygen reduced (OR) (8.2) and oxygen saturated (OS) samples. The masses of these samples were measured before and after oxygen loading, which allowed the oxygen difference to be estimated as $\Delta y=$ 1.20(0.05). For HTSC cuprates there exists a correlation between the room temperature thermopower and the hole concentration [245], hence thermopower values were measured for all samples in the OR and OS state. The results are shown in figure 8.13 .

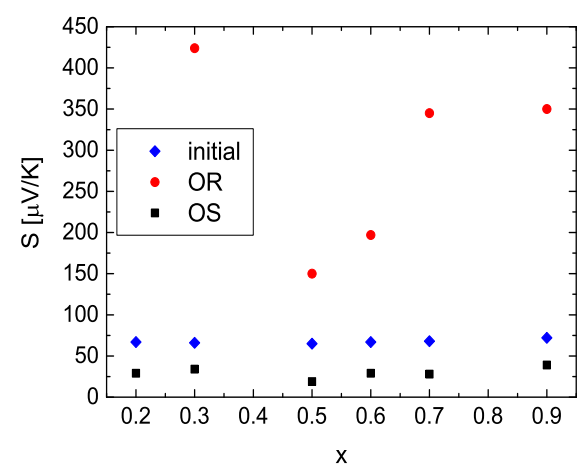

Figure 8.13: Thermopower at room temperature for OR and OS samples of Fe1222.

The initial thermopower values are around $\sim 70 \mu \mathrm{VK}^{-1}$ and a huge increase was observed after processing in Ar, towards values ranging from $150 \mathrm{\mu V} \mathrm{K}^{-1}$ to $424 \mathrm{\mu V} \mathrm{K}^{-1}$, which is a good indication of oxygen removal. After the final step where additional oxygen was introduced even lower values of $S(295 K)$ are observed, which range from $19 \mu \mathrm{V} \mathrm{K}^{-1}$ to $39 \mu \mathrm{V} \mathrm{K}^{-1}$. These thermopower values [245] correspond to the number of doped holes per $\mathrm{Cu}$, $p$, being 0.11 to 0.09 for OS and 0.03 to 0.00 for the OR sample. $p$ for the OS sample is in the range where superconductivity is expected $(p>0.05)$. The absence of superconductivity is most likely due to pair breaking by a small amount of $\mathrm{Fe}$ on the $\mathrm{Cu}$ site in the $\mathrm{CuO}_{2}$ plane. For the HTSC cuprate $\mathrm{YBa}_{2} \mathrm{Cu}_{3} \mathrm{O}_{6+\mathrm{y}}$ less than $5 \% \mathrm{Fe}$ is required to reduce the superconducting 
transition temperature to zero [262]. The hole concentration in the OR sample is consistent with a non-superconducting and insulating sample where the $\mathrm{Cu}$ moments in the $\mathrm{CuO}_{2}$ plane order antiferromagnetically. The ZFC and FC magnetization data from the OS samples (not shown) are similar to the magnetization shown in figure 8.2 (Sambale et al. [13]) where there is a peak in the ZFC moment at $\sim 22 \mathrm{~K}$ that arises from a spin-glass [13]. It can be seen in figure 8.14 that the peak occurs at approximately the same temperature in the OR sample and hence it is apparent that the spin-glass temperature is not affected by a large reduction in the oxygen content.

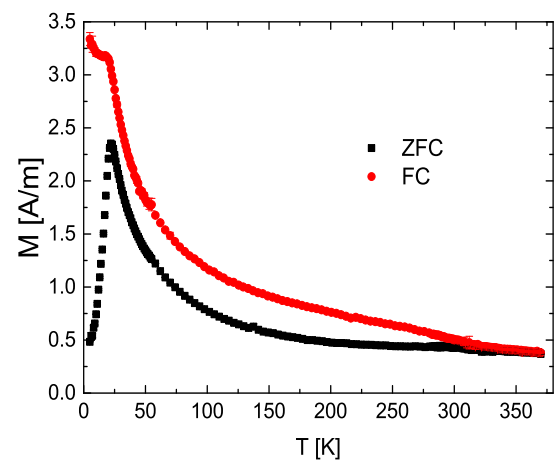

Figure 8.14: ZFC and FC $(2 \mathrm{mT})$ magnetization for the oxygen reduced (OR) Fe1222 with 0.5 Ce doping.

The magnetization for the OR sample also has a broad peak at $\sim 300 \mathrm{~K}$ and there is significant irreversibility below this temperature. This can be interpreted in terms of antiferromagnetic order with a Nèel temperature around $300 \mathrm{~K}$. A similar ordering is observed in the insulating phase of the cuprates, where the ordering temperature ranges from $320 \mathrm{~K}$ to $415 \mathrm{~K}$ [263, 264]. In these compounds the antiferromagnetic ordering is assigned to an ordering of the $\mathrm{Cu}$ spins in the $\mathrm{Cu}(2)$ site and it makes sense to assume the same happens in our OR samples.

The susceptibility of the OR and OS samples were determined through high field measurements and an example is shown in figure 8.15 for $\mathrm{x}=0.5$. They were fitted to a CW temperature dependence and the results incorporated in figure $8.5 \mathrm{~b}$, where it is shown that the $\mathrm{CW}$ temperature of the 
OR samples is lower. This is an indication of enhanced antiferromagnetic interactions between the Fe moments in the OR samples.

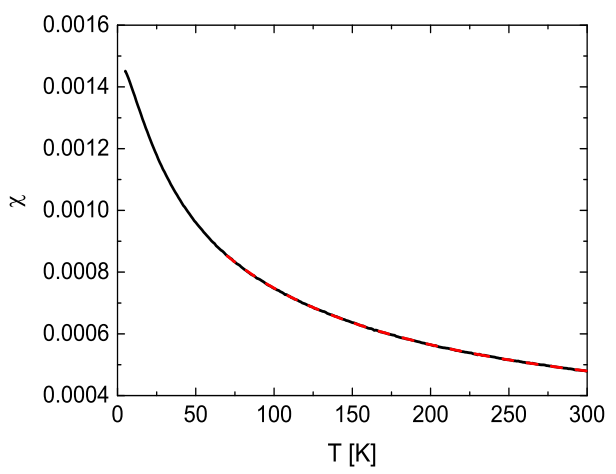

(a) $\chi-$ OR

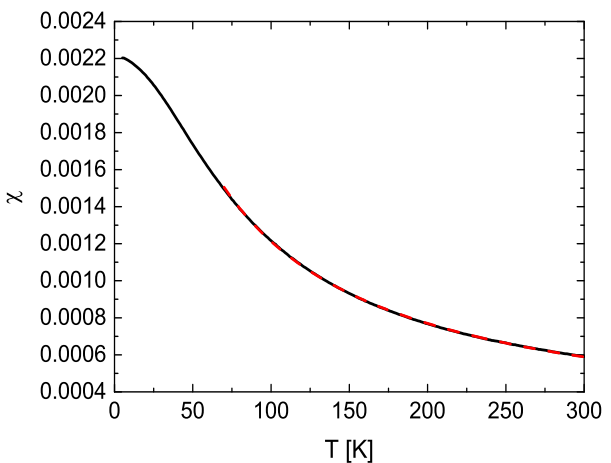

(c) $\chi-\mathrm{OS}$

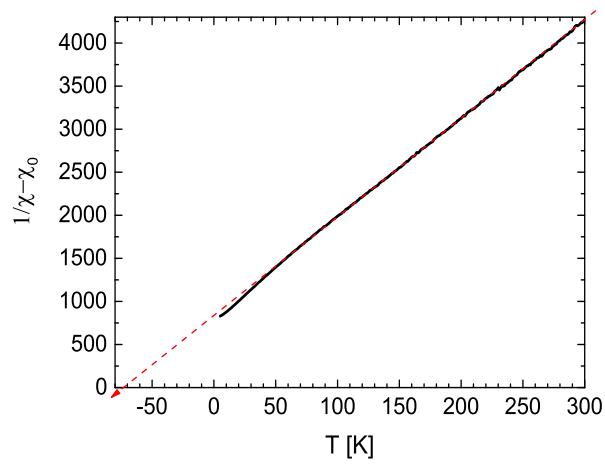

(b) $1 /\left(\chi-\chi_{0}\right)-$ OR

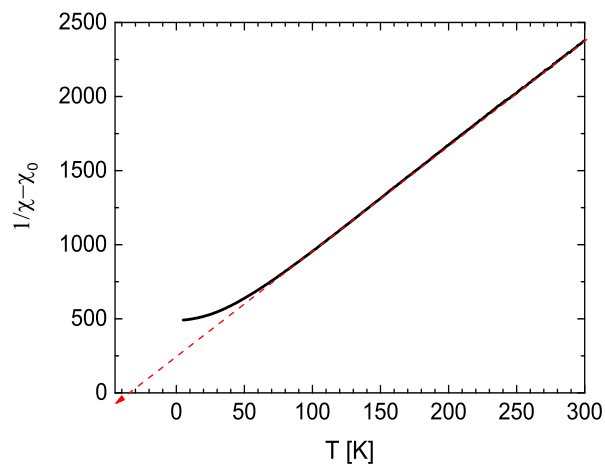

(d) $1 /\left(\chi-\chi_{0}\right)-$ OS

Figure 8.15: Temperature dependence of the susceptibility and $1 /\left(\chi-\chi_{0}\right)$ of the oxygen reduced (OR) and the oxygen saturated (OS) samples of Fe1222 with $\mathrm{x}=0.5$.

The $P_{\text {eff }}^{\text {f.u. }}$ of the OR samples are also different as is shown in figure $8.5 \mathrm{c}$ where they are significantly smaller than those of the OS samples. The $\mathrm{Cu}$ is ordered in the investigated temperature region and hence there is no observable static moment in comparison to the OS samples. Therefore, copper has no contribution to the observed effective moment in the OR sample. Calculating the effective moment per Fe yields a value around $P_{\text {eff }}^{\mathrm{Fe}}=2.63 \mu_{\mathrm{B}}$, which is higher than the value observed for the OS samples. This change in 
$P_{\text {eff }}^{\mathrm{Fe}}$ also suggests a different Fe distribution where $12 \% \mathrm{Fe}$ are in the high spin configuration and $88 \%$ in the low spin configuration.

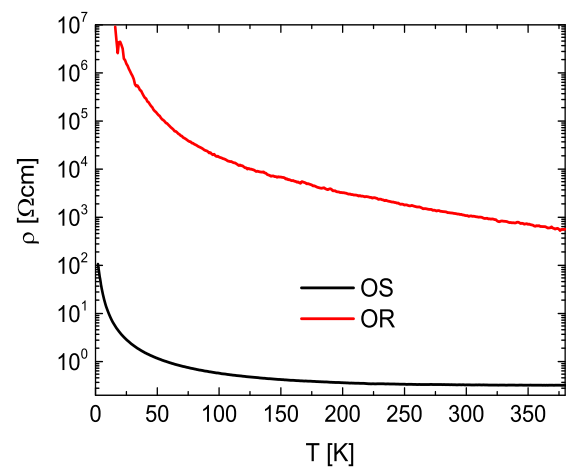

(a) Resistivity

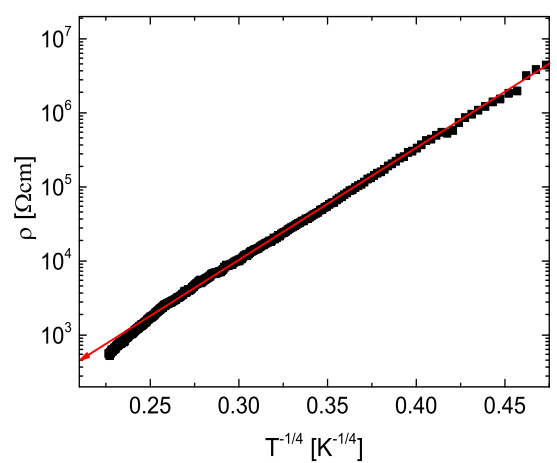

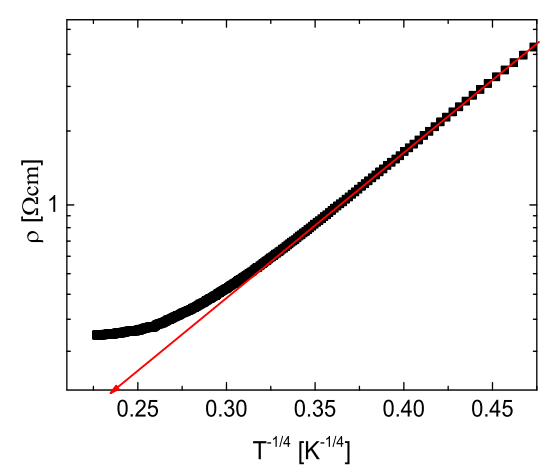

(b) Low temperature resistivity of OS with fit to $3 \mathrm{D}-\mathrm{VRH}$.

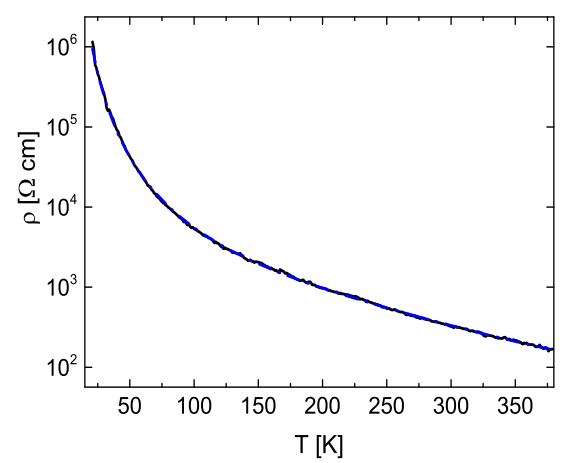

(c) Low temperature resistivity of OR with fit (d) Low temperature resistivity of OR sample to $3 \mathrm{D}-\mathrm{VRH}$. with fit to equation 8.5 .

Figure 8.16: Temperature dependence of the resistivity for OR and OS samples of Fe1222 with $\mathrm{x}=0.5$.

The results from resistivity measurements on the OS and OR samples of $\mathrm{FeSr}_{2} \mathrm{Y}_{1.5} \mathrm{Ce}_{0.5} \mathrm{Cu}_{2} \mathrm{O}_{10-\mathrm{y}}$ are shown in figure 8.16. The resistivity from the OR sample is $100 \Omega \mathrm{cm}$ at $370 \mathrm{~K}$ and it increases with decreasing temperature. Figure 8.16 also shows that the resistivity below $\sim 260 \mathrm{~K}$ can be fitted to $\mathrm{VRH}$ resistivity [84 86] from equation 2.8. 


$$
\rho=\rho_{0} \exp \left[\frac{C_{0} \alpha^{3}}{N\left(E_{\mathrm{F}}\right) k_{\mathrm{B}} T}\right]^{1 / 4}
$$

where $C_{0}$ is a constant, $\alpha$ is the inverse localization length, $k_{\mathrm{B}}$ is Boltzmann's constant and $N\left(E_{\mathrm{F}}\right)$ is the density of localized states at the Fermi energy. Thus, a lower $N\left(E_{\mathrm{F}}\right)$ leads to a higher resistivity. It is shown in figure $8.16 \mathrm{c}$ in a plot of the resistivity against $1 / T^{1 / 4}$ that the data can be modeled by equation 8.6 only for temperatures below $195 \mathrm{~K}$. A fit of the resistivity data to Mott 2D variable range hoping (equation 2.8 with $1 / 3$ ) and variable-rangehopping in the presence of Coulomb interactions (equation 2.8 with $1 / 2$ ) is also not possible over the full temperature range. However, there might be more than one conductivity channel for the OR samples and it is possible that there is variable-range-hopping between localized states near the Fermi level as well as activated band edge conduction. Band edge conduction could occur across a small bandgap in the $\mathrm{CuO}_{2}$ plane and there could be variablerange-hopping between localized states in the oxygen deficient $\mathrm{FeO}_{2-\mathrm{y}}$ plane. There are more possibilities than that, for example that there is two channel conduction via localized states at the Fermi level and activation band edge conduction in the $\mathrm{CuO}_{2}$ plane and where the localized states could arise from some $\mathrm{Fe}$ on the $\mathrm{Cu}$ sites in the $\mathrm{CuO}_{2}$ plane. We note that the room temperature thermopower indicates that $p$ is $\sim 0.03$ which is the hole doping range where insulating or semiconducting behavior in the $\mathrm{CuO}_{2}$ plane is observed in the superconducting cuprates. Thus, we model the OR resistivity as,

$$
\rho(T)=\frac{1}{\rho_{0 \exp \left(T_{0} / T\right)^{1 / 4}+\rho_{1}\left[\exp \left(E_{\mathrm{g}} / k_{\mathrm{B}} T\right)^{-1}\right]}}
$$

where $\rho_{1}$ is the temperature independent activated band edge conduction resistivity prefactor, and $E_{\mathrm{g}}$ is the bandgap energy. Figure $8.16 \mathrm{~d}$ shows that the $\mathrm{OR}$ resistivity data can be fitted to equation 8.5 , where $T_{0}=1.34 \times 10^{6} \mathrm{~K}$ and $E_{\mathrm{g}}=160(7) \mathrm{meV}$. The absence of superconductivity is most likely due to pair breaking by a small amount of $\mathrm{Fe}$ on the $\mathrm{Cu}$ site in the $\mathrm{CuO}_{2}$ plane. We note that less than $5 \% \mathrm{Fe}$ is required to reduce the superconducting transition temperature to zero [262]. 
The estimated amount of holes per copper, $p$, is even higher than that for an oxygen loaded $\mathrm{x}=0.7$ sample that did not have the additional $700{ }^{\circ} \mathrm{C}$ argon annealing step. Furthermore, unlike the oxygen loaded samples mentioned previously, the resistivity cannot be modeled in terms of variablerange-hopping (figure 8.16b). This is not surprising because the room temperature resistivity of $44 \mathrm{~m} \Omega \mathrm{cm}$ is too low for variable-range-hopping. It also cannot be fitted to the activated band edge conduction mentioned above. While the estimated $p$ of 0.11 suggests that the OS sample should be similar to an underdoped $(p<0.16)$ superconducting cuprate, the temperature dependence of the resistivity is clearly different. In particular, the resistivity is too high and a large increase in the resistivity is not consistent with a normal-state pseudogap.

It is also unlikely to be due to the Kondo effect characterized by a $\ln (T)$ divergence below the Kondo temperature from scattering by magnetic ions (e.g. Fe moments in the $\mathrm{CuO}_{2}$ plane), because there is no upturn in the resistivity even at $370 \mathrm{~K}$. This would imply a Kondo temperature above $370 \mathrm{~K}$, which does not seem to be realistic. The large increase in the resistivity by more than two orders of magnitude as the temperature is reduced is also too much to be consistent with a Kondo effect.

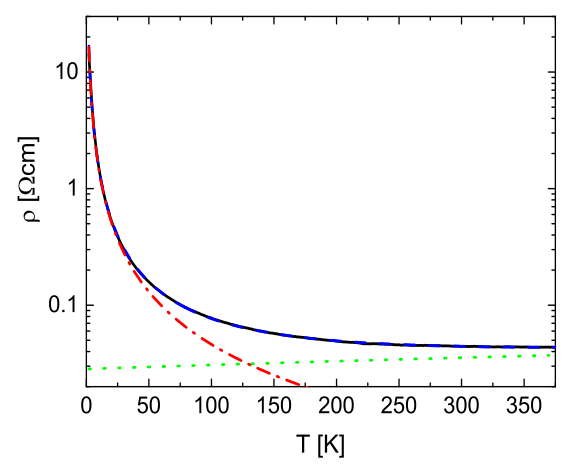

Figure 8.17: Plot of the resistivity against temperature for an oxygen saturated sample (solid curve). The dashed curve is a fit to equation 8.6.

Also shown are the metallic (doted curve) and the $T^{-3 / 2}$ (dot-dash) contribution to the fit. 
It is shown in figure 8.17 that a reasonable fit can be achieved, when the OS resistivity data is fitted to the following equation:

$$
\rho(T)=\left[\rho_{0}+a T\right]+\frac{b}{T^{3 / 2}}
$$

where $\rho_{0}$ is the temperature independent resistivity, and $a, b$ are temperature independent constants. The first term is a metallic linear resistivity. However, a Fermi liquid $T^{2}$ metallic resistivity can also provide a reasonable fit to the data. The second $T^{-3 / 2}$ term accounts for the large increase in the resistivity as the temperature is reduced and such a temperature dependence can be observed when there is disorder and weak localization. Provided the disorder is not too large then the $3 \mathrm{D}$ resistivity at low temperatures and to lowest order can have an additional weak localization quantum interference contribution to the resistivity that can be written as [95, 96],

$$
\Delta \sigma=a_{1} T^{p / 2}+a_{2} T^{1 / 2}
$$

where $a_{1}$ and $a_{2}$ are constants. The first term has $p=2$ or 3 depending on whether the scattering rate is determined by electron-electron or electronphonon scattering. Thus, a $T^{-3 / 2}$ term could occur provided that the first term is significantly larger than the second term and $p=3$. However, the room temperature resistivities are too high $(>44 \mathrm{~m} \Omega \mathrm{cm})$ to make weak localization a likely explanation and the more than two orders of magnitude increase in the resistivity as the temperature is reduced is not seen in materials where weak localization quantum interference is observed. Thus, the $T^{-3 / 2}$ term is likely to arise from considerable site disorder in the $\mathrm{CuO}_{2}$ plane and the $\mathrm{FeO}_{2-\mathrm{y}}$ layer as well as oxygen vacancy disorder in the $\mathrm{FeO}_{2-\mathrm{y}}$ layer. 


\subsection{Summary and conclusions}

This chapter presented the electronic and magnetic measurements on polycrystalline $\mathrm{FeSr}_{2} \mathrm{Y}_{2-\mathrm{x}} \mathrm{Ce}_{\mathrm{x}} \mathrm{Cu}_{2} \mathrm{O}_{10-\mathrm{y}}$ samples. A spin-glass transition was found at $23 \mathrm{~K}$ and the freezing temperature is only weakly dependent on the Ce content. The spin-glass may occur in the oxygen deficient $\mathrm{FeO}_{2-\mathrm{y}}$ plane where there could be disordered Fe-O-Fe superexchange interactions, or it may be due to site disorder where there are significant fractions of $\mathrm{Fe}$ and $\mathrm{Cu}$ on both transition metal sites. The conduction mechanism is $3 \mathrm{D}$ variable-rangehopping (VRH) for all samples above $10 \mathrm{~K}$, with a departure thereafter. The magnetoresistance at low temperatures is as high as $-22 \%$ at $8 \mathrm{~T}$ for $\mathrm{x}=0.7$ Ce doped sample and shows a similar trend for all samples. The magnetoresistance is due to quantum interference effects that occur when there is hopping over a large number of scattering centers. The magnetoresistance for all samples at and above $5 \mathrm{~K}$ is negative and follows the magnetic field dependence expected for VRH quantum interference when there is scattering from static or frozen spins. A positive magnetoresistance for all samples occurs for temperatures below $5 \mathrm{~K}$ that may be due to a contribution from hops where there is scattering from localized free spins that is not accounted for in the Nguen, Spivak, and Shklovskii (NSS) model.

The effect of oxygen reduction was also studied on oxygen saturated (OS) and oxygen reduced (OR) $\mathrm{FeSr}_{2} \mathrm{Y}_{2-\mathrm{x}} \mathrm{Ce}_{\mathrm{x}} \mathrm{Cu}_{2} \mathrm{O}_{10-\mathrm{y}}$. The oxygen content has no significant effect on the spin-glass temperature. However, antiferromagnetic order is observed around $300 \mathrm{~K}$ in the OR samples, which is interpreted as an ordering of the $\mathrm{Cu}$ spins in the $\mathrm{CuO}_{2}$ plane. The Curie-Weiss temperature shows an increase in magnitude for the OR samples, which is a sign of stronger antiferromagnetic exchange interactions from Fe. There is also a decrease in the effective moment per formula unit for the OR samples, which is due to the fact that the $\mathrm{Cu}$ moments are ordered in the investigated temperature region and have no static magnetic moment there. Hence, the OR samples have a higher effective moment per Fe and thus a larger amount of $\mathrm{Fe}$ in the high spin configuration. The resistivity data from the oxygen reduced sample can be modeled in terms of variable-range-hopping and conduction 
across a small gap in a semiconductor with a bandgap of $160 \mathrm{meV}$. The resistivity from the oxygen saturated samples is more than three orders of magnitude lower. Unlike the resistivity measurements on the oxygen loaded samples in earlier studies, no evidence for variable-range-hopping is found. It may be that the additional high temperature argon annealing step in the present study has reduced the disorder in the $\mathrm{CuO}_{2}$ plane by reducing the fraction of $\mathrm{Fe}$ on the $\mathrm{Cu}$ site in the $\mathrm{CuO}_{2}$ plane. The resistivity of the OS samples can be modeled with a metallic resistivity and an additional term that is proportional to $T^{-3 / 2}$. The origin of this term is not clear but it does show that there is significant disorder. From the room temperature thermopower correlation observed in the HTSC's, it would be expected that $p \sim 0.11$ and the oxygen saturated sample should be superconducting. It is likely that there is sufficient $\mathrm{Fe}$ on the $\mathrm{CuO}_{2}$ sites that superconductivity is suppressed. 


\section{Chapter 9}

\section{Summary}

This thesis is about a study of iron-based superconductors that encompass the iron-pnictides and the iron-cuprate superconductors. It was found that disorder plays a major role in the resultant electronic and magnetic properties. Disorder can be seen in the NMR spectra of $\mathrm{CeFeAsO}_{1-\mathrm{x}} \mathrm{F}_{\mathrm{x}}$ in the form of charge disorder and it is apparent in $\mathrm{FeSr}_{2} \mathrm{Y}_{2-\mathrm{x}} \mathrm{Ce}_{\mathrm{x}} \mathrm{Cu}_{2} \mathrm{O}_{10-\mathrm{y}}$ where there is a spin glass and strong localization for certain doping levels. The two sections below provide a more detailed description of the compounds studied in this thesis. This is followed by a section on further work. 


\subsection{Iron-pnictide superconductor}

The iron-pnictide superconductors were studied via two chosen groups of compound. The first group is the $\mathrm{CeFeAsO}_{1-\mathrm{x}} \mathrm{F}_{\mathrm{x}}$ compound, where polycrystalline samples were studied. It was found that the resistivity for the non-superconducting sample $(x=0.07)$ follows a linear temperature dependence while the superconducting samples show a Fermi liquid temperature dependence above the superconducting transition temperature. Furthermore, fluctuation conductivity is observed when approaching the superconducting transition temperature with a crossover from 2D towards 3D fluctuation conductivity. This crossover is observed closer to superconducting transition temperature for the underdoped sample $(x=0.13)$ compared to the other superconducting samples, which is due to a smaller coherence length in the $x=0.13$ sample. The superconducting region of the resistivity was analyzed within the thermally activated flux-flow model and vortex motion could be observed within the model. The activation energy and the critical current density $J_{\mathrm{C}}$ are observed to peak around the optimal doping region. They are correlated which is accounted for in the two fluid-flux creep model. The irreversibility field can be interpreted in terms of 3D vortex lattice melting and the irreversibility field is higher for higher fluoride concentrations. An orbital limiting field, $B_{\mathrm{c} 2}^{\text {orb }}$, of up to $150 \mathrm{~T}$ for $x=0.13$ was calculated using the Werthamer-Helfand-Hohenberg formula and it was found that an orbital limiting effect alone is not enough (Maki parameter always larger than one) to describe the pair-breaking effect and the Pauli limiting effect has to be included.

${ }^{75}$ As NMR measurements on $\mathrm{CeFeAsO}_{1-\mathrm{x}} \mathrm{F}_{\mathrm{x}}$ show the appearance of additional peaks in the low temperature region. For the non-superconducting samples with $x=0.00$ and $x=0.07$ this is due to SDW ordering. For the $x=0.07$ sample a spin-density-wave (SDW) ordering temperature at around $20 \mathrm{~K}$ was observed from the NMR data, which was not expected because a SDW should only be observed for $x \leq 0.06$. This as well as the appearance of a broad low temperature NMR peak is consistent with charge inhomogeneities due to a variable fluoride concentration. Additional NMR peaks are 
also seen for the superconducting samples in both field directions and for temperature as high as $100 \mathrm{~K}$, which are due to charge inhomogeneities. Therefore, NMR results show that there are charge inhomogeneities for $x=0.06$ to at least $x=0.20$. Charge inhomogeneities have also been reported in the cuprate superconductors.

The NMR shifts of the main line for $x \geq 0.07$ are dominated by hyperfine coupling to the Ce moments and where the main line can be fitted to a Curie-Weiss temperature dependence with a Curie-Weiss temperature of zero within experimental errors. The additional peak at low temperatures seen in the $x=0.13$ and $x=0.20$ NMR spectra has a negative Curie-Weiss temperature that indicates antiferromagnetic interactions between the $\mathrm{Ce}$ moments. The NMR linewidths are all dominated by dipole interactions with the Ce moments and they display a Curie-Weiss temperature equal to zero within experimental errors for the main peak.

The spin-lattice relaxation can be interpreted with Moriya's theory for weakly itinerant 2D antiferromagnets [49, 190]. This mechanism is strongest in $c \| B_{0}$, because it is a hyperfine interaction, which only has non-zero matrix elements in this direction. Therefore, the spin relaxation is through $2 \mathrm{D}$ spin fluctuations. The spin-spin relaxation can be fitted with a Lorentzian shape over the whole doping range at all temperatures. However, for $\mathrm{CeFeAsO}_{0.93} \mathrm{~F}_{0.07}$ is it necessary to use two components to achieve a satisfying fit in the low temperature region. The second component arises from the spin fluctuations of $\mathrm{Fe}$ and it is very short in comparison to the first component already observed for higher temperatures.

The second group comprises single crystal samples of $\mathrm{BaFe}_{2} \mathrm{As}_{2}, \mathrm{SrFe}_{2} \mathrm{As}_{2}$ and low energy $\mathrm{Ca}$ implanted $\mathrm{SrFe}_{2} \mathrm{As}_{2}$ where their magneto-transport properties were studied. While the former shows no sign of superconductivity, a mixed superconducting and normal state was detected in the later two. A large magnetoresistance is observed in the normal state and it is shown that this is unlikely due to quantum linear magnetoresistance. It was found that the magnetoresistance can be modeled with a three-carrier transport model for the non-superconducting samples and in the high field region for the superconducting ones. An enhanced magnetoresistance is observed in 
the superconducting samples below $T_{\mathrm{C}}$, with a magnetoresistance as high as $1600 \%$ for aged $\mathrm{SrFe}_{2} \mathrm{As}_{2}$. This increase in the MR at low temperatures was interpreted as three-carrier transport in an inhomogeneous state with metallic and superconducting regions and enhanced carrier mobilities. 


\subsection{Iron-copper oxide hybrids}

Polycrystalline samples of the iron-copper hybrid materials $\mathrm{FeSr}_{2} \mathrm{YCu}_{2} \mathrm{O}_{6+\mathrm{y}}$ (Fe1212) and $\mathrm{FeSr}_{2} \mathrm{Y}_{2-\mathrm{x}} \mathrm{Ce}_{\mathrm{x}} \mathrm{Cu}_{2} \mathrm{O}_{10-\mathrm{y}}(\mathrm{Fe} 1222)$ were investigated by electronic transport, magnetic and magnetotransport measurements as a function of doping and impurity substitution. Fe1212 was found to be superconducting with a maximum $T_{\mathrm{C}}$ of $64 \mathrm{~K}$ in the undoped compound. Superconductivity was found to be suppressed by $\mathrm{Fe}$ on the $\mathrm{Cu}(2)$ site causing pair breaking and reducing $T_{\mathrm{C}}^{\max }$ to $64 \mathrm{~K}$ compared to $80 \mathrm{~K}$ for the Fe-free compound. Electron (La) and hole (Ca) doping formed a typical dome-shaped superconducting phase diagram while Zn substitution causes further pair breaking as well as driving more $\mathrm{Fe}$ onto the $\mathrm{Cu}(2)$ site. Further findings include a possible Stoner enhancement observed in the temperature independent part of the susceptibility in all superconducting samples (parent, 0.1 Ca and 0.1 La doping and $\mathrm{x}=0.01-0.03 \mathrm{Zn}$ doping). A decrease in the antiferromagnetic exchange interaction with increasing hole doping was observed and the obtained effective moment is due to a mixture of high and low spin configurations of Fe. There is also a noticeable hysteresis in the magnetization versus applied field curves in all samples with Zn substitution, but it is not clear if this is due to magnetic order or an inhomogeneous distribution in the Zn concentration that results in superconducting regions even for higher Zn concentrations.

No sign of superconductivity was found in Fe1222, but a spin-glass transition was observed at $23 \mathrm{~K}$ due to disordered Fe-O-Fe superexchange interactions or site disorder where there are significant fractions of $\mathrm{Fe}$ and $\mathrm{Cu}$ on both transition metal sites. The conduction mechanism is $3 \mathrm{D}$ variable-rangehopping $(\mathrm{VRH})$ with a departure at low temperatures. A magnetoresistance as high as $-22 \%$ at $8 \mathrm{~T}$ and at low temperatures is observed. The magnetoresistance at and above $5 \mathrm{~K}$ is negative and is interpreted as VRH quantum interference when there is scattering from static or frozen spins. A positive magnetoresistance occurs for temperatures below $5 \mathrm{~K}$ that may be due to a contribution from hops where there is scattering from localized free spins that is not accounted for in the Nguen, Spivak, and Shklovskii (NSS) model. The effect of oxygen reduction was also studied on oxygen loaded and oxygen re- 
duced $\mathrm{FeSr}_{2} \mathrm{Y}_{2-\mathrm{x}} \mathrm{Ce}_{\mathrm{x}} \mathrm{Cu}_{2} \mathrm{O}_{10-\mathrm{y}}$ samples. The oxygen content has no significant effect on the spin-glass temperature, but antiferromagnetic order is observed for the oxygen reduced sample, which is due to an ordering of the $\mathrm{Cu}$ moments in the $\mathrm{CuO}_{2}$ plane. The magnitude of the Curie-Weiss temperature is increased in the oxygen reduced samples, which is a sign of enhanced antiferromagnetic interactions in these samples. The effective moment per formula unit decreases in the oxygen reduced samples, but the effective moment per Fe increases. The resistivity data from the oxygen reduced sample can be modeled in terms of variable-range-hopping and conduction across a small gap in a semiconductor with a bandgap of $160 \mathrm{meV}$. The resistivity from the oxygen saturated samples is more than three orders of magnitude lower, and the resistivity can be modeled with a metallic resistivity combined with an additional term that is proportional to $T^{-3 / 2}$. The origin of this term is not clear but it does show that there is significant disorder. 


\subsection{Outlook}

This section will outline possible further projects, which would add further information and help to develop an even better understanding of the presented results. Further measurements that may include Shubnikov-de Haas measurements on the $\mathrm{SrFe}_{2} \mathrm{As}_{2}$ single crystals used in this thesis, would help us to understand the magnetoresistance better by directly measuring concentration and mobility of all charge carriers. It would also be helpful to do a further doping dependent study on $\mathrm{SrFe}_{2} \mathrm{As}_{2}$ to properly map the Dirac cone.

Nuclear quadrupole resonance (NQR) measurements on $\mathrm{CeFeAsO}_{1-\mathrm{x}} \mathrm{F}_{\mathrm{x}}$ could help to improve the knowledge about the internal field from the SDW ordering in low fluoride content samples. It would also be helpful to perform further NMR measurements on a few more superconducting samples that include more heavily doped samples, to better understand the development of the low temperature peaks and their eventual doping dependence. It would also be interesting to see if similar inhomogeneities occur in the other 1111pnictides. This is to determine if the inhomogeneities seen in the Ce1111 is common across the iron-pnictides.

Heat capacity measurements on the iron-cuprates could help to determine the Stoner enhancement in Fe1212 and help to investigate the low temperature spin glass in Fe1222. Mössbauer spectroscopy and X-ray photoemission spectroscopy could help to clarify the iron valence. Neutron and synchrotron $\mathrm{X}$-ray scattering to estimate the occupancy of the oxygen sites. Additional measurements with better homogenized samples could clarify if there is an underlying magnetic order in the $\mathrm{Zn}$ doped $\mathrm{FeSr}_{2} \mathrm{YCu}_{2} \mathrm{O}_{6+\mathrm{y}}$ samples. NQR measurements might also be helpful to understand more about the internal dynamics and to estimate the electric field gradient. Such samples should be able to remove the contribution from a changed iron environment in comparison to the non $\mathrm{Zn}$ doped samples. It would also be helpful to make samples with an even higher Zn content to completely suppress superconductivity through Zn doping alone and therefore allowing easier access to a possible underlying magnetic order. 


\section{Bibliography}

[1] Bardeen J, Cooper L N and Schrieffer J R (1957) Phys. Rev. 108(5) $1175-1204$

[2] Zhao J, Hunag Q, De La Cruz C, Li S, Lynn J W, Chen Y, Green M A, Chen G F, Li G, Li Z, Luo J L, Wang N L and Dai P (2008) Nature Materials 7 953-959

[3] Zhao J, Huang Q, de la Cruz C, Lynn J W, Lumsden M D, Ren Z A, Yang J, Shen X, Dong X, Zhao Z and Dai P (2008) Phys. Rev. B 78(13) 132504

[4] Zhang Y Z, Ren Z A and Zhao Z X (2009) Superconductor Science and Technology 22065012

[5] Yin Z P, Lebègue S, Han M J, Neal B P, Savrasov S Y and Pickett W E (2008) Phys. Rev. Lett. 101(4) 047001

[6] Yang J, Li Z C, Lu W, Yi W, Shen X L, Ren Z A, Che G C, Dong X L, Sun L L, Zhou F and Zhao Z X (2008) Superconductor Science Technology 21082001

[7] Yamashita H, Yashima M, Mukuda H, Kitaoka Y, Shirage P M and Iyo A (2010) Physica C: Superconductivity 470, S1 S375 - S376

[8] Tanabe Y, Huynh K K, Heguri S, Mu G, Urata T, Xu J, Nouchi R, Mitoma N and Tanigaki K (2011) Phys. Rev. B 84(10) 100508 
[9] Sun L, Dai X, Zhang C, Yi W, Chen G, Wang N, Zheng L, Jiang Z, Wei X, Huang Y, Yang J, Ren Z, Lu W, Dong X, Che G, Wu Q, Ding H, Liu J, Hu T and Zhao Z (2010) Europhysics Letters 9157008

[10] Shiroka T, Lamura G, Sanna S, Prando G, De Renzi R, Tropeano M, Cimberle M R, Martinelli A, Bernini C, Palenzona A, Fittipaldi R, Vecchione A, Carretta P, Siri A S, Ferdeghini C and Putti M (2011) Phys. Rev. B 84(19) 195123

[11] Saha S R, Butch N P, Kirshenbaum K, Paglione J and Zavalij P Y (2009) Phys. Rev. Lett. 103(3) 037005

[12] Prozorov R, Tanatar M, Blomberg E, Prommapan P, Gordon R, Ni N, Budako S and Canfield P (2009) Physica C: Superconductivity 469667 $-673$

[13] Sambale S, Williams G V M, Stephen J and Chong S V (2014) Materials Research Express 1046112

[14] Hata Y, Mihara Y, Mochiku T, Suzuki J I, Kakeya I, Kadowaki K, Kita E and Yasouka H (2004) Physica C 417 17-24

[15] Hu W Z, Dong J, Li G, Li Z, Zheng P, Chen G F, Luo J L and Wang N L (2008) Phys. Rev. Lett. 101(25) 257005

[16] Huang Q, Qiu Y, Bao W, Green M A, Lynn J W, Gasparovic Y C, Wu T, Wu G and Chen X H (2008) Phys. Rev. Lett. 101(25) 257003

[17] Tinkham M (2004) Introduction to superconductivity (Dover Publications Inc.)

[18] Ashcroft N W and Mermin D N (1976) Solid state physics 1st ed (Toronto: Thomson Learning) ISBN 0030839939

[19] Omnes H K (1911) Comm. Phys. Lab. Univ. Leiden

[20] Omnes H K (1911) Comm. Phys. Lab. Univ. Leiden

[21] Omnes H K (1911) Comm. Phys. Lab. Univ. Leiden 
[22] Meissner W and Ochsenfeld R (1933) Naturwissenschaften 21 787-788

[23] Bednorz J and Müller K (1986) Zeitschrift für Physik B Condensed Matter 64 189-193

[24] Cohn R (1973) physicstoday 26

[25] Wu M K, Ashburn J R, Torng C J, Hor P H, Meng R L, Gao L, Huang Z J, Wang Y Q and Chu C W (1987) Phys. Rev. Lett. 58(9) 908-910

[26] Tallon J, Buckley R, Gilberd P, Presland M, Brown I, Bowden M, Christian L and Goguel R (1988) Nature 333

[27] Shilling A, Cantoni M, Guo J and Ott H (1993) Nature 363

[28] Drozdov A, Eremets M and Troyan I (2014) arXiv:1412.0460

[29] Kamihara Y, Watanabe T, Hirano M and Hosono H (2008) Journal of the American Chemical Society 130 3296-3297

[30] Ren Z A, Che G C, Dong X L, Yang J, Lu W, Yi W, Shen X L, Li Z C, Sun L L, Zhou F and Zhao Z X (2008) Europhysics Letters 8317002

[31] Wu G, Xie Y L, Chen H, Zhong M, Liu R H, Shi B C, Li Q J, Wang X F, Wu T, Yan Y J, Ying J J and Chen X H (2009) Journal of Physics: Condensed Matter 21142203

[32] Rotter M, Tegel M, Johrendt D, Schellenberg I, Hermes W and Pöttgen R (2008) Phys. Rev. B 78(2) 020503

[33] Chua C, Chena F, Goocha M, Guloyd A, Lorenza B, Lv B, Sasmala K, Tangd Z, Tapp J and Xuea Y (2009) Physica C: Superconductivity $469326-331$

[34] Parker D R, Pitcher M J, Baker P J, Franke I, Lancaster T, Blundell S J and Clarke S J (2009) Chem. Commun. (16) 2189-2191

[35] Mizuguchi Y, Tomioka F, Tsuda S, Yamaguchi T and Takano Y (2008) Applied Physics Letters 93152505 
[36] Hsu F C, Luo J Y, Yeh K W, Chen T K, Huang T W, Wu P M, Lee Y C, Huang Y L, Chu Y Y, Yan D C and Wu M K (2008) Proceedings of the National Academy of Sciences 105 14262-14264

[37] Ge J F, Liu Z L, Liu C, Gao C L, Qian D, Xue Q K, Liu Y and Jia J F (2014) Nature Materials 14 285-289

[38] Abrikosov A (1957) Journal of Physics and Chemistry of Solids 2 199208

[39] Ginzburg V and Landau L (1950) Zh. Eksp. Teor. Fiz 20

[40] London F and London H (1935) Proceedings of the Royal Society of London A: Mathematical, Physical and Engineering Sciences 149 7188

[41] Zhang F C and Rice T M (1988) Phys. Rev. B 37(7) 3759-3761

[42] Mazin I I, Singh D J, Johannes M D and Du M H (2008) Phys. Rev. Lett. 101(5) 057003

[43] Kuroki K, Onari S, Arita R, Usui H, Tanaka Y, Kontani H and Aoki H (2008) Phys. Rev. Lett. 101(8) 087004

[44] Singh D J and Du M H (2008) Phys. Rev. Lett. 100(23) 237003

[45] Haule K, Shim J H and Kotliar G (2008) Phys. Rev. Lett. 100(22) 226402

[46] Ishida H and Liebsch A (2010) Phys. Rev. B 81(5) 054513

[47] Varma C (2010) Nature 468 184-185

[48] Sachdev S and Keimer B (2011) physicstoday 64

[49] Moriya T (1985) Spin Fluctuations in Itinerant Electron Magnetism (Springer)

[50] Moriya T and Ueda K (2000) Advances in Physics 49 555-606 
[51] Scalapino D (1995) Physics Reports 250 329-365

[52] Yanasea Y, Jujob T, Nomurac T, Ikedad H, Hottae T and Yamadad K (2003) Physics Reports 387 1-149

[53] Kittel C (2005) Introduction to Solid State Physics 8th ed (John Wiley \& Sons, Inc)

[54] Dong J, Zhang H J, Xu G, Li Z, Li G, Hu W Z, Wu D, Chen G F, Dai X, Luo J L, Fang Z and Wang N L (2008) Europhysics Letters 83 27006

[55] De La Cruz C, Huang Q, Lynn J W, Li J, Ratcliff II W, Zarestky J, Mook H, Chen G, Luo J, Wang N and Dai P (2008) Nature 453 899-902

[56] McGuire M A, Hermann R P, Sefat A S, Sales B C, Jin R, Mandrus D, Grandjean F and Long G J (2009) New Journal of Physics 11025011

[57] Qiu Y, Bao W, Huang Q, Yildirim T, Simmons J M, Green M A, Lynn J W, Gasparovic Y C, Li J, Wu T, Wu G and Chen X H (2008) Phys. Rev. Lett. 101(25) 257002

[58] Chen Y, Lynn J W, Li J, Li G, Chen G F, Luo J L, Wang N L, Dai P, dela Cruz C and Mook H A (2008) Phys. Rev. B 78(6) 064515

[59] Tranquada J, Sternlieb B, Axe J, Nakamura Y and Uchida S (1995) Nature 375 561-563

[60] Ma F and Lu Z Y (2008) Phys. Rev. B 78(3) 033111

[61] Orenstein J and Vishwanath A (2010) Nature Physics 6 566-567

[62] Mott N F (1949) Proceedings of the Physical Society. Section A 62416

[63] Anderson P W (1959) Phys. Rev. 115(1) 2-13

[64] Hubbard J (1963) Proceedings of the Royal Society of London A: Mathematical, Physical and Engineering Sciences 276 238-257 
[65] Hubbard J (1964) Proceedings of the Royal Society of London A: Mathematical, Physical and Engineering Sciences 277 237-259

[66] Hubbard J (1964) Proceedings of the Royal Society of London A: Mathematical, Physical and Engineering Sciences 281 401-419

[67] Imada M, Fujimori A and Tokura Y (1998) Rev. Mod. Phys. 70(4) $1039-1263$

[68] Chao K A, Spalek J and Oles A M (1977) Journal of Physics C: Solid State Physics 10 L271

[69] Anderson P W (1987) Science 235 1196-1198

[70] Hirsch J E (1985) Phys. Rev. B 31(7) 4403-4419

[71] Fischer K and Hertz J (1991) Spin Glasses (Cambridge University Press)

[72] Mydosh J (1996) Journal of Magnetism and Magnetic Materials 157 606-610

[73] Mydosh J (1993) Spin Glasses: An Experimental Introduction (Taylor \& Francis)

[74] Mydosh J (1978) Journal of Magnetism and Magnetic Materials 7237 $-248$

[75] Mezard M, Parisi G and Virasoro M (1987) Spin Glass Theory and Beyond (World Scienfitic)

[76] Vogel H (1921) Physikalische Zeitschrift 22645

[77] Fulcher G S (1925) Journal of the American Ceramic Society 8 339-355

[78] Fulcher G S (1925) Journal of the American Ceramic Society 8 789-794

[79] Binder K and Young A P (1986) Rev. of Mod.Phys. 58 801-976

[80] Shtrikman S and Wohlfarth E (1981) Physics Letters A 85467 - 470 
[81] Baym G and Pethick C (1991) Landau Fermi-Liquid Theory: Concepts and Apllications (Wiley)

[82] Pines D and Nozieres P (1989) Theory of Quantum Liquids: Normal Fermi Liquids (Addison Wesley)

[83] Neilson D (1996) Australian Journal of Physics 49 79-102

[84] Mott N F (1969) Philosophical Magazine 19 835-852

[85] Mott N F (1972) Journal of non-crystalline materials 11 1-18

[86] Efros A L and Shklovskii B I (1975) Journal of Physics C: Solid State Physics 83

[87] Thomson W (1856) Proceedings of the Royal Society of London 8 546550

[88] Solin S A, Thio T, Hines D R and Heremans J J (2000) Science 289 $1530-1532$

[89] Hewett T H and Kusmartsev F V (2010) Phys. Rev. B 82(21) 212404

[90] Malozemoff A P (1985) Phys. Rev. B 32(9) 6080-6083

[91] McGuire T and Potter R (1975) IEEE Transactions on Magnetics 11 $1018-1038$

[92] Baibich M N, Broto J M, Fert A, Van Dau F N, Petroff F, Etienne P, Creuzet G, Friederich A and Chazelas J (1988) Phys. Rev. Lett. 61(21) $2472-2475$

[93] Binasch G, Grünberg P, Saurenbach F and Zinn W (1989) Phys. Rev. B 39(7) 4828-4830

[94] Julliere M (1975) Physics Letters A 54225 - 226

[95] Lee P A and Ramakrishnan T V (1985) Rev. Mod. Phys. 57(2) 287-337

[96] Bergmann G (1984) Physics Reports 1071 - 58 
[97] Nguen V L, Spivak B Z and Shklovskii B I (1985) Soviet Physics JETP 621021

[98] Ioffe L and Spivak B (2013) Journal of Experimental and Theoretical Physics 117 551-569

[99] Shklovskii B I and Efros A L (1984) Electronic properties of doped semiconductors (Springer Series in Solid-State Sciences vol 45) (Springer)

[100] Shklovskii B I and Efros A L (1983) Soviet Physics JETP 57470

[101] Shklovskii B I (1983) JETP Letters 3651

[102] Shklovskii B I (1983) Soviet Physics Semiconductors 171311

[103] Khaetskii A V and Shklovskii B I (1983) Soviet Physics JETP 58421

[104] Entin-Wohlman O, Imry Y and Sivan U (1989) Phys. Rev. B 40(12) $8342-8348$

[105] Nguen V L, Spivak B Z and Shklovskii B I (1986) JETP Letters 4348

[106] Shklovskii B I and Spivak B Z (1991) Hopping Transport in Solids (Elsivier)

[107] Shklovskii B I and Spivak B Z (1990) Hopping and Related Phenomena (World Scienfitic)

[108] Medina E, Kardar M, Shapir Y and Wang X (1990) Phys. Rev. Lett. 64(15) 1816-1819

[109] Zhao H, Spivak B, Gelfand M and Feng S (1991) Phys. Rev. B 44(19) 10760-10767

[110] Kim J S, Seiler D G and Tseng W F (1993) Journal of Applied Physics $738324-8335$

[111] Kim J S (1998) Journal of Applied Physics 84 292-300 
[112] Slichter C P (1996) Principles of Magnetic Resonance 3rd ed (Springer Series in Solid State Sciences 1 vol 3) (Springer)

[113] Evetts J (2005) Concise Encylopedia of Magnetic and Superconducting materials (Elsevier)

[114] Harris R K, Becker E D, Menezes S M D, Granger P, Hoffman R E and Zilm K W (2008) Mag. Res. Chem. 46 582-598

[115] Williams G V M, Haines E M and Tallon J L (1998) Phys. Rev. B 57(1) $146-149$

[116] Smith D O (1956) Review of Scientific Instruments 27 261-268

[117] Josephson B (1962) Physics Letters 1251 - 253

[118] Josephson B D (1974) Rev. Mod. Phys. 46(2) 251-254

[119] Shirage P M, Miyazawa K, Kito H, Eisaki H and Iyo A (2008) Phys. Rev. B 78172503

[120] Chen G F, Li Z, Wu D, Li G, Hu W Z, Dong J, Zheng P, Luo J L and Wang N L (2008) Phys. Rev. Lett. 100(24) 247002

[121] Ren Z A, Yang J, Lu W, Yi W, Che G C, Dong X L, Sun L L and Zhao Z X (2008) Materials Research Innovations 12105

[122] Chen X H, Wu T, Wu G, Liu R H, Chen H and Fang D F (2008) Nature 453 761-762

[123] Shirage P M, Miyazawa K, Kihou K, Lee C H, Kito H, Tokiwa K, Tanaka Y, Eisaki H and Iyo A (2010) Europhysics Letters 9257011

[124] Bos J W G, Penny G B S, Rodgers J A, Sokolob D A, Huxley A D and Attfield J P (2008) Chemical Communications 31 3534-3635

[125] Chen G F, Li Z, Dong J, Li G, Hu W Z, Zhang X D, Song X H, Zheng P, Wang N L and Luo J L (2008) Phys. Rev. B 78(22) 224512 
[126] Gurevich A (2014) Annual Review of Condensed Matter Physics 5 3556

[127] Gurevich A (2011) Nature M 10 255-259

[128] Gurevich A (2011) Reports on Progress in Physics 74124501

[129] Prakash J, Singh S, Patnaik S and Ganguli A (2009) Physica C: Superconductivity $46982-85$

[130] Shahbazi M, Wang X L, Shekhar C, Srivastava O N and Dou S X (2010) Superconductor Science and Technology 23105008

[131] Chong S, Mochiji T, Sato S and Kadowaki K (2008) Journal of the Physical Society of Japan 77 27-31

[132] Ahilan K, Ning F L, Imai T, Sefat A S, Jin R, McGuire M A, Sales B C and Mandrus D (2008) Phys. Rev. B 78(10) 100501

[133] Matsuishi A, Hanna T, Muraba Y, Kim S W, Kim J E, Takata M, i Shamoto S, Smith R I and Hosono H (2012) Phys. Rev. B 85014514

[134] Maeter H, Borrero J, Goltz T, Spehling J, Kwadrin A, Kondrat A, Veyrat L, Lang G, Grafe H J, Hess C, Behr G, BÃAEchner B, Luetkens H, Baines C, Amato A, Leps N, Klingeler R, Feyerherm R, Argyriou D and Klauss H H (2012) arXiv:1210.6959v1

[135] Hess C, Kondrat A, Narduzzo A, Hamann-Borrero J E, Klingeler R, Werner J, Behr G and Büchner B (2009) Europhysics Letters 8717005

[136] Aslamazov L and Larkin A (1968) Fiz. Tverd. Tela 101104

[137] Williams G V M and Tallon J L (1996) Physica C: Superconductivity $25841-46$

[138] Blatter G, Feigel'man M V, Geshkenbein V B, Larkin A I and Vinokur V M (1994) Rev. Mod. Phys. 66(4) 1125-1388 
[139] Liu S, Haiyun W and Gang B (2010) Physics Letters A 3743529 3532

[140] Song Y, Kang B, Rhee J S and Kwon Y (2012) European Physics Letter $\mathbf{9 7} 47003$

[141] Palstra T T M, Batlogg B, Schneemeyer L F and Waszczak J V (1988) Phys. Rev. Lett. 61(14) 1662-1665

[142] Lei H, Hu R, Choi E S and Petrovic C (2010) Phys. Rev. B 82(13) 134525

[143] Prando G, Carretta P, De Renzi R, Sanna S, Palenzona A, Putti M and Tropeano M (2011) Phys. Rev. B 83(17) 174514

[144] Maki K (1966) Phys. Rev. 148(1) 362-369

[145] Aswathy P M, Anooja J B, Sarun P M and Syamaprasad U (2010) Superconductor Science and Technology 23073001

[146] Bean C P (1964) Rev. Mod. Phys. 36(1) 31-39

[147] Senatore C, Flúkiger R, Cantoni M, Wu G, Liu R and Chen X (2008) Physical Review B: Condensed Matter and Materials Physics 787

[148] Sunwong P, Higgins J S, Tsui Y, Raine M J and Hampshire D P (2013) Superconductor Science and Technology $\mathbf{2 6} 095006$

[149] Dhallé M, Cuthbert M, Johnston M D, Everett J, Flükiger R, Dou S X, Goldacker W, Beales T and Caplin A D (1997) Superconductor Science and Technology 1021

[150] Bulaevskii L N, Daemen L L, Maley M P and Coulter J Y (1993) Phys. Rev. B 48(18) 13798-13816

[151] Daeumling M, Seuntjens J M and Larbalestier D C (1990) Nature 346 332-335 
[152] Ossandon J G, Thompson J R, Christen D K, Sales B C, Kerchner H R, Thomson J O, Sun Y R, Lay K W and Tkaczyk J E (1992) Phys. Rev. B 45(21) 12534-12547

[153] Ding Y, Sun Y, Zhuang J C, Cui L J, Shi Z X, Sumption M D, Majoros M, Susner M A, Kovacs C J, Li G Z, Collings E W and Ren Z A (2011) Superconductor Science and Technology 24125012

[154] Pramanik A K, Harnagea L, Nacke C, Wolter A U B, Wurmehl S, Kataev V and Büchner B (2011) Phys. Rev. B 83(9) 094502

[155] Sharma S, Vinod K, Sundar C S and Bharathi A (2013) Superconductor Science and Technology 26015009

[156] Salem-Sugui S, Ghivelder L, Alvarenga A D, Cohen L F, Yates K A, Morrison K, Pimentel J L, Luo H, Wang Z and Wen H H (2010) Phys. Rev. B 82(5) 054513

[157] Tallon J L, Williams G V M and Loram J W (2000) Physica C: Superconductivity $3389-17$

[158] Sato T, Souma S, Nakayama K, Terashima K, Sugawara K, Takahashi T, Kamihara Y, Hirano M and Hosono H (2008) Journal of the Physical Society of Japan 773

[159] Nakai Y, Ishida K, Kamihara Y, Hirano M and Hosono H (2008) Journal of the Physical Society of Japan 77073701

[160] Grafe H J, Paar D, Lang G, Curro N J, Behr G, Werner J, HamannBorrero J, Hess C, Leps N, Klingeler R and Büchner B (2008) Phys. Rev. Lett. 101(4) 047003

[161] Liu H Y, Jia X W, Zhang W T, Zhao L, Meng J Q, Liu G D, Dong X L, Wu G, Liu R H, Chen X H, Ren Z A, Yi W, Che G C, Chen G F, Wang N L, Wang G L, Zhou Y, Zhu Y, Wang X Y, Zhao Z X, Xu Z Y, Chen C T and Zhou X J (2008) Chinese Physics Letters 25 3761-3764 
[162] Luo J, Loram J, Cooper J and Tallon J (2000) Physica C: Superconductivity 341, Part 31837 - 1840

[163] Tinkham M (1988) Phys. Rev. Lett. 61(14) 1658-1661

[164] Jo Y, Jaroszynski J, Yamamoto A, Gurevich A, Riggs S, Boebinger G, Larbalestier D, Wen H, Zhigadlo N, Katrych S, Bukowski Z, Karpinski J, Liu R, Chen H, Chen X and Balicas L (2009) Physica C: Superconductivity 469566 - 574

[165] Fang A H, Huang F Q, Xie X M and Jiang M H (2010) Journal of the American Chemical Society 132 3260-3261

[166] Joshi A G, Pillai C, Raj P and Malik S (2001) Solid State Communications 118445 - 448

[167] Werthamer N R, Helfand E and Hohenberg P C (1966) Phys. Rev. 147(1) 295-302

[168] Hunte F, Jaroszynski J, Gurevich A, Larbalestier D C, Jin R, Sefat A S, McGuire M A, Sales B C, Christen D K and Mandrus D (2008) Nature 903-905

[169] Lee H S, Bartkowiak M, Park J H, Lee J Y, Kim J Y, Sung N H, Cho B K, Jung C U, Kim J S and Lee H J (2009) Phys. Rev. B 80(14) 144512

[170] Williams G V M, Tallon J L and Pooke D M (2000) Phys. Rev. B 62(13) 9132-9137

[171] Dulčić A, Paar D, Požek M, Williams G V M, Krämer S, Jung C U, Park M S and Lee S I (2002) Phys. Rev. B 66(1) 014505

[172] Almasan C C, de Andrade M C, Dalichaouch Y, Neumeier J J, Seaman C L, Maple M B, Guertin R P, Kuric M V and Garland J C (1992) Phys. Rev. Lett. 69(26) 3812-3815 
[173] Pribulova Z, Klein T, Kacmarcik J, Marcenat C, Konczykowski M, Bud'ko S L, Tillman M and Canfield P C (2009) Phys. Rev. B 79(2) 020508

[174] Klein T, Braithwaite D, Demuer A, Knafo W, Lapertot G, Marcenat C, Rodière P, Sheikin I, Strobel P, Sulpice A and Toulemonde P (2010) Phys. Rev. B 82(18) 184506

[175] Quebe P, Terbächte L and Jeitschko W (2000) Journal of Alloys and Compounds $30270-74$

[176] Ghoshray A, Pahari B, Majumder M, Ghosh M, Ghoshray K, Bandyopadhyay B, Dasgupta P, Poddar A and Mazumdar C (2009) Phys. Rev. B 79(14) 144512

[177] Grafe H J, Lang G, Hammerath F, Paar D, Manthey K, Koch K, Rosner H, Curro N J, Behr G, Werner J, Leps N, Klingeler R, Klauss H H, Litterst F J and Büchner B (2009) New Journal of Physics 11035002

[178] Jeglič P, Bos J W G, Zorko A, Brunelli M, Koch K, Rosner H, Margadonna S and Arčon D (2009) Phys. Rev. B 79(9) 094515

[179] Zener C (1951) Phys. Rev. 82(3) 403-405

[180] Lang G, Grafe H J, Paar D, Hammerath F, Manthey K, Behr G, Werner J and Büchner B (2010) Phys. Rev. Lett. 104(9) 097001

[181] Kobayashi Y, Satomi E, Chul Lee S and Sato M (2010) Journal of the Physical Society of Japan $\mathbf{7 9} 093709$

[182] Mukuda H, Nitta M, Yashima M, Kitaoka Y, M Shirage P, Eisaki H and Iyo A (2010) Journal of the Physical Society of Japan 79113701

[183] Baek S H, Grafe H J, Harnagea L, Singh S, Wurmehl S and Büchner B (2011) Phys. Rev. B 84(9) 094510

[184] Kitagawa K, Katayama N, Ohgushi K, Yoshida M and Takigawa M (2008) Journal of the Physical Society of Japan 77114709 
[185] Kentaro K, Naoyuki K, Kenya O and Masashi T (2009) Journal of the Physical Society of Japan $\mathbf{7 8} 063706$

[186] Imai T, Ahilan K, Ning F, A McGuire M, S Sefat A, Jin R, C Sales B and Mandrus D (2008) Journal of the Physical Society of Japan 77 47-53

[187] Kitagawa S, Nakai Y, Iye T, Ishida K, Kamihara Y, Hirano M and Hosono H (2010) Phys. Rev. B 81(21) 212502

[188] Prando G, Carretta P, Rigamonti A, Sanna S, Palenzona A, Putti M and Tropeano M (2010) Phys. Rev. B 81(10) 100508

[189] Majumder M, Ghoshray K, Ghoshray A, Pal A and P S Awana V (2012) Journal of the Physical Society of Japan 81054702

[190] Moriya T (1963) Journal of the Physical Society of Japan 18516

[191] Ishigaki A and Moriya T (1998) Journal of the Physical Society of Japan 67 3924-3935

[192] Kondo H (2002) Journal of the Physical Society of Japan 12 3011-15

[193] Rybicki D, Meissner T, Williams G V M, Chong S V, Lux M and Haase J (2013) Journal of Physics: Condensed Matter 25315701

[194] Mukuda H, Terasaki N, Yashima M, Nishimura H, Kitaoka Y and Iyo A (2009) Physica C: Superconductivity 469559 - 565

[195] Kasinathan D, Ormeci A, Koch K, Burkhardt U, Schnelle W, LeitheJasper A and Rosner H (2009) New Journal of Physics 11025023

[196] Ishida S, Liang T, Nakajima M, Kihou K, Lee C H, Iyo A, Eisaki H, Kakeshita T, Kida T, Hagiwara M, Tomioka Y, Ito T and Uchida S (2011) Phys. Rev. B 84(18) 184514

[197] Chong S V, Williams G V M, Kennedy J, Fang F, Tallon J L and Kadowaki K (2013) Europhysics Letters 104 17002-17007 
[198] Richard P, Nakayama K, Sato T, Neupane M, Xu Y M, Bowen J H, Chen G F, Luo J L, Wang N L, Dai X, Fang Z, Ding H and Takahashi T (2010) Phys. Rev. Lett. 104(13) 137001

[199] Sutherland M, Hills D J, Tan B S, Altarawneh M M, Harrison N, Gillett J, O'Farrell E C T, Benseman T M, Kokanovic I, Syers P, Cooper J R and Sebastian S E (2011) Phys. Rev. B 84(18) 180506

[200] Okuda T, Hirata W, Takemori A, Suzuki S, Saijo S, Miyasaka S and Tajima S (2011) Journal of the Physical Society of Japan 80044704

[201] Pinsard-Gaudart L, Bärardan D, Bobroff J and Dragoe N (2008) physica status solidi (RRL) Rapid Research Letters 2 185-187

[202] Abrikosov A A (2000) Europhysics Letters 49789

[203] Meena R S, Singh S K, Pal A, Kumar A, Jha R, Rao K V R, Du Y, Wang X L and Awana V P S (2012) Journal of Applied Physics 111 $07 \mathrm{E} 323$

[204] Bhoi D, Mandal P, Choudhury P, Pandya S and Ganesan V (2011) Applied Physics Letters 98172105

[205] Terashima T, Kurita N, Tomita M, Kihou K, Lee C H, Tomioka Y, Ito T, Iyo A, Eisaki H, Liang T, Nakajima M, Ishida S, Uchida S i, Harima $\mathrm{H}$ and Uji S (2011) Phys. Rev. Lett. 107(17) 176402

[206] Luo H, Wang Z, Yang H, Cheng P, Zhu X and Wen H H (2008) Superconductor Science and Technology 21125014

[207] Wang X F, Wu T, Wu G, Chen H, Xie Y L, Ying J J, Yan Y J, Liu R H and Chen X H (2009) Phys. Rev. Lett. 102(11) 117005

[208] Chong S V, Tallon J L, Fang F, Kennedy J, Kadowaki K and Williams G V M (2011) Europhysics Letters 9437009

[209] Biersack J (1987) Nuclear Instruments and Methods in Physics Research Section B: Beam Interactions with Materials and Atoms 19 to 20, Part $132-39$ 
[210] Yuan H Q, Jiao L, Singleton J, Balakirev F F, Chen G F, Luo J L and Wang N L (2011) Journal of Physics: Conference Series 273012110

[211] Chong S V, Williams G V M, Sambale S, Kennedy J and Kadowaki K (2014) International Journal of Nanotechnology 11 403-11

[212] Engelmann J, Grinenko V, Chekhonin P, Skrotzki W, Efremov D, Oswald S, Iida K, HÃ Ehne R, HÃ Enisch J, Hoffmann M, Kurth F, Schultz L and Holzapfel B (2013) Nature Communications 4

[213] Ishikawa F, Eguchi N, Kodama M, Fujimaki K, Einaga M, Ohmura A, Nakayama A, Mitsuda A and Yamada Y (2009) Phys. Rev. B 79(17) 172506

[214] Kotegawa H, Sugawara H and Tou H (2009) Journal of the Physical Society of Japan 78013709

[215] Colombier E, Bud'ko S L, Ni N and Canfield P C (2009) Phys. Rev. B $79(22) 224518$

[216] Lee H, Park E, Park T, Sidorov V A, Ronning F, Bauer E D and Thompson J D (2009) Phys. Rev. B 80(2) 024519

[217] Huynh K K, Tanabe Y and Tanigaki K (2011) Phys. Rev. Lett. 106(21) 217004

[218] Abrikosov A A (1998) Phys. Rev. B 58(5) 2788-2794

[219] Hamrita A, Azzouz F B, Madani A and Salem M B (2012) Physica C: Superconductivity $\mathbf{4 7 2} 34-38$

[220] Hiramatsu H, Katase T, Kamiya T, Hirano M and Hosono H (2009) Phys. Rev. B 80(5) 052501

[221] Ma F j, Lu Z y and Xiang T (2010) Frontiers of Physics in China 5 $150-160$

[222] Wosik J, Xie L M, Chau R, Samaan A, Wolfe J C, Selvamanickam V and Salama K (1993) Phys. Rev. B 47(14) 8968-8977 
[223] Felner I, Schnmitt D and Barbara B (1997) Physica B 229 153-166

[224] Bernhard C, Tallon J L, Niedermayer C, Blasius T, Golnik A, Brücher E, Kremer R K, Noakes D R, Stronach C E and Ansaldo E J (1999) Phys. Rev. B 59(21) 14099-14107

[225] Sonin E B and Felner I (1998) Phys. Rev. B 57(22) R14000-R14003

[226] Williams G V M and Ryan M (2001) Phys. Rev. B 644515

[227] Williams G V M, Jang L Y and Liu R S (2002) Phys. Rev. B 65 $064508(5)$

[228] Yamaguchi K, Hata Y, Mochiku T and Yasuokaa H (2012) Physica Procedia 27 24-27

[229] Fujii H, Mihara Y, Mochiku T, Hata Y and Kadowaki K (2004) Physica C $41585-93$

[230] Wu M K, Ashburn J R, Higgins C A, Loo B H, Burns D H, Ibrahim A, Rolin T D, Chien F Z and Huang C Y (1988) Phys. Rev. B 37(16) 9765-9768

[231] Xu Y, Suenaga M, Tafto J, Sabatini R L, Moodenbaugh A R and Zolliker P (1989) Phys. Rev. B 39(10) 6667-6680

[232] Tarascon J M, Barboux P, Miceli P F, Greene L H, Hull G W, Eibschutz M and Sunshine S A (1988) Phys. Rev. B 37(13) 7458-7469

[233] Ullmann B, Wördenweber R, Heinemann K and Freyhardt H (1990) Physica C: Superconductivity $\mathbf{1 7 0} 71$ - 79

[234] Pissas M, Kallias G, Moraitakis E, Niarchos D and Simopoulos A (1994) Physica C: Superconductivity $234127-136$

[235] Hata Y, Yamaugchi K, Kinoshita K, Kita E, Mochiku T and Yasuoka H (2014) Physica C: Superconductivity and its Applications 50785 89 
[236] Kirillov D, Collman J P, McDevitt J T, Yee G T, Holcomb M J and Bozovic I (1988) Phys. Rev. B 37(7) 3660-3663

[237] Fainstein A, Pantoja A E, Trodahl H J, McCrone J E, Cooper J R, Gibson G, Barber Z and Tallon J L (2001) Phys. Rev. B 63(14) 144505

[238] Fainstein A, Pregliasco R G, Williams G V M and Trodahl H J (2002) Phys. Rev. B 65 184517(7)

[239] Pringle D J, Tallon J L, Walker B G and Trodahl H J (1999) Phys. Rev. B 59(18) R11679-R11682

[240] Pissas M, Kallias G, Poulakis N, Niarchos D, Simopoulos A and Liarokapis E (1995) Phys. Rev. B 5210610

[241] Fainstein A, Etchegoin P, Trodahl H J and Tallon J L (2000) Phys. Rev. B 61(22) 15468-15473

[242] Williams G and Kramer S (2008) Phys. Rev. B 624132

[243] Shimoyama J, Otzschi K, Hinouchi T and Kishio K (2000) Physica C: Superconductivity 341-348, Part 1563 - 564

[244] Tallon J L, Bernhard C, Williams G V M and Loram J W (1997) Phys. Rev. Lett. 79(26) 5294-5297

[245] Obertelli S, Cooper J and Tallon J (1992) Phys. Rev. B 46(22) 1492814931

[246] Felner I and Asaf U (1998) Superlattices and Microstructures 24 99-104

[247] Felner I and Nowik I (1995) Supercond. Sci. Tech. 8121

[248] Pissas M, Christides C, Kallias G, Niarchos D and Sonntag R (1998) J. Phys Condens Matter 103929

[249] Shumilin A and Kozub V (2012) Phys. Rev. B 85(11) 115203

[250] Vaknin A, Frydman A, Ovadyahu Z and Pollak M (1996) Phys. Rev. B 54(19) 13604-13610 
[251] Sybous A, Kaaouachi A E, Hemine J, Narjis A, Limouny L, Dlimi S, Abdia R and Biskupski G (2012) Journal of Modern Physics 3 521-8

[252] Rosenbaum R, Murphy T, Palm E, Hannahs S and Brandt B (2001) Phys. Rev. B 63(9) 094426

[253] Arushanov K, Lisunov K, Malang U, Kloc C and Bucher E (1997) Phys. Rev. B 56(3) 1005-1008

[254] Abdia R, Kaaouachi A E, Nafidi A, Biskupski G and Hemine J (2009) Solid-State Electronics 53469 - 472

[255] Faran O and Ovadyahu Z (1988) Phys. Rev. B 38(8) 5457-5465

[256] Sladek R (1958) Journal of Physics and Chemistry of Solids 5157 170

[257] Tremblay F, Pepper M, Newbury R, Ritchie D, Peacock D, Frost J, Jones G and Hill G (1989) Phys. Rev. B 40(14) 10052-10055

[258] Tremblay F, Pepper M, Newbury R, Ritchie D, Peacock D, Frost J, Jones G and Hill G (1990) Phys. Rev. B 41(12) 8572-8575

[259] Zhang Y and Sarachik M (1991) Phys. Rev. B 43(9) 7212-7215

[260] Medina E, Kardar M and Rangel R (1996) Phys. Rev. B 53(12) 76637672

[261] Tremblay F, Pepper M, Ritchie D, Peacock D, Frost J and Jones G (1989) Phys. Rev. B 39(11) 8059-8061

[262] Miyatake T, Yamaguchi K, Takata T, Koshizuka N and Tanaka S (1991) Phys. Rev. B 44(18) 10139-10145

[263] Doroshev V, Krivoruchko V, Savosta M, Shestakov A and Tarasenko $\mathrm{T}$ (1996) Journal of Magnetism and Magnetic Materials 157-158 $669 / 670$

[264] Lombardi A, Mali M, Roos J and Brinkmann D (1996) Phys. Rev. B 53(21) 14268-14273 
Appendix A

XRD and SEM figures 


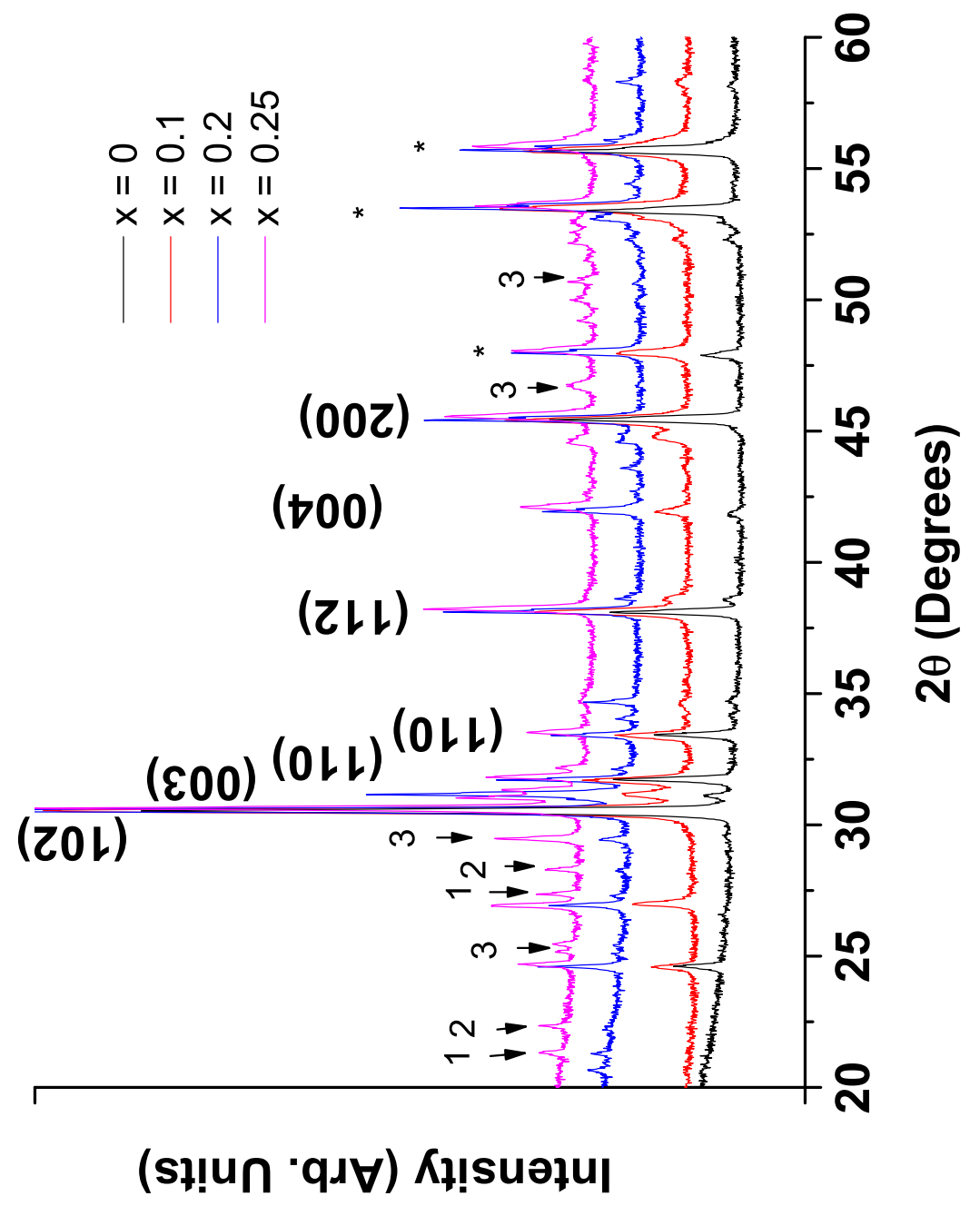

Figure A.1: XRD of $\mathrm{CeFeAsO}_{1-\mathrm{x}} \mathrm{F}_{\mathrm{x}}$, where the expected phase is identified and their crystal planes are shown in brackets. The arrows at lower angles show impurities, which are $1=\mathrm{FeAs}, 2=\mathrm{FeAs}_{2}$ and $3=\mathrm{Ce}_{3} \mathrm{Fe}_{4} \mathrm{O}_{3}$. The peaks at higher angles $\left(^{*}\right)$ are caused by the sample holder. 


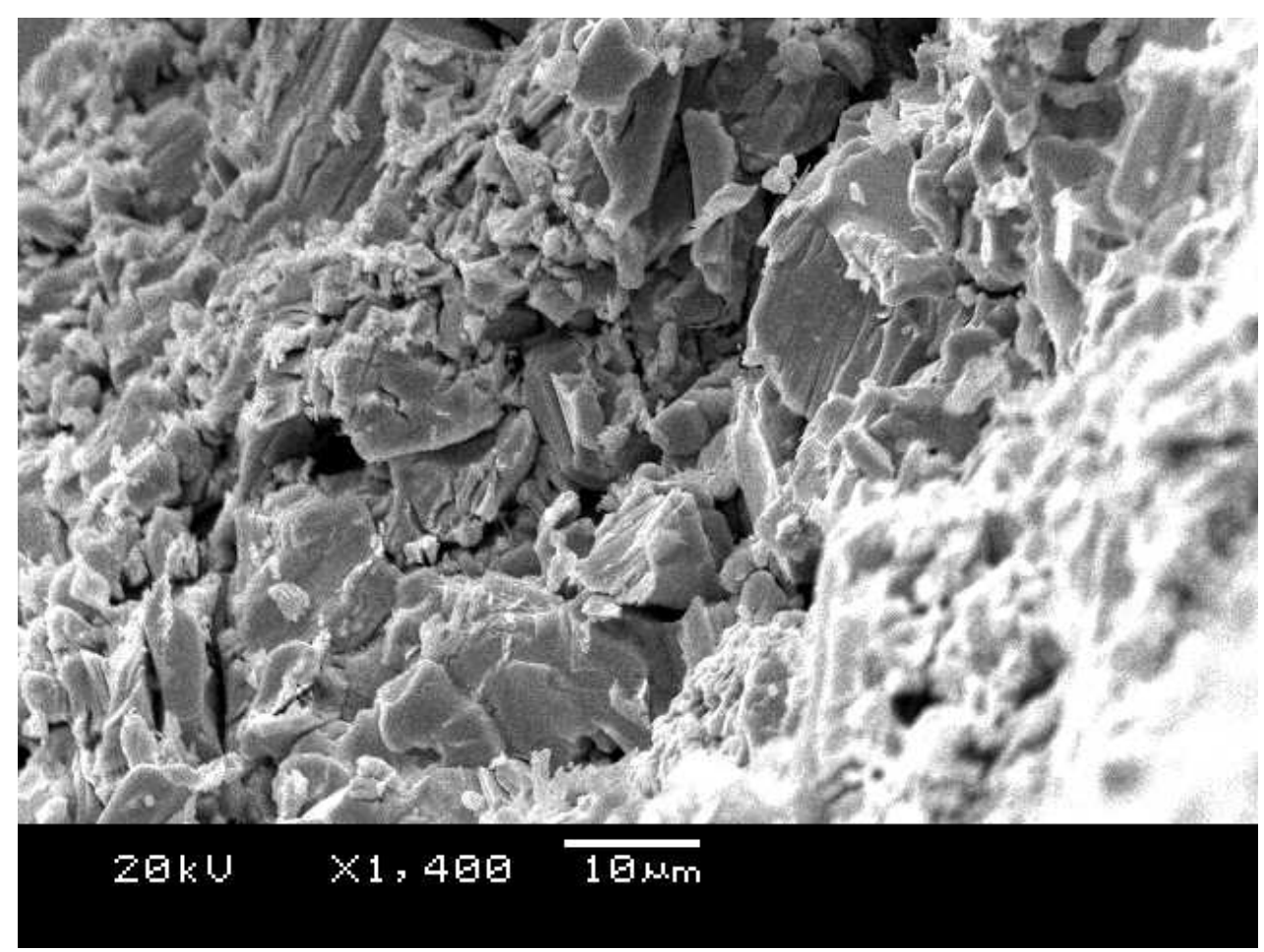

Figure A.2: $\mathrm{SEM}$ of $\mathrm{CeFeAsO}_{0.87} \mathrm{~F}_{0.13}$ 


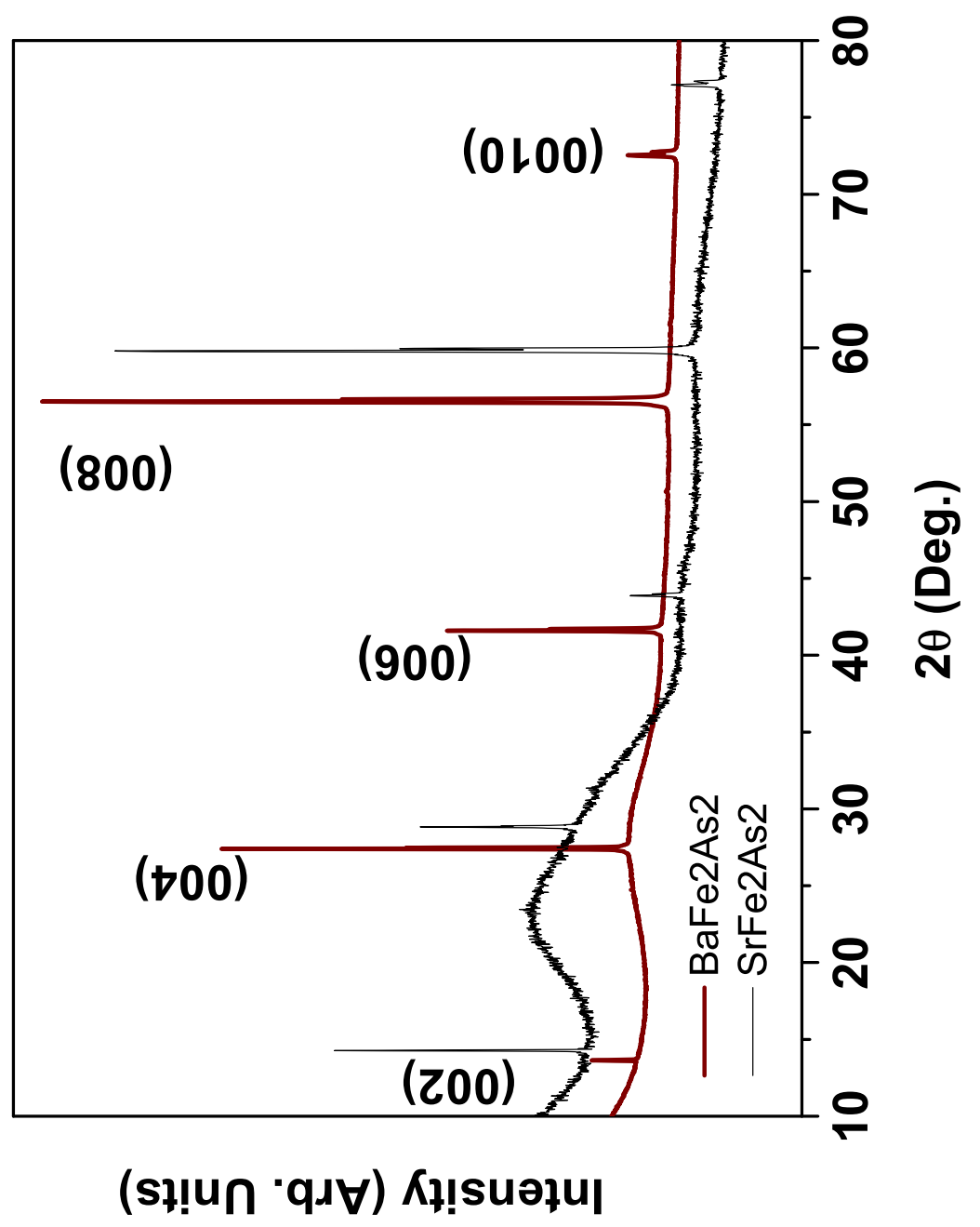

Figure A.3: XRD of $\mathrm{AFe}_{2} \mathrm{As}_{2}(\mathrm{~A}=\mathrm{Ba}, \mathrm{Sr})$. 


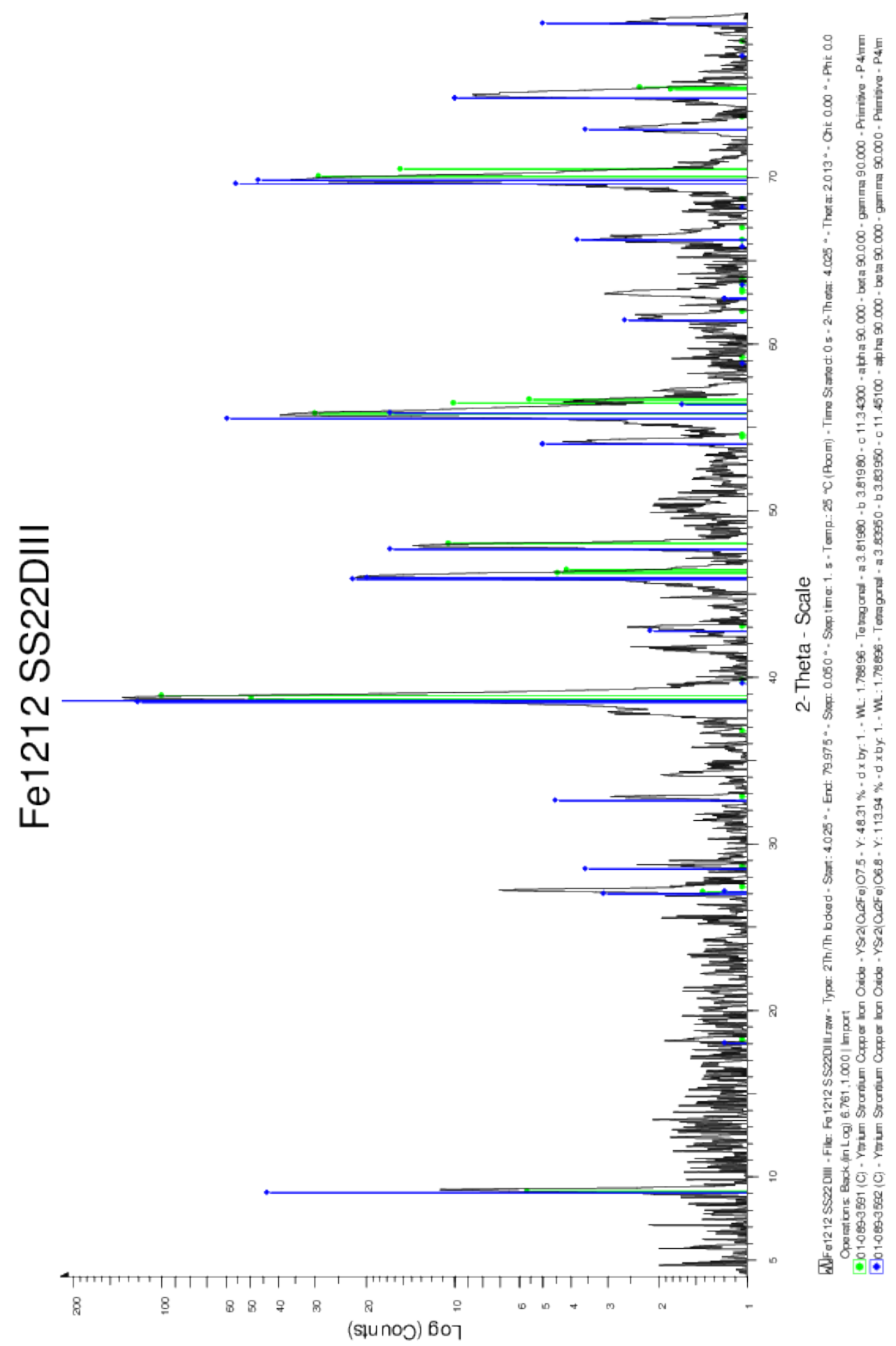

Figure A.4: The measured pattern of pure $\mathrm{FeSr}_{2} \mathrm{YCu}_{2} \mathrm{O}_{6+\mathrm{y}}$ in black and the corresponding theoretical patterns of $\mathrm{FeSr}_{2} \mathrm{YCu}_{2} \mathrm{O}_{7.5}$ in green and $\mathrm{FeSr}_{2} \mathrm{YCu}_{2} \mathrm{O}_{6.8}$ in blue. 


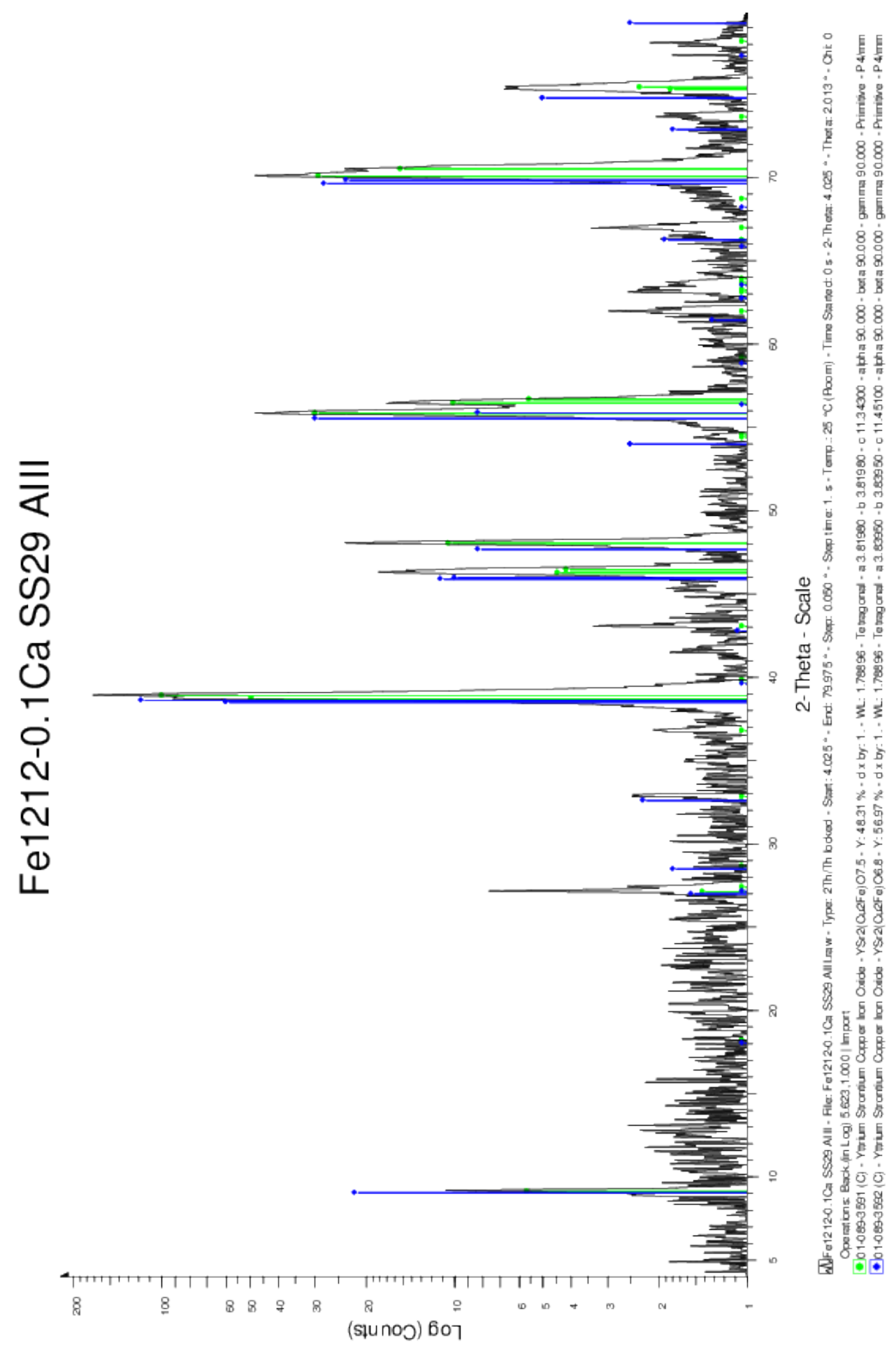

Figure A.5: The measured pattern of $\mathrm{FeSr}_{2} \mathrm{YCu}_{2} \mathrm{O}_{6+\mathrm{y}}$ with $0.1 \mathrm{Ca}$ in black and the corresponding theoretical patterns of $\mathrm{FeSr}_{2} \mathrm{YCu}_{2} \mathrm{O}_{7.5}$ in green and $\mathrm{FeSr}_{2} \mathrm{YCu}_{2} \mathrm{O}_{6.8}$ in blue. 


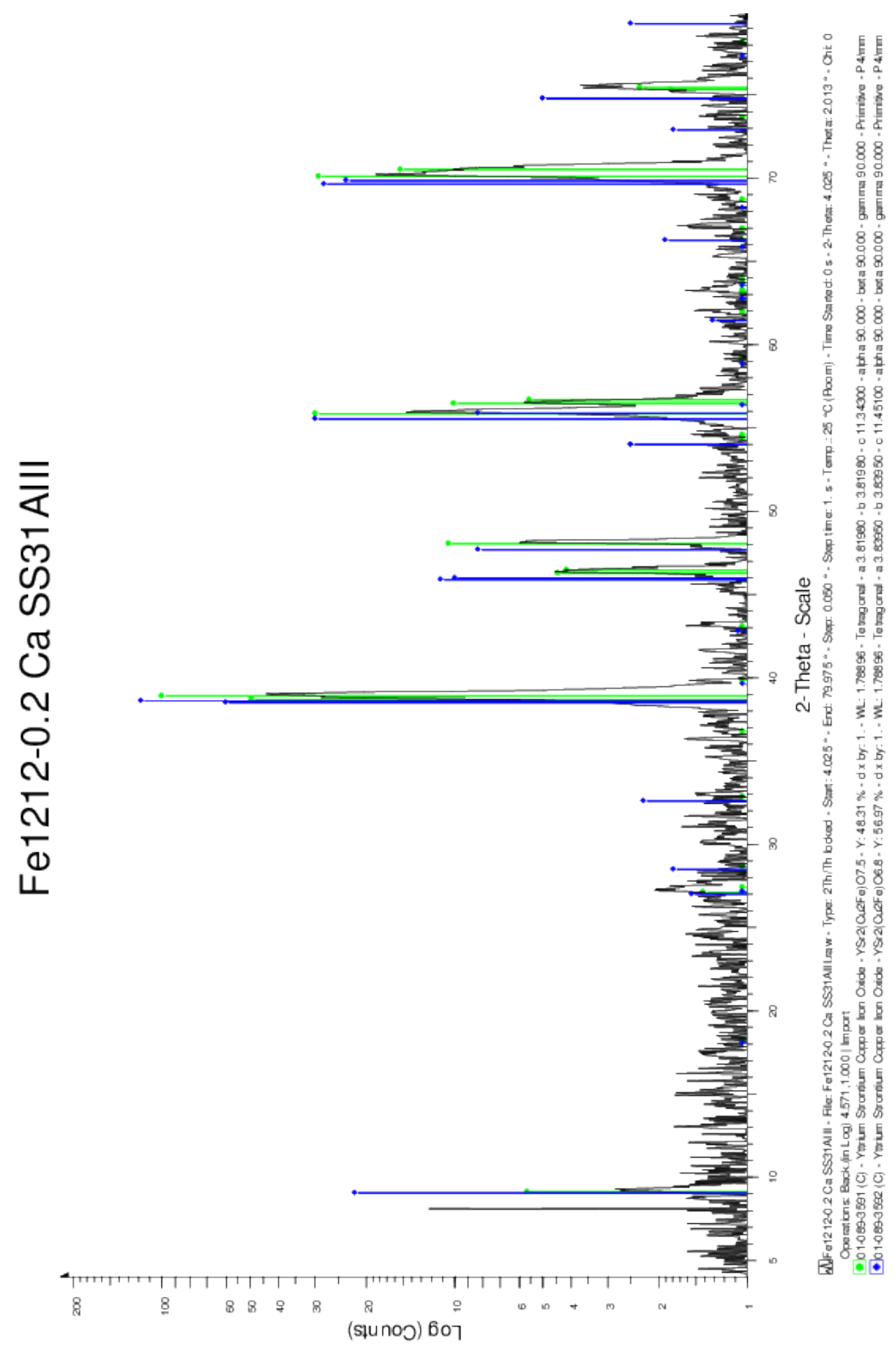

Figure A.6: The measured pattern of $\mathrm{FeSr}_{2} \mathrm{YCu}_{2} \mathrm{O}_{6+\mathrm{y}}$ with $0.2 \mathrm{Ca}$ in black and the corresponding theoretical patterns of $\mathrm{FeSr}_{2} \mathrm{YCu}_{2} \mathrm{O}_{7.5}$ in green and $\mathrm{FeSr}_{2} \mathrm{YCu}_{2} \mathrm{O}_{6.8}$ in blue. 


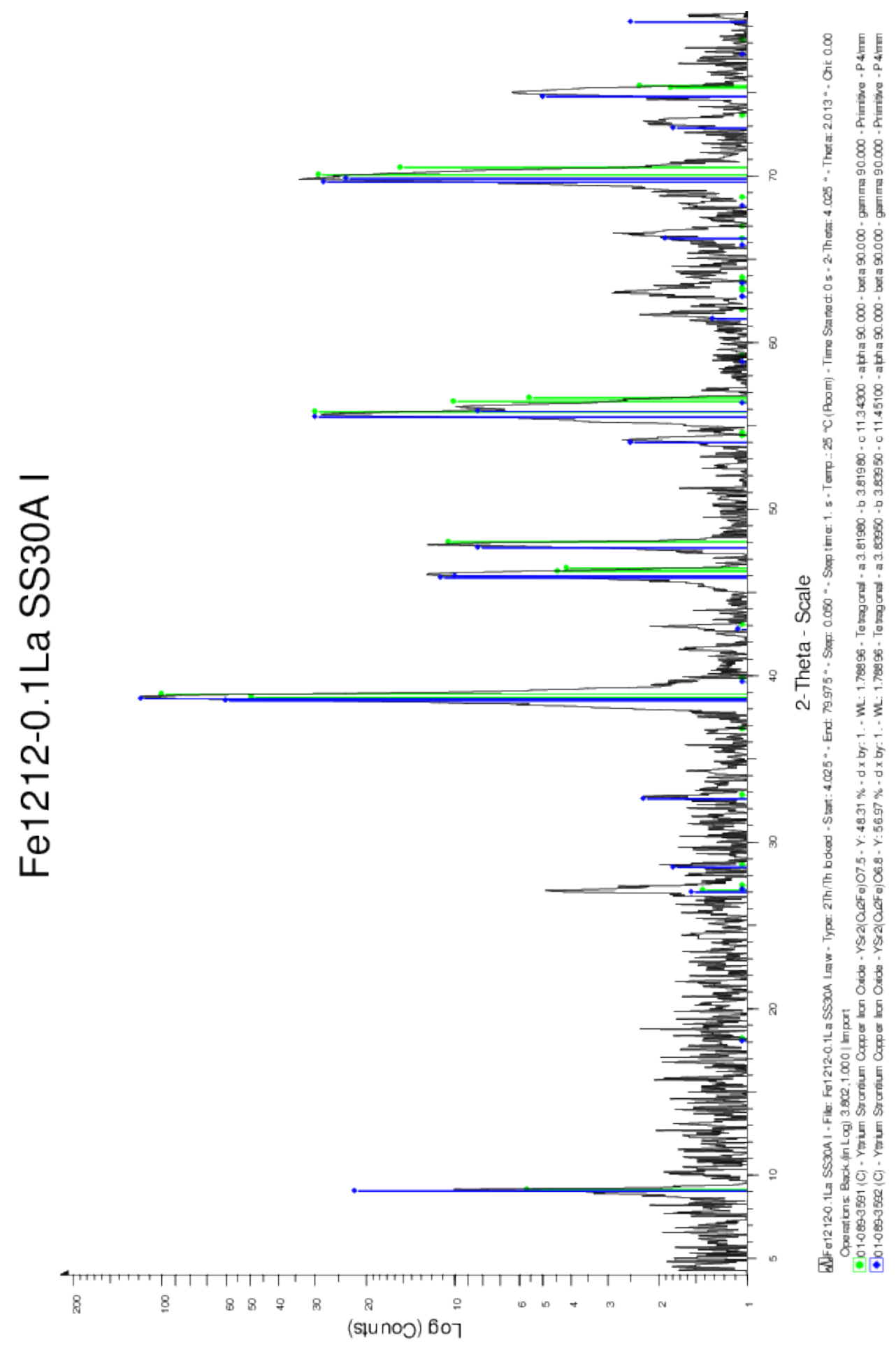

Figure A.7: The measured pattern of $\mathrm{FeSr}_{2} \mathrm{YCu}_{2} \mathrm{O}_{6+\mathrm{y}}$ with $0.1 \mathrm{La}$ in black and the corresponding theoretical patterns of $\mathrm{FeSr}_{2} \mathrm{YCu}_{2} \mathrm{O}_{7.5}$ in green and $\mathrm{FeSr}_{2} \mathrm{YCu}_{2} \mathrm{O}_{6.8}$ in blue. 


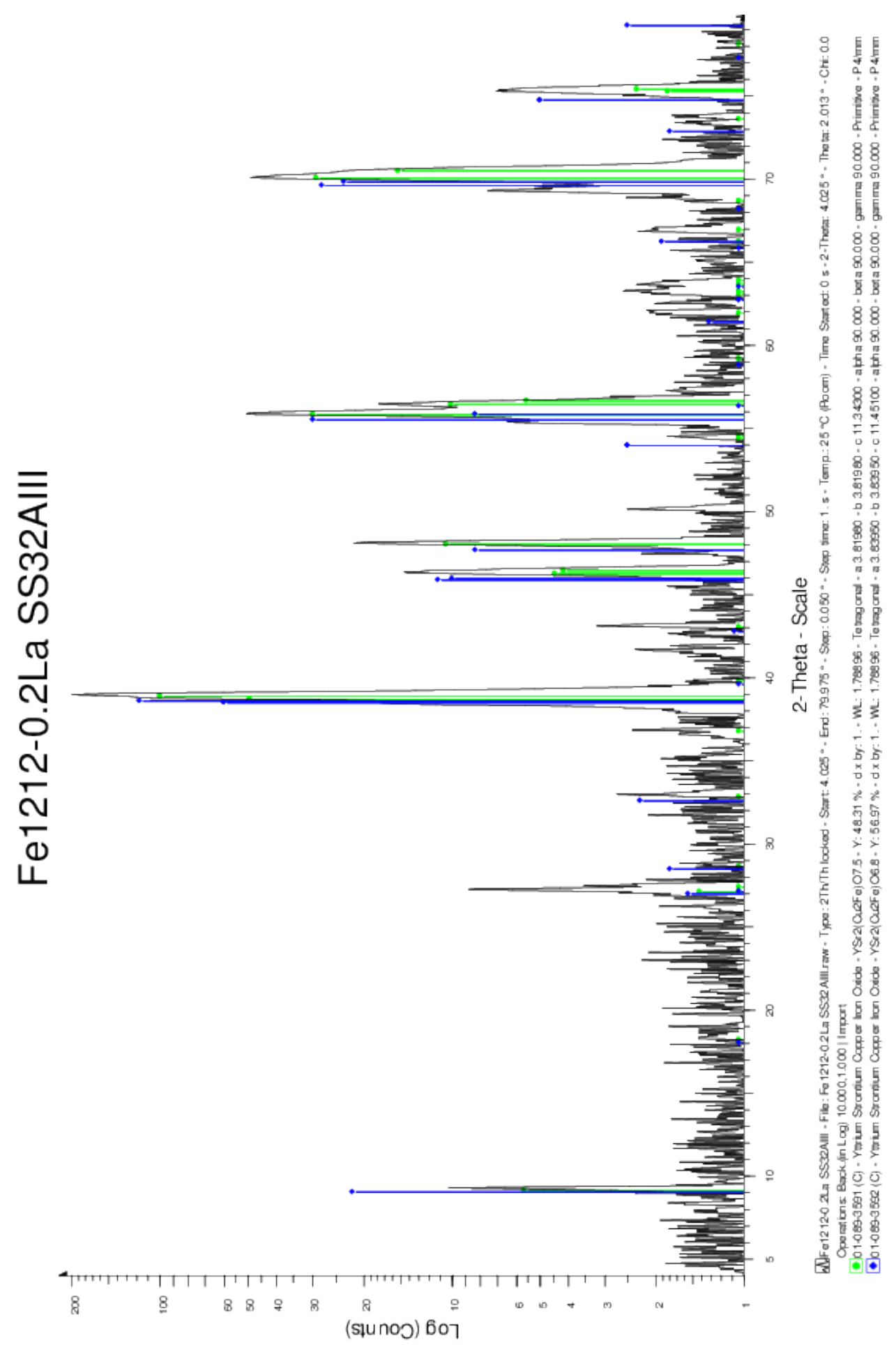

Figure A.8: The measured pattern of $\mathrm{FeSr}_{2} \mathrm{YCu}_{2} \mathrm{O}_{6+\mathrm{y}}$ with $0.2 \mathrm{La}$ in black and the corresponding theoretical patterns of $\mathrm{FeSr}_{2} \mathrm{YCu}_{2} \mathrm{O}_{7.5}$ in green and $\mathrm{FeSr}_{2} \mathrm{YCu}_{2} \mathrm{O}_{6.8}$ in blue. 


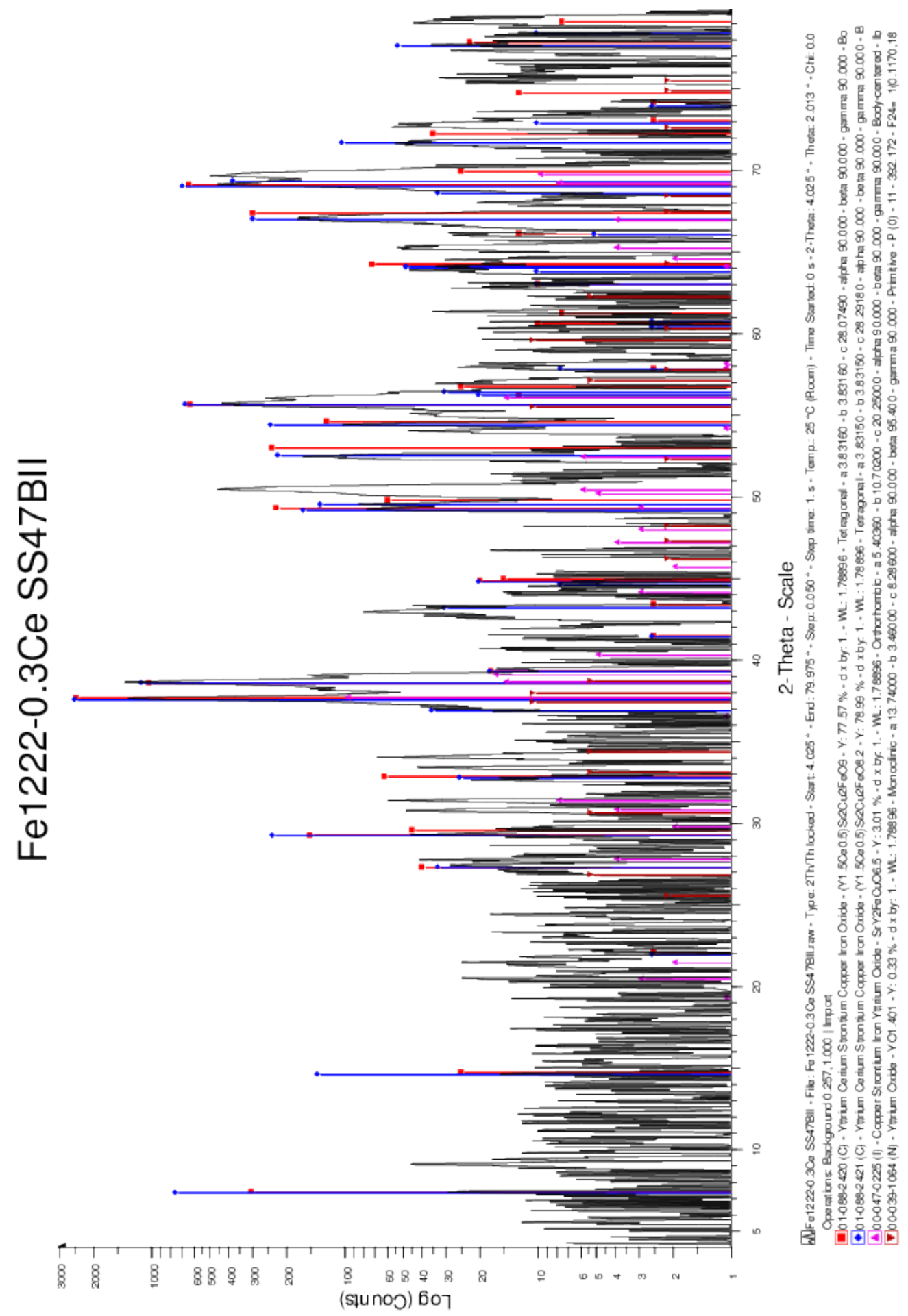

Figure A.9: Measured XRD pattern of $\mathrm{FeSr}_{2} \mathrm{Y}_{1.7} \mathrm{Ce}_{0.3} \mathrm{Cu}_{2} \mathrm{O}_{10-\mathrm{y}}$ in black, the reference patterns of $\mathrm{FeSr}_{2} \mathrm{Y}_{1.5} \mathrm{Ce}_{0.5} \mathrm{Cu}_{2} \mathrm{O}_{9}$ in red and $\mathrm{FeSr}_{2} \mathrm{Y}_{1.5} \mathrm{Ce}_{0.5} \mathrm{Cu}_{2} \mathrm{O}_{8.2}$ in blue as well as two possible impurity phases with $\mathrm{FeSrY}_{2} \mathrm{CuO}_{6.5}$ in pink and $\mathrm{YO}_{1.403}$ in dark red. 


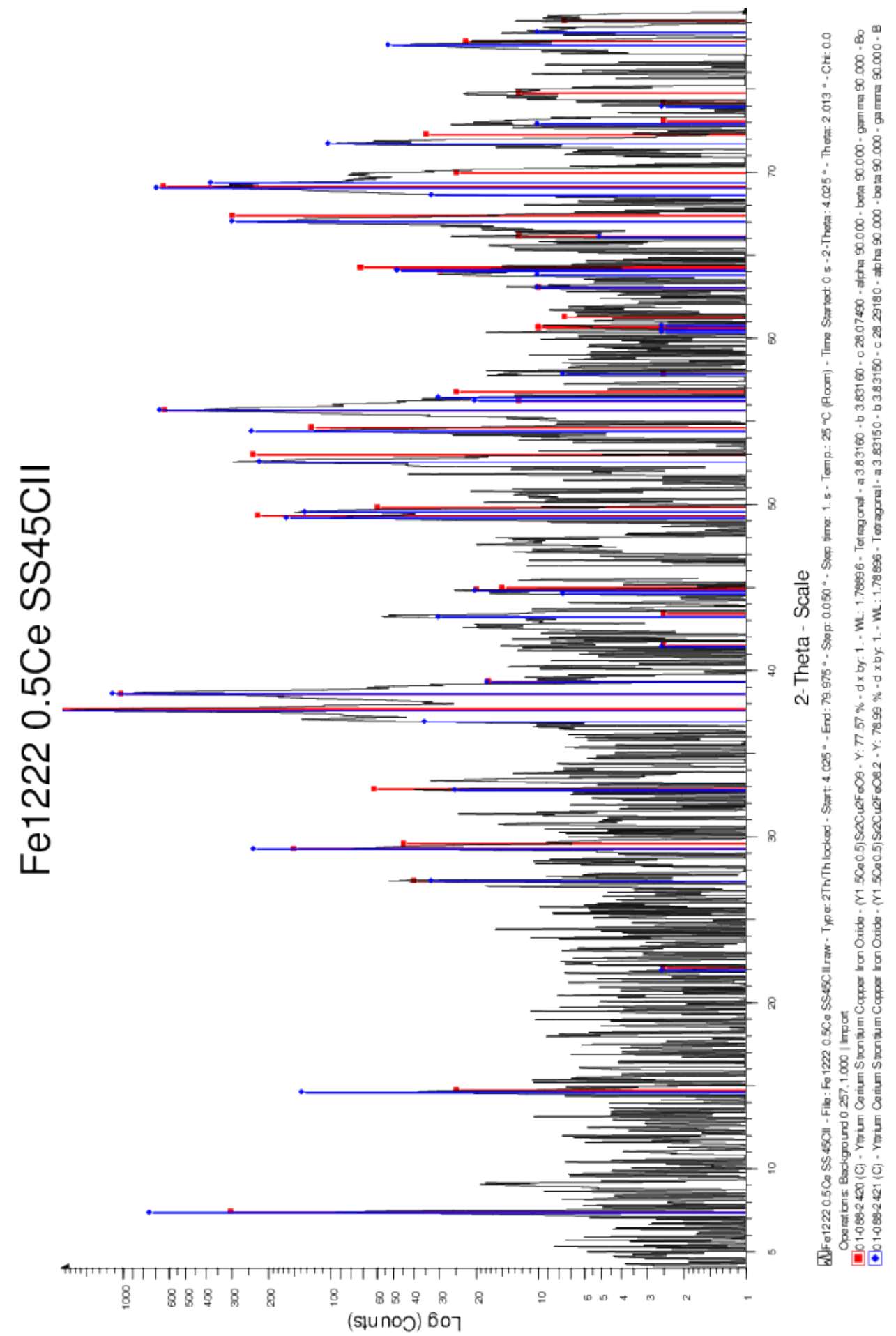

Figure A.10: $\mathrm{FeSr}_{2} \mathrm{Y}_{1.5} \mathrm{Ce}_{0.5} \mathrm{Cu}_{2} \mathrm{O}_{10 \text {-y }}$ in black, the reference patterns of $\mathrm{FeSr}_{2} \mathrm{Y}_{1.5} \mathrm{Ce}_{0.5} \mathrm{Cu}_{2} \mathrm{O}_{9}$ in red and $\mathrm{FeSr}_{2} \mathrm{Y}_{1.5} \mathrm{Ce}_{0.5} \mathrm{Cu}_{2} \mathrm{O}_{8.2}$ in blue 


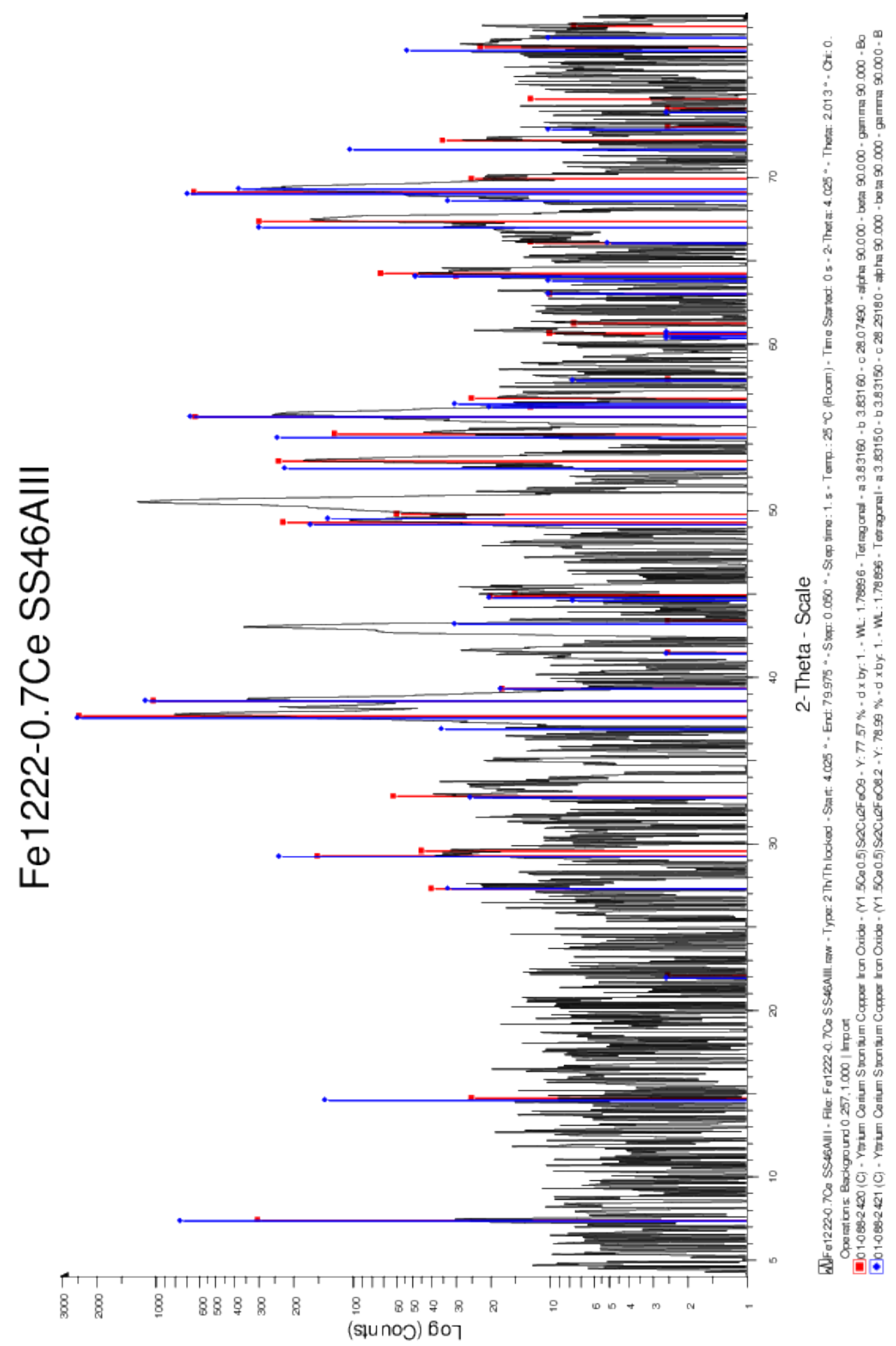

Figure A.11: $\mathrm{FeSr}_{2} \mathrm{Y}_{1.3} \mathrm{Ce}_{0.7} \mathrm{Cu}_{2} \mathrm{O}_{10 \text {-y }}$ in black, the reference patterns of $\mathrm{FeSr}_{2} \mathrm{Y}_{1.5} \mathrm{Ce}_{0.5} \mathrm{Cu}_{2} \mathrm{O}_{9}$ in red and $\mathrm{FeSr}_{2} \mathrm{Y}_{1.5} \mathrm{Ce}_{0.5} \mathrm{Cu}_{2} \mathrm{O}_{8.2}$ in blue 


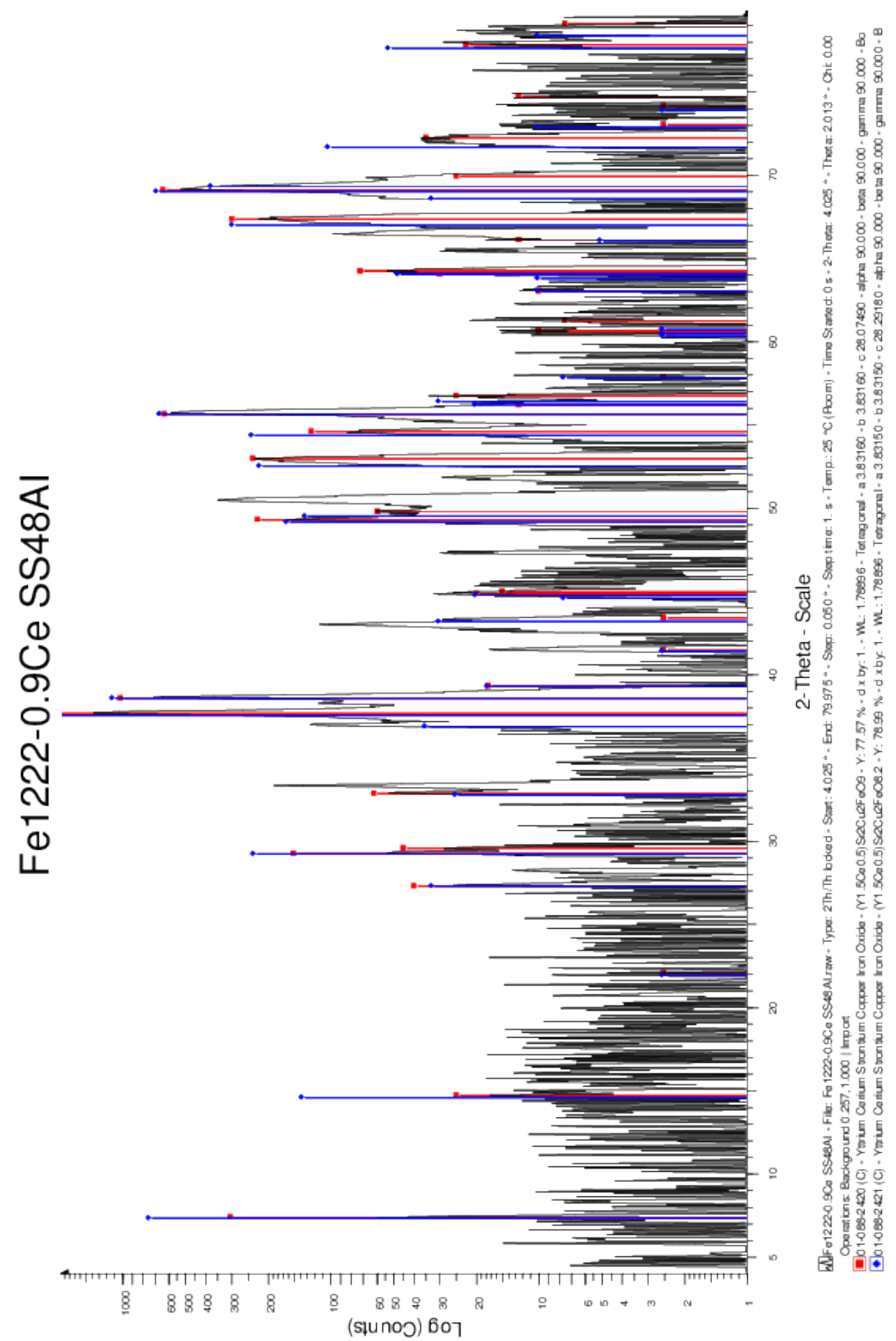

Figure A.12: $\mathrm{FeSr}_{2} \mathrm{Y}_{1.1} \mathrm{Ce}_{0.9} \mathrm{Cu}_{2} \mathrm{O}_{10 \text {-y }}$ in black, the reference patterns of $\mathrm{FeSr}_{2} \mathrm{Y}_{1.5} \mathrm{Ce}_{0.5} \mathrm{Cu}_{2} \mathrm{O}_{9}$ in red and $\mathrm{FeSr}_{2} \mathrm{Y}_{1.5} \mathrm{Ce}_{0.5} \mathrm{Cu}_{2} \mathrm{O}_{8.2}$ in blue 